\title{
Asymmetric Organocatalytic Thio-Diels-Alder Reactions via Trienamine Catalysis
}

Hao Jiang, David Cruz Cruz, Yang Li, Vibeke Henriette Lauridsen and Karl Anker Jørgensen*

\author{
$\left[{ }^{*}\right]$ Center for Catalysis \\ Department of Chemistry, Aarhus University \\ DK-8000 Aarhus C, Denmark \\ Fax (45) 8919 6199, e-mail: kaj@chem.au.dk
}

\section{Contents}

1.

General methods

2.

Starting materials

S3

3. Procedures and products

S5

4. X-Ray structures

5. Computational data

S13

6.

${ }^{1} \mathrm{H}$ and ${ }^{13} \mathrm{C}$ NMR spectra

S97

7.

UPC $^{2}$ traces

S152 


\section{GENERAL METHODS}

NMR spectra were acquired on a Varian AS 400 spectrometer, running at $400 \mathrm{MHz}$ for ${ }^{1} \mathrm{H}$ and $100 \mathrm{MHz}$ for ${ }^{13} \mathrm{C}$, respectively. Chemical shifts $(\delta)$ are reported in ppm relative to residual solvent signals $\left(\mathrm{CHCl}_{3}, 7.26\right.$ ppm for ${ }^{1} \mathrm{H} N M R, \mathrm{CDCl}_{3}, 77.0 \mathrm{ppm}$ for $\left.{ }^{13} \mathrm{C} \mathrm{NMR}\right)$. The following abbreviations are used to indicate the multiplicity in NMR spectra: s, singlet; $d$, doublet; $t$, triplet; q, quartet; hept, heptet; m, multiplet; br, broad resonance. ${ }^{13} \mathrm{C}$ NMR spectra were acquired on a broad band decoupled mode. For characterization of diastereomeric mixtures, *denotes minor diastereoisomer, ${ }^{+}$denotes overlap of signals from both diastereoisomers, while the major diastereomer is characterized without further denotations. The number of protons/carbons given in the parenthesis is the sum over both diastereomers. For compounds with diastereomeric ratios above 6:1, only the major diastereomer is characterized. Mass spectra were recorded on a Bruker MicroTOF-Q High-Performance LC-MS system. Analytical thin layer chromatography (TLC) was performed using pre-coated aluminium-backed plates (Merck Kieselgel 60 F254) and visualized by ultraviolet irradiation, $\mathrm{KMnO}_{4}$ or $p$-anisaldehyde dip. Optical rotations were measured on a Perkin-Elmer 241 polarimeter. The enantiomeric excess (ee) of the products was either determined by: i) Ultraperformance Convergence Chromatography (UPC ${ }^{2}$ ) using Daicel Chiralpak IA-3, IB-3, IC-3 and ID-3 columns as chiral stationary phases; or by ii) chiral stationary phase HPLC on a Daicel Chiralpak IC-5 column. Unless otherwise noted, analytical grade solvents and commercially available reagents were used without further purification. For flash chromatography (FC) silica gel $\left(\mathrm{SiO}_{2} 60,230-400\right.$ mesh, Fluka) was used. Racemic samples were prepared using a mixture of enantiomers of 3 (20 mol\%) in combination with benzoic acid (20 mol\%) in $\mathrm{CHCl}_{3}$. 


\section{STARTING MATERIALS}

Dienals 1a-d,f were prepared from the corresponding alkenyl bromides according to the procedure reported by Fabrizi et al. ${ }^{1}$ Aldehyde $\mathbf{1 e}, \mathbf{h}^{2}$ and dithioester $\mathbf{2} \mathbf{b}^{3}$ were prepared following literature procedures.

\section{General procedure for the preparation of dithioesters $2 a, c-f$}<smiles>[R]C(=O)CCC</smiles>

$\underset{\text { 1) } \mathrm{S}, \mathrm{Et}_{3} \mathrm{~N}, \mathrm{MeCN}, \mathrm{rt}}{\mathrm{R} I}$<smiles>[R]C(=O)C([R])=S</smiles>

To a stirred solution of sulphur ( 30 mmol, 3 equiv.) and $\mathrm{Et}_{3} \mathrm{~N}$ ( $30 \mathrm{mmol}, 3$ equiv.) in $15 \mathrm{~mL}$ MeCN was added halogen compound ( $10 \mathrm{mmol}, 1$ equiv.). After $3 \mathrm{~h}$ of stirring at $\mathrm{rt}$, the alkyl iodide ( $30 \mathrm{mmol}, 3$ equiv.) was added and the mixture was allowed to stir for an additional $15 \mathrm{~h}$. The formed salts were then removed by filtration and the collected solids were washed with small amounts of $\mathrm{MeCN}$. The organic solution were diluted with water and extracted three times with $\mathrm{CH}_{2} \mathrm{Cl}_{2}$. The combined organic layers were washed with a saturated solution of $\mathrm{NaHCO}_{3}$ and $0.5 \mathrm{M} \mathrm{Na}_{2} \mathrm{~S}_{2} \mathrm{O}_{3}$ and dried over $\mathrm{Na}_{2} \mathrm{SO}_{4}$. After filtration of the solids and removal of excess solvents, the crude product was subjected to purification by FC on silica gel to afford the pure dithioesters.<smiles>CCSC(=S)C(=O)OCc1ccccc1</smiles><smiles>CC(=S)C(=O)OCc1ccccc1</smiles><smiles>O=C(OCc1ccccc1)C(=S)c1ccccc1</smiles>

2d<smiles>CCSC(=S)C(=O)OC(C)C</smiles>

$2 e$

${ }^{1} \mathrm{H}$ NMR (400 MHz, CDCl $): \delta$ ppm 7.49-7.30 (m, 5H), $5.34(\mathrm{~s}, 2 \mathrm{H}), 3.25(\mathrm{q}, J=7.5 \mathrm{~Hz}, 2 \mathrm{H})$,

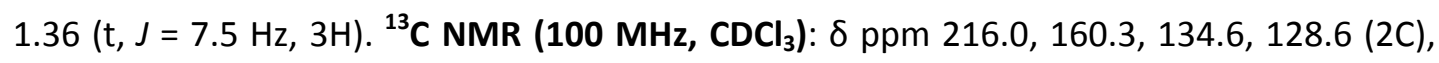
128.5, $128.2(2 \mathrm{C}), 68.6,30.3,11.5$. HRMS (ESI+) $\mathrm{m} / \mathrm{z}$ calcd. for $\mathrm{C}_{11} \mathrm{H}_{12} \mathrm{~S}_{2} \mathrm{O}_{2}[\mathrm{M}+\mathrm{Na}]^{+}: 263.0171$; found: 263.0173 .

${ }^{1} \mathrm{H}$ NMR (400 MHz, $\mathrm{CDCl}_{3}$ ): $\delta \mathrm{ppm} 7.46-7.33(\mathrm{~m}, 5 \mathrm{H}), 5.35(\mathrm{~s}, 2 \mathrm{H}), 2.68(\mathrm{~s}, 3 \mathrm{H}) .{ }^{13} \mathrm{C}$ NMR (100 MHz, $\mathrm{CDCl}_{3}$ ): $\delta$ ppm 216.5, 160.0, 134.5, 128.6 (2C), 128.5, 128.2 (2C), 68.6, 19.5. HRMS (ESI+) $\mathrm{m} / z$ calcd. for $\mathrm{C}_{10} \mathrm{H}_{10} \mathrm{~S}_{2} \mathrm{O}_{2}[\mathrm{M}+\mathrm{Na}]^{+}: 249.0014$; found: 249.0015 .

${ }^{1} \mathrm{H}$ NMR (400 MHz, $\left.\mathrm{CDCl}_{3}\right): \delta \mathrm{ppm} 7.41-7.23(\mathrm{~m}, 10 \mathrm{H}), 5.28(\mathrm{~s}, 2 \mathrm{H}), 4.37(\mathrm{~s}, 2 \mathrm{H}) .{ }^{13} \mathrm{C} \mathrm{NMR}$ (100 MHz, $\mathrm{CDCl}_{3}$ ): $\delta$ ppm 214.9, 159.8, 134.4, 133.5, 129.1 (2C), 128.7 (2C), 128.5 (2C), $128.5,128.2(2 \mathrm{C}), 127.9,68.7,41.0$. HRMS (ESI+) $\mathrm{m} / \mathrm{z}$ calcd. for $\mathrm{C}_{16} \mathrm{H}_{14} \mathrm{~S}_{2} \mathrm{O}_{2}[\mathrm{M}+\mathrm{Na}]^{+}$: 325.0327; found: 325.0328 .

${ }^{1} \mathrm{H}$ NMR $\left(400 \mathrm{MHz}, \mathrm{CDCl}_{3}\right): \delta \mathrm{ppm} 5.22-5.11(\mathrm{~m}, 1 \mathrm{H}), 3.24(\mathrm{q}, J=7.4 \mathrm{~Hz}, 2 \mathrm{H}), 1.37-1.35(\mathrm{~m}$, 9H). ${ }^{13} \mathrm{C} \mathrm{NMR}\left(100 \mathrm{MHz}, \mathrm{CDCl}_{3}\right): \delta$ ppm 217.4, 160.3, 71.4, 30.2, 21.4 (2C), 11.5. HRMS (ESI+) $\mathrm{m} / \mathrm{z}$ calcd. for $\mathrm{C}_{7} \mathrm{H}_{12} \mathrm{~S}_{2} \mathrm{O}_{2}[\mathrm{M}+\mathrm{Na}]^{+}: 215.0171$; found: 215.0171 .

${ }^{1}$ (a) Battistuzzi, G.; Cacchi, S.; Fabrizi, G. Org. Lett. 2003, 5, 777. All spectroscopic data matched those previously reported: (b) Jia, Z.-J.; Zhou, Q.; Zhou, Q.-Q.; Chen, P.-Q.; Chen, Y.-C. Angew. Chem. Int. Ed. 2011, $50,8638$.

${ }^{2}$ For 1e, see (a) Liu, Y.-K.; Nappi, M.; Arceo, E.; Vera, S.; Melchiorre, P. J. Am. Chem. Soc. 2011, 133, 15212. For 1h, see (b) Halskov, K. S.; Johansen, T. K.; Davis, R. L.; Steurer, M.; Jensen, F.; Jørgensen, K. A. J. Am. Chem. Soc. 2012, 134, 12943.

${ }^{3}$ Thiel, W.; Mayer, R. J. Prakt. Chem. 1989, 331, 243. 


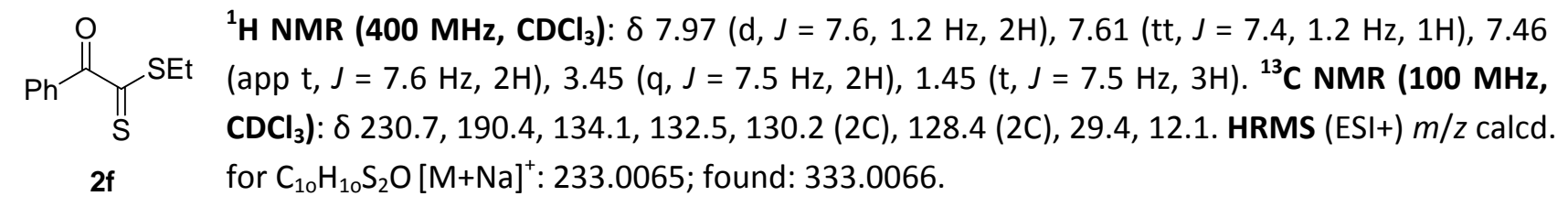




\section{PROCEDURES AND PRODUCTS}

\section{General Procedure A (the linear trienamine pathway)}

A mixture of the aldehyde $1\left(0.1 \mathrm{mmol}, 1\right.$ equiv), $\mathrm{PhCO}_{2} \mathrm{H}(0.02 \mathrm{mmol}, 0.2$ equiv) and catalyst 3 in the given amounts (see the specific substrates in the manuscript) was dissolved in $0.5 \mathrm{~mL} \mathrm{of} \mathrm{CHCl}_{3}$ and brought to the appropriate temperature. Then, the dithioester was added $(0.12 \mathrm{mmol}, 1.2$ equiv) and the mixture was stirred for the time indicated. When the reaction was completed, the mixture was directly purified by FC on silica gel to afford the corresponding cycloadduct.

\section{General procedure B (reduction/NIS-mediated cyclization sequence)}

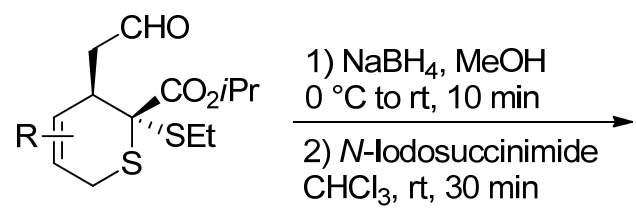

4

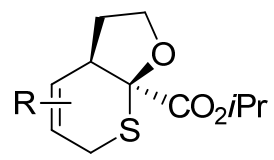

5

To a solution of the aldehyde ( $0.35 \mathrm{mmol}, 1$ equiv) in $\mathrm{MeOH}(3 \mathrm{~mL})$ was added $\mathrm{NaBH}_{4}(0.52 \mathrm{mmol}, 1.5$ equiv) at $0{ }^{\circ} \mathrm{C}$. After stirring for $5 \mathrm{~min}$ the reaction was allowed to reach $\mathrm{rt}$ and stirred for further $10 \mathrm{~min}$. A saturated solution of aq. $\mathrm{NH}_{4} \mathrm{Cl}$ was then added and the resulting solution was extracted three times with $\mathrm{CH}_{2} \mathrm{Cl}_{2}$. The combined organic layers were dried over $\mathrm{Na}_{2} \mathrm{SO}_{4}$ and concentrated in vacuo. The crude alcohol intermediate was re-dissolved in $\mathrm{CHCl}_{3}(1.5 \mathrm{~mL})$ and NIS $(0.42 \mathrm{mmol}, 1.2$ equiv) was added in one portion. Once the reaction was complete ( $30 \mathrm{~min}$ ), the mixture was directly purified by FC on silica gel to afford the pure products.<smiles>CCOC1(OBr)SCC(C)=CC1CC=O</smiles>

$4 a$

Following the general procedure $\mathrm{A}\left(5 \mathrm{~mol} \% 3,4{ }^{\circ} \mathrm{C}, 72 \mathrm{~h}\right.$ ), cycloadduct $4 \mathrm{a}$ was obtained after FC on silica gel (pentane/EtOAc 9:1) in $87 \%$ yield and $94: 6 \mathrm{dr}$ as a pale yellow oil. $[\boldsymbol{\alpha}]_{\mathbf{D}}^{\mathbf{2 0}}=-207.9\left(c 0.3, \mathrm{CH}_{2} \mathrm{Cl}_{2}\right) .{ }^{1} \mathbf{H}$ NMR $\left(400 \mathrm{MHz}, \mathrm{CDCl}_{3}\right): \delta \mathrm{ppm} 9.60(\mathrm{t}, J=1.6 \mathrm{~Hz}, 1 \mathrm{H})$, 7.40-7.32 (m, 5H), $5.54(\mathrm{~d}, J=6.1 \mathrm{~Hz}, 1 \mathrm{H}), 5.25(\mathrm{~d}, J=12.1 \mathrm{~Hz}, 1 \mathrm{H}), 5.19(\mathrm{~d}, J=12.1 \mathrm{~Hz}$, $1 \mathrm{H}), 3.30(\mathrm{~d}, J=17.9 \mathrm{~Hz}, 1 \mathrm{H}), 3.23-3.16(\mathrm{~m}, 1 \mathrm{H}), 2.76-2.68(\mathrm{~m}, 2 \mathrm{H}), 2.66(\mathrm{dq}, J=11.8,7.4$ $\mathrm{Hz}, 1 \mathrm{H}), 2.46(\mathrm{dq}, J=11.8,7.4 \mathrm{~Hz}, 1 \mathrm{H}) 2.33(\mathrm{dd}, J=17.5,3.4 \mathrm{~Hz}, 1 \mathrm{H}), 1.72(\mathrm{~s}, 3 \mathrm{H}), 1.15(\mathrm{t}, J$ $=7.5 \mathrm{~Hz}, 3 \mathrm{H}) .{ }^{13} \mathrm{C}$ NMR (100 MHz, $\left.\mathrm{CDCl}_{3}\right): \delta \mathrm{ppm} 199.7,170.0,134.9,130.5,128.6$ (3C), $128.6(2 \mathrm{C}), 122.6$, 67.7, 62.7, 48.9, 36.9, 29.4, 25.1, 23.8, 13.6. HRMS (ESI+) $\mathrm{m} / z$ calcd. for $\mathrm{C}_{18} \mathrm{H}_{22} \mathrm{~S}_{2} \mathrm{O}_{3}[\mathrm{M}+\mathrm{Na}]^{+}: 373.0903$; found: 373.0907 . $\mathrm{UPC}^{2}$ : IC-3, $\mathrm{CO}_{2} / \mathrm{MeCN} 80: 20,3.0 \mathrm{~mL} \cdot \mathrm{min}^{-1}, 40^{\circ} \mathrm{C}, 120$ bar; $\mathrm{t}_{\text {major }}=4.7 \mathrm{~min} ; \mathrm{t}_{\text {minor }}=7.7 \mathrm{~min}$ (92\% ee).

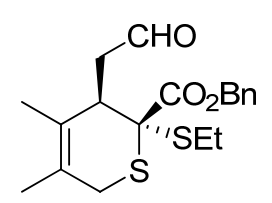

4b

Following the general procedure A ( 5 mol\% $3, r t, 48 \mathrm{~h}$ ), cycloadduct $4 \mathrm{~b}$ was obtained after FC on silica gel (pentane/EtOAc 9:1) in 95\% yield and 91:9 dr as a pale yellow oil. $[\boldsymbol{\alpha}]_{\mathbf{D}}^{20}=-$ $214.9\left(c 0.8, \mathrm{CH}_{2} \mathrm{Cl}_{2}\right) .{ }^{1} \mathrm{H}$ NMR $\left(400 \mathrm{MHz}, \mathrm{CDCl}_{3}\right): \delta \mathrm{ppm} 9.60(\mathrm{t}, J=2.3 \mathrm{~Hz}, 1 \mathrm{H}), 7.41-7.31$ (m, $5 \mathrm{H}), 5.20(\mathrm{~d}, J=12.1 \mathrm{~Hz}, 1 \mathrm{H}), 5.13(\mathrm{~d}, J=12.1 \mathrm{~Hz}, 1 \mathrm{H}), 3.35(\mathrm{~d}, J=17.6 \mathrm{~Hz}, 1 \mathrm{H}), 3.10$ (t, $J=4.2 \mathrm{~Hz}, 1 \mathrm{H}$ ), $2.76(\mathrm{~d}, J=16.6 \mathrm{~Hz}, 1 \mathrm{H}), 2.63(\mathrm{dq}, J=12.5 \mathrm{~Hz}, 7.6,1 \mathrm{H}), 2.56$ (ddd, $J=17.6$, 5.0, $2.4 \mathrm{~Hz}, 1 \mathrm{H}), 2.45-2.37(\mathrm{~m}, 2 \mathrm{H}), 1.70(\mathrm{~s}, 6 \mathrm{H}), 1.12(\mathrm{t}, J=7.6 \mathrm{~Hz}, 3 \mathrm{H}) .{ }^{13} \mathrm{C} \mathrm{NMR}\left(100 \mathrm{MHz}, \mathrm{CDCl}_{3}\right): \delta \mathrm{ppm}$ 200.1, 169.9, 134.9, 128.7 (2C), 128.6, 128.6 (2C), 126.7, 123.1, 67.8, 64.2, 48.2, 42.2, 30.5, 25.2, 19.8, 19.3, 13.6. HRMS (ESI+) $\mathrm{m} / z$ calcd. for $\mathrm{C}_{19} \mathrm{H}_{24} \mathrm{~S}_{2} \mathrm{O}_{3}[\mathrm{M}+\mathrm{Na}]^{+}: 387.1059$; found: 387.1063 . UPC ${ }^{2}$ : IC-3, first $\mathrm{CO}_{2} / \mathrm{iPrOH}$ 
99:1 (0.5 $\mathrm{min})$, then linear gradient $(10 \% / \mathrm{min})$ from $99: 1$ to $60: 40 \mathrm{CO}_{2} / \mathrm{iPrOH}, 3.0 \mathrm{~mL} \cdot \mathrm{min}^{-1}, 40{ }^{\circ} \mathrm{C}, 120 \mathrm{bar}$; $t_{\text {major }}=4.0 \mathrm{~min} ; \mathrm{t}_{\text {minor }}=5.3 \mathrm{~min}(93 \% \mathrm{ee})$.

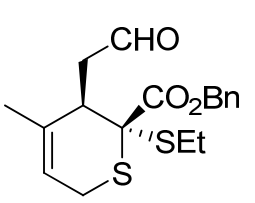

4c

Following the general procedure A (10 mol\% 3, rt, $48 \mathrm{~h})$, cycloadduct $4 \mathrm{c}$ was obtained after FC on silica gel (pentane/EtOAc 9:1) in 82\% yield and 90:10 dr as a pale yellow oil. $[\alpha]_{\mathrm{D}}^{20}=-219.1\left(c 0.4, \mathrm{CH}_{2} \mathrm{Cl}_{2}\right) .{ }^{1} \mathrm{H}$ NMR (400 MHz, CDCl $)$ ): $\delta \mathrm{ppm} 9.60(\mathrm{t}, J=2.1 \mathrm{~Hz}, 1 \mathrm{H})$, 7.41-7.31 (m, 5H), 5.53-5.52 (m, 1H), $5.20(\mathrm{~d}, J=12.1 \mathrm{~Hz}, 1 \mathrm{H}), 5.14(\mathrm{~d}, J=12.1 \mathrm{~Hz}, 1 \mathrm{H})$, 3.40 (ddd, $J=17.4,4.4,2.4 \mathrm{~Hz}, 1 \mathrm{H}), 3.14-3.12(\mathrm{~m}, 1 \mathrm{H}), 2.96-2.90(\mathrm{~m}, 1 \mathrm{H}), 2.68-2.60(\mathrm{~m}$, $1 \mathrm{H}$ ), 2.55 (ddd, $J=17.4,4.4,2.4 \mathrm{~Hz}, 1 \mathrm{H}), 2.50-2.39(\mathrm{~m}, 2 \mathrm{H}), 1.74(\mathrm{~s}, 3 \mathrm{H}), 1.13(\mathrm{t}, J=7.5 \mathrm{~Hz}, 3 \mathrm{H}) .{ }^{13} \mathrm{C}$ NMR (100 MHz, $\mathrm{CDCl}_{3}$ ): $\delta$ ppm 199.7, 169.8, 134.8, 134.7, 128.7 (2C), 128.6, 128.6 (2C), 117.6, 67.8, 64.2, 48.2, 40.3, 25.6, 25.3, 23.6, 13.5. HRMS (ESI+) $\mathrm{m} / \mathrm{z}$ calcd. for $\mathrm{C}_{18} \mathrm{H}_{22} \mathrm{~S}_{2} \mathrm{O}_{3}[\mathrm{M}+\mathrm{Na}]^{+}:$373.0903; found: 373.0907 . UPC $^{2}$ : IC-3, $\mathrm{CO}_{2} / \mathrm{MeCN} 90: 10,3.0 \mathrm{~mL} \cdot \mathrm{min}^{-1}, 40{ }^{\circ} \mathrm{C}, 120 \mathrm{bar}, \mathrm{t}_{\text {major }}=5.4 \mathrm{~min} ; \mathrm{t}_{\text {minor }}=10.1 \mathrm{~min}$ (93\% ee).

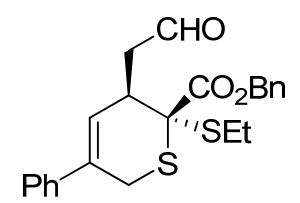

4d

Following the general procedure A (10 mol\% 3, rt, $48 \mathrm{~h}$ ), cycloadduct $4 \mathrm{~d}$ was obtained after $\mathrm{FC}$ on silica gel (pentane/EtOAc 9:1) in 78\% yield and $>95: 5 \mathrm{dr}$ as a pale yellow oil. $[\alpha]_{\mathrm{D}}^{\mathbf{2 0}}=-191.0\left(\mathrm{c} 0.8, \mathrm{CH}_{2} \mathrm{Cl}_{2}\right) .{ }^{1} \mathrm{H}$ NMR $\left(400 \mathrm{MHz}, \mathrm{CDCl}_{3}\right): \delta$ ppm $9.59(\mathrm{~s}, 1 \mathrm{H}), 7.41-7.19$ (m, 10H), $6.09(\mathrm{~d}, J=6.1 \mathrm{~Hz}, 1 \mathrm{H}), 5.24(\mathrm{~d}, J=12.0 \mathrm{~Hz}, 1 \mathrm{H}), 5.17(\mathrm{~d}, J=12.0 \mathrm{~Hz}, 1 \mathrm{H}), 3.70$ (dt, $J=17.1,2.1 \mathrm{~Hz}, 1 \mathrm{H}$ ), 3.38 (ddd, $J=11.2,5.6,3.1 \mathrm{~Hz}, 1 \mathrm{H}), 3.26(\mathrm{~d}, J=17.1 \mathrm{~Hz}, 1 \mathrm{H}$ ), 2.79 (ddd, $J=17.8,8.9,1.5 \mathrm{~Hz}, 1 \mathrm{H}), 2.65(\mathrm{dq}, J=11.9,7.6 \mathrm{~Hz}, 1 \mathrm{H}), 2.52-2.44(\mathrm{~m}, 1 \mathrm{H}), 2.40(\mathrm{dd}, J=17.8,3.3$ $\mathrm{Hz}, 1 \mathrm{H}), 1.11(\mathrm{t}, J=7.5 \mathrm{~Hz}, 3 \mathrm{H}) .{ }^{13} \mathrm{C}$ NMR (100 MHz, CDCl $): \delta \mathrm{ppm} 199.3,169.9,140.7,134.9,133.8,128.7$ (3C), 128.6 (2C), 128.4 (2C), 127.7, 125.7 (2C), 125.6, 67.8, 62.5, 48.9, 37.3, 27.9, 25.2, 13.6. HRMS (ESI+) $\mathrm{m} / \mathrm{z}$ calcd. for $\mathrm{C}_{23} \mathrm{H}_{24} \mathrm{~S}_{2} \mathrm{O}_{3}[\mathrm{M}+\mathrm{Na}]^{+}:$435.1059; found: 435.1061. UPC $\mathbf{C O}^{2}$ IC-3, $\mathrm{CO}_{2} / \mathrm{MeCN} 80: 20,3.0 \mathrm{~mL} \cdot \mathrm{min}^{-1}$, $40^{\circ} \mathrm{C}, 120$ bar, $\mathrm{t}_{\text {major }}=2.6 \mathrm{~min} ; \mathrm{t}_{\text {minor }}=8.5 \mathrm{~min}$ ( $\left.85 \% \mathrm{ee}\right)$.

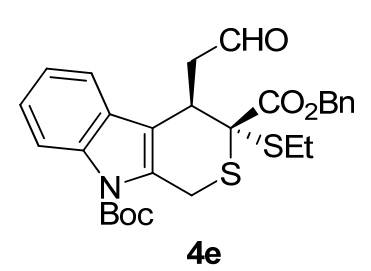

Following the general procedure $\mathrm{A}\left(20 \mathrm{~mol} \% 3,40{ }^{\circ} \mathrm{C}, 48 \mathrm{~h}\right)$, cycloadduct $4 \mathrm{e}$ was obtained after FC on silica gel (pentane/EtOAc 9:1) in 84\% yield and 80:20 dr as a pale yellow oil. $[\boldsymbol{\alpha}]_{\mathrm{D}}^{20}=-187.9\left(\mathrm{c} 1.3, \mathrm{CH}_{2} \mathrm{Cl}_{2}\right.$ ). ${ }^{1} \mathrm{H}$ NMR $\left(400 \mathrm{MHz}, \mathrm{CDCl}_{3}\right): \delta \mathrm{ppm}$ 9.88* (t, $\left.J=1.4 \mathrm{~Hz}, 1 \mathrm{H}^{*}\right), 9.62(\mathrm{t}, J=2.0 \mathrm{~Hz}, 1 \mathrm{H}), 8.08^{+}\left(\mathrm{t}, J=7.5 \mathrm{~Hz}, 1 \mathrm{H}, 1 \mathrm{H}^{*}\right), 7.52-$ $7.00^{+}\left(\mathrm{m}, 8 \mathrm{H}, 8 \mathrm{H}^{*}\right), 5.29(\mathrm{~d}, J=12.0 \mathrm{~Hz}, 1 \mathrm{H}), 5.22(\mathrm{~d}, J=12.0 \mathrm{~Hz}, 1 \mathrm{H}), 5.12^{*}(\mathrm{~d}, J=$ $12.0 \mathrm{~Hz}, 1 \mathrm{H}), 4.95^{*}(\mathrm{~d}, J=12.0 \mathrm{~Hz}, 1 \mathrm{H}), 4.67-4.61 *\left(\mathrm{~m}, 1 \mathrm{H}^{*}\right), 4.27(\mathrm{t}, J=5.0 \mathrm{~Hz}, 1 \mathrm{H})$, $4.18(\mathrm{~s}, 2 \mathrm{H}), 4.14^{*}\left(\mathrm{~d}, J=17.6 \mathrm{~Hz}, 1 \mathrm{H}^{*}\right), 3.95^{*}(\mathrm{~d}, J=17.6 \mathrm{~Hz}, 1 \mathrm{H}), 3.37^{*}$ (ddd, $J=17.9,6.7,1.5 \mathrm{~Hz}, 1 \mathrm{H}^{*}$ ), 2.87-2.66 $\left(\mathrm{m}, 3 \mathrm{H}, 2 \mathrm{H}^{*}\right), 2.63-2.55^{*}\left(\mathrm{~m}, 1 \mathrm{H}^{*}\right), 2.46(\mathrm{dq}, J=11.7,7.4 \mathrm{~Hz}, 1 \mathrm{H}), 1.69(\mathrm{~s}, 9 \mathrm{H}), 1.65^{*}\left(\mathrm{~s}, 9 \mathrm{H}^{*}\right)$, 1.18* (t, $\left.J=7.5 \mathrm{~Hz}, 3 \mathrm{H}^{*}\right), 1.09$ (t, $\left.J=7.5 \mathrm{~Hz}, 3 \mathrm{H}\right) .{ }^{13} \mathrm{C}$ NMR $\left(100 \mathrm{MHz}, \mathrm{CDCl}_{3}\right): \delta \mathrm{ppm} \mathrm{200.0*}, 199.4,169.6$, $168.4^{*}, 150.4,150.0^{*}, 135.4,135.4^{*}, 134.9^{*}, 134.7,129.6^{+}\left(1 \mathrm{C}, 1 \mathrm{C}^{*}\right), 128.8$ (2C), 128.7, 128.6 (2C), 128.1$128.0^{+}\left(\mathrm{m}, 1 \mathrm{C}, 6 \mathrm{C}^{*}\right), 124.9^{*}, 124.6,123.0^{*}, 123.0,118.0^{+}\left(1 \mathrm{C}, 1 \mathrm{C}^{*}\right), 117.8^{*}, 117.7,115.7,115.7^{*}, 84.5,84.4^{*}$, 68.1, 67.9*, 64.0 $0^{+}\left(1 \mathrm{C}, 1 \mathrm{C}^{*}\right), 49.2,47.8^{*}, 35.5,34.7^{*}, 28.3$ (3C), 28.2* (3C*) $25.7^{+}\left(1 \mathrm{C}, 1 \mathrm{C}^{*}\right), 25.5,24.7^{*}, 13.8^{*}$, 13.4. HRMS (ESI+) $\mathrm{m} / z$ calcd. for $\mathrm{C}_{28} \mathrm{H}_{31} \mathrm{~S}_{2} \mathrm{O}_{5}[\mathrm{M}+\mathrm{Na}]^{+}: 548.1536$; found: 548.1543 . UPC ${ }^{2}:$ IC-3, $\mathrm{CO}_{2} / \mathrm{MeCN}$ $90: 10,3.0 \mathrm{~mL} \cdot \mathrm{min}^{-1}, 40^{\circ} \mathrm{C}, 120$ bar; major diastereomer: $\mathrm{t}_{\text {major }}=12.4 \mathrm{~min} ; \mathrm{t}_{\text {minor }}=14.3 \mathrm{~min}(92 \% \mathrm{ee})$. 


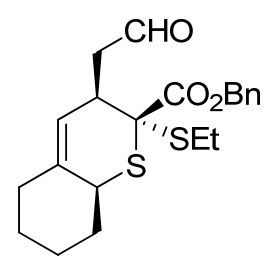

$4 f$

Following the general procedure A ( 5 mol\% 3, rt, 48 h), cycloadduct $4 \mathrm{f}$ was obtained after FC on silica gel (pentane/EtOAc 9:1) in 84\% yield and 86:14 dr as a pale yellow oil. [ $\alpha]_{\mathbf{D}}^{20}=$ -247.2 (c 1.2, $\left.\mathrm{CH}_{2} \mathrm{Cl}_{2}\right) .{ }^{1} \mathrm{H}$ NMR (400 MHz, $\left.\mathrm{CDCl}_{3}\right)$ : $\delta \mathrm{ppm} 9.63(\mathrm{~s}, 1 \mathrm{H}), 7.43-7.28(\mathrm{~m}, 5 \mathrm{H})$, $5.54(\mathrm{~d}, J=6.6 \mathrm{~Hz}, 1 \mathrm{H}), 5.24(\mathrm{~d}, J=12.1 \mathrm{~Hz}, 1 \mathrm{H}), 5.17(\mathrm{~d}, J=12.1 \mathrm{~Hz}, 1 \mathrm{H}), 3.44-3.35(\mathrm{~m}$, $1 \mathrm{H}), 3.26-3.17(\mathrm{~m}, 1 \mathrm{H}), 2.82-2.63(\mathrm{~m}, 2 \mathrm{H}), 2.54-2.41(\mathrm{~m}, 1 \mathrm{H}), 2.35(\mathrm{dd}, J=17.4,3.4 \mathrm{~Hz}$, $1 \mathrm{H}), 2.27-2.18(\mathrm{~m}, 1 \mathrm{H}), 2.10-1.93(\mathrm{~m}, 2 \mathrm{H}), 1.87-1.67(\mathrm{~m}, 2 \mathrm{H}), 1.48-1.20(\mathrm{~m}, 3 \mathrm{H}), 1.14(\mathrm{t}$, $J=7.5 \mathrm{~Hz}, 3 \mathrm{H}) .{ }^{13} \mathrm{C}$ NMR $\left(100 \mathrm{MHz}, \mathrm{CDCl}_{3}\right): \delta \mathrm{ppm} 199.9,169.8,137.8,135.0,128.6(3 \mathrm{C}), 128.6(2 \mathrm{C}), 120.5$, $67.6,63.3,49.4,40.5,37.1,35.5,32.3,27.1,26.0,25.2,13.5$. HRMS (ESI+) $\mathrm{m} / z$ calcd. for $\mathrm{C}_{21} \mathrm{H}_{26} \mathrm{~S}_{2} \mathrm{O}_{3}[\mathrm{M}+\mathrm{Na}]^{+}$: 413.1216; found: 413.1218. UPC ${ }^{2}$ IC-3, first $\mathrm{CO}_{2} / \mathrm{iPrOH} 99: 1$ (0.5 min), then linear gradient $(10 \% / \mathrm{min})$ from 99:1 to $60: 40 \mathrm{CO}_{2} / \mathrm{PrOH}, 3.0 \mathrm{~mL} \cdot \mathrm{min}^{-1}, 40{ }^{\circ} \mathrm{C}, 120$ bar; $\mathrm{t}_{\text {major }}=4.1 \mathrm{~min} ; \mathrm{t}_{\text {minor }}=4.3 \mathrm{~min}$ ( $90 \%$ ee).<smiles>CCOC(=O)[C@]1(SCC)SCC=C[C@@H]1CC=O</smiles>

$4 g$

Following the general procedure A (10 mol\% $\left.3,40{ }^{\circ} \mathrm{C}, 48 \mathrm{~h}\right)$, cycloadduct $4 \mathrm{~g}$ was obtained after FC on silica gel (pentane/EtOAc 9:1) in $82 \%$ yield and 90:10 dr as a pale yellow oil. $[\alpha]_{\mathbf{D}}^{20}=-198.7\left(c 0.4, \mathrm{CH}_{2} \mathrm{Cl}_{2}\right) .{ }^{1} \mathrm{H}$ NMR $\left(400 \mathrm{MHz}, \mathrm{CDCl}_{3}\right): \delta \mathrm{ppm} 9.61(\mathrm{t}, J=1.4 \mathrm{~Hz}, 1 \mathrm{H})$, 7.44-7.30 (m, 5H), 5.90-5.83 (m, 1H), 5.81-5.73 (m, 1H), $5.26(\mathrm{~d}, J=12.1 \mathrm{~Hz}, 1 \mathrm{H}), 5.20(\mathrm{~d}, J$ $=12.1 \mathrm{~Hz}, 1 \mathrm{H}), 3.41(\mathrm{dq}, J=17.6,2.3 \mathrm{~Hz}, 1 \mathrm{H}), 3.26-3.19(\mathrm{~m}, 1 \mathrm{H}), 2.93$ (ddd, $J=17.6,5.1,1.3$ $\mathrm{Hz}, 1 \mathrm{H}), 2.75(\mathrm{ddd}, J=17.6,8.9,1.7 \mathrm{~Hz}, 1 \mathrm{H}), 2.71-2.62(\mathrm{~m}, 1 \mathrm{H}), 2.48(\mathrm{dq}, J=11.8,7.4 \mathrm{~Hz}, 1 \mathrm{H}), 2.38(\mathrm{ddd}, J=$ 17.6, 3.5, $1.1 \mathrm{~Hz}, 1 \mathrm{H}), 1.15(\mathrm{t}, J=7.5 \mathrm{~Hz}, 3 \mathrm{H}) .{ }^{13} \mathrm{C} \mathrm{NMR}\left(100 \mathrm{MHz}, \mathrm{CDCl}_{3}\right): \delta \mathrm{ppm}$ 199.3, 169.9, 134.9, 128.7, 128.6 (2C), 128.6 (2C), 128.2, 123.2, 67.7, 62.6, 48.8, 36.2, 25.8, 25.1, 13.6. HRMS (ESI+) $\mathrm{m} / \mathrm{z}$ calcd. for $\mathrm{C}_{17} \mathrm{H}_{20} \mathrm{~S}_{2} \mathrm{O}_{3}[\mathrm{M}+\mathrm{Na}]^{+}:$359.0746; found: 359.0746. UPC ${ }^{2}$ : IC-3, first $\mathrm{CO}_{2} / \mathrm{PrOH} 99: 1$ (0.5 min), then linear gradient $(10 \% / \mathrm{min})$ from $99: 1$ to $60: 40 \mathrm{CO}_{2} / \mathrm{PrOH}, 3.0 \mathrm{~mL} \cdot \mathrm{min}^{-1}, 40{ }^{\circ} \mathrm{C}, 120$ bar; $\mathrm{t}_{\text {major }}=3.6 \mathrm{~min} ; \mathrm{t}_{\text {minor }}=3.8$ $\min (91 \%$ ee).

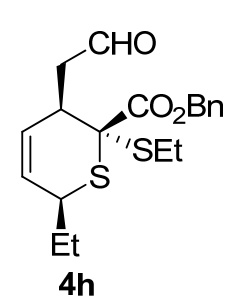

Following the general procedure $\mathrm{A}\left(20 \mathrm{~mol} \% 3,40{ }^{\circ} \mathrm{C}, 48 \mathrm{~h}\right)$, cycloadduct $4 \mathrm{~h}$ was obtained after FC on silica gel (pentane/EtOAc 9:1) in 62\% yield and 85:8:7 dr as a pale yellow oil. $[\alpha]_{\mathrm{D}}^{20}=-127.7\left(\mathrm{c} 0.3, \mathrm{CH}_{2} \mathrm{Cl}_{2}\right) .{ }^{1} \mathrm{H}$ NMR $\left(400 \mathrm{MHz}, \mathrm{CDCl}_{3}\right): \delta \mathrm{ppm} 9.60(\mathrm{~s}, 1 \mathrm{H}), 7.48-7.28(\mathrm{~m}$, $5 \mathrm{H}), 5.85$ (ddd, $J=10.7,6.0,2.3 \mathrm{~Hz}, 1 \mathrm{H}), 5.66(\mathrm{~d}, J=10.7 \mathrm{~Hz}, 1 \mathrm{H}), 5.26(\mathrm{~d}, J=12.1 \mathrm{~Hz}, 1 \mathrm{H}$ ), $5.19(\mathrm{~d}, J=12.1 \mathrm{~Hz}, 1 \mathrm{H}), 3.60-3.47(\mathrm{~m}, 1 \mathrm{H}), 3.26-3.14(\mathrm{~m}, 1 \mathrm{H}), 2.78-2.61(\mathrm{~m}, 2 \mathrm{H}), 2.55-2.42$ $(\mathrm{m}, 1 \mathrm{H}), 2.36(\mathrm{dd}, J=17.6,3.2 \mathrm{~Hz}, 1 \mathrm{H}), 1.75-1.61(\mathrm{~m}, 2 \mathrm{H}), 1.14(\mathrm{t}, J=7.5 \mathrm{~Hz}, 3 \mathrm{H}), 1.02(\mathrm{t}, J=$ $7.4 \mathrm{~Hz}, 3 \mathrm{H}) .{ }^{13} \mathrm{C} \mathrm{NMR}\left(100 \mathrm{MHz}, \mathrm{CDCl}_{3}\right): \delta$ ppm 199.4, 169.9, 134.9, 128.7 (2C), $128.6(2 \mathrm{C}), 128.6(2 \mathrm{C}), 127.6$, $67.7,63.8,49.2,41.2,36.3,26.5,25.3,13.5,11.2$. HRMS (ESI+) $\mathrm{m} / z$ calcd. for $\mathrm{C}_{19} \mathrm{H}_{24} \mathrm{~S}_{2} \mathrm{O}_{3}[\mathrm{M}+\mathrm{Na}]^{+}: 387.1059$; found: $387.1060 . \mathrm{UPC}^{2}$ : IC-3, $\mathrm{CO}_{2} / \mathrm{MeCN} 90: 10,3.0 \mathrm{~mL} \cdot \mathrm{min}^{-1}, 40{ }^{\circ} \mathrm{C}, 120 \mathrm{bar} ; \mathrm{t}_{\text {major }}=2.9 \mathrm{~min} ; \mathrm{t}_{\text {minor }}=4.9 \mathrm{~min}$ (85\% ee).

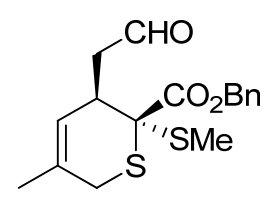

4i

Following the general procedure A (20 mol\% 3, rt, $24 \mathrm{~h}$ ), cycloadduct 4i was obtained after FC on silica gel (pentane/EtOAc 9:1) in 73\% yield and 92:8 dr as a pale yellow oil. $[\boldsymbol{\alpha}]_{\mathbf{D}}^{20}=-163.4\left(c 0.6, \mathrm{CH}_{2} \mathrm{Cl}_{2}\right) .{ }^{1} \mathrm{H}$ NMR $\left(400 \mathrm{MHz}, \mathrm{CDCl}_{3}\right): \delta \mathrm{ppm} 9.62(\mathrm{t}, J=1.4 \mathrm{~Hz}, 1 \mathrm{H})$, 7.45-7.30 (m, 5H), $5.56(\mathrm{~d}, J=4.4 \mathrm{~Hz}, 1 \mathrm{H}), 5.25(\mathrm{~d}, J=12.1 \mathrm{~Hz}, 1 \mathrm{H}), 5.19(\mathrm{~d}, J=12.1 \mathrm{~Hz}$, $1 \mathrm{H}), 3.29(\mathrm{~d}, J=17.1 \mathrm{~Hz}, 1 \mathrm{H}), 3.25-3.18(\mathrm{~m}, 1 \mathrm{H}), 2.79-2.69(\mathrm{~m}, 2 \mathrm{H}), 2.36$ (ddd, $J=17.5$, 3.4, $0.9 \mathrm{~Hz}, 1 \mathrm{H}), 2.04(\mathrm{~s}, 3 \mathrm{H}), 1.72(\mathrm{~s}, 3 \mathrm{H}) .{ }^{13} \mathrm{C}$ NMR (100 MHz, $\left.\mathbf{C D C l}_{3}\right): \delta$ ppm 199.7, 169.5, 135.0, 130.6, 128.6, 128.6 (2C), 128.6 (2C), 122.5, 67.7, 62.7, 49.1, 36.6, 29.2, 23.8, 13.9. HRMS (ESI+) $\mathrm{m} / \mathrm{z}$ calcd. for $\mathrm{C}_{17} \mathrm{H}_{20} \mathrm{~S}_{2} \mathrm{O}_{3}[\mathrm{M}+\mathrm{Na}]^{+}: 359.0746$; found: 359.0752. UPC ${ }^{2}$ : IC-3, $\mathrm{CO}_{2} / \mathrm{MeCN} 90: 10,2.5 \mathrm{~mL} \cdot \mathrm{min}^{-1} ; 55^{\circ} \mathrm{C}, 120 \mathrm{bar}$, $t_{\text {major }}=5.9 \mathrm{~min} ; \mathrm{t}_{\text {minor }}=7.7 \mathrm{~min}(84 \%$ ee). 


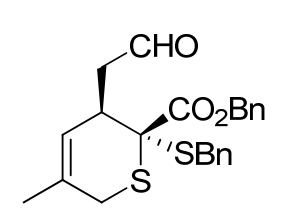

4j

Following the general procedure A (20 mol\% 3, rt, 24 h), cycloadduct $4 \mathbf{j}$ was obtained after FC on silica gel (pentane/EtOAc 9:1) in 73\% yield and 90:10 dr as a pale yellow oil. $[\boldsymbol{\alpha}]_{\mathbf{D}}^{20}=-179.4\left(c \quad 0.6, \mathrm{CH}_{2} \mathrm{Cl}_{2}\right) .{ }^{1} \mathrm{H}$ NMR $\left(400 \mathrm{MHz}^{\mathrm{C}} \mathrm{CDCl}_{3}\right): \delta \mathrm{ppm} 9.59(\mathrm{t}, J=1.4 \mathrm{~Hz}, 1 \mathrm{H})$, 7.40-7.31 (m, 5H), 7.29-7.17 (m, 5H), $5.52(\mathrm{~d}, J=4.3 \mathrm{~Hz}, 1 \mathrm{H}), 5.07(\mathrm{~d}, J=12.0 \mathrm{~Hz}, 1 \mathrm{H})$, $4.98(\mathrm{~d}, J=12.0 \mathrm{~Hz}, 1 \mathrm{H}), 3.86(\mathrm{~d}, J=12.4 \mathrm{~Hz}, 1 \mathrm{H}), 3.65(\mathrm{~d}, J=12.4 \mathrm{~Hz}, 1 \mathrm{H}), 3.34(\mathrm{~d}, J=17.1$ $\mathrm{Hz}, 1 \mathrm{H}), 3.25-3.16(\mathrm{~m}, 1 \mathrm{H}), 2.82-2.67(\mathrm{~m}, 2 \mathrm{H}), 2.33$ (ddd, $J=17.4,3.5,1.0 \mathrm{~Hz}, 1 \mathrm{H}), 1.72(\mathrm{~s}, 3 \mathrm{H}) .{ }^{13} \mathrm{C}$ NMR

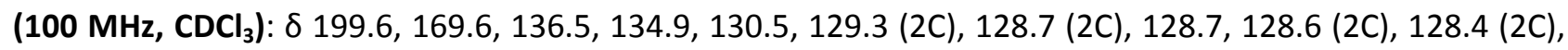
127.1, 122.5, 67.7, 63.2, 48.9, 36.8, 35.7, 29.4, 23.8. HRMS (ESI+) $\mathrm{m} / \mathrm{z}$ calcd. for $\mathrm{C}_{23} \mathrm{H}_{24} \mathrm{~S}_{2} \mathrm{O}_{3}[\mathrm{M}+\mathrm{Na}]^{+}$: 435.1059; found: 435.1065. UPC ${ }^{2}: \mathrm{IC}-3, \mathrm{CO}_{2} / \mathrm{MeCN} 90: 10,3.0 \mathrm{~mL} \cdot \mathrm{min}^{-1}, 40^{\circ} \mathrm{C}, 120$ bar; $\mathrm{t}_{\text {major }}=9.4 \mathrm{~min} ; \mathrm{t}_{\text {minor }}$ $=12.8 \mathrm{~min}(87 \% \mathrm{ee})$.

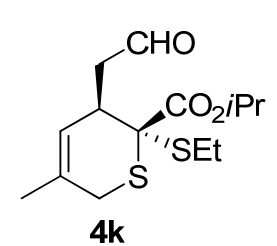

$(\mathrm{t}, J=7.5 \mathrm{~Hz}, 3 \mathrm{H}) .{ }^{13} \mathrm{C}$ NMR $\left(100 \mathrm{MHz}, \mathrm{CDCl}_{3}\right): \delta \mathrm{ppm} 200.0,169.6,130.6,122.7,70.3,62.7,48.9,36.9,29.5$, 25.1, 23.8, 21.6, 21.5, 13.8. HRMS (ESI+) $\mathrm{m} / \mathrm{z}$ calcd. for $\mathrm{C}_{14} \mathrm{H}_{22} \mathrm{~S}_{2} \mathrm{O}_{3}[\mathrm{M}+\mathrm{Na}]^{+}:$325.0903; found: 325.0905 . UPC $^{2}$ : IC-3, $\mathrm{CO}_{2} / \mathrm{MeCN} 90: 10,3.0 \mathrm{~mL} \cdot \mathrm{min}^{-1}, 40^{\circ} \mathrm{C}, 120$ bar; $\mathrm{t}_{\text {major }}=2.4 \mathrm{~min} ; \mathrm{t}_{\text {minor }}=3.7 \mathrm{~min}$ (94\% ee).

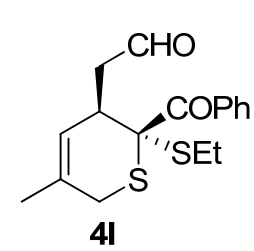

Following the general procedure A ( $20 \mathrm{~mol} \% 3, \mathrm{rt}, 48 \mathrm{~h}$ ), cycloadduct $4 \mathrm{l}$ was obtained after FC on silica gel (pentane/EtOAc 9:1 to 4:1) in 62\% yield and 65:35 dr as a pale yellow oil. $[\boldsymbol{\alpha}]_{\mathrm{D}}^{\mathbf{2 0}}=-8.0\left(\mathrm{c} 1.5, \mathrm{CH}_{2} \mathrm{Cl}_{2}\right) .{ }^{1} \mathrm{H}$ NMR $\left(400 \mathrm{MHz}, \mathrm{CDCl}_{3}\right): \delta \mathrm{ppm} 9.86^{*}\left(\mathrm{~s}, 1 \mathrm{H}^{*}\right), 9.50(\mathrm{~s}, 1 \mathrm{H})$, $8.31(\mathrm{~d}, J=7.4 \mathrm{~Hz}, 2 \mathrm{H}), 8.14^{*}\left(\mathrm{~d}, J=8.0 \mathrm{~Hz}, 2 \mathrm{H}^{*}\right), 7.56-7.33^{+}\left(\mathrm{m}, 3 \mathrm{H}, 3 \mathrm{H}^{*}\right), 5.61(\mathrm{~d}, J=6.2$ $\mathrm{Hz}, 1 \mathrm{H}), 5.50^{*}\left(\mathrm{~d}, J=6.5 \mathrm{~Hz}, 1 \mathrm{H}^{*}\right), 3.67-3.59^{*}\left(\mathrm{~m}, 1 \mathrm{H}^{*}\right), 3.55-3.45(\mathrm{~m}, 1 \mathrm{H}), 3.38(\mathrm{~d}, J=$ $17.6 \mathrm{~Hz}, 1 \mathrm{H}), 3.24^{*}\left(\mathrm{dd}, J=17.5,5.1 \mathrm{~Hz}, 1 \mathrm{H}^{*}\right), 3.08^{*}\left(\mathrm{~d}, J=17.5 \mathrm{~Hz}, 1 \mathrm{H}^{*}\right), 2.86-2.41^{+}(\mathrm{m}, 4 \mathrm{H}, 4 \mathrm{H}), 2.25(\mathrm{dd}, J$ $=17.6,3.0 \mathrm{~Hz}, 1 \mathrm{H}), 1.77(\mathrm{~s}, 3 \mathrm{H}), 1.62^{*}\left(\mathrm{~s}, 3 \mathrm{H}^{*}\right), 1.16(\mathrm{t}, J=7.4 \mathrm{~Hz}, 3 \mathrm{H}), 1.14^{*}\left(\mathrm{t}, J=7.2 \mathrm{~Hz}, 3 \mathrm{H}^{*}\right) .{ }^{13} \mathrm{C}$ NMR

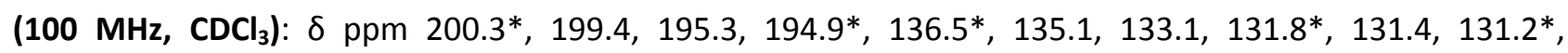
129.7(2C), 129.4*(2C*), 128.4(2C), 127.7*(2C*), 125.4*, 122.6, 69.1, 63.4*, 48.6, 47.7*, 37.5, 35.4*, 31.4*, 29.5, 25.1, 24.2*, 23.9*, 23.8, 13.7, 13.6*. HRMS (ESI+) $\mathrm{m} / \mathrm{z}$ calcd. for $\mathrm{C}_{17} \mathrm{H}_{20} \mathrm{~S}_{2} \mathrm{O}_{2}[\mathrm{M}+\mathrm{Na}]^{+}:$343.0797; found: 343.0800. UPC ${ }^{2}$ : IC-3, $\mathrm{CO}_{2} / \mathrm{MeCN} 70: 30,3.0 \mathrm{~mL} \cdot \mathrm{min}^{-1}$; major diastereomer: $\mathrm{t}_{\text {major }}=1.3 \mathrm{~min} ; \mathrm{t}_{\text {minor }}=1.5 \mathrm{~min}(6 \%$ ee); minor diastereomer: $\mathrm{t}_{\text {major }}=1.0 \mathrm{~min} ; \mathrm{t}_{\text {minor }}=1.6 \mathrm{~min}(53 \% \mathrm{ee})$.

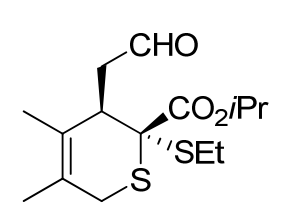

$4 \mathrm{~m}$

Following the general procedure A (20 mol\% 3, rt, $24 \mathrm{~h}$ ), cycloadduct $4 \mathrm{~m}$ was obtained after FC on silica gel (pentane/EtOAc 9:1) in 78\% yield and 92:8 dr as a pale yellow oil. $[\boldsymbol{\alpha}]_{\mathbf{D}}^{20}=-240.5\left(c 0.5, \mathrm{CH}_{2} \mathrm{Cl}_{2}\right) .{ }^{1} \mathrm{H}$ NMR $\left(400 \mathrm{MHz}, \mathrm{CDCl}_{3}\right): \delta \mathrm{ppm} 9.77(\mathrm{t}, J=2.2 \mathrm{~Hz}, 1 \mathrm{H})$, 5.05 (hept, $J=6.3 \mathrm{~Hz}, 1 \mathrm{H}), 3.36(\mathrm{~d}, J=17.0 \mathrm{~Hz}, 1 \mathrm{H}), 3.10(\mathrm{t}, J=4.5 \mathrm{~Hz}, 1 \mathrm{H}), 2.81-2.42(\mathrm{~m}$, $5 \mathrm{H}), 1.71(\mathrm{~s}, 6 \mathrm{H}), 1.29(\mathrm{~d}, J=6.3 \mathrm{~Hz}, 3 \mathrm{H}), 1.27(\mathrm{~d}, J=6.4 \mathrm{~Hz}, 3 \mathrm{H}), 1.22(\mathrm{t}, J=7.5 \mathrm{~Hz}, 3 \mathrm{H})$. ${ }^{13} \mathrm{C}$ NMR (100 MHz, $\mathrm{CDCl}_{3}$ ): $\delta$ ppm 200.4, 169.6, 126.8, 123.0, 70.5, 64.2, 48.3, 42.1, 30.6, 25.2, 21.5 (2C), 19.8, 19.3, 13.8. HRMS (ESI+) $\mathrm{m} / z$ calcd. for $\mathrm{C}_{15} \mathrm{H}_{24} \mathrm{~S}_{2} \mathrm{O}_{3}[\mathrm{M}+\mathrm{Na}]^{+}:$339.1059; found: 339.1065. UPC ${ }^{2}$ : IC-3, $\mathrm{CO}_{2} / \mathrm{MeCN} 90: 10,3.0 \mathrm{~mL} \cdot \mathrm{min}^{-1}, 40{ }^{\circ} \mathrm{C}, 120 \mathrm{bar}, \mathrm{t}_{\text {major }}=2.4 \mathrm{~min} ; \mathrm{t}_{\text {minor }}=7.4 \mathrm{~min}$ (97\% ee). 


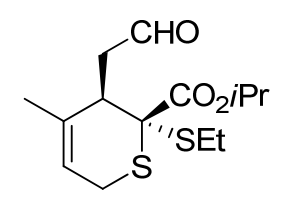

$4 n$

Following the general procedure A (20 mol\% 3, rt, $24 \mathrm{~h}$ ), cycloadduct 4n was obtained after FC on silica gel (pentane/EtOAc 9:1 to 4:1) in 76\% yield and 90:10 dr as a pale yellow oil. $[\alpha]_{\mathbf{D}}^{20}=-252.9\left(c 0.7, \mathrm{CH}_{2} \mathrm{Cl}_{2}\right) .{ }^{1} \mathbf{H} \mathbf{N M R}\left(400 \mathrm{MHz}, \mathrm{CDCl}_{3}\right): \delta \mathrm{ppm} 9.79(\mathrm{t}, J=2.0$ $\mathrm{Hz}, 1 \mathrm{H}$ ), 5.56-5.50 (m, 1H), 5.06 (hept, $J=6.3 \mathrm{~Hz}, 1 \mathrm{H}), 3.45-3.36(\mathrm{~m}, 1 \mathrm{H}), 3.15-3.10(\mathrm{~m}$, $1 \mathrm{H}$ ), 2.93 (ddd, $J=17.4,5.2,1.5 \mathrm{~Hz}, 1 \mathrm{H}$ ), 2.80-2.46 (m, 4H), $1.76(\mathrm{~s}, 3 \mathrm{H}), 1.29$ (d, $J=6.2$

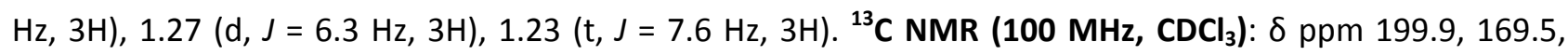
$134.9,117.6,70.5,64.2,48.3,40.2,25.6,25.3,23.6,21.5$ (2C), 13.8. HRMS (ESI+) m/z calcd. for $\mathrm{C}_{14} \mathrm{H}_{22} \mathrm{~S}_{2} \mathrm{O}_{3}$ $[\mathrm{M}+\mathrm{Na}]^{+}:$325.0903; found: $325.0905 . \mathrm{UPC}^{2}: \mathrm{IC}-3, \mathrm{CO}_{2} / \mathrm{MeCN} 90: 10,3.0 \mathrm{~mL} \cdot \mathrm{min}^{-1}, 40^{\circ} \mathrm{C}, 120$ bar; $\mathrm{t}_{\text {major }}=2.9$ $\min ; t_{\text {minor }}=4.7 \mathrm{~min}$ (94\% ee).

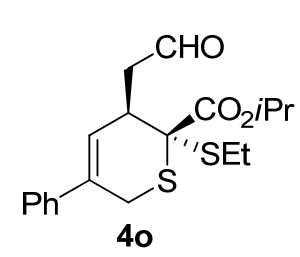

Following the general procedure A (10 mol\% 3, rt, $48 \mathrm{~h}$ ), cycloadduct 40 was obtained after FC on silica gel (pentane/EtOAc 9:1) in 70\% yield and $>98: 2 \mathrm{dr}$ as a pale yellow oil. $[\boldsymbol{\alpha}]_{\mathbf{D}}^{\mathbf{2 0}}=-230.7\left(\mathrm{c} 0.5, \mathrm{CH}_{2} \mathrm{Cl}_{2}\right) .{ }^{1} \mathrm{H}$ NMR $\left(400 \mathrm{MHz}, \mathrm{CDCl}_{3}\right): \delta \mathrm{ppm} 9.83(\mathrm{t}, J=1.4 \mathrm{~Hz}, 1 \mathrm{H})$, 7.36-7.26 (m, 5H), 6.18-6.14 (m, 1H), 5.11 (hept, $J=6.3 \mathrm{~Hz}, 1 \mathrm{H}), 3.77$ (dt, $J=17.1,2.1$ $\mathrm{Hz}, 1 \mathrm{H}), 3.46-3.39(\mathrm{~m}, 1 \mathrm{H}), 3.33(\mathrm{~d}, J=17.0 \mathrm{~Hz}, 1 \mathrm{H}), 2.93(\mathrm{ddd}, J=17.6,8.8,1.7 \mathrm{~Hz}, 1 \mathrm{H}$ ), 2.82-2.61 (m, 3H), $1.32(\mathrm{~d}, J=6.3 \mathrm{~Hz}, 3 \mathrm{H}), 1.31(\mathrm{~d}, J=6.3 \mathrm{~Hz}, 3 \mathrm{H}), 1.25(\mathrm{t}, J=7.5 \mathrm{~Hz}, 3 \mathrm{H}) .{ }^{13} \mathrm{C} \mathrm{NMR}(100 \mathrm{MHz}$, $\mathrm{CDCl}_{3}$ ): $\delta$ ppm 199. 6, 169.5, 140.8, 133.9, 128.5 (2C), 127.7, 125.7 (3C), 70.4, 62.5, 49.0, 37.3, 28.0, 25.2, 21.6, 21.6, 13.8. HRMS (ESI+) $\mathrm{m} / \mathrm{z}$ calcd. for $\mathrm{C}_{19} \mathrm{H}_{24} \mathrm{~S}_{2} \mathrm{O}_{3}[\mathrm{M}+\mathrm{Na}]^{+}$: 387.1059; found: 387.1061. UPC ${ }^{2}$ : IC-3, $\mathrm{CO}_{2} / \mathrm{MeCN} 90: 10,3.0 \mathrm{~mL} \cdot \mathrm{min}^{-1}, 40^{\circ} \mathrm{C}, 120 \mathrm{bar}, \mathrm{t}_{\text {major }}=3.7 \mathrm{~min} ; \mathrm{t}_{\text {minor }}=17.3 \mathrm{~min}$ (95\% ee).

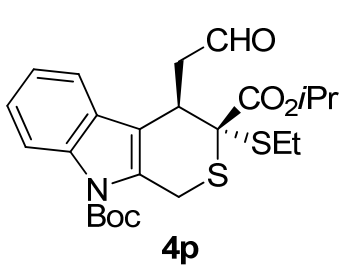

Following the general procedure $A\left(20 \mathrm{~mol} \% 3,40{ }^{\circ} \mathrm{C}, 48 \mathrm{~h}\right)$, cycloadduct $4 \mathrm{p}$ was obtained after FC on silica gel (pentane/EtOAc 9:1 to 4:1) in 84\% yield and 93:7 dr as a pale yellow oil. $[\boldsymbol{\alpha}]_{\mathbf{D}}^{20}=-212.9\left(c 0.8, \mathrm{CH}_{2} \mathrm{Cl}_{2}\right) .{ }^{1} \mathrm{H} \mathbf{N M R}\left(400 \mathrm{MHz}, \mathrm{CDCl}_{3}\right): \delta \mathrm{ppm}$ $9.78(\mathrm{t}, J=1.9 \mathrm{~Hz}, 1 \mathrm{H}), 8.08(\mathrm{~d}, J=7.9 \mathrm{~Hz}, 1 \mathrm{H}), 7.47(\mathrm{~d}, J=7.8 \mathrm{~Hz}, 1 \mathrm{H}), 7.32-7.20(\mathrm{~m}$, $2 \mathrm{H}$ ), 5.12 (hept, $J=6.3 \mathrm{~Hz}, 1 \mathrm{H}$ ), $4.25(\mathrm{t}, J=4.9 \mathrm{~Hz}, 1 \mathrm{H}$ ), $4.18(\mathrm{~s}, 2 \mathrm{H}), 2.90$ (ddd, $J=$ 17.6, 5.0, 2.2 Hz, 1H), 2.85-2.74 (m, 2H), $2.63(\mathrm{dq}, J=11.7,7.4 \mathrm{~Hz}, 1 \mathrm{H}), 1.69(\mathrm{~s}, 9 \mathrm{H})$, $1.34(\mathrm{~d}, J=6.3 \mathrm{~Hz}, 3 \mathrm{H}), 1.34(\mathrm{~d}, J=6.3 \mathrm{~Hz}, 3 \mathrm{H}), 1.19(\mathrm{t}, J=7.5 \mathrm{~Hz}, 3 \mathrm{H}) .{ }^{13} \mathrm{C}$ NMR (100 MHz, CDCl $): \delta 199.6$, 169.2, 150.4, 135.5, 129.6, 128.1, 124.6, 123.0, 118.2, 117.8, 115.7, 84.5, 70.9, 64.0, 49.3, 35.4, 28.3 (3C), 25.7, 25.5, 21.6 (2C), 13.6. HRMS (ESI+) $\mathrm{m} / \mathrm{z}$ calcd. for $\mathrm{C}_{24} \mathrm{H}_{31} \mathrm{NS}_{2} \mathrm{O}_{5}[\mathrm{M}+\mathrm{Na}]^{+}:$500.1536; found: 500.1540 . The ee was determined after transformation into compound $\mathbf{5 a}$.<smiles>CCO[C@]1(SCC)SC(C(Cl)Cl)C=C[C@@H]1CC=O</smiles>

$4 q$

Following the general procedure $A\left(20\right.$ mol\% $\left.3,40{ }^{\circ} \mathrm{C}, 48 \mathrm{~h}\right)$, cycloadduct $4 \mathrm{q}$ was obtained after FC on silica gel (pentane/EtOAc 9:1) in 92\% yield and 86:9:5 dr as a pale yellow oil. $[\alpha]_{\mathbf{D}}^{\mathbf{2 0}}=-130.2\left(c 0.3, \mathrm{CH}_{2} \mathrm{Cl}_{2}\right) .{ }^{1} \mathbf{H}$ NMR (400 MHz, $\left.\mathrm{CDCl}_{3}\right): \delta \mathrm{ppm} 9.78(\mathrm{t}, J=1.5 \mathrm{~Hz}, 1 \mathrm{H}), 5.87$ (ddd, $J=10.7,6.0,2.4 \mathrm{~Hz}, 1 \mathrm{H}$ ), 5.68 (ddd, $J=10.7,2.4,1.3 \mathrm{~Hz}, 1 \mathrm{H}$ ), 5.09 (hept, $J=6.2 \mathrm{~Hz}$, $1 \mathrm{H}), 3.57-3.53(\mathrm{~m}, 1 \mathrm{H}), 3.23-3.18(\mathrm{~m}, 1 \mathrm{H}), 2.81(\mathrm{ddd}, J=17.5,8.8,1.8 \mathrm{~Hz}, 1 \mathrm{H}), 2.77(\mathrm{dq}, J=$ $11.8,7.6 \mathrm{~Hz}, 1 \mathrm{H}), 2.63(\mathrm{dq}, J=11.8,7.6 \mathrm{~Hz}, 1 \mathrm{H}), 2.56(\mathrm{ddd}, J=17.4,3.4,1.0 \mathrm{~Hz}, 1 \mathrm{H}), 1.74-$ $1.64(\mathrm{~m}, 2 \mathrm{H}), 1.30(\mathrm{~d}, J=6.2 \mathrm{~Hz}, 3 \mathrm{H}), 1.28(\mathrm{~d}, J=6.2 \mathrm{~Hz}, 3 \mathrm{H}), 1.23(\mathrm{t}, J=7.6,3 \mathrm{H}), 1.04(\mathrm{t}, J=7.4,3 \mathrm{H}) .{ }^{13} \mathrm{C}$ NMR (100 MHz, $\left.\mathrm{CDCl}_{3}\right): \delta$ 199.6, 169.5, 128.7, 127.6, 70.3, 63.8, 49.2, 41.3, 36.3, 26.6, 25.3, 21.6, 21.5, 13.7, 11.2. HRMS (ESI+) $\mathrm{m} / \mathrm{z}$ calcd. for $\mathrm{C}_{15} \mathrm{H}_{24} \mathrm{~S}_{2} \mathrm{O}_{3}[\mathrm{M}+\mathrm{Na}]^{+}:$339.1059; found: 339.1063. UPC : IC-3, $\mathrm{CO}_{2} / \mathrm{MeCN}$ $90: 10,3.0 \mathrm{~mL} \cdot \mathrm{min}^{-1}, 40^{\circ} \mathrm{C}, 120 \mathrm{bar} ; \mathrm{t}_{\text {major }}=1.4 \mathrm{~min} ; \mathrm{t}_{\text {minor }}=3.1 \mathrm{~min}$ ( $83 \%$ ee) . 


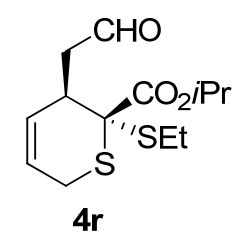

Following the general procedure $A\left(20 \mathrm{~mol} \% 3,40{ }^{\circ} \mathrm{C}, 48 \mathrm{~h}\right.$ ), cycloadduct $4 \mathrm{r}$ was obtained after FC on silica gel (pentane/EtOAc 9:1) in $89 \%$ yield and $92: 8 \mathrm{dr}$ as a pale yellow oil. $[\boldsymbol{\alpha}]_{\mathbf{D}}^{\mathbf{2 0}}$ $=-253.7\left(c 0.3, \mathrm{CH}_{2} \mathrm{Cl}_{2}\right) .{ }^{1} \mathrm{H}$ NMR $\left(400 \mathrm{MHz}, \mathrm{CDCl}_{3}\right): \delta \mathrm{ppm} 9.78(\mathrm{t}, J=1.4 \mathrm{~Hz}, 1 \mathrm{H}), 5.91-5.84$ $(\mathrm{m}, 1 \mathrm{H}), 5.83-5.76(\mathrm{~m}, 1 \mathrm{H}), 5.08$ (hept, $J=6.3 \mathrm{~Hz}, 1 \mathrm{H}), 3.46-3.36(\mathrm{~m}, 1 \mathrm{H}), 3.25-3.17(\mathrm{~m}, 1 \mathrm{H})$, $2.94(\mathrm{dd}, J=17.6,5.1 \mathrm{~Hz}, 1 \mathrm{H}), 2.84$ (ddd, $J=17.5,8.8,1.7 \mathrm{~Hz}, 1 \mathrm{H}), 2.78-2.69(\mathrm{~m}, 1 \mathrm{H}), 2.67-$ $2.53(\mathrm{~m}, 2 \mathrm{H}), 1.30(\mathrm{~d}, J=6.7 \mathrm{~Hz}, 3 \mathrm{H}), 1.27(\mathrm{~d}, J=6.7 \mathrm{~Hz}, 3 \mathrm{H}), 1.23(\mathrm{t}, J=7.5 \mathrm{~Hz}, 3 \mathrm{H}) .{ }^{13} \mathrm{C} \mathbf{~ N M R}(100 \mathrm{MHz}$, $\mathrm{CDCl}_{3}$ ): $\delta$ ppm 199.6, 169.5, 128.2, 123.3, 70.3, 62.5, 48.8, 36.1, 25.8, 25.1, 21.6, 21.5, 13.8. HRMS (ESI+) $\mathrm{m} / \mathrm{z}$ calcd. for $\mathrm{C}_{13} \mathrm{H}_{20} \mathrm{~S}_{2} \mathrm{O}_{3}[\mathrm{M}+\mathrm{Na}]^{+}: 311.0746$; found: 311.0750 . The ee was determined after transformation to compound $\mathbf{5 b}$.

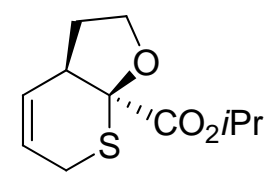

$5 a$

Following the general procedure $\mathrm{B}$, compound 5a was obtained after $\mathrm{FC}$ on silica gel (pentane/EtOAc 5:1) in $72 \%$ yield over two steps. $[\boldsymbol{\alpha}]_{\mathbf{D}}^{\mathbf{2 0}}=-8.0$ (c 1.1, $\mathrm{CHCl}_{3}$ ). ${ }^{1} \mathbf{H}$ NMR (400 MHz, $\mathrm{CDCl}_{3}$ ): $\delta$ ppm 5.99-5.94 (m, 1H), 5.84-5.81 (m, 1H), 5.06 (hept, $J=6.2 \mathrm{~Hz}, 1 \mathrm{H}$ ), $4.07(\mathrm{dd}, J=6.6,2.6 \mathrm{~Hz}, 1 \mathrm{H}), 4.05(\mathrm{dd}, J=6.6,2.6 \mathrm{~Hz}, 1 \mathrm{H}), 3.28-3.22(\mathrm{~m}, 1 \mathrm{H}), 3.20-3.14$ $(\mathrm{m}, 1 \mathrm{H}), 3.10-3.04(\mathrm{~m}, 1 \mathrm{H}), 2.36-2.27(\mathrm{~m}, 1 \mathrm{H}), 2.00-1.91(\mathrm{~m}, 1 \mathrm{H}), 1.29(\mathrm{~d}, J=6.2 \mathrm{~Hz}, 3 \mathrm{H})$, $1.27(\mathrm{~d}, J=6.2 \mathrm{~Hz}, 3 \mathrm{H}) .{ }^{13} \mathrm{C} \mathrm{NMR}\left(100 \mathrm{MHz}, \mathrm{CDCl}_{3}\right): \delta \mathrm{ppm}{ }^{13} \mathrm{C} \mathrm{NMR}\left(101 \mathrm{MHz}, \mathrm{CDCl}_{3}\right) \delta 169.7,129.1,123.6$, 86.7, 69.4, 68.3, 41.8, 32.0, 26.0, 21.6, 21.4. HRMS (ESI+) $\mathrm{m} / z$ calcd. for $\mathrm{C}_{11} \mathrm{H}_{16} \mathrm{SO}_{3}[\mathrm{M}+\mathrm{Na}]^{+}:$251.0712; found: 251.0710. UPC ${ }^{2}$ : IC-3, $\mathrm{CO}_{2} / \mathrm{MeCN} 90: 10,3.0 \mathrm{~mL} \cdot \mathrm{min}^{-1}, 40{ }^{\circ} \mathrm{C}, 120$ bar; $\mathrm{t}_{\text {major }}=3.4 \mathrm{~min} ; \mathrm{t}_{\text {minor }}=4.1 \mathrm{~min}(92 \%$ ee).

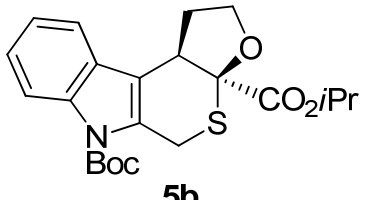

$5 b$

Following the general procedure $\mathrm{B}$, compound $\mathbf{5 b}$ was obtained after FC on silica gel (pentane/EtOAc 9:1 to $4: 1$ ) in $51 \%$ yield over two steps. $[\alpha]_{\mathbf{D}}^{20}=-42.4$ (c 0.9, $\left.\mathrm{CH}_{2} \mathrm{Cl}_{2}\right) .{ }^{1} \mathrm{H}$ NMR $\left(400 \mathrm{MHz}, \mathrm{CDCl}_{3}\right): 8.12(\mathrm{~d}, J=8.1 \mathrm{~Hz}, 1 \mathrm{H}), 7.46(\mathrm{~d}, J=7.4 \mathrm{~Hz}, 1 \mathrm{H})$, 7.38-7.21 (m, 2H), 5.10 (hept, $J=6.2 \mathrm{~Hz}, 1 \mathrm{H}), 4.32-4.08(\mathrm{~m}, 4 \mathrm{H}), 3.93(\mathrm{t}, J=7.3 \mathrm{~Hz}$, $1 \mathrm{H}), 2.78-2.63(\mathrm{~m}, 1 \mathrm{H}), 2.32-2.18(\mathrm{~m}, 1 \mathrm{H}), 1.68(\mathrm{~s}, 9 \mathrm{H}), 1.31(\mathrm{~d}, J=6.2 \mathrm{~Hz}, 3 \mathrm{H})$, $1.28(\mathrm{~d}, J=6.2 \mathrm{~Hz}, 3 \mathrm{H}) .{ }^{13} \mathrm{C} \mathrm{NMR}\left(100 \mathrm{MHz}, \mathrm{CDCl}_{3}\right): \delta 169.5,150.3,135.6,130.8,128.7,124.4,122.7,118.4$, 117.8, 115.7, 88.1, 84.3, 69.8, 69.2, 41.8, 32.0, 28.3 (3C), 26.1, 21.6, 21.5. HRMS (ESI+) $\mathrm{m} / \mathrm{z}$ calcd. for $\mathrm{C}_{22} \mathrm{H}_{27} \mathrm{NSO}_{5}[\mathrm{M}+\mathrm{Na}]^{+}:$440.1502; found: 440.1506. UPC ${ }^{2}$ : IC-3, first $\mathrm{CO}_{2} / \mathrm{MeCN}$ 99:1 (0.5 min), then linear gradient $(10 \% / \mathrm{min})$ from $99: 1$ to $60: 40 \mathrm{CO}_{2} / \mathrm{MeCN}, 3.0 \mathrm{~mL} \cdot \mathrm{min}^{-1}, 40{ }^{\circ} \mathrm{C}, 120$ bar; $\mathrm{t}_{\text {major }}=4.1 \mathrm{~min} ; \mathrm{t}_{\text {minor }}=3.7$ $\min (90 \%$ ee).

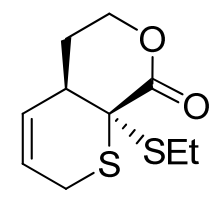

6

To a solution of the aldehyde $4 \mathbf{r}\left(0.35 \mathrm{mmol}, 1\right.$ equiv) in $\mathrm{MeOH}(3 \mathrm{~mL})$ was added $\mathrm{NaBH}_{4}$ ( $0.52 \mathrm{mmol}, 1.5$ equiv) at $0{ }^{\circ} \mathrm{C}$. After stirring for $5 \mathrm{~min}$ the reaction was allowed to reach $\mathrm{rt}$ and stirred for further $10 \mathrm{~min}$. A saturated solution of aq. $\mathrm{NH}_{4} \mathrm{Cl}$ was then added and the resulting solution was extracted three times with $\mathrm{CH}_{2} \mathrm{Cl}_{2}$. The combined organic layers were dried over $\mathrm{Na}_{2} \mathrm{SO}_{4}$ and concentrated in vacuo. The crude alcohol intermediate was redissolved in $\mathrm{CHCl}_{3}(1.5 \mathrm{~mL})$ and $p-\mathrm{TsOH} \cdot \mathrm{H}_{2} \mathrm{O}(0.175 \mathrm{mmol}, 0.5$ equiv) was added. This mixture was heated at $50{ }^{\circ} \mathrm{C}$ for $36 \mathrm{~h}$ in order to reach complete conversion of $4 \mathrm{r}$. The crude reaction mixture was directly purified by FC (85:15 pentane/EtOAc) on silica gel to afford lactone 6 in $59 \%$ yield over two steps as a pale yellow oil. $[\alpha]_{\mathrm{D}}^{20}=-65.3\left(\mathrm{c} 0.9, \mathrm{CHCl}_{3}\right) .{ }^{1} \mathbf{H}$ NMR $\left(400 \mathrm{MHz}, \mathrm{CDCl}_{3}\right): \delta \mathrm{ppm} 6.08-6.03(\mathrm{~m}, 1 \mathrm{H}), 5.67-$ $5.64(\mathrm{~m}, 1 \mathrm{H}), 4.46-4.41(\mathrm{~m}, 1 \mathrm{H}), 4.34$ (ddd, $J=12.3,8.3,4.2 \mathrm{~Hz}, 1 \mathrm{H}), 3.14-3.13(\mathrm{~m}, 2 \mathrm{H}), 3.00(\mathrm{dq}, J=11.6$, $7.1 \mathrm{~Hz}, 1 \mathrm{H}), 2.88-2.80(\mathrm{~m}, 2 \mathrm{H}), 2.53-2.46(\mathrm{~m}, 1 \mathrm{H}), 1.88-1.81(\mathrm{~m}, 1 \mathrm{H}), 1.26(\mathrm{t}, J=7.1 \mathrm{~Hz}, 3 \mathrm{H}) .{ }^{13} \mathrm{C}$ NMR $(100$ 
MHz, $\mathrm{CDCl}_{3}$ ): $\delta$ ppm 167.8, 129.0, 126.2, 68.5, 56.6, 40.8, 27.2, 25.9, 24.8, 13.7. HRMS (ESI+) $\mathrm{m} / \mathrm{z}$ calcd. for $\mathrm{C}_{10} \mathrm{H}_{14} \mathrm{~S}_{2} \mathrm{O}_{2}[\mathrm{M}+\mathrm{Na}]^{+}:$253.0327; found: 253.0327.

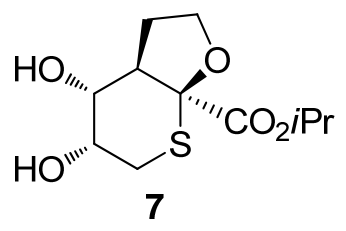

Potassium ferricyanide $(0.79 \mathrm{mmol}$, 3equiv), potassium carbonate $(0.79 \mathrm{mmol}, 3$ equiv), osmium (III) chloride $(1.8 \mu \mathrm{mol})$, quinuclidine $(35 \mu \mathrm{mol})$ and methanesulfonamide $\left(0.26 \mathrm{mmol}, 1\right.$ equiv) were dissolved in a mixture of $\mathrm{H}_{2} \mathrm{O} / \mathrm{t}$ $\mathrm{BuOH}$ (1:1) $2 \mathrm{~mL}$. Then, a solution of $\mathbf{5 a}$ in $2 \mathrm{~mL}$ of the same mixture of solvents was added. The reaction was vigorously stirred for $24 \mathrm{~h}$ at $\mathrm{rt}$. Anhydrous sodium sulfite ( $3.28 \mathrm{mmol}, 12.5$ equiv) was added and the stirring was continued for $30 \mathrm{~min}$. The product 7 was extracted with $\mathrm{CH}_{2} \mathrm{Cl}_{2}$, dried over $\mathrm{Na}_{2} \mathrm{SO}_{4}$ and concentrated in vacuo. The product was purified by FC (3:1 pentane/EtOAC) in $71 \%$ of yield as a colorless oil. $[\alpha]_{\mathrm{D}}^{20}=-55.4$ (c 2, $\mathrm{CHCl}_{3}$ ). ${ }^{1} \mathbf{H}$ NMR $\left(400 \mathbf{~ M H z}, \mathrm{CDCl}_{3}\right.$ ) $\delta$ ppm $5.20(\mathrm{~d}, J=10 \mathrm{~Hz}, 1 \mathrm{H}$ ), 5.14 (hept, $J=6.3 \mathrm{~Hz}, 1 \mathrm{H}$ ), 4.18-4.12 (m, 1H), 4.10-3.95 (m, 3H), 2.98 (ddd, $J=$ 11.4, 8.6, $2.8 \mathrm{~Hz}, 1 \mathrm{H}$ ), 2.89 (dd, $J=13.8,11.1 \mathrm{~Hz}, 2 \mathrm{H}$ ), 2.54 (ddd, $J=13.8,4.0,0.8 \mathrm{~Hz}, 1 \mathrm{H}), 2.42-2.31(\mathrm{~m}, 1 \mathrm{H}$ ), 2.28-2.19 (m, 1H), $1.36(\mathrm{~d}, J=3.5 \mathrm{~Hz}, 3 \mathrm{H}), 1.34(\mathrm{~d}, J=3.5 \mathrm{~Hz}, 3 \mathrm{H}) .{ }^{13} \mathrm{C} \mathbf{N M R}\left(\mathbf{1 0 0} \mathbf{M H z}, \mathrm{CDCl}_{3}\right) \delta \mathrm{ppm} \mathrm{171.3,}$ 86.0, 71.2, 69.3, 66.9, 66.1, 49.5, 28.8, 27.3, 21.5, 21.0. HRMS (ESI+) $\mathrm{m} / \mathrm{z}$ calcd. for $[\mathrm{M}+\mathrm{Na}]^{+}:$285.0767; measured: 285.0769 .

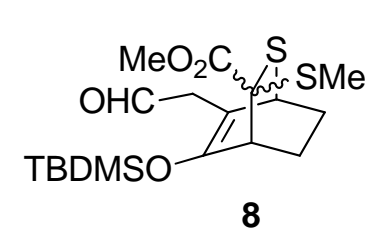

8

A mixture of the aldehyde $1 \mathrm{i}$ ( $0.5 \mathrm{mmol}, 1$ equiv), $\mathrm{PhCO}_{2} \mathrm{H}(0.1 \mathrm{mmol}, 0.2$ equiv) and catalyst 3 ( $0.1 \mathrm{mmol}, 0.2$ equiv) was dissolved in $1.5 \mathrm{~mL}$ of $\mathrm{CHCl}_{3}$ at rt. Then, the dithioester ( $1.0 \mathrm{mmol}, 2$ equiv) and water ( $2.5 \mathrm{mmol}, 5$ equiv) were added. The mixture was stirred for the time indicated. When the reaction was completed (20 h), the mixture was directly purified by FC on silica gel (pentane/EtOAc 10:1 to 4:1) to afford the corresponding cycloadduct $\mathbf{8}$ in $72 \%$ yield and $72: 28 \mathrm{dr}$ as a pale yellow oil. $[\boldsymbol{\alpha}]_{\mathbf{D}}^{\mathbf{2 0}}=-12.0$ (c 0.5 , $\left.\mathrm{CH}_{2} \mathrm{Cl}_{2}\right) .{ }^{1} \mathrm{H}$ NMR $\left(400 \mathrm{MHz}, \mathrm{CDCl}_{3}\right): \delta \mathrm{ppm} 9.70^{*}\left(\mathrm{t}, J=1.9 \mathrm{~Hz}, 1 \mathrm{H}^{*}\right), 9.62(\mathrm{~s}, 1 \mathrm{H}), 3.80^{*}\left(\mathrm{~s}, 3 \mathrm{H}^{*}\right), 3.70(\mathrm{~s}, 3 \mathrm{H})$, $3.58-3.09^{+}\left(\mathrm{m}, 3 \mathrm{H}, 4 \mathrm{H}^{*}\right), 2.61-2.46(\mathrm{~m}, 1 \mathrm{H}), 2.19-1.86^{+}\left(\mathrm{m}, 2 \mathrm{H}, 2 \mathrm{H}^{*}\right), 2.09(\mathrm{~s}, 3 \mathrm{H}), 2.02^{*}\left(\mathrm{~s}, 3 \mathrm{H}^{*}\right), 1.76-1.52^{+}$ $\left(\mathrm{m}, 2 \mathrm{H}, 2 \mathrm{H}^{*}\right), 0.95^{*}\left(\mathrm{~s}, 9 \mathrm{H}^{*}\right), 0.93(\mathrm{~s}, 9 \mathrm{H}), 0.21^{*}\left(\mathrm{~s}, 3 \mathrm{H}^{*}\right), 0.19^{*}\left(\mathrm{~s}, 3 \mathrm{H}^{*}\right), 0.14(\mathrm{~s}, 3 \mathrm{H}), 0.10(\mathrm{~s}, 3 \mathrm{H}) .{ }^{13} \mathrm{C}$ NMR (100 MHz, $\mathrm{CDCl}_{3}$ ): $\delta$ ppm 199.9, 199.7*, 171.4, 169.7*, 154.9, 150.7*, 110.4, 109.0*, 68.3*, 65.4, 53.0*, $52.8,45.7^{*}, 44.5,42.0^{*}, 41.7^{*}, 41.6,41.1,29.9,29.7^{*}, 25.6^{*}\left(3 C^{*}\right), 25.6$ (3C), 21.7*, 19.7, 18.0, 18.0*, 15.4, $14.2^{*},-3.5 *,-3.8,-3.9 *,-4.6$. HRMS (ESI+) $\mathrm{m} / z$ calcd. for $\mathrm{C}_{18} \mathrm{H}_{31} \mathrm{~S}_{2} \mathrm{O}_{4}[\mathrm{M}+\mathrm{H}]^{+}: 403.1428$; found: 403.1428. The ee was determined on HPLC after reduction to the corresponding alcohol $\left(\mathrm{NaBH}_{4}, \mathrm{MeOH}, 0{ }^{\circ} \mathrm{C}, 10 \mathrm{~min}\right)$; IC-5, $\mathrm{hx} / \mathrm{iPrOH}$ 90:10, $1.0 \mathrm{~mL} \cdot \mathrm{min}^{-1} ; \mathrm{t}_{\text {major }}=8.0 \mathrm{~min} ; \mathrm{t}_{\text {minor }}=9.4 \mathrm{~min}$ ( $96 \%$ ee) . 


\section{X-RAY STRUCTURE}

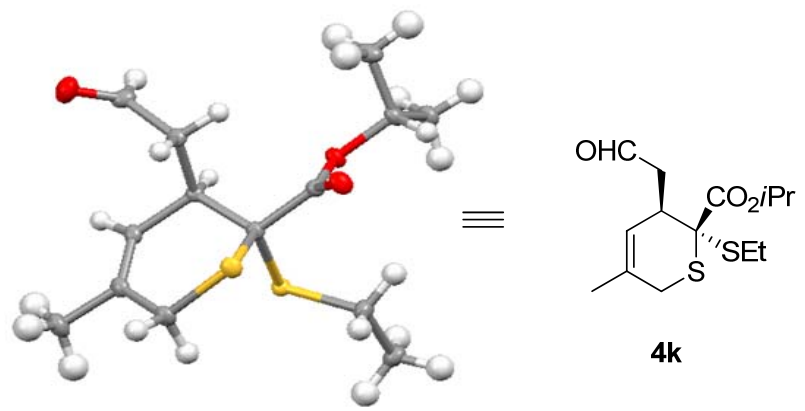

Crystal data for [4k]: $\mathrm{C}_{14} \mathrm{H}_{22} \mathrm{O}_{3} \mathrm{~S}_{2}, M=302.44$, monoclinic, space group P 1211 (no. 6), $a=9.2144$ (0) $\AA, b=$ 9.3239(0) $\AA, c=9.5262(0) \AA, b=109.663(0)^{\circ}, V=770.71(0) \AA^{3}, T=0 \mathrm{~K}, Z=2, \mathrm{~d}_{\mathrm{c}}=1.303 \mathrm{~g} \mathrm{~cm}^{-3}, \mu($ Mo Ka, $\lambda=$ $0.71073 \AA)=0.347 \mathrm{~mm}^{-1}, 16358$ reflections collected, 5455 unique $\left[R_{\text {int }}=0.026\right]$, which were used in all calculations. Refinement on $F^{2}$, final $R(F)=0.0308, R_{w}(F 2)=0$. CCDC number 909665 . 


\section{COMPUTATIONAL DATA}

\subsection{Methods}

For studying the distinct reaction pathways: All calculations were run using GAUSSIAN09. ${ }^{4}$ All calculations were performed at wB97xd/tzvp level of theory and basis set. ${ }^{5}$ Solvent effects were estimated with IEFPCM, using chloroform as the solvent. Frequency calculations were performed for all stationary points to identify them as local minima or first order saddle points and to obtain the ZPEs and thermochemical corrections for the free energies. All of the reported values are free energies at $298.15 \mathrm{~K}$ and $1 \mathrm{~atm}$. Intrinsic reaction coordinate (IRC) calculations were used to characterize transition state structures. ${ }^{6}$ Structural images were created using Chemcraft. ${ }^{7}$

For studying the origin of enantioselectivity and the facial shielding provided by catalyst 3: All structures were optimized in solvent (IEFPCM, $\mathrm{CHCl}_{3}$ ) with wB97xd/6-31G(d). The activation energies reported in the manuscript were obtained with wB97xd/cc-pVDZ single points. Frequency calculations were performed for all stationary points to identify them as local minima or first order saddle points and to obtain the ZPEs and thermochemical corrections for the free energies. All of the reported values are free energies at $298.15 \mathrm{~K}$ and $1 \mathrm{~atm}$. Structures were generated with Chemcraft.

\subsection{Energies and coordinates: path I-II}

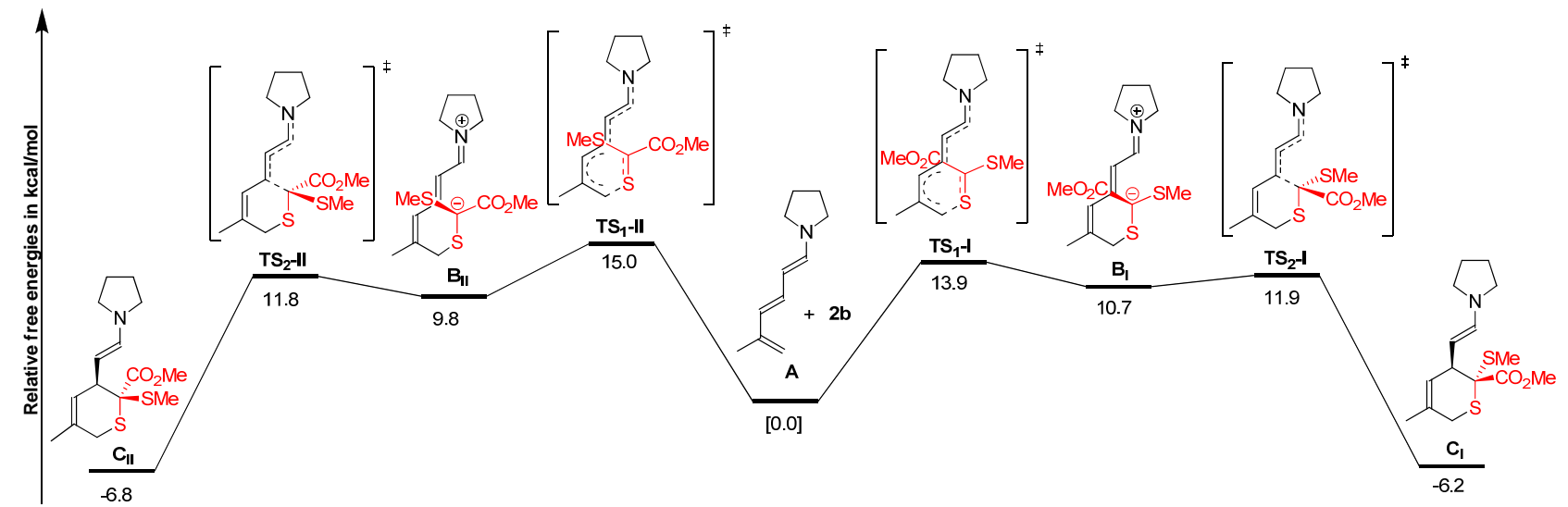

Figure S1: Energy profiles for pathways I-II: the formal cycloaddition of $\mathbf{A}$ and $\mathbf{2 b}$ (initial attack at sulfur). Reported values in plain text are wB97xd/tzvp free energies in solvent (IEFPCM, $\left.\mathrm{CHCl}_{3}\right)$, relative to $\mathbf{A}+\mathbf{2 b}$.

\footnotetext{
${ }^{4}$ S1. GAUSSIAN09, Revision A.02 Frisch, M. J.; Trucks, G. W.; Schlegel, H. B.; Scuseria, G. E.; Robb, M. A.; Cheeseman, J. R.; Scalmani, G.; Barone, V.; Mennucci, B.; Petersson, G. A.; Nakatsuji, H.; Caricato, M.; Li, X.; Hratchian, H. P.; Izmaylov, A. F.; Bloino, J.; Zheng, G.; Sonnenberg, J. L.; Hada, M.; Ehara, M.; Toyota, K.; Fukuda, R.; Hasegawa, J.; Ishida, M.; Nakajima, T.; Honda, Y.; Kitao, O.; Nakai, H.; Vreven, T.; Montgomery, Jr., J. A.; Peralta, J. E.; Ogliaro, F.; Bearpark, M.; Heyd, J. J.; Brothers, E.; Kudin, K. N.; Staroverov, V. N.; Kobayashi, R.; Normand, J.; Raghavachari, K.; Rendell, A.; Burant, J. C.; Iyengar, S. S.; Tomasi, J.; Cossi, M.; Rega, N.; Millam, N. J.; Klene, M.; Knox, J. D.; Cross, J. B.; Bakken, V.; Adamo, C.; Jaramillo, J.; Gomperts, R.; Stratmann, R. E.; Yazyev, O.; Austin, A. J.; Cammi, R.; Pomelli, C.; Ochterski, J. W.; Martin, R. L.; Morokuma, K.; Zakrzewski, V. G.; Voth, G. A.; Salvador, P.; Dannenberg, J. J.; Dapprich, S.; Daniels, A. D.; Farkas, Ö.; Foresman, J. B.; Ortiz, J. V.; Cioslowski, J.; Fox, D. J., Gaussian, Inc., Wallingford CT, 2009.
}

${ }^{5}$ (a) Chai, J.-D.; Head-Gordon, M. J. Chem. Phys. 2008, 10, 6615. (b) Schaefer, A.; Huber, C.; Ahlrichs, R. J. Chem. Phys. 1994, 100, 5829.

${ }^{6}$ (a) Gonzalez, C.; Schlegel, H. B. J. Phys. Chem. 1990, 94, 5523. (b) Fukui, K. Acc. Chem. Res. 1981, 14, 363.

${ }^{7}$ Zhurko, G. A. Chemcraft, Version 1.7 (build 365). 
A

$\mathrm{HF}=-484.1123161$ hartrees

Imaginary Frequencies: none found

Zero-point correction $=0.259331$ (Hartree/Particle)

Temperature: 298.150 Kelvin. Pressure: 1.00000 Atm.

Sum of electronic and thermal Free Energies $=-483.893431$ hartrees

Coordinates:

\begin{tabular}{lcccc} 
Center & Atomic & \multicolumn{3}{c}{ Coordinates (Angstroms) } \\
Number & Number & $X$ & Y & Z
\end{tabular}

$\begin{array}{ccccc}1 & 6 & 5.078540 & -0.961156 & 0.448109 \\ 2 & 1 & 4.823507 & -1.325694 & 1.446707 \\ 3 & 1 & 6.077182 & -0.525350 & 0.481180 \\ 4 & 1 & 5.105222 & -1.832965 & -0.212010 \\ 5 & 6 & 4.053631 & 0.031447 & -0.036216 \\ 6 & 6 & 2.679299 & -0.480418 & -0.114965 \\ 7 & 1 & 2.577710 & -1.557781 & -0.229588 \\ 8 & 6 & 1.563237 & 0.260918 & -0.015766 \\ 9 & 1 & 1.672276 & 1.330814 & 0.157220 \\ 10 & 6 & 0.212547 & -0.230000 & -0.100266 \\ 11 & 1 & 0.062798 & -1.290122 & -0.275469 \\ 12 & 6 & -0.852035 & 0.598001 & 0.036209 \\ 13 & 1 & -0.678745 & 1.657105 & 0.207447 \\ 14 & 6 & -2.614444 & -1.108943 & -0.208476 \\ 15 & 6 & -3.251043 & 1.204116 & 0.130401 \\ 16 & 6 & -4.134091 & -0.968922 & -0.323344 \\ 17 & 1 & -2.167803 & -1.548084 & -1.105701 \\ 18 & 1 & -2.322966 & -1.732732 & 0.645536 \\ 19 & 6 & -4.438374 & 0.306630 & 0.467254 \\ 20 & 1 & -3.032464 & 1.933999 & 0.911582 \\ 21 & 1 & -3.425042 & 1.745999 & -0.807047 \\ 22 & 1 & -4.658421 & -1.844105 & 0.057046 \\ 23 & 1 & -4.415959 & -0.833965 & -1.369684 \\ 24 & 1 & -4.450001 & 0.093978 & 1.538472 \\ 25 & 1 & -5.391772 & 0.759137 & 0.198361 \\ 26 & 7 & -2.154007 & 0.255053 & -0.012169 \\ 27 & 6 & 4.402055 & 1.277590 & -0.371352 \\ 28 & 1 & 3.683464 & 1.985413 & -0.767972 \\ 29 & 1 & 5.423138 & 1.623409 & -0.260419\end{array}$


$\mathrm{HF}=-1102.8699914$ hartrees

Imaginary Frequencies: none found

Zero-point correction $=0.099701$ (Hartree/Particle)

Temperature: 298.150 Kelvin. Pressure: 1.00000 Atm.

Sum of electronic and thermal Free Energies $=-1102.806393$ hartrees

Coordinates:

\begin{tabular}{|c|c|c|c|c|}
\hline \multirow{2}{*}{$\begin{array}{l}\text { Center } \\
\text { Number }\end{array}$} & \multicolumn{2}{|c|}{ Atomic } & \multicolumn{2}{|c|}{ Coordinates (Angstroms) } \\
\hline & & & $x$ & Z \\
\hline 1 & 6 & -3.345812 & -0.014575 & -0.304450 \\
\hline 2 & 1 & -3.924343 & 0.758886 & -0.799262 \\
\hline 3 & 1 & -3.655998 & -0.120208 & 0.734323 \\
\hline 4 & 1 & -3.458926 & -0.967863 & -0.819243 \\
\hline 5 & 8 & -1.982581 & 0.428343 & -0.369710 \\
\hline 6 & 6 & -1.082841 & -0.378512 & 0.156252 \\
\hline 7 & 8 & -1.317198 & -1.450105 & 0.653372 \\
\hline 8 & 6 & 0.326568 & 0.191505 & 0.068763 \\
\hline 9 & 16 & 0.638704 & 1.789284 & 0.213765 \\
\hline 10 & 16 & 1.470064 & $4-1.094042$ & -0.144785 \\
\hline 11 & 6 & 3.041389 & -0.209088 & -0.218426 \\
\hline 12 & 1 & 3.224744 & 0.306675 & 0.721993 \\
\hline 13 & 1 & 3.800644 & -0.970552 & -0.385883 \\
\hline 14 & 1 & 3.036001 & 0.507296 & -1.037744 \\
\hline
\end{tabular}


$\mathrm{TS}_{1}-\mathrm{I}$

$\mathrm{HF}=-1586.984029$ hartrees

Imaginary Frequencies: 1 (-271.8276 1/cm)

Zero-point correction $=0.360525$ (Hartree/Particle)

Temperature: 298.150 Kelvin. Pressure: 1.00000 Atm.

Sum of electronic and thermal Free Energies $=-1586.677598$ hartrees

Coordinates:

\begin{tabular}{|c|c|c|c|c|}
\hline \multirow{2}{*}{$\begin{array}{l}\text { Center } \\
\text { Number }\end{array}$} & Atomic & & \multicolumn{2}{|c|}{ Coordinates (Angstroms) } \\
\hline & Numbe & & $X \quad Y$ & Z \\
\hline 1 & 6 & 3.708521 & -2.543421 & -0.993773 \\
\hline 2 & 1 & 3.359498 & -3.512242 & -1.354545 \\
\hline 3 & 1 & 3.787468 & -2.607268 & 0.095820 \\
\hline 4 & 1 & 4.705971 & -2.361380 & -1.393046 \\
\hline 5 & 6 & 2.745962 & -1.449929 & -1.370164 \\
\hline 6 & 6 & 1.373610 & -1.733163 & -1.263529 \\
\hline 7 & 1 & 1.102223 & -2.744720 & -0.972942 \\
\hline 8 & 6 & 0.356927 & -0.812258 & -1.383085 \\
\hline 9 & 1 & 0.600674 & 0.198273 & -1.693135 \\
\hline 10 & 6 & -0.998049 & $9-1.070020$ & -1.119688 \\
\hline 11 & 1 & -1.291141 & $1-2.056417$ & -0.779063 \\
\hline 12 & 6 & -1.914987 & $7-0.048977$ & -1.162461 \\
\hline 13 & 1 & -1.589894 & 0.930995 & -1.497742 \\
\hline 14 & 6 & -3.779362 & $2-1.313124$ & -0.189612 \\
\hline 15 & 6 & -4.101998 & 31.018142 & -0.783859 \\
\hline 16 & 6 & -5.226870 & -0.903852 & 0.081678 \\
\hline 17 & 1 & -3.700833 & $3-2.172542$ & -0.859048 \\
\hline 18 & 1 & -3.240484 & $4-1.548705$ & 0.734343 \\
\hline 19 & 6 & -5.140147 & $7 \quad 0.614369$ & 0.257549 \\
\hline 20 & 1 & -3.562262 & 21.929509 & -0.527095 \\
\hline 21 & 1 & -4.562339 & $\begin{array}{ll}9 & 1.148637\end{array}$ & -1.768375 \\
\hline 22 & 1 & -5.638443 & $3-1.411398$ & 0.952187 \\
\hline 23 & 1 & -5.852314 & -1.147101 & -0.779763 \\
\hline 24 & 1 & -4.774421 & 0.857744 & 1.256876 \\
\hline 25 & 1 & -6.094363 & 1.116692 & 0.107680 \\
\hline 26 & 7 & -3.188558 & -0.125425 & -0.799642 \\
\hline 27 & 6 & 3.240416 & -0.176916 & -1.627281 \\
\hline 28 & 1 & 2.620728 & 0.562174 & -2.117144 \\
\hline 29 & 1 & 4.303921 & -0.056457 & -1.794844 \\
\hline 30 & 6 & 0.601408 & -1.974081 & 2.717646 \\
\hline
\end{tabular}




$\begin{array}{lcccc}31 & 1 & 1.298799 & -2.751661 & 3.018251 \\ 32 & 1 & -0.164416 & -2.389696 & 2.060521 \\ 33 & 1 & 0.121761 & -1.540520 & 3.595646 \\ 34 & 8 & 1.380551 & -1.004821 & 2.027077 \\ 35 & 6 & 0.706798 & 0.058783 & 1.560713 \\ 36 & 8 & -0.472634 & 0.238873 & 1.801266 \\ 37 & 6 & 1.514609 & 0.987816 & 0.763490 \\ 38 & 16 & 3.126209 & 0.784685 & 0.443264 \\ 39 & 16 & 0.526126 & 2.385242 & 0.229807 \\ 40 & 6 & 1.774403 & 3.469275 & -0.506968 \\ 41 & 1 & 1.234601 & 4.355732 & -0.838141 \\ 42 & 1 & 2.524550 & 3.750477 & 0.230302 \\ 43 & 1 & 2.262486 & 3.000739 & -1.359064\end{array}$

IRC path for $\mathrm{TS}_{1}-$ I:

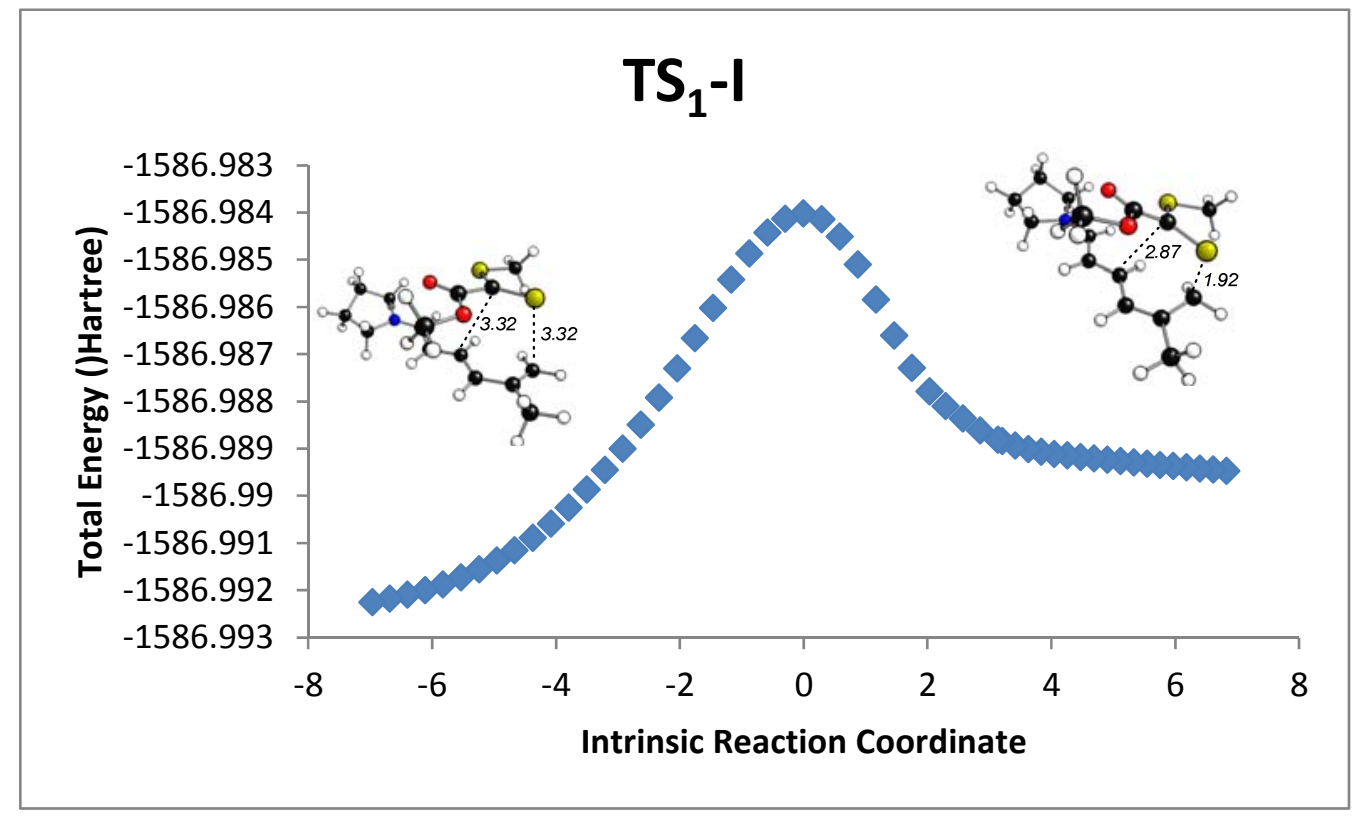


$\mathrm{TS}_{1}-\mathrm{II}$

$\mathrm{HF}=-1586.9825849$ hartrees

Imaginary Frequencies: 1 (-288.6569 1/cm)

Zero-point correction $=0.360557$ (Hartree/Particle)

Temperature: 298.150 Kelvin. Pressure: 1.00000 Atm.

Sum of electronic and thermal Free Energies $=-1586.675982$ hartrees

Coordinates:

\begin{tabular}{|c|c|c|c|c|}
\hline \multirow{2}{*}{$\begin{array}{l}\text { Center } \\
\text { Number }\end{array}$} & \multicolumn{2}{|c|}{ Atomic } & \multicolumn{2}{|c|}{ Coordinates (Angstroms) } \\
\hline & & & $\mathrm{Y}$ & Z \\
\hline 1 & 6 & 3.911580 & -2.320274 & -1.133468 \\
\hline 2 & 1 & 4.007079 & -2.493925 & -0.056377 \\
\hline 3 & 1 & 4.887092 & -2.009823 & -1.506714 \\
\hline 4 & 1 & 3.649627 & -3.274271 & -1.593769 \\
\hline 5 & 6 & 2.856018 & -1.283402 & -1.405075 \\
\hline 6 & 6 & 1.511684 & -1.670889 & -1.264062 \\
\hline 7 & 1 & 1.324754 & -2.702300 & -0.975724 \\
\hline 8 & 6 & 0.421969 & -0.832493 & -1.352711 \\
\hline 9 & 1 & 0.571702 & 0.178309 & -1.720624 \\
\hline 10 & 6 & -0.891849 & -1.195061 & -1.008845 \\
\hline 11 & 1 & -1.076544 & -2.190425 & -0.622639 \\
\hline 12 & 6 & -1.901107 & $7-0.266094$ & -1.038573 \\
\hline 13 & 1 & -1.687936 & 0.728298 & -1.415423 \\
\hline 14 & 6 & -3.629154 & -1.672899 & -0.005521 \\
\hline 15 & 6 & -4.140752 & 0.632329 & -0.573584 \\
\hline 16 & 6 & -5.102075 & -1.384007 & 0.280750 \\
\hline 17 & 1 & -3.484232 & -2.528041 & L -0.668920 \\
\hline 18 & 1 & -3.062876 & -1.854577 & $7 \quad 0.914702$ \\
\hline 19 & 6 & -5.133350 & 0.134280 & 0.472550 \\
\hline 20 & 1 & -3.657489 & 1.569423 & -0.297002 \\
\hline 21 & 1 & -4.623557 & 0.757961 & -1.547795 \\
\hline 22 & 1 & -5.464543 & -1.931625 & 1.148848 \\
\hline 23 & 1 & -5.712772 & -1.667594 & -0.579037 \\
\hline 24 & 1 & -4.778293 & 0.394398 & 1.471537 \\
\hline 25 & 1 & -6.124937 & 0.563028 & 0.337571 \\
\hline 26 & 7 & -3.152004 & -0.445517 & $7-0.634368$ \\
\hline 27 & 6 & 3.236604 & 0.037877 & -1.594773 \\
\hline 28 & 1 & 2.546876 & 0.758113 & -2.012529 \\
\hline 29 & 1 & 4.283226 & 0.255483 & -1.771855 \\
\hline 30 & 6 & -0.061892 & 3.490994 & -1.008409 \\
\hline
\end{tabular}




$\begin{array}{lcccc}31 & 1 & 0.451317 & 4.038774 & -1.794146 \\ 32 & 1 & -0.356773 & 4.169277 & -0.207541 \\ 33 & 1 & -0.954067 & 3.010365 & -1.412321 \\ 34 & 8 & 0.874179 & 2.525758 & -0.538455 \\ 35 & 6 & 0.455279 & 1.737541 & 0.468720 \\ 36 & 8 & -0.679543 & 1.795054 & 0.903908 \\ 37 & 6 & 1.471977 & 0.801011 & 0.951594 \\ 38 & 16 & 3.069544 & 0.901567 & 0.513481 \\ 39 & 16 & 0.781011 & -0.314278 & 2.170338 \\ 40 & 6 & 2.150655 & -1.460289 & 2.440077 \\ 41 & 1 & 1.794563 & -2.179895 & 3.176333 \\ 42 & 1 & 2.400547 & -1.978178 & 1.514344 \\ 43 & 1 & 3.031745 & -0.949042 & 2.822921\end{array}$

IRC path for $\mathrm{TS}_{1}-$ II:

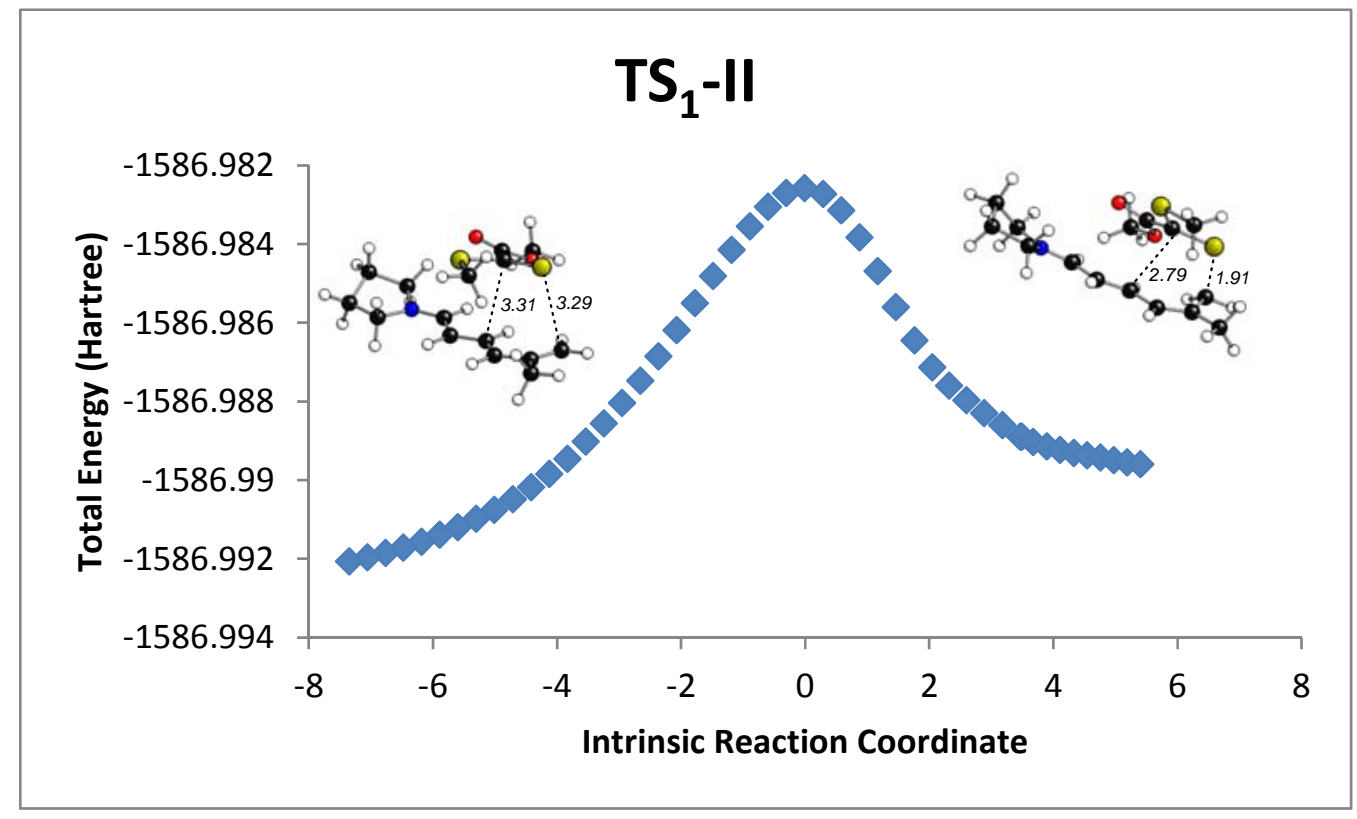


$\mathbf{B}_{1}$

$\mathrm{HF}=-1586.9934003$ hartrees

Imaginary Frequencies: none found

Zero-point correction $=0.363024$ (Hartree/Particle)

Temperature: 298.150 Kelvin. Pressure: 1.00000 Atm.

Sum of electronic and thermal Free Energies $=-1586.682742$ hartrees

Coordinates:

\begin{tabular}{|c|c|c|c|c|}
\hline \multirow{2}{*}{$\begin{array}{l}\text { Center } \\
\text { Number }\end{array}$} & \multicolumn{2}{|c|}{ Atomic } & \multicolumn{2}{|c|}{ Coordinates (Angstroms) } \\
\hline & & & $x$ & Z \\
\hline 1 & 6 & 4.155360 & -2.249403 & -0.328216 \\
\hline 2 & 1 & 3.812340 & -3.239570 & -0.030244 \\
\hline 3 & 1 & 4.593389 & -1.754911 & 0.543111 \\
\hline 4 & 1 & 4.952019 & -2.361551 & -1.068697 \\
\hline 5 & 6 & 3.043687 & -1.412519 & -0.881178 \\
\hline 6 & 6 & 1.780753 & -1.898650 & -0.947442 \\
\hline 7 & 1 & 1.591282 & -2.894770 & -0.558342 \\
\hline 8 & 6 & 0.647002 & -1.143379 & -1.357770 \\
\hline 9 & 1 & 0.808940 & -0.250262 & -1.948992 \\
\hline 10 & 6 & -0.647442 & -1.486763 & -1.064609 \\
\hline 11 & 1 & -0.851468 & $3-2.353524$ & -0.448539 \\
\hline 12 & 6 & -1.668264 & -0.579340 & -1.362949 \\
\hline 13 & 1 & -1.439412 & 0.268673 & -1.999187 \\
\hline 14 & 6 & -3.317699 & -1.558296 & 0.150710 \\
\hline 15 & 6 & -3.880740 & 0.428973 & -1.129797 \\
\hline 16 & 6 & -4.736283 & $3-1.101864$ & 0.488597 \\
\hline 17 & 1 & -3.269895 & -2.587312 & -0.206631 \\
\hline 18 & 1 & -2.633382 & - -1.437795 & 0.994915 \\
\hline 19 & 6 & -4.723480 & 0.388257 & 0.138692 \\
\hline 20 & 1 & -3.377764 & 1.380207 & -1.296271 \\
\hline 21 & 1 & -4.476730 & 0.179105 & -2.011283 \\
\hline 22 & 1 & -4.980195 & $5-1.291602$ & 1.531955 \\
\hline 23 & 1 & -5.462020 & -1.630129 & -0.132775 \\
\hline 24 & 1 & -4.227617 & 0.957021 & 0.927090 \\
\hline 25 & 1 & -5.718618 & 0.801078 & -0.015636 \\
\hline 26 & 7 & -2.885764 & -0.625845 & -0.898393 \\
\hline 27 & 6 & 3.373930 & -0.009035 & -1.186617 \\
\hline 28 & 1 & 2.825512 & 0.417753 & -2.023345 \\
\hline 29 & 1 & 4.440741 & 0.132652 & -1.353784 \\
\hline 30 & 6 & 0.376449 & -1.230426 & 2.95414 \\
\hline
\end{tabular}




$\begin{array}{lcccc}31 & 1 & 1.088040 & -1.813047 & 3.536059 \\ 32 & 1 & -0.284485 & -1.902587 & 2.402043 \\ 33 & 1 & -0.230881 & -0.615617 & 3.620874 \\ 34 & 8 & 1.152172 & -0.436454 & 2.074758 \\ 35 & 6 & 0.446067 & 0.394591 & 1.249699 \\ 36 & 8 & -0.779829 & 0.454183 & 1.349137 \\ 37 & 6 & 1.242848 & 1.120737 & 0.321255 \\ 38 & 16 & 2.980596 & 1.063522 & 0.319715 \\ 39 & 16 & 0.416995 & 2.238499 & -0.774010 \\ 40 & 6 & -0.061157 & 3.622123 & 0.314189 \\ 41 & 1 & -0.583831 & 4.357894 & -0.297804 \\ 42 & 1 & -0.726552 & 3.257396 & 1.094719 \\ 43 & 1 & 0.824550 & 4.077624 & 0.754844\end{array}$


$B_{\text {II }}$

$H F=-1586.9939022$ hartrees

Imaginary Frequencies: none found

Zero-point correction $=0.362850$ (Hartree/Particle)

Temperature: 298.150 Kelvin. Pressure: 1.00000 Atm.

Sum of electronic and thermal Free Energies $=-1586.684210$ hartrees

Coordinates:

\begin{tabular}{cccccc} 
Center & \multicolumn{2}{c}{ Atomic } & \multicolumn{3}{c}{ Coordinates (Angstroms) } \\
Number & Number & X & $Y$ & $Z$ \\
-------------------------------- \\
1 & 6 & -3.982629 & 2.694517 & -0.057840 \\
2 & 1 & -4.545778 & 2.155128 & 0.708350 \\
3 & 1 & -4.689903 & 2.964117 & -0.846946 \\
4 & 1 & -3.585022 & 3.608172 & 0.382444 \\
5 & 6 & -2.898104 & 1.818925 & -0.605478 \\
6 & 6 & -1.595810 & 2.186308 & -0.514199 \\
7 & 1 & -1.352831 & 3.115364 & -0.007263 \\
8 & 6 & -0.505032 & 1.381771 & -0.945471 \\
9 & 1 & -0.709335 & 0.570163 & -1.633659 \\
10 & 6 & 0.801585 & 1.604408 & -0.587530 \\
11 & 1 & 1.043955 & 2.394556 & 0.111931 \\
12 & 6 & 1.784174 & 0.697385 & -0.989171 \\
13 & 1 & 1.521008 & -0.082393 & -1.692914 \\
14 & 6 & 3.559155 & 1.576556 & 0.458359 \\
15 & 6 & 3.979384 & -0.372882 & -0.936964 \\
16 & 6 & 5.018194 & 1.144393 & 0.589421 \\
17 & 1 & 3.443319 & 2.617519 & 0.155527 \\
18 & 1 & 3.001209 & 1.418781 & 1.385323 \\
19 & 6 & 4.984869 & -0.337230 & 0.207914 \\
20 & 1 & 3.455727 & -1.322568 & -1.034087 \\
21 & 1 & 4.452724 & -0.121593 & -1.889508 \\
22 & 1 & 5.403051 & 1.318534 & 1.592217 \\
23 & 1 & 5.639042 & 1.702174 & -0.114337 \\
24 & 1 & 4.615268 & -0.934887 & 1.042819 \\
25 & 1 & 5.957657 & -0.724394 & -0.089134 \\
26 & 7 & 3.021973 & 0.680135 & -0.571714 \\
27 & 6 & -3.304235 & 0.491731 & -1.095938 \\
28 & 1 & -2.719145 & 0.105857 & -1.926616 \\
29 & 1 & -4.362237 & 0.452794 & -1.350986 \\
30 & 6 & -0.341659 & -2.788252 & -2.284931 \\
& & & &
\end{tabular}




$\begin{array}{lcccc}31 & 1 & -0.988781 & -3.070146 & -3.112979 \\ 32 & 1 & 0.020446 & -3.683575 & -1.777207 \\ 33 & 1 & 0.518779 & -2.233574 & -2.665834 \\ 34 & 8 & -1.139286 & -1.992553 & -1.424362 \\ 35 & 6 & -0.541404 & -1.578164 & -0.259116 \\ 36 & 8 & 0.654779 & -1.806722 & -0.077777 \\ 37 & 6 & -1.407198 & -0.846241 & 0.595070 \\ 38 & 16 & -3.122704 & -0.754525 & 0.311372 \\ 39 & 16 & -0.736147 & -0.177177 & 2.087249 \\ 40 & 6 & -0.565465 & -1.655474 & 3.144858 \\ 41 & 1 & -0.143440 & -1.330407 & 4.096550 \\ 42 & 1 & -1.536855 & -2.116854 & 3.318498 \\ 43 & 1 & 0.110037 & -2.368784 & 2.675088\end{array}$


$\mathrm{TS}_{2}-\mathrm{I}$

$\mathrm{HF}=-1586.990859$ hartrees

Imaginary Frequencies: 1 (-206.2525 1/cm)

Zero-point correction $=0.362215$ (Hartree/Particle)

Temperature: 298.150 Kelvin. Pressure: 1.00000 Atm.

Sum of electronic and thermal Free Energies $=-1586.680794$ hartrees

Coordinates:

\begin{tabular}{cccccc} 
Center & \multicolumn{2}{c}{ Atomic } & \multicolumn{3}{c}{ Coordinates (Angstroms) } \\
Number & Number & X & $Y$ & $Z$ \\
----------------------------------- \\
1 & 6 & 4.019717 & -2.503327 & -0.592773 \\
2 & 1 & 3.610607 & -3.444224 & -0.225275 \\
3 & 1 & 4.624431 & -2.055623 & 0.201487 \\
4 & 1 & 4.691643 & -2.716240 & -1.428335 \\
5 & 6 & 2.938731 & -1.553022 & -1.005163 \\
6 & 6 & 1.633256 & -1.850208 & -0.849236 \\
7 & 1 & 1.363123 & -2.798136 & -0.393567 \\
8 & 6 & 0.558631 & -0.941854 & -1.134985 \\
9 & 1 & 0.695172 & -0.238893 & -1.948507 \\
10 & 6 & -0.762084 & -1.243236 & -0.762450 \\
11 & 1 & -0.926160 & -2.001768 & -0.006823 \\
12 & 6 & -1.813592 & -0.481921 & -1.212793 \\
13 & 1 & -1.630774 & 0.270046 & -1.973182 \\
14 & 6 & -3.476273 & -1.372785 & 0.350398 \\
15 & 6 & -4.138947 & 0.320459 & -1.258390 \\
16 & 6 & -4.963803 & -1.058204 & 0.508982 \\
17 & 1 & -3.286549 & -2.431331 & 0.161438 \\
18 & 1 & -2.891454 & -1.067966 & 1.224565 \\
19 & 6 & -5.096505 & 0.352083 & -0.072079 \\
20 & 1 & -3.736370 & 1.298635 & -1.519891 \\
21 & 1 & -4.619959 & -0.109919 & -2.141969 \\
22 & 1 & -5.285145 & -1.124991 & 1.546788 \\
23 & 1 & -5.560640 & -1.760743 & -0.076329 \\
24 & 1 & -4.759969 & 1.093871 & 0.654525 \\
25 & 1 & -6.115212 & 0.596521 & -0.368485 \\
26 & 7 & -3.065098 & -0.558480 & -0.791421 \\
27 & 6 & 3.349184 & -0.192225 & -1.399273 \\
28 & 1 & 2.799043 & 0.228073 & -2.239267 \\
29 & 1 & 4.416579 & -0.112062 & -1.597153 \\
30 & 6 & 0.741497 & -0.964085 & 3.235588
\end{tabular}




$\begin{array}{lcccc}31 & 1 & 1.487103 & -1.602080 & 3.704324 \\ 32 & 1 & -0.127606 & -1.557500 & 2.946857 \\ 33 & 1 & 0.422149 & -0.189239 & 3.934101 \\ 34 & 8 & 1.378145 & -0.400645 & 2.097274 \\ 35 & 6 & 0.617528 & 0.433043 & 1.347759 \\ 36 & 8 & -0.535336 & 0.680304 & 1.658812 \\ 37 & 6 & 1.278961 & 0.900632 & 0.143793 \\ 38 & 16 & 3.035993 & 0.921211 & 0.078502 \\ 39 & 16 & 0.416245 & 2.137211 & -0.806229 \\ 40 & 6 & 0.548022 & 3.610838 & 0.254352 \\ 41 & 1 & 0.070555 & 4.437031 & -0.272080 \\ 42 & 1 & 0.034974 & 3.435669 & 1.198401 \\ 43 & 1 & 1.595939 & 3.851073 & 0.430887\end{array}$

IRC path for $\mathrm{TS}_{2}-$ I:

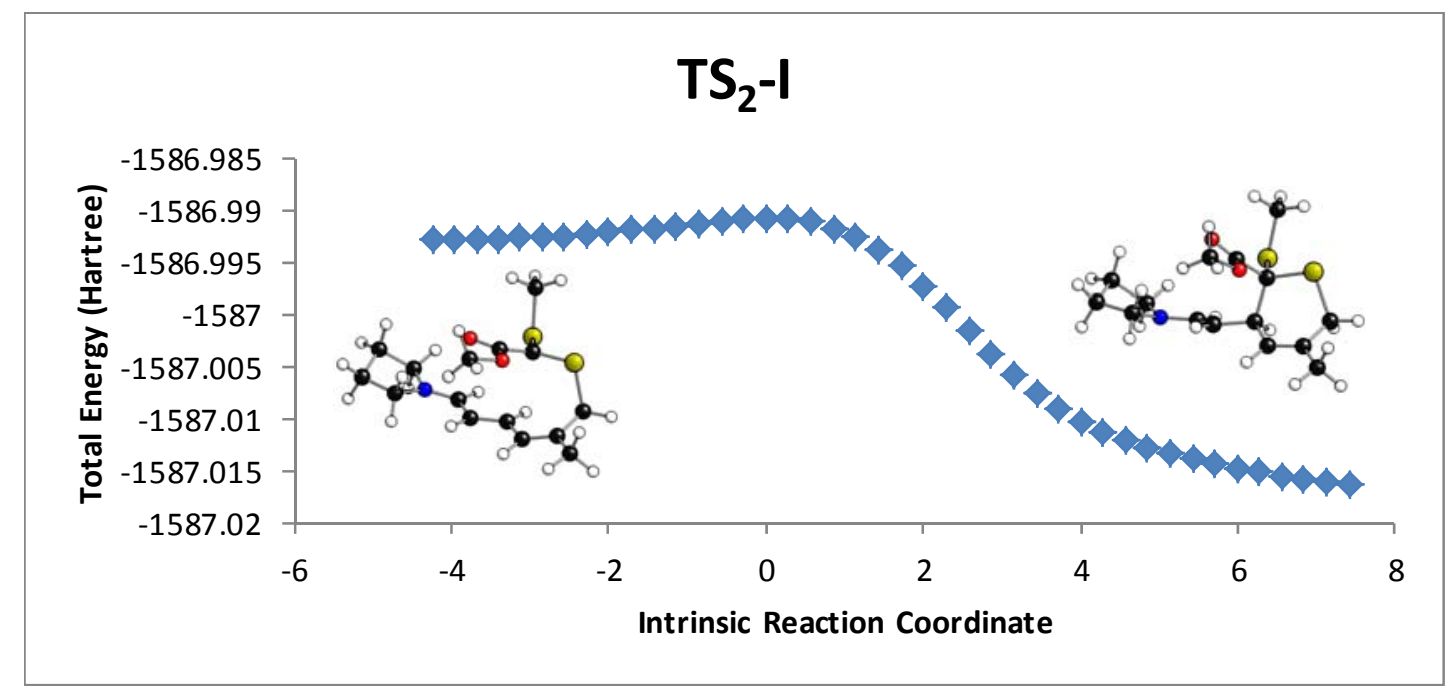


$\mathrm{TS}_{2}-\mathrm{II}$

$\mathrm{HF}=-1586.9911468$ hartrees

Imaginary Frequencies: 1 (-227.7800 1/cm)

Zero-point correction $=0.362659$ (Hartree/Particle)

Temperature: 298.150 Kelvin. Pressure: 1.00000 Atm.

Sum of electronic and thermal Free Energies $=-1586.680975$ hartrees

Coordinates:

\begin{tabular}{|c|c|c|c|c|}
\hline \multirow{2}{*}{$\begin{array}{l}\text { Center } \\
\text { Number }\end{array}$} & \multicolumn{2}{|c|}{ Atomic } & \multicolumn{2}{|c|}{ Coordinates (Angstroms) } \\
\hline & & & $x$ & Z \\
\hline 1 & 6 & 4.005806 & -2.770253 & -0.287409 \\
\hline 2 & 1 & 4.666828 & -2.286392 & 0.437359 \\
\hline 3 & 1 & 4.620636 & -3.052750 & -1.146214 \\
\hline 4 & 1 & 3.601836 & -3.675686 & 0.164864 \\
\hline 5 & 6 & 2.919964 & -1.825581 & -0.699109 \\
\hline 6 & 6 & 1.618123 & -2.081251 & -0.459718 \\
\hline 7 & 1 & 1.342586 & -2.993504 & 0.060410 \\
\hline 8 & 6 & 0.561917 & -1.157066 & -0.768336 \\
\hline 9 & 1 & 0.714874 & -0.494489 & -1.611975 \\
\hline 10 & 6 & -0.768549 & -1.410544 & -0.392090 \\
\hline 11 & 1 & -0.963747 & -2.150252 & 0.374346 \\
\hline 12 & 6 & -1.793195 & -0.642385 & -0.889410 \\
\hline 13 & 1 & -1.575067 & 0.090898 & -1.658276 \\
\hline 14 & 6 & -3.548676 & $5-1.527734$ & 0.578666 \\
\hline 15 & 6 & -4.092309 & 0.214234 & -1.024287 \\
\hline 16 & 6 & -5.040459 & -1.202503 & 0.649874 \\
\hline 17 & 1 & -3.354294 & -2.584541 & 0.385130 \\
\hline 18 & 1 & -3.022053 & -1.246062 & 1.496322 \\
\hline 19 & 6 & -5.121537 & 0.224301 & 0.101214 \\
\hline 20 & 1 & -3.655815 & 1.193351 & -1.220610 \\
\hline 21 & 1 & -4.525515 & -0.173116 & -1.951553 \\
\hline 22 & 1 & -5.430195 & -1.294579 & 1.662016 \\
\hline 23 & 1 & -5.603631 & -1.882795 & 0.007673 \\
\hline 24 & 1 & -4.823221 & 0.942230 & 0.867373 \\
\hline 25 & 1 & -6.116772 & 0.489853 & -0.251416 \\
\hline 26 & 7 & -3.064789 & -0.701342 & -0.524800 \\
\hline 27 & 6 & 3.324531 & -0.504431 & -1.216491 \\
\hline 28 & 1 & 2.747883 & -0.150685 & -2.068568 \\
\hline 29 & 1 & 4.385253 & -0.451794 & -1.455829 \\
\hline 30 & 6 & 0.125608 & 2.855031 & -2.162017 \\
\hline
\end{tabular}




$\begin{array}{lcccc}31 & 1 & 0.663038 & 3.056914 & -3.085280 \\ 32 & 1 & -0.043172 & 3.785095 & -1.617970 \\ 33 & 1 & -0.840093 & 2.399057 & -2.385986 \\ 34 & 8 & 0.953750 & 1.971021 & -1.417016 \\ 35 & 6 & 0.469561 & 1.573427 & -0.210896 \\ 36 & 8 & -0.645741 & 1.904826 & 0.152910 \\ 37 & 6 & 1.353824 & 0.653547 & 0.477477 \\ 38 & 16 & 3.087448 & 0.741488 & 0.167961 \\ 39 & 16 & 0.849916 & 0.136470 & 2.103286 \\ 40 & 6 & 1.004617 & 1.673096 & 3.071203 \\ 41 & 1 & 0.732472 & 1.433740 & 4.099203 \\ 42 & 1 & 2.030210 & 2.040128 & 3.049761 \\ 43 & 1 & 0.324233 & 2.429338 & 2.683684\end{array}$

IRC path for $\mathrm{TS}_{2}-$ II:

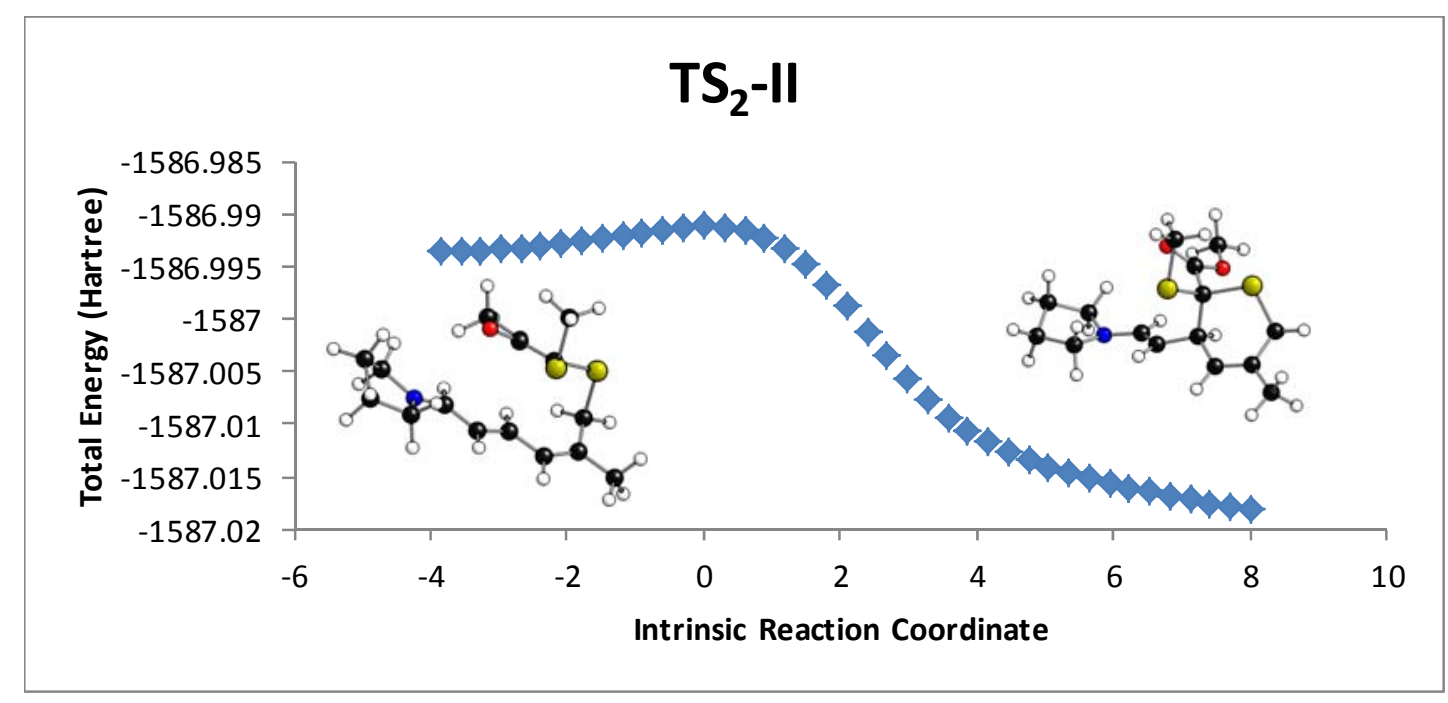


$C_{1}$

$\mathrm{HF}=-1587.0211442$ hartrees

Imaginary Frequencies: none found

Zero-point correction $=0.364160$ (Hartree/Particle)

Temperature: 298.150 Kelvin. Pressure: 1.00000 Atm.

Sum of electronic and thermal Free Energies $=-1586.709780$ hartrees

Coordinates:

\begin{tabular}{|c|c|c|c|c|}
\hline \multirow{2}{*}{$\begin{array}{l}\text { Center } \\
\text { Number }\end{array}$} & \multicolumn{2}{|c|}{ Atomic } & \multicolumn{2}{|c|}{ Coordinates (Angstroms) } \\
\hline & & & $x$ & Z \\
\hline 1 & 6 & 3.748576 & -1.695675 & -2.385104 \\
\hline 2 & 1 & 3.215335 & -2.576123 & -2.744509 \\
\hline 3 & 1 & 4.518588 & -2.022030 & -1.679742 \\
\hline 4 & 1 & 4.263299 & -1.234225 & -3.232497 \\
\hline 5 & 6 & 2.817494 & -0.724345 & -1.728096 \\
\hline 6 & 6 & 1.521595 & -0.941079 & -1.542927 \\
\hline 7 & 1 & 1.063450 & -1.863689 & -1.881137 \\
\hline 8 & 6 & 0.618796 & 0.054662 & -0.856799 \\
\hline 9 & 1 & 0.619371 & 0.967257 & -1.460576 \\
\hline 10 & 6 & -0.789514 & -0.452772 & -0.753787 \\
\hline 11 & 1 & -0.916775 & $5-1.475634$ & -0.416546 \\
\hline 12 & 6 & -1.857562 & 0.317401 & -1.016993 \\
\hline 13 & 1 & -1.706960 & 1.336191 & -1.362946 \\
\hline 14 & 6 & -3.563999 & -1.272571 & -0.252203 \\
\hline 15 & 6 & -4.258154 & 0.923715 & -0.993992 \\
\hline 16 & 6 & -5.084958 & -1.156431 & -0.154238 \\
\hline 17 & 1 & -3.245276 & -2.143881 & L -0.832119 \\
\hline 18 & 1 & -3.097224 & -1.347699 & 0.740128 \\
\hline 19 & 6 & -5.311682 & 0.353264 & -0.046432 \\
\hline 20 & 1 & -3.926740 & 1.922112 & -0.702607 \\
\hline 21 & 1 & -4.644325 & 0.978560 & -2.018540 \\
\hline 22 & 1 & -5.487261 & -1.711386 & 0.692017 \\
\hline 23 & 1 & -5.549656 & -1.542403 & $3-1.064345$ \\
\hline 24 & 1 & -5.112399 & 0.688813 & 0.973658 \\
\hline 25 & 1 & -6.323579 & 0.655185 & -0.314334 \\
\hline 26 & 7 & -3.162446 & -0.035583 & $3-0.897524$ \\
\hline 27 & 6 & 3.398758 & 0.549500 & -1.196111 \\
\hline 28 & 1 & 3.044781 & 1.416658 & -1.757553 \\
\hline 29 & 1 & 4.487958 & 0.550482 & -1.231315 \\
\hline 30 & 6 & 0.992264 & -2.836513 & 2.126135 \\
\hline
\end{tabular}




$\begin{array}{lcccc}31 & 1 & 1.399021 & -3.723788 & 1.650091 \\ 32 & 1 & -0.081026 & -2.946538 & 2.281742 \\ 33 & 1 & 1.481578 & -2.660942 & 3.083524 \\ 34 & 8 & 1.257418 & -1.761321 & 1.222394 \\ 35 & 6 & 0.857448 & -0.555570 & 1.608921 \\ 36 & 8 & 0.312881 & -0.340838 & 2.661327 \\ 37 & 6 & 1.160877 & 0.515588 & 0.555771 \\ 38 & 16 & 2.985954 & 0.806711 & 0.574650 \\ 39 & 16 & 0.294724 & 2.032290 & 1.163862 \\ 40 & 6 & 0.809179 & 3.234297 & -0.090547 \\ 41 & 1 & 0.518138 & 4.210605 & 0.294322 \\ 42 & 1 & 1.892273 & 3.210294 & -0.212649 \\ 43 & 1 & 0.316598 & 3.073709 & -1.048960\end{array}$


$\mathrm{C}_{11}$

$\mathrm{HF}=-1587.023792$ hartrees

Imaginary Frequencies: none found

Zero-point correction $=0.364913$ (Hartree/Particle)

Temperature: 298.150 Kelvin. Pressure: 1.00000 Atm.

Sum of electronic and thermal Free Energies $=-1586.710616$ hartrees

Coordinates:

\begin{tabular}{|c|c|c|c|c|}
\hline Center & & & oordinates (A & Angstroms \\
\hline Number & & & $x$ & Z \\
\hline 1 & 6 & 3.800428 & -2.529356 & -1.531648 \\
\hline 2 & 1 & 3.346145 & -3.513095 & -1.410256 \\
\hline 3 & 1 & 4.638796 & -2.448238 & -0.832906 \\
\hline 4 & 1 & 4.216094 & -2.460304 & -2.540508 \\
\hline 5 & 6 & 2.808050 & -1.437366 & -1.279466 \\
\hline 6 & 6 & 1.562318 & -1.639248 & -0.862313 \\
\hline 7 & 1 & 1.181787 & -2.642346 & -0.702079 \\
\hline 8 & 6 & 0.642286 & -0.482545 & -0.582759 \\
\hline 9 & 1 & 0.586601 & 0.116037 & -1.496118 \\
\hline 10 & 6 & -0.753491 & -0.886793 & -0.219204 \\
\hline 11 & 1 & -0.871240 & -1.589695 & 0.597191 \\
\hline 12 & 6 & -1.827311 & -0.369764 & -0.839961 \\
\hline 13 & 1 & -1.681446 & 0.343117 & -1.647373 \\
\hline 14 & 6 & -3.538069 & -1.452445 & 0.559510 \\
\hline 15 & 6 & -4.242150 & 0.078167 & -1.180984 \\
\hline 16 & 6 & -5.065064 & -1.420003 & 0.493724 \\
\hline 17 & 1 & -3.135645 & -2.466835 & 0.478471 \\
\hline 18 & 1 & -3.159603 & -1.021089 & 1.496390 \\
\hline 19 & 6 & -5.357574 & -0.060920 & -0.146473 \\
\hline 20 & 1 & -3.978752 & 1.120207 & -1.374988 \\
\hline 21 & 1 & -4.532373 & -0.381942 & -2.133159 \\
\hline 22 & 1 & -5.524636 & -1.540224 & 1.473677 \\
\hline 23 & 1 & -5.427652 & -2.223297 & -0.151613 \\
\hline 24 & 1 & -5.269886 & 0.730344 & 0.601395 \\
\hline 25 & 1 & -6.349528 & -0.000545 & -0.59261 \\
\hline 26 & 7 & -3.129554 & -0.637952 & -0.56980 \\
\hline 27 & 6 & 3.266187 & -0.017438 & -1.441593 \\
\hline 28 & 1 & 2.747538 & 0.488311 & -2.258360 \\
\hline 29 & 1 & 4.337313 & 0.048030 & -1.630196 \\
\hline 30 & 6 & -0.426532 & 3.480818 & -0.99884 \\
\hline
\end{tabular}




$\begin{array}{lcccc}31 & 1 & -0.452356 & 3.662354 & -2.069310 \\ 32 & 1 & 0.074442 & 4.301228 & -0.486054 \\ 33 & 1 & -1.437109 & 3.369988 & -0.606066 \\ 34 & 8 & 0.306448 & 2.264661 & -0.832699 \\ 35 & 6 & 0.448109 & 1.828312 & 0.417579 \\ 36 & 8 & 0.005134 & 2.408312 & 1.374250 \\ 37 & 6 & 1.243565 & 0.517980 & 0.479093 \\ 38 & 16 & 3.007138 & 0.932764 & 0.110341 \\ 39 & 16 & 1.101066 & -0.022778 & 2.237591 \\ 40 & 6 & 2.047841 & -1.568334 & 2.233051 \\ 41 & 1 & 2.201871 & -1.818681 & 3.282006 \\ 42 & 1 & 1.507838 & -2.375821 & 1.743150 \\ 43 & 1 & 3.013202 & -1.421783 & 1.750368\end{array}$




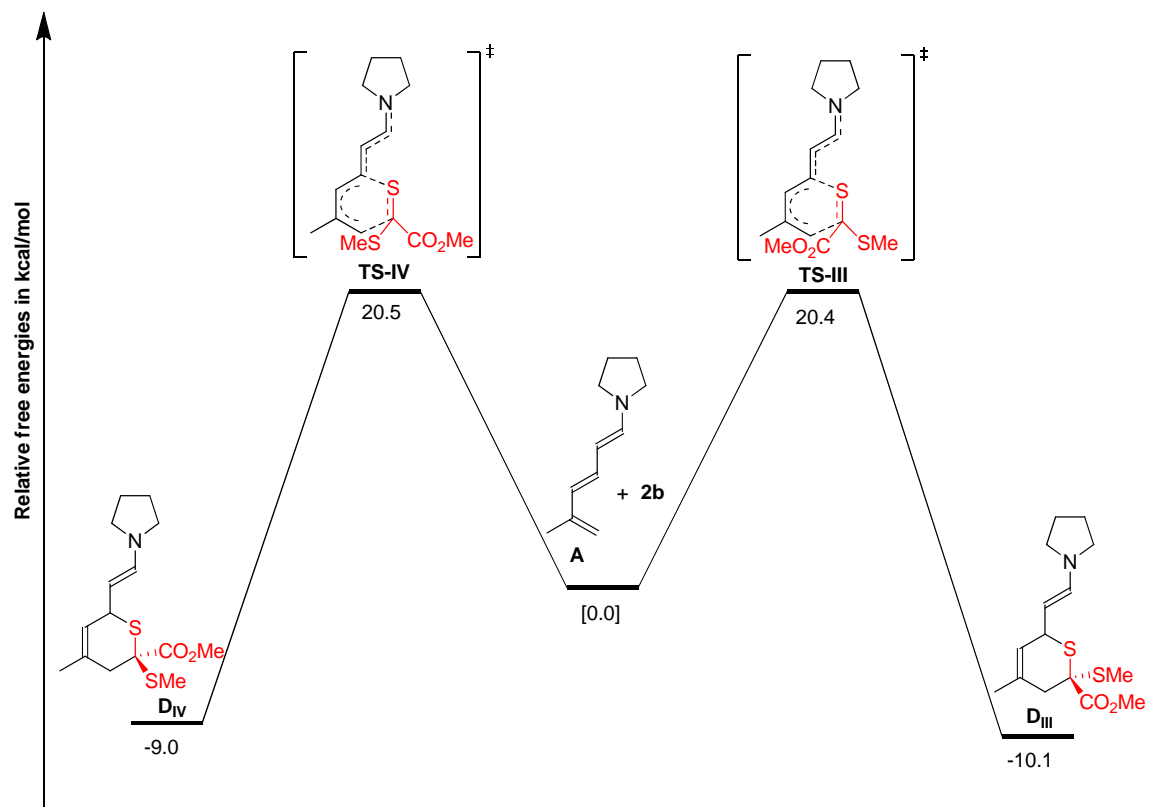

Figure S2: Energy profiles for pathways III-IV: the formal cycloaddition of $\mathbf{A}$ and $\mathbf{2 b}$ (initial attack at carbon). Reported values in plain text are wB97xd/tzvp free energies in solvent (IEFPCM, $\mathrm{CHCl}_{3}$ ), relative to $\mathbf{A}+\mathbf{2 b}$. 
TS-III

$\mathrm{HF}=-1586.9742041$ hartrees

Imaginary Frequencies: 1 (-444.9298 1/cm)

Zero-point correction $=0.360882$ (Hartree/Particle)

Temperature: 298.150 Kelvin. Pressure: 1.00000 Atm.

Sum of electronic and thermal Free Energies $=-1586.667338$ hartrees

Coordinates:

\begin{tabular}{ccccc} 
Center & \multicolumn{2}{c}{ Atomic } & \multicolumn{3}{c}{ Coordinates (Angstroms) } \\
Number & Number & X & $Y$ & $Z$ \\
---------------------------------------- \\
1 & 6 & 2.400952 & -2.638355 & -1.385093 \\
2 & 1 & 2.306039 & -3.194165 & -0.450187 \\
3 & 1 & 3.444788 & -2.354304 & -1.519083 \\
4 & 1 & 2.129263 & -3.316034 & -2.198329 \\
5 & 6 & 1.494433 & -1.437589 & -1.386524 \\
6 & 6 & 0.157911 & -1.636964 & -1.011136 \\
7 & 1 & -0.125199 & -2.640694 & -0.705672 \\
8 & 6 & -0.816407 & -0.660280 & -0.948506 \\
9 & 1 & -0.589095 & 0.330616 & -1.325730 \\
10 & 6 & -2.140278 & -0.882538 & -0.519783 \\
11 & 1 & -2.412286 & -1.863095 & -0.147414 \\
12 & 6 & -3.060300 & 0.133696 & -0.535321 \\
13 & 1 & -2.753529 & 1.115357 & -0.885600 \\
14 & 6 & -4.936270 & -1.165972 & 0.377397 \\
15 & 6 & -5.258876 & 1.183698 & -0.148073 \\
16 & 6 & -6.405338 & -0.787729 & 0.565866 \\
17 & 1 & -4.798126 & -1.993132 & -0.322290 \\
18 & 1 & -4.457702 & -1.437189 & 1.323880 \\
19 & 6 & -6.357268 & 0.723582 & 0.804028 \\
20 & 1 & -4.755958 & 2.093451 & 0.178774 \\
21 & 1 & -5.654752 & 1.344541 & -1.155753 \\
22 & 1 & -6.866813 & -1.334545 & 1.385980 \\
23 & 1 & -6.965513 & -1.005696 & -0.345568 \\
24 & 1 & -6.068402 & 0.934566 & 1.835578 \\
25 & 1 & -7.307358 & 1.217137 & 0.607136 \\
26 & 7 & -4.326853 & 0.053904 & -0.146205 \\
27 & 6 & 2.025803 & -0.179413 & -1.625988 \\
28 & 1 & 1.373933 & 0.634054 & -1.910356 \\
29 & 1 & 3.038422 & -0.100067 & -2.000441 \\
30 & 6 & 4.173058 & -2.094109 & 1.680451
\end{tabular}




$\begin{array}{lcccc}31 & 1 & 3.698557 & -2.913905 & 2.212196 \\ 32 & 1 & 4.907812 & -1.603258 & 2.317984 \\ 33 & 1 & 4.664542 & -2.462248 & 0.779764 \\ 34 & 8 & 3.116370 & -1.193058 & 1.345798 \\ 35 & 6 & 3.467693 & -0.112416 & 0.659392 \\ 36 & 8 & 4.609137 & 0.131722 & 0.343731 \\ 37 & 6 & 2.299590 & 0.778445 & 0.303179 \\ 38 & 16 & 0.844608 & 0.692911 & 1.151622 \\ 39 & 16 & 2.971402 & 2.349460 & -0.308510 \\ 40 & 6 & 1.467807 & 3.333912 & -0.498931 \\ 41 & 1 & 1.796369 & 4.293143 & -0.897939 \\ 42 & 1 & 0.777808 & 2.868952 & -1.202943 \\ 43 & 1 & 0.969895 & 3.474126 & 0.457678\end{array}$

IRC path for TS-III:

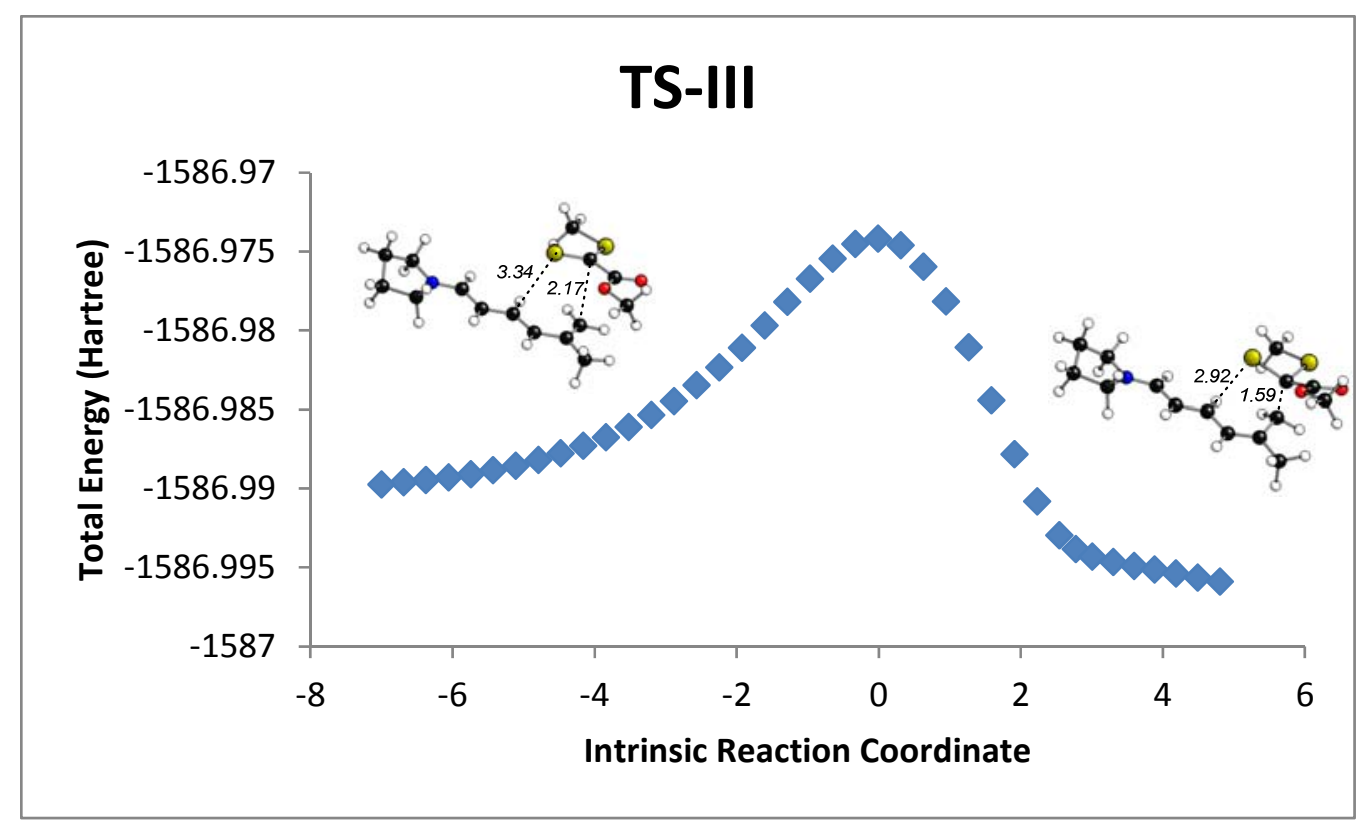


TS-IV

$\mathrm{HF}=-1586.9736831$ hartrees

Imaginary Frequencies: 1 (-423.9000 1/cm)

Zero-point correction $=0.360803$ (Hartree/Particle)

Temperature: 298.150 Kelvin. Pressure: 1.00000 Atm.

Sum of electronic and thermal Free Energies $=-1586.667226$ hartrees

Coordinates:

\begin{tabular}{|c|c|c|c|c|}
\hline Center & & & oordinates (A & Angstrom \\
\hline Number & & & $x$ & Z \\
\hline 1 & 6 & 2.302974 & -2.792868 & -1.625721 \\
\hline 2 & 1 & 2.515440 & -2.976523 & -2.682111 \\
\hline 3 & 1 & 1.805156 & -3.675800 & -1.224035 \\
\hline 4 & 1 & 3.261076 & -2.664419 & -1.117891 \\
\hline 5 & 6 & 1.462575 & -1.553782 & -1.475015 \\
\hline 6 & 6 & 0.102216 & -1.698422 & -1.162014 \\
\hline 7 & 1 & -0.270034 & -2.707689 & -1.010620 \\
\hline 8 & 6 & -0.792869 & -0.661481 & -0.983723 \\
\hline 9 & 1 & -0.470526 & 0.349200 & -1.208503 \\
\hline 10 & 6 & -2.144925 & -0.823351 & -0.620772 \\
\hline 11 & 1 & -2.516376 & $5-1.816988$ & -0.399393 \\
\hline 12 & 6 & -2.967295 & 0.268007 & -0.510491 \\
\hline 13 & 1 & -2.557297 & 1.254078 & -0.709686 \\
\hline 14 & 6 & -4.991096 & $5-0.951419$ & 0.173647 \\
\hline 15 & 6 & -5.068762 & 1.467061 & -0.038586 \\
\hline 16 & 6 & -6.426102 & -0.458583 & 0.359147 \\
\hline 17 & 1 & -4.897717 & -1.693181 & -0.622522 \\
\hline 18 & 1 & -4.584967 & -1.387115 & 1.092358 \\
\hline 19 & 6 & -6.250509 & 0.995951 & 0.802067 \\
\hline 20 & 1 & -4.498566 & 2.269467 & 0.429132 \\
\hline 21 & 1 & -5.398429 & 1.802524 & -1.026986 \\
\hline 22 & 1 & -6.976713 & -1.062025 & 1.078417 \\
\hline 23 & 1 & -6.957994 & -0.497605 & -0.593525 \\
\hline 24 & 1 & -5.995229 & 1.041335 & 1.862654 \\
\hline 25 & 1 & -7.140375 & 1.601123 & 0.637996 \\
\hline 26 & 7 & -4.248445 & 0.260211 & -0.163081 \\
\hline 27 & 6 & 2.090088 & -0.319745 & $-1.54909 €$ \\
\hline 28 & 1 & 1.511418 & 0.570619 & -1.750304 \\
\hline 29 & 1 & 3.122991 & -0.291192 & -1.876293 \\
\hline 30 & 6 & 2.825994 & 3.657569 & -0.80331 \\
\hline
\end{tabular}




$\begin{array}{lcccc}31 & 1 & 2.001662 & 4.335506 & -1.005003 \\ 32 & 1 & 3.373158 & 3.443627 & -1.721738 \\ 33 & 1 & 3.505248 & 4.094060 & -0.071807 \\ 34 & 8 & 2.226974 & 2.467246 & -0.286866 \\ 35 & 6 & 3.067526 & 1.497954 & 0.062282 \\ 36 & 8 & 4.268354 & 1.600268 & -0.023838 \\ 37 & 6 & 2.377501 & 0.250075 & 0.565427 \\ 38 & 16 & 0.854188 & 0.329658 & 1.281934 \\ 39 & 16 & 3.653385 & -0.893718 & 1.146866 \\ 40 & 6 & 2.649160 & -2.198063 & 1.897010 \\ 41 & 1 & 2.114645 & -1.822749 & 2.766461 \\ 42 & 1 & 1.936475 & -2.601746 & 1.180581 \\ 43 & 1 & 3.353428 & -2.974565 & 2.194124\end{array}$

IRC path for TS-IV:

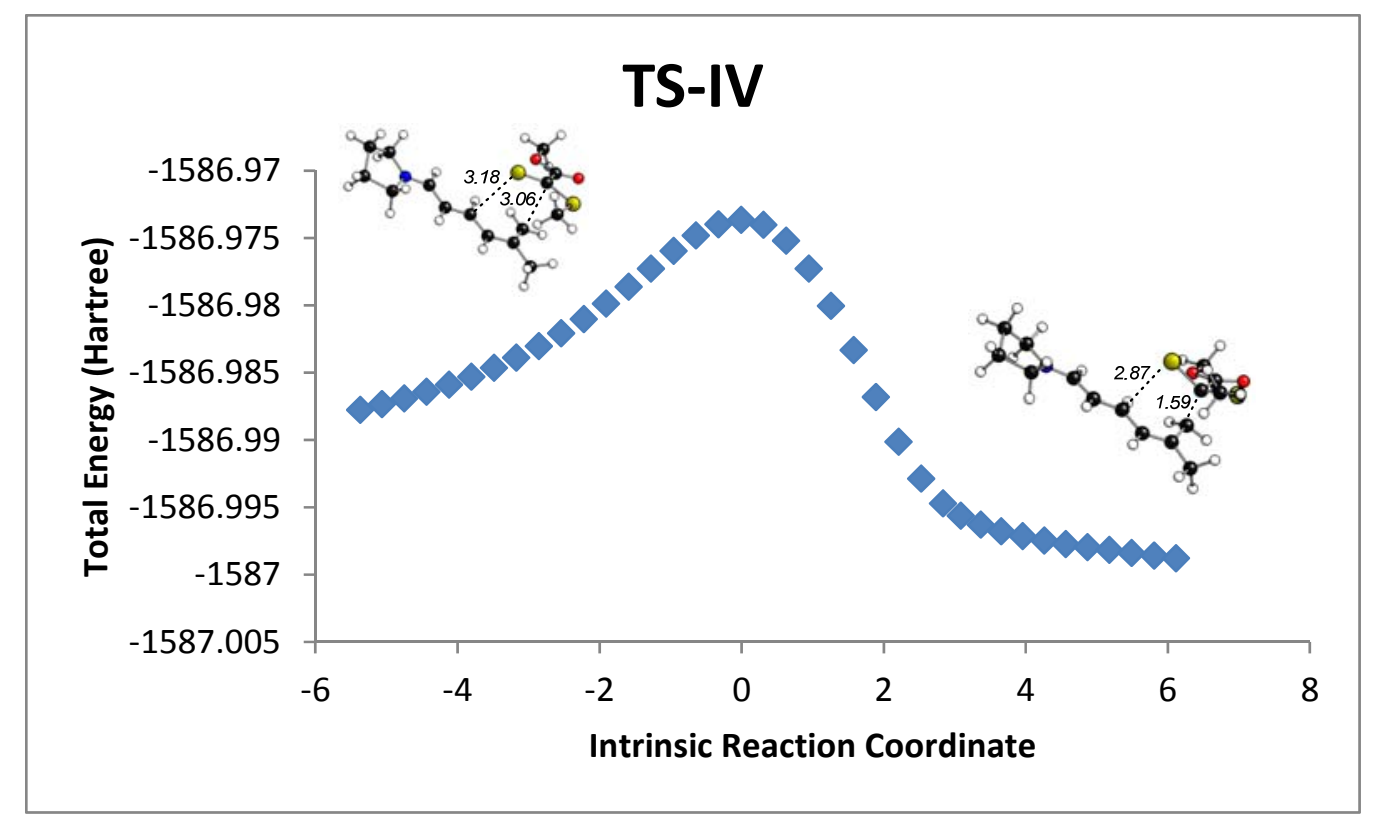


D III

$H F=-1587.0255809$ hartrees

Imaginary Frequencies: none found

Zero-point correction $=0.363855$ (Hartree/Particle)

Temperature: 298.150 Kelvin. Pressure: 1.00000 Atm.

Sum of electronic and thermal Free Energies $=-1586.715990$ hartrees

Coordinates:

\begin{tabular}{|c|c|c|c|c|}
\hline \multirow{2}{*}{$\begin{array}{l}\text { Center } \\
\text { Number }\end{array}$} & \multicolumn{2}{|c|}{ Atomic } & \multicolumn{2}{|c|}{ Coordinates (Angstroms) } \\
\hline & & & $x$ & Z \\
\hline 1 & 6 & -2.414354 & -2.405167 & 2.208884 \\
\hline 2 & 1 & -1.790376 & -3.282162 & 2.383306 \\
\hline 3 & 1 & -3.236923 & -2.683484 & 1.546332 \\
\hline 4 & 1 & -2.855947 & -2.102869 & 3.163021 \\
\hline 5 & 6 & -1.626463 & -1.280871 & 1.613521 \\
\hline 6 & 6 & -0.326686 & -1.358903 & 1.350007 \\
\hline 7 & 1 & 0.221430 & -2.277634 & 1.532999 \\
\hline 8 & 6 & 0.452360 & -0.203414 & 0.801854 \\
\hline 9 & 1 & 0.513412 & 0.591583 & 1.550135 \\
\hline 10 & & 1.818745 & -0.550331 & 0.322512 \\
\hline 11 & $\perp$ & 1.915341 & -1.450431 & -0.274177 \\
\hline 12 & 6 & 2.883111 & 0.237388 & 0.568854 \\
\hline 13 & 1 & 2.748837 & 1.142137 & 1.156354 \\
\hline 14 & 6 & 4.537431 & -1.081938 & -0.690958 \\
\hline 15 & 6 & 5.252687 & 0.955246 & 0.409578 \\
\hline 16 & 6 & 6.052355 & -0.924650 & -0.832682 \\
\hline 17 & 1 & 4.257490 & -2.036044 & -0.233539 \\
\hline 18 & 1 & 4.025900 & -1.017352 & -1.660115 \\
\hline 19 & 6 & 6.269775 & 0.581500 & -0.665966 \\
\hline 20 & 1 & 4.917232 & 1.991107 & 0.332227 \\
\hline 21 & 1 & 5.672217 & 0.804980 & 1.411850 \\
\hline 22 & 1 & 6.421808 & -1.309197 & -1.782162 \\
\hline 23 & 1 & 6.558657 & -1.464756 & -0.029527 \\
\hline 24 & 1 & 6.032106 & 1.100649 & -1.597008 \\
\hline 25 & 1 & 7.290200 & 0.836808 & -0.383123 \\
\hline 26 & 7 & 4.153418 & 0.033228 & 0.154807 \\
\hline 27 & 6 & -2.359541 & 0.010404 & 1.344551 \\
\hline 28 & 1 & -1.990110 & 0.765005 & 2.043233 \\
\hline 29 & 1 & -3.425188 & $3-0.106660$ & 1.544405 \\
\hline 30 & 6 & -3.925551 & -2.278330 & -1.691972 \\
\hline
\end{tabular}




$\begin{array}{lcccc}31 & 1 & -4.073899 & -3.229054 & -1.188376 \\ 32 & 1 & -3.294639 & -2.407728 & -2.570624 \\ 33 & 1 & -4.881990 & -1.850335 & -1.989918 \\ 34 & 8 & -3.277083 & -1.434615 & -0.735306 \\ 35 & 6 & -2.980120 & -0.204214 & -1.138082 \\ 36 & 8 & -3.268320 & 0.229451 & -2.224062 \\ 37 & 6 & -2.210103 & 0.585373 & -0.077299 \\ 38 & 16 & -0.450457 & 0.567571 & -0.650218 \\ 39 & 16 & -2.921369 & 2.291229 & -0.180466 \\ 40 & 6 & -1.883278 & 3.173173 & 1.014335 \\ 41 & 1 & -2.053775 & 4.233767 & 0.836561 \\ 42 & 1 & -2.141613 & 2.940891 & 2.045904 \\ 43 & 1 & -0.833872 & 2.947096 & 0.821639\end{array}$


$D_{\text {IV }}$

$\mathrm{HF}=-1587.024408$ hartrees

Imaginary Frequencies: none found

Zero-point correction $=0.364332$ (Hartree/Particle)

Temperature: 298.150 Kelvin. Pressure: 1.00000 Atm.

Sum of electronic and thermal Free Energies $=-1586.714105$ hartrees

Coordinates:

\begin{tabular}{cccccc} 
Center & \multicolumn{2}{c}{ Atomic } & \multicolumn{3}{c}{ Coordinates (Angstroms) } \\
Number & Number & X & $Y$ & $Z$ \\
---------------------------------- \\
1 & 6 & -2.287643 & -2.922762 & 1.988729 \\
2 & 1 & -2.706224 & -2.684675 & 2.970955 \\
3 & 1 & -1.595023 & -3.756563 & 2.105480 \\
4 & 1 & -3.124480 & -3.248362 & 1.364710 \\
5 & 6 & -1.606044 & -1.722566 & 1.406952 \\
6 & 6 & -0.298979 & -1.670330 & 1.169954 \\
7 & 1 & 0.330477 & -2.537306 & 1.344493 \\
8 & 6 & 0.376261 & -0.431670 & 0.663065 \\
9 & 1 & 0.317647 & 0.364779 & 1.409552 \\
10 & 6 & 1.790399 & -0.633708 & 0.242050 \\
11 & 1 & 2.003442 & -1.509488 & -0.361026 \\
12 & 6 & 2.760274 & 0.246534 & 0.555259 \\
13 & 1 & 2.510357 & 1.119266 & 1.153399 \\
14 & 6 & 4.593352 & -0.869213 & -0.653534 \\
15 & 6 & 5.057428 & 1.181910 & 0.549848 \\
16 & 6 & 6.091306 & -0.563659 & -0.714081 \\
17 & 1 & 4.386839 & -1.859947 & -0.236381 \\
18 & 1 & 4.123509 & -0.823298 & -1.644470 \\
19 & 6 & 6.156478 & 0.948804 & -0.483835 \\
20 & 1 & 4.630248 & 2.184876 & 0.495744 \\
21 & 1 & 5.439456 & 1.026357 & 1.566238 \\
22 & 1 & 6.538973 & -0.873063 & -1.657266 \\
23 & 1 & 6.609889 & -1.085705 & 0.093125 \\
24 & 1 & 5.915980 & 1.480378 & -1.407100 \\
25 & 1 & 7.132337 & 1.287328 & -0.137980 \\
26 & 7 & 4.062577 & 0.176690 & 0.201019 \\
27 & 6 & -2.445151 & -0.496769 & 1.160214 \\
28 & 1 & -2.168065 & 0.259073 & 1.898148 \\
29 & 1 & -3.502891 & -0.717739 & 1.311319 \\
30 & 6 & -2.558012 & 3.657094 & 0.967879
\end{tabular}




$\begin{array}{lcccc}31 & 1 & -2.047959 & 4.002485 & 1.862206 \\ 32 & 1 & -3.632374 & 3.812707 & 1.059182 \\ 33 & 1 & -2.186359 & 4.184526 & 0.089999 \\ 34 & 8 & -2.259733 & 2.261303 & 0.870153 \\ 35 & 6 & -2.771641 & 1.620654 & -0.178868 \\ 36 & 8 & -3.494476 & 2.143757 & -0.986773 \\ 37 & 6 & -2.306866 & 0.160228 & -0.238166 \\ 38 & 16 & -0.556644 & 0.211477 & -0.827441 \\ 39 & 16 & -3.378667 & -0.619334 & -1.525694 \\ 40 & 6 & -2.679592 & -2.289685 & -1.619896 \\ 41 & 1 & -1.595831 & -2.232315 & -1.712071 \\ 42 & 1 & -2.951354 & -2.900818 & -0.763162 \\ 43 & 1 & -3.097398 & -2.733257 & -2.522533\end{array}$


The s-cis-conformer of the dithioester (s-cis-2b) is energetically close to $\mathbf{2} \mathbf{b}$. The approach of $\mathbf{s}-c i s-\mathbf{2} \mathbf{b}$ to $\mathbf{A}$ was also investigated. The energy profiles for these alternative pathways are described below. The activation energies are higher than those obtained for $\mathbf{2} \mathbf{b}$.

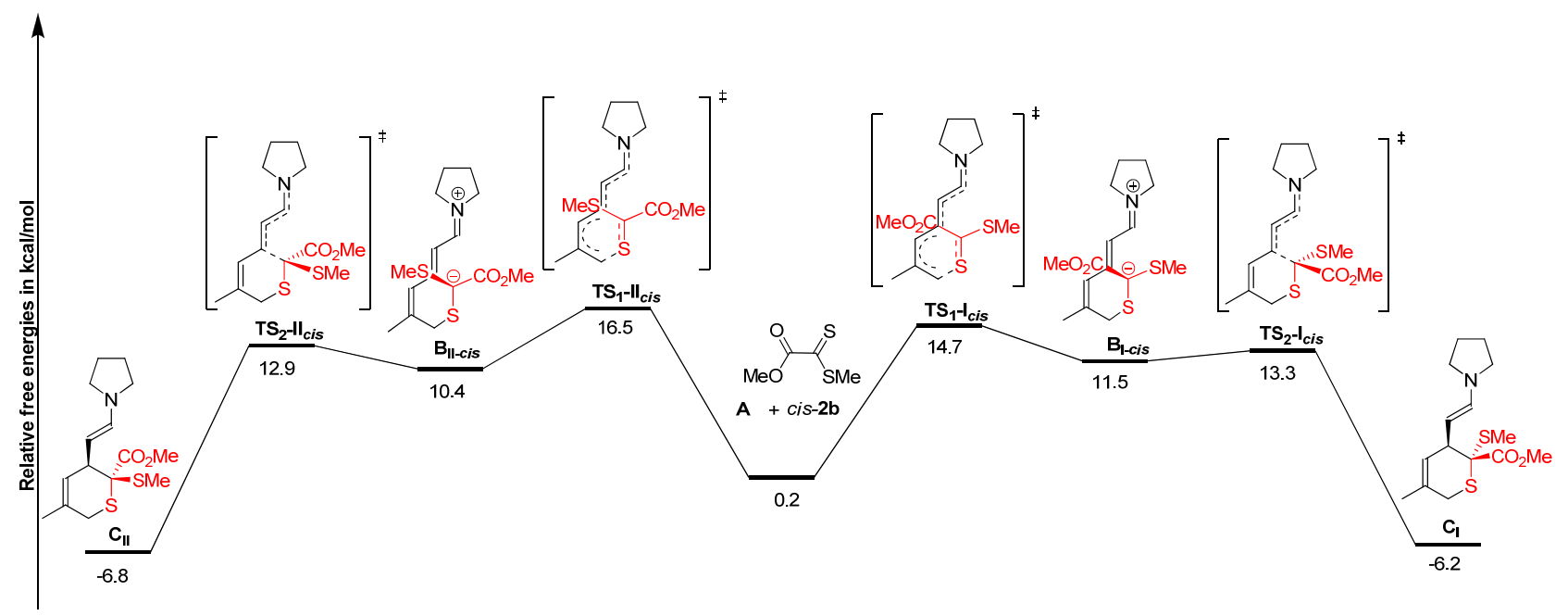

Figure S3: Energy profiles for reaction pathways $\mathrm{I}_{\mathrm{c}}-\mathrm{Il}_{\mathrm{c}}$ : the formal cycloaddition of $\mathbf{A}$ and $\mathrm{s}-\mathrm{cis}-\mathbf{2 b}$ (initial attack at sulfur). Reported values in plain text are wB97xd/tzvp free energies in solvent (IEFPCM, $\mathrm{CHCl}_{3}$ ), relative to $\mathbf{A}+\mathbf{2} \mathbf{b}$. 
s-cis-2b

$\mathrm{HF}=-1102.8696264$ hartrees

Imaginary Frequencies: none found

Zero-point correction $=0.099593$ (Hartree/Particle)

Temperature: 298.150 Kelvin. Pressure: 1.00000 Atm.

Sum of electronic and thermal Free Energies $=-1102.806131$ hartrees

Coordinates:

\begin{tabular}{cccccc} 
Center & \multicolumn{2}{c}{ Atomic } & \multicolumn{4}{c}{ Coordinates (Angstroms) } \\
Number & Number & X & $Y$ & $Z$ \\
\hline$-y$ & 6 & -3.144247 & -0.779049 & -0.278827 \\
2 & 1 & -3.421900 & -1.680418 & -0.815749 \\
3 & 1 & -3.614848 & 0.095013 & -0.726540 \\
4 & 1 & -3.428482 & -0.854173 & 0.769895 \\
5 & 8 & -1.716639 & -0.686677 & -0.397431 \\
6 & 6 & -1.144683 & 0.363847 & 0.176048 \\
7 & 8 & -1.726438 & 1.232104 & 0.763639 \\
8 & 6 & 0.367628 & 0.332114 & -0.000209 \\
9 & 16 & 1.182722 & 1.717197 & -0.295128 \\
10 & 16 & 1.023119 & -1.262615 & 0.182720 \\
11 & 6 & 2.800656 & -0.979448 & 0.037513 \\
12 & 1 & 3.136076 & -0.283782 & 0.804309 \\
13 & 1 & 3.266612 & -1.952838 & 0.178281 \\
14 & 1 & 3.037589 & -0.585295 & -0.948487
\end{tabular}


$\mathrm{TS}_{1}-\mathrm{I}_{\text {cis }}$

$\mathrm{HF}=-1586.9833399$ hartrees

Imaginary Frequencies: 1 (-273.7760 1/cm)

Zero-point correction $=0.360687$ (Hartree/Particle)

Temperature: 298.150 Kelvin. Pressure: 1.00000 Atm.

Sum of electronic and thermal Free Energies $=-1586.676441$ hartrees

Coordinates:

\begin{tabular}{|c|c|c|c|c|}
\hline \multirow{2}{*}{$\begin{array}{l}\text { Center } \\
\text { Number }\end{array}$} & \multicolumn{2}{|c|}{ Atomic } & \multicolumn{2}{|c|}{ Coordinates (Angstroms) } \\
\hline & & & $x$ & Z \\
\hline 1 & 6 & -3.638886 & 3.042524 & 0.072697 \\
\hline 2 & 1 & -3.521191 & 2.941565 & 1.154893 \\
\hline 3 & 1 & -4.692833 & 2.910463 & -0.168973 \\
\hline 4 & 1 & -3.343751 & 4.059547 & -0.193279 \\
\hline 5 & 6 & -2.771782 & 2.034725 & -0.630442 \\
\hline 6 & 6 & -1.378304 & 2.203551 & -0.528490 \\
\hline 7 & 1 & -1.031721 & 3.085206 & 0.003841 \\
\hline 8 & 6 & -0.430420 & 1.293571 & -0.929956 \\
\hline 9 & 1 & -0.744909 & 0.405135 & -1.467244 \\
\hline 10 & & 0.953178 & 1.420226 & -0.685964 \\
\hline 11 & $\perp$ & 1.316962 & 2.294517 & -0.158323 \\
\hline 12 & 6 & 1.805886 & 0.398092 & -1.008782 \\
\hline 13 & 1 & 1.406136 & -0.477834 & -1.510800 \\
\hline 14 & 6 & 3.827056 & 1.387492 & -0.016190 \\
\hline 15 & 6 & 3.957559 & -0.805370 & -1.050270 \\
\hline 16 & 6 & 5.276256 & 0.899552 & -0.016555 \\
\hline 17 & 1 & 3.704658 & 2.350765 & -0.516532 \\
\hline 18 & 1 & 3.427754 & 1.483865 & 0.999005 \\
\hline 19 & 6 & 5.143032 & -0.622694 & -0.108361 \\
\hline 20 & 1 & 3.416499 & -1.738036 & -0.889781 \\
\hline 21 & 1 & 4.276486 & -0.762179 & -2.096672 \\
\hline 22 & 1 & 5.818558 & 1.228907 & 0.867856 \\
\hline 23 & 1 & 5.797431 & 1.282681 & -0.895917 \\
\hline 24 & 1 & 4.908880 & -1.043220 & 0.871933 \\
\hline 25 & $\perp$ & 6.044133 & -1.107455 & -0.480027 \\
\hline 26 & 7 & 3.105080 & 0.339829 & -0.730710 \\
\hline 27 & 6 & -3.364635 & 0.916295 & -1.208104 \\
\hline 28 & 1 & -2.811467 & 0.315860 & -1.918887 \\
\hline 29 & 1 & -4.436615 & 0.924285 & -1.363856 \\
\hline 30 & 6 & 1.454255 & -0.588176 & 2.28836 \\
\hline
\end{tabular}




$\begin{array}{lcccc}31 & 1 & 2.377975 & -1.069566 & 1.976152 \\ 32 & 1 & 1.221985 & -0.852891 & 3.320246 \\ 33 & 1 & 1.548952 & 0.495092 & 2.207931 \\ 34 & 8 & 0.450972 & -1.067260 & 1.401351 \\ 35 & 6 & -0.781064 & -0.533143 & 1.544442 \\ 36 & 8 & -1.039389 & 0.265169 & 2.418204 \\ 37 & 6 & -1.710176 & -1.068903 & 0.538135 \\ 38 & 16 & -3.284989 & -0.563265 & 0.481962 \\ 39 & 16 & -1.005709 & -2.278687 & -0.585890 \\ 40 & 6 & -2.475280 & -2.958252 & -1.396717 \\ 41 & 1 & -2.104927 & -3.733502 & -2.066743 \\ 42 & 1 & -3.149966 & -3.397041 & -0.663414 \\ 43 & 1 & -3.006133 & -2.203951 & -1.972902\end{array}$


$\mathrm{TS}_{1}-\mathrm{II}_{\text {cis }}$

$\mathrm{HF}=-1586.981866$ hartrees

Imaginary Frequencies: 1 (-267.0372 1/cm)

Zero-point correction $=0.361221$ (Hartree $/$ Particle)

Temperature: 298.150 Kelvin. Pressure: 1.00000 Atm.

Sum of electronic and thermal Free Energies $=-1586.673600$ hartrees

Coordinates:

\begin{tabular}{cccccc} 
Center & \multicolumn{2}{c}{ Atomic } & \multicolumn{3}{c}{ Coordinates (Angstroms) } \\
Number & Number & X & $Y$ & $Z$ \\
--------------------------------- \\
1 & 6 & -3.896269 & 2.430861 & 0.641907 \\
2 & 1 & -4.782270 & 1.835215 & 0.414221 \\
3 & 1 & -4.148292 & 3.476972 & 0.446760 \\
4 & 1 & -3.674111 & 2.335601 & 1.705459 \\
5 & 6 & -2.733666 & 2.009865 & -0.212847 \\
6 & 6 & -1.448654 & 2.007229 & 0.344618 \\
7 & 1 & -1.347931 & 2.307835 & 1.383037 \\
8 & 6 & -0.320838 & 1.559035 & -0.309731 \\
9 & 1 & -0.405459 & 1.284440 & -1.355923 \\
10 & 6 & 0.964881 & 1.456515 & 0.248147 \\
11 & 1 & 1.111638 & 1.673735 & 1.299396 \\
12 & 6 & 2.013037 & 1.046180 & -0.537172 \\
13 & 1 & 1.830517 & 0.853967 & -1.590358 \\
14 & 6 & 3.691357 & 0.914306 & 1.254619 \\
15 & 6 & 4.341601 & 0.400575 & -1.026952 \\
16 & 6 & 5.190291 & 0.615846 & 1.194201 \\
17 & 1 & 3.474828 & 1.901127 & 1.669257 \\
18 & 1 & 3.144827 & 0.171663 & 1.845260 \\
19 & 6 & 5.337774 & -0.239181 & -0.066928 \\
20 & 1 & 3.962838 & -0.285518 & -1.785252 \\
21 & 1 & 4.781943 & 1.265725 & -1.532193 \\
22 & 1 & 5.543908 & 0.116028 & 2.093977 \\
23 & 1 & 5.750086 & 1.546308 & 1.081713 \\
24 & 1 & 5.046209 & -1.271696 & 0.137639 \\
25 & 1 & 6.350866 & -0.242519 & -0.465239 \\
26 & 7 & 3.261044 & 0.830483 & -0.138549 \\
27 & 6 & -2.991652 & 1.503330 & -1.488172 \\
28 & 1 & -2.218065 & 1.515592 & -2.242620 \\
29 & 1 & -3.997744 & 1.606338 & -1.879366 \\
30 & 6 & 1.695281 & -2.441052 & -0.328018 \\
& & & &
\end{tabular}




$\begin{array}{lcccc}31 & 1 & 2.195950 & -2.853007 & 0.544342 \\ 32 & 1 & 2.279143 & -1.618395 & -0.737992 \\ 33 & 1 & 1.575987 & -3.211070 & -1.090824 \\ 34 & 8 & 0.428518 & -1.987975 & 0.133343 \\ 35 & 6 & -0.398144 & -1.446841 & -0.785169 \\ 36 & 8 & -0.049049 & -1.274065 & -1.936496 \\ 37 & 6 & -1.706874 & -1.128775 & -0.209180 \\ 38 & 16 & -2.987329 & -0.711487 & -1.182999 \\ 39 & 16 & -1.840692 & -1.403302 & 1.562285 \\ 40 & 6 & -3.607980 & -1.170701 & 1.867894 \\ 41 & 1 & -3.742559 & -1.356461 & 2.933297 \\ 42 & 1 & -3.924748 & -0.156508 & 1.634188 \\ 43 & 1 & -4.203145 & -1.878962 & 1.294403\end{array}$


B I-cis $_{\text {s }}$

$H F=-1586.9925359$ hartrees

Imaginary Frequencies: none found

Zero-point correction $=0.363151$ (Hartree/Particle)

Temperature: 298.150 Kelvin. Pressure: 1.00000 Atm.

Sum of electronic and thermal Free Energies $=-1586.681483$ hartrees

Coordinates:

\begin{tabular}{ccccc} 
Center & \multicolumn{2}{c}{ Atomic } & \multicolumn{3}{c}{ Coordinates (Angstroms) } \\
Number & Number & X & $Y$ & $Z$ \\
-------------------------------- \\
1 & 6 & 3.834260 & -2.954418 & 0.259916 \\
2 & 1 & 4.394930 & -2.422490 & 1.033127 \\
3 & 1 & 4.561707 & -3.322228 & -0.468928 \\
4 & 1 & 3.333760 & -3.806341 & 0.717916 \\
5 & 6 & 2.868106 & -2.020332 & -0.400153 \\
6 & 6 & 1.534367 & -2.280622 & -0.378702 \\
7 & 1 & 1.194128 & -3.161032 & 0.157784 \\
8 & 6 & 0.533079 & -1.417687 & -0.885889 \\
9 & 1 & 0.820309 & -0.632764 & -1.572975 \\
10 & 6 & -0.806354 & -1.561263 & -0.587389 \\
11 & 1 & -1.127403 & -2.363798 & 0.065206 \\
12 & 6 & -1.704726 & -0.573162 & -0.977462 \\
13 & 1 & -1.332499 & 0.273782 & -1.545460 \\
14 & 6 & -3.669819 & -1.554669 & 0.122780 \\
15 & 6 & -3.861944 & 0.590278 & -1.011729 \\
16 & 6 & -5.126814 & -1.095455 & 0.115891 \\
17 & 1 & -3.526585 & -2.539009 & -0.323995 \\
18 & 1 & -3.251546 & -1.569809 & 1.132689 \\
19 & 6 & -5.022237 & 0.423462 & -0.037835 \\
20 & 1 & -3.326266 & 1.532056 & -0.902848 \\
21 & 1 & -4.196199 & 0.488191 & -2.047147 \\
22 & 1 & -5.650886 & -1.398249 & 1.020039 \\
23 & 1 & -5.649107 & -1.524920 & -0.740858 \\
24 & 1 & -4.777420 & 0.886013 & 0.920268 \\
25 & 1 & -5.937431 & 0.878182 & -0.411694 \\
26 & 7 & -2.976969 & -0.532383 & -0.669598 \\
27 & 6 & 3.433407 & -0.772836 & -0.937737 \\
28 & 1 & 2.940914 & -0.386665 & -1.828037 \\
29 & 1 & 4.502010 & -0.857656 & -1.127483 \\
30 & 6 & -1.505142 & 0.909007 & 2.129086
\end{tabular}




$\begin{array}{lcccc}31 & 1 & -2.449733 & 1.326006 & 1.782164 \\ 32 & 1 & -1.230649 & 1.367799 & 3.080534 \\ 33 & 1 & -1.605931 & -0.167363 & 2.279138 \\ 34 & 8 & -0.559457 & 1.203858 & 1.117551 \\ 35 & 6 & 0.703103 & 0.696272 & 1.314947 \\ 36 & 8 & 0.942652 & 0.045694 & 2.325462 \\ 37 & 6 & 1.594796 & 1.009729 & 0.247265 \\ 38 & 16 & 3.269195 & 0.567277 & 0.378192 \\ 39 & 16 & 1.091726 & 1.969619 & -1.150527 \\ 40 & 6 & 1.474427 & 3.681022 & -0.646736 \\ 41 & 1 & 1.229718 & 4.336832 & -1.482822 \\ 42 & 1 & 0.876794 & 3.957010 & 0.220670 \\ 43 & 1 & 2.534827 & 3.770422 & -0.414355\end{array}$


$\mathbf{B}_{\text {II-cis }}$

$\mathrm{HF}=-1586.9918164$ hartrees

Imaginary Frequencies: none found

Zero-point correction $=0.362740$ (Hartree/Particle)

Temperature: 298.150 Kelvin. Pressure: 1.00000 Atm.

Sum of electronic and thermal Free Energies $=-1586.683307$ hartrees

Coordinates:

\begin{tabular}{|c|c|c|c|c|}
\hline \multirow{2}{*}{$\begin{array}{l}\text { Center } \\
\text { Number }\end{array}$} & \multicolumn{2}{|c|}{ Atomic } & \multicolumn{2}{|c|}{ Coordinates (Angstroms) } \\
\hline & & & $x$ & Z \\
\hline 1 & 6 & 4.030081 & -2.667923 & 0.471819 \\
\hline 2 & 1 & 4.705289 & -1.918158 & 0.892085 \\
\hline 3 & 1 & 4.614053 & -3.269020 & -0.230575 \\
\hline 4 & 1 & 3.680636 & -3.313020 & 1.276999 \\
\hline 5 & 6 & 2.898439 & -1.992443 & -0.237196 \\
\hline 6 & 6 & 1.610213 & -2.210729 & 0.137668 \\
\hline 7 & 1 & 1.415258 & -2.866135 & 0.980877 \\
\hline 8 & 6 & 0.496513 & -1.568492 & -0.457637 \\
\hline 9 & 1 & 0.641014 & -1.057199 & -1.402065 \\
\hline 10 & 6 & -0.789755 & -1.634772 & 0.035910 \\
\hline 11 & 1 & -0.983680 & -2.123255 & 0.982490 \\
\hline 12 & 6 & -1.813315 & -0.991998 & -0.651164 \\
\hline 13 & 1 & -1.585590 & -0.532340 & -1.608148 \\
\hline 14 & 6 & -3.553770 & -1.351818 & 1.042672 \\
\hline 15 & 6 & -4.103305 & $5-0.189160$ & -1.019856 \\
\hline 16 & 6 & -5.042257 & $7-1.003203$ & 1.015123 \\
\hline 17 & 1 & -3.368638 & $3-2.420413$ & 1.157377 \\
\hline 18 & 1 & -3.020231 & L -0.820454 & 1.834912 \\
\hline 19 & 6 & -5.118236 & 0.183876 & 0.051578 \\
\hline 20 & 1 & -3.680920 & 0.664376 & -1.546025 \\
\hline 21 & 1 & -4.534190 & -0.878404 & -1.750865 \\
\hline 22 & 1 & -5.422176 & $5-0.772823$ & 2.008269 \\
\hline 23 & 1 & -5.615466 & $5-1.843500$ & 0.619403 \\
\hline 24 & 1 & -4.808449 & 1.102892 & 0.552837 \\
\hline 25 & 1 & -6.113356 & 0.334767 & -0.362126 \\
\hline 26 & 7 & -3.056911 & -0.880830 & -0.255836 \\
\hline 27 & 6 & 3.250355 & -0.978037 & -1.238307 \\
\hline 28 & 1 & 2.562921 & -0.888873 & -2.075226 \\
\hline 29 & 1 & 4.266093 & -1.095465 & -1.612110 \\
\hline 30 & 6 & -1.388168 & 2.455634 & -1.31890 \\
\hline
\end{tabular}




$\begin{array}{lcccc}31 & 1 & -2.302386 & 2.669260 & -0.767572 \\ 32 & 1 & -1.600881 & 1.787651 & -2.155711 \\ 33 & 1 & -0.977790 & 3.383167 & -1.722915 \\ 34 & 8 & -0.499036 & 1.867116 & -0.385239 \\ 35 & 6 & 0.697823 & 1.432593 & -0.893080 \\ 36 & 8 & 0.873734 & 1.424825 & -2.110057 \\ 37 & 6 & 1.612795 & 0.977580 & 0.099026 \\ 38 & 16 & 3.263504 & 0.704575 & -0.373089 \\ 39 & 16 & 1.180989 & 0.920872 & 1.812186 \\ 40 & 6 & 1.397717 & 2.654903 & 2.342107 \\ 41 & 1 & 1.147390 & 2.711716 & 3.402178 \\ 42 & 1 & 2.430568 & 2.970690 & 2.198829 \\ 43 & 1 & 0.728656 & 3.301595 & 1.776154\end{array}$


$\mathrm{TS}_{2}-\mathrm{I}_{\text {cis }}$

$\mathrm{HF}=-1586.990177$ hartrees

Imaginary Frequencies: 1 (-199.4218 1/cm)

Zero-point correction $=0.362639$ (Hartree/Particle)

Temperature: 298.150 Kelvin. Pressure: 1.00000 Atm.

Sum of electronic and thermal Free Energies $=-1586.678628$ hartrees

Coordinates:

\begin{tabular}{|c|c|c|c|c|}
\hline \multirow{2}{*}{$\begin{array}{l}\text { Center } \\
\text { Number }\end{array}$} & \multicolumn{2}{|c|}{ Atomic } & \multicolumn{2}{|c|}{ Coordinates (Angstroms) } \\
\hline & & & $x$ & Z \\
\hline 1 & 6 & 3.928474 & -2.924898 & 0.248809 \\
\hline 2 & 1 & 4.528166 & -2.413135 & 1.006961 \\
\hline 3 & 1 & 4.621834 & -3.312579 & -0.502292 \\
\hline 4 & 1 & 3.419294 & -3.764548 & 0.720850 \\
\hline 5 & 6 & 2.961507 & -1.965025 & -0.372623 \\
\hline 6 & 6 & 1.625809 & -2.134603 & -0.266175 \\
\hline 7 & 1 & 1.256104 & -2.969666 & 0.321211 \\
\hline 8 & 6 & 0.643941 & -1.222425 & -0.770743 \\
\hline 9 & 1 & 0.869391 & -0.682625 & -1.681691 \\
\hline 10 & 6 & -0.714286 & $5-1.389557$ & -0.428530 \\
\hline 11 & 1 & -0.961002 & -2.076276 & 0.372675 \\
\hline 12 & 6 & -1.691950 & -0.608054 & -0.989453 \\
\hline 13 & 1 & -1.412569 & 0.124084 & -1.740265 \\
\hline 14 & 6 & -3.558946 & $5-1.502639$ & 0.336227 \\
\hline 15 & 6 & -3.969996 & 0.289022 & -1.250435 \\
\hline 16 & 6 & -5.054264 & -1.188676 & 0.281587 \\
\hline 17 & 1 & -3.335814 & -2.548339 & 0.114217 \\
\hline 18 & 1 & -3.127609 & -1.266637 & 1.313858 \\
\hline 19 & 6 & -5.099739 & 0.254441 & -0.227175 \\
\hline 20 & 1 & -3.534059 & 1.278403 & -1.385652 \\
\hline 21 & 1 & -4.310161 & -0.081247 & -2.222626 \\
\hline 22 & 1 & -5.533828 & -1.316414 & 1.250275 \\
\hline 23 & 1 & -5.548931 & -1.851808 & $-0.43072 \epsilon$ \\
\hline 24 & 1 & -4.885931 & 0.950761 & 0.586485 \\
\hline 25 & 1 & -6.060186 & 0.522454 & -0.663827 \\
\hline 26 & 7 & -2.980391 & -0.626312 & -0.678865 \\
\hline 27 & 6 & 3.519927 & -0.726464 & -0.943594 \\
\hline 28 & 1 & 3.053047 & -0.388661 & -1.867610 \\
\hline 29 & 1 & 4.598408 & -0.771936 & -1.081653 \\
\hline 30 & 6 & -1.462948 & 1.160909 & 2.220130 \\
\hline
\end{tabular}




$\begin{array}{lcccc}31 & 1 & -2.401932 & 1.586162 & 1.871929 \\ 32 & 1 & -1.121083 & 1.688706 & 3.111696 \\ 33 & 1 & -1.595368 & 0.106896 & 2.466031 \\ 34 & 8 & -0.546398 & 1.321121 & 1.147056 \\ 35 & 6 & 0.666926 & 0.738917 & 1.310726 \\ 36 & 8 & 0.954285 & 0.170950 & 2.349387 \\ 37 & 6 & 1.489113 & 0.825691 & 0.118283 \\ 38 & 16 & 3.215279 & 0.609346 & 0.332744 \\ 39 & 16 & 0.980643 & 1.844090 & -1.256087 \\ 40 & 6 & 1.338388 & 3.525743 & -0.658100 \\ 41 & 1 & 1.100724 & 4.218448 & -1.465219 \\ 42 & 1 & 0.720687 & 3.752469 & 0.209517 \\ 43 & 1 & 2.393966 & 3.613842 & -0.403749\end{array}$


$\mathrm{TS}_{2}-\mathrm{II}_{\text {cis }}$

$\mathrm{HF}=-1586.990187$ hartrees

Imaginary Frequencies: 1 (-217.1535 1/cm)

Zero-point correction $=0.362476$ (Hartree $/$ Particle)

Temperature: 298.150 Kelvin. Pressure: 1.00000 Atm.

Sum of electronic and thermal Free Energies $=-1586.679273$ hartrees

Coordinates:

\begin{tabular}{ccccc} 
Center & \multicolumn{2}{c}{ Atomic } & \multicolumn{3}{c}{ Coordinates (Angstroms) } \\
Number & Number & X & $Y$ & $Z$ \\
----------------------------- \\
1 & 6 & 4.145215 & -2.643770 & 0.390100 \\
2 & 1 & 4.819976 & -1.922078 & 0.859475 \\
3 & 1 & 4.731136 & -3.201685 & -0.345059 \\
4 & 1 & 3.794268 & -3.336526 & 1.154485 \\
5 & 6 & 3.011174 & -1.924795 & -0.270657 \\
6 & 6 & 1.729497 & -2.097185 & 0.119811 \\
7 & 1 & 1.509861 & -2.768178 & 0.944475 \\
8 & 6 & 0.636386 & -1.359315 & -0.436836 \\
9 & 1 & 0.742437 & -0.989301 & -1.449798 \\
10 & 6 & -0.674002 & -1.484794 & 0.056529 \\
11 & 1 & -0.829055 & -1.917690 & 1.036943 \\
12 & 6 & -1.732279 & -0.981825 & -0.659664 \\
13 & 1 & -1.546559 & -0.576740 & -1.650548 \\
14 & 6 & -3.440735 & -1.320856 & 1.071659 \\
15 & 6 & -4.088709 & -0.394531 & -1.075653 \\
16 & 6 & -4.949171 & -1.065247 & 1.045901 \\
17 & 1 & -3.195044 & -2.365034 & 1.275846 \\
18 & 1 & -2.926940 & -0.698361 & 1.810544 \\
19 & 6 & -5.116769 & 0.013288 & -0.027967 \\
20 & 1 & -3.734629 & 0.435229 & -1.686238 \\
21 & 1 & -4.487410 & -1.168625 & -1.738625 \\
22 & 1 & -5.327435 & -0.760125 & 2.019730 \\
23 & 1 & -5.476657 & -1.973429 & 0.747981 \\
24 & 1 & -4.863420 & 0.994933 & 0.377778 \\
25 & 1 & -6.125581 & 0.058819 & -0.434446 \\
26 & 7 & -2.995993 & -0.936141 & -0.267094 \\
27 & 6 & 3.345160 & -0.874406 & -1.246720 \\
28 & 1 & 2.707352 & -0.844126 & -2.127001 \\
29 & 1 & 4.387541 & -0.904071 & -1.559132 \\
30 & 6 & -1.518608 & 2.481620 & -1.199192
\end{tabular}




$\begin{array}{lcccc}31 & 1 & -2.408112 & 2.685358 & -0.607380 \\ 32 & 1 & -1.761346 & 1.816983 & -2.029042 \\ 33 & 1 & -1.119914 & 3.411413 & -1.607660 \\ 34 & 8 & -0.587990 & 1.881477 & -0.307831 \\ 35 & 6 & 0.557080 & 1.420304 & -0.864098 \\ 36 & 8 & 0.725826 & 1.465091 & -2.073058 \\ 37 & 6 & 1.465000 & 0.811117 & 0.088897 \\ 38 & 16 & 3.159229 & 0.783814 & -0.375065 \\ 39 & 16 & 1.095415 & 0.842262 & 1.828866 \\ 40 & 6 & 1.391782 & 2.587061 & 2.267035 \\ 41 & 1 & 1.188728 & 2.693368 & 3.332767 \\ 42 & 1 & 2.428558 & 2.858759 & 2.071847 \\ 43 & 1 & 0.721089 & 3.234346 & 1.704147\end{array}$


5.5 Energies and coordinates for alternative pathways: path III-IV with s-cis-2b

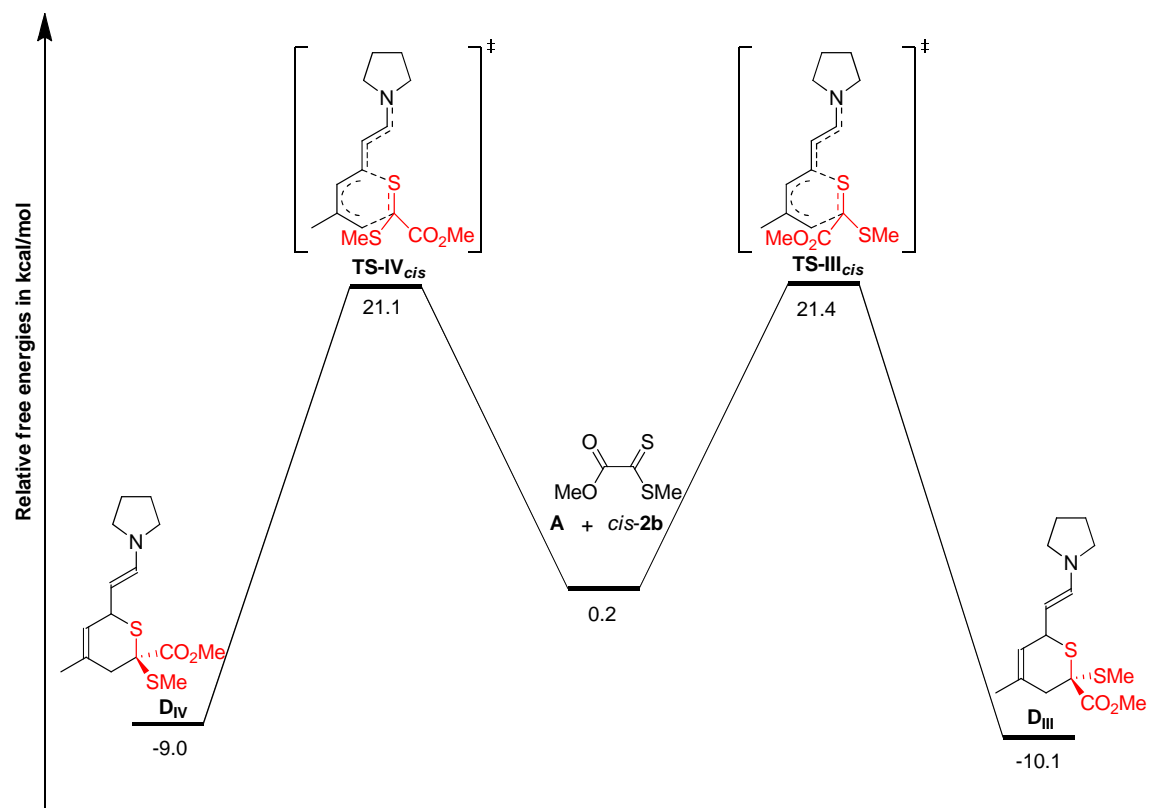

Figure S4: Energy profiles for pathways $\mathrm{III}_{\mathrm{c}}-\mathrm{IV}_{\mathrm{c}}$ : the formal cycloaddition of $\mathbf{A}$ and s-cis-2b (initial attack at carbon). Reported values in plain text are wB97xd/tzvp free energies in solvent (IEFPCM, $\mathrm{CHCl}_{3}$ ), relative to $\mathbf{A}+\mathbf{2 b}$. 
TS-III cis $_{\text {is }}$

$H F=-1586.9732948$ hartrees

Imaginary Frequencies: 1 (-450.3694 1/cm)

Zero-point correction $=0.361250$ (Hartree/Particle)

Temperature: 298.150 Kelvin. Pressure: 1.00000 Atm.

Sum of electronic and thermal Free Energies $=-1586.665772$ hartrees

Coordinates:

\begin{tabular}{cccccc} 
Center & \multicolumn{2}{c}{ Atomic } & \multicolumn{3}{c}{ Coordinates (Angstroms) } \\
Number & Number & X & $Y$ & $Z$ \\
----------------------------------- \\
1 & 6 & 2.313975 & -2.837829 & -0.954549 \\
2 & 1 & 2.456435 & -3.038244 & 0.111012 \\
3 & 1 & 3.292419 & -2.669171 & -1.404138 \\
4 & 1 & 1.875471 & -3.731344 & -1.402457 \\
5 & 6 & 1.408390 & -1.650541 & -1.136578 \\
6 & 6 & 0.062292 & -1.800201 & -0.770380 \\
7 & 1 & -0.232727 & -2.758541 & -0.351777 \\
8 & 6 & -0.904253 & -0.817550 & -0.845315 \\
9 & 1 & -0.664958 & 0.114002 & -1.346551 \\
10 & 6 & -2.234464 & -0.970067 & -0.403419 \\
11 & 1 & -2.518243 & -1.885688 & 0.101756 \\
12 & 6 & -3.143122 & 0.041840 & -0.571564 \\
13 & 1 & -2.822833 & 0.957762 & -1.060040 \\
14 & 6 & -5.041873 & -1.074535 & 0.524345 \\
15 & 6 & -5.329497 & 1.163145 & -0.372111 \\
16 & 6 & -6.504867 & -0.647296 & 0.648501 \\
17 & 1 & -4.918383 & -2.005154 & -0.033874 \\
18 & 1 & -4.567664 & -1.201430 & 1.502878 \\
19 & 6 & -6.433098 & 0.881709 & 0.641528 \\
20 & 1 & -4.814769 & 2.108377 & -0.200838 \\
21 & 1 & -5.724889 & 1.160273 & -1.392920 \\
22 & 1 & -6.973027 & -1.050232 & 1.544617 \\
23 & 1 & -7.070199 & -0.998723 & -0.216893 \\
24 & 1 & -6.137737 & 1.252330 & 1.625214 \\
25 & 1 & -7.375528 & 1.352348 & 0.366949 \\
26 & 7 & -4.412468 & 0.037979 & -0.182328 \\
27 & 6 & 1.956771 & -0.427976 & -1.496993 \\
28 & 1 & 1.319300 & 0.362224 & -1.867315 \\
29 & 1 & 2.974721 & -0.401247 & -1.862612 \\
30 & 6 & 5.437704 & -1.021996 & 0.828462
\end{tabular}




$\begin{array}{lcccc}31 & 1 & 6.339954 & -0.753445 & 0.286612 \\ 32 & 1 & 5.158110 & -2.053304 & 0.612611 \\ 33 & 1 & 5.591320 & -0.905663 & 1.900863 \\ 34 & 8 & 4.428390 & -0.122851 & 0.366888 \\ 35 & 6 & 3.211852 & -0.269031 & 0.903471 \\ 36 & 8 & 2.956575 & -1.100256 & 1.734456 \\ 37 & 6 & 2.216228 & 0.716766 & 0.326705 \\ 38 & 16 & 0.708458 & 0.847555 & 1.070221 \\ 39 & 16 & 3.056769 & 2.147064 & -0.408629 \\ 40 & 6 & 1.673264 & 3.259030 & -0.747830 \\ 41 & 1 & 2.116748 & 4.140157 & -1.210894 \\ 42 & 1 & 0.964397 & 2.804087 & -1.438902 \\ 43 & 1 & 1.160193 & 3.532917 & 0.171129\end{array}$


TS-IV cis

$\mathrm{HF}=-1586.973058$ hartrees

Imaginary Frequencies: $1(-420.3331$ 1/cm)

Zero-point correction $=0.360762$ (Hartree/Particle)

Temperature: 298.150 Kelvin. Pressure: 1.00000 Atm.

Sum of electronic and thermal Free Energies $=-1586.666245$ hartrees

Coordinates:

\begin{tabular}{cccccc} 
Center & \multicolumn{2}{c}{ Atomic } & \multicolumn{3}{c}{ Coordinates (Angstroms) } \\
Number & Number & X & $Y$ & $Z$ \\
------------------------------ \\
1 & 6 & -2.150779 & 2.886923 & -1.420982 \\
2 & 1 & -2.376065 & 3.146132 & -2.458772 \\
3 & 1 & -1.632492 & 3.732244 & -0.967607 \\
4 & 1 & -3.103137 & 2.739251 & -0.907566 \\
5 & 6 & -1.329180 & 1.626982 & -1.370452 \\
6 & 6 & 0.034510 & 1.724154 & -1.055871 \\
7 & 1 & 0.424104 & 2.712170 & -0.827198 \\
8 & 6 & 0.914043 & 0.661680 & -0.965757 \\
9 & 1 & 0.577533 & -0.320979 & -1.276660 \\
10 & 6 & 2.267972 & 0.773761 & -0.590491 \\
11 & 1 & 2.652808 & 1.740160 & -0.286615 \\
12 & 6 & 3.073379 & -0.335450 & -0.565398 \\
13 & 1 & 2.649708 & -1.296539 & -0.842547 \\
14 & 6 & 5.115073 & 0.798881 & 0.204979 \\
15 & 6 & 5.157553 & -1.597503 & -0.190520 \\
16 & 6 & 6.542779 & 0.272398 & 0.354795 \\
17 & 1 & 5.034215 & 1.594419 & -0.539071 \\
18 & 1 & 4.714859 & 1.177495 & 1.151175 \\
19 & 6 & 6.347524 & -1.210209 & 0.680857 \\
20 & 1 & 4.577585 & -2.427158 & 0.213015 \\
21 & 1 & 5.480074 & -1.856035 & -1.204081 \\
22 & 1 & 7.098400 & 0.809358 & 1.121445 \\
23 & 1 & 7.078456 & 0.379970 & -0.590377 \\
24 & 1 & 6.094291 & -1.336957 & 1.735294 \\
25 & 1 & 7.228592 & -1.812569 & 0.466199 \\
26 & 7 & 4.353304 & -0.373932 & -0.215343 \\
27 & 6 & -1.977145 & 0.411885 & -1.533816 \\
28 & 1 & -1.415840 & -0.471129 & -1.804051 \\
29 & 1 & -3.011405 & 0.426706 & -1.858845 \\
30 & 6 & -4.960754 & -2.510604 & -0.701614 \\
& & & &
\end{tabular}




$\begin{array}{lcccc}31 & 1 & -6.010049 & -2.247429 & -0.605720 \\ 32 & 1 & -4.753273 & -3.439965 & -0.172283 \\ 33 & 1 & -4.692085 & -2.624476 & -1.751822 \\ 34 & 8 & -4.236344 & -1.427200 & -0.115916 \\ 35 & 6 & -2.906832 & -1.547336 & -0.083507 \\ 36 & 8 & -2.322869 & -2.510134 & -0.508536 \\ 37 & 6 & -2.249266 & -0.327281 & 0.530932 \\ 38 & 16 & -0.712797 & -0.508243 & 1.197847 \\ 39 & 16 & -3.466447 & 0.809697 & 1.242918 \\ 40 & 6 & -2.399999 & 2.016450 & 2.067272 \\ 41 & 1 & -1.877518 & 1.565476 & 2.907392 \\ 42 & 1 & -1.675587 & 2.431249 & 1.369572 \\ 43 & 1 & -3.067959 & 2.803152 & 2.417347\end{array}$


<smiles>C=S</smiles>

$\mathbf{E}$

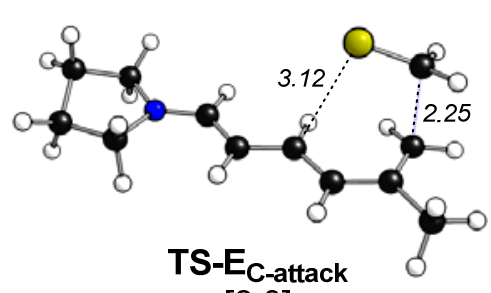

$[0.0]$

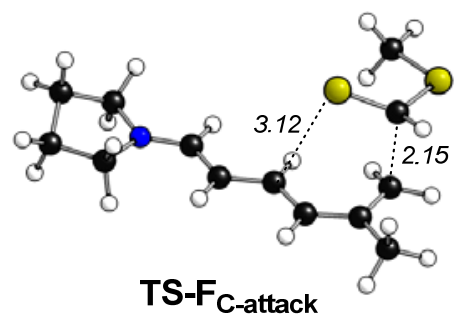

$[0.0]$

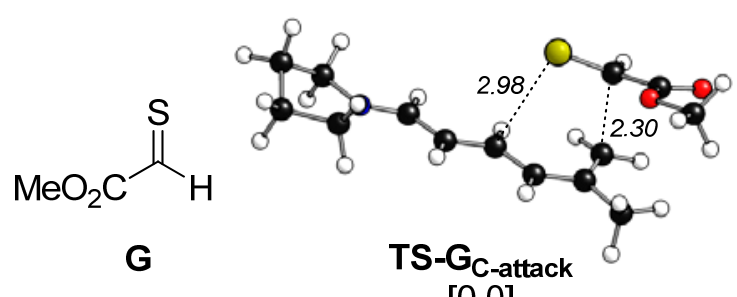

$[0.0]$

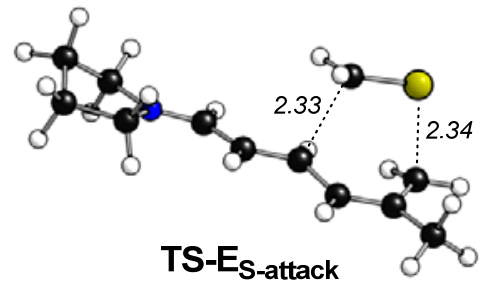

$+7.4$

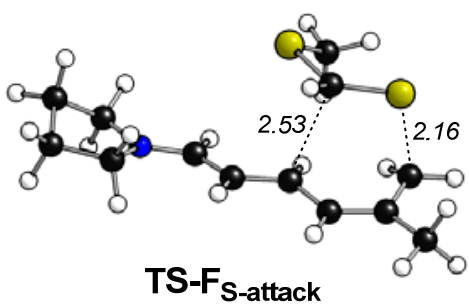

$+4.7$

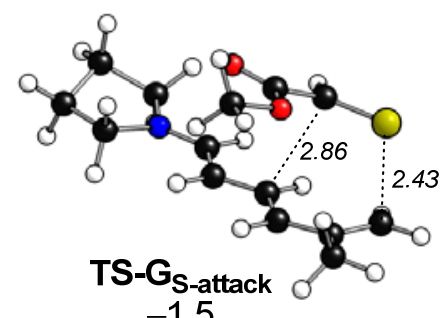

Figure S5: Activation energies for the addition of $\mathbf{A}$ to thiocarbonyls E,F,G via the two possible pathways (initial attack at sulfur, S-attack pathway; or, initial attack at carbon, C-attack pathway). Reported values in plain text are wB97xd/tzvp free energies in solvent (IEFPCM, $\mathrm{CHCl}_{3}$ ). Values in italics are bond distances in Ångstrøm. 
TS-E $\mathrm{E}_{\mathrm{C} \text {-attack }}$

$\mathrm{HF}=-921.5777942$ hartrees

Imaginary Frequencies: 1 (-342.8046 1/cm)

Zero-point correction $=0.287179$ (Hartree/Particle)

Temperature: 298.150 Kelvin. Pressure: 1.00000 Atm.

Sum of electronic and thermal Free Energies $=-921.335968$ hartrees

Coordinates:

\begin{tabular}{|c|c|c|c|c|}
\hline Center & & & oordinates (A & Angstroms \\
\hline Number & & & $x$ & Z \\
\hline 1 & 6 & -4.253381 & -1.883085 & -0.488239 \\
\hline 2 & 1 & -4.236485 & -1.557099 & -1.532800 \\
\hline 3 & 1 & -5.263675 & -1.750423 & -0.102509 \\
\hline 4 & 1 & -4.015519 & -2.948980 & -0.481156 \\
\hline 5 & 6 & -3.243083 & -1.103584 & 0.308941 \\
\hline 6 & 6 & -1.878714 & -1.295103 & -0.042063 \\
\hline 7 & 1 & -1.674068 & -2.032227 & -0.813982 \\
\hline 8 & 6 & -0.820421 & -0.584990 & 0.451876 \\
\hline 9 & 1 & -0.997812 & 0.137085 & 1.242947 \\
\hline 10 & 6 & 0.532411 & -0.755697 & 0.053155 \\
\hline 11 & 1 & 0.757838 & -1.471944 & -0.728683 \\
\hline 12 & 6 & 1.526799 & -0.009850 & 0.615488 \\
\hline 13 & 1 & 1.270413 & 0.714696 & 1.383356 \\
\hline 14 & 6 & 3.380666 & -0.926308 & -0.718173 \\
\hline 15 & 6 & 3.841686 & 0.789705 & 0.934603 \\
\hline 16 & 6 & 4.888945 & -0.694564 & -0.618500 \\
\hline 17 & 1 & 3.103113 & -1.967853 & -0.537461 \\
\hline 18 & 1 & 2.984376 & -0.638093 & -1.698101 \\
\hline 19 & 6 & 4.998642 & 0.725712 & -0.057393 \\
\hline 20 & 1 & 3.457985 & 1.799155 & 1.084592 \\
\hline 21 & 1 & 4.136364 & 0.381404 & 1.907310 \\
\hline 22 & 1 & 5.386562 & -0.814869 & -1.579183 \\
\hline 23 & 1 & 5.328490 & -1.407369 & 0.082222 \\
\hline 24 & 1 & 4.847689 & 1.459732 & -0.851596 \\
\hline 25 & 1 & 5.959602 & 0.924205 & 0.414419 \\
\hline 26 & 7 & 2.825897 & -0.057343 & 0.312034 \\
\hline 27 & 6 & -3.675276 & ; -0.157269 & 1.199366 \\
\hline 28 & 1 & -2.994050 & 0.312932 & 1.895293 \\
\hline 29 & 1 & -4.724679 & -0.112363 & 1.463030 \\
\hline 30 & 6 & -3.656952 & 1.663167 & -0.11932 \\
\hline
\end{tabular}


$\begin{array}{llllll}31 & 16 & -2.157259 & 1.955660 & -0.780199\end{array}$

$\begin{array}{llllll}32 & 1 & -3.977235 & 2.195595 & 0.770174\end{array}$

$\begin{array}{llllll}33 & 1 & -4.461970 & 1.278449 & -0.737378\end{array}$ 
TS-E S-attack $_{\text {}}$

$\mathrm{HF}=-921.5684276$ hartrees

Imaginary Frequencies: 1 (-394.0095 1/cm)

Zero-point correction $=0.287789$ (Hartree/Particle)

Temperature: 298.150 Kelvin. Pressure: 1.00000 Atm.

Sum of electronic and thermal Free Energies $=-921.324139$ hartrees

Coordinates:

\begin{tabular}{|c|c|c|c|c|}
\hline \multirow{2}{*}{$\begin{array}{l}\text { Center } \\
\text { Number }\end{array}$} & \multicolumn{2}{|c|}{ Atomic } & \multicolumn{2}{|c|}{ Coordinates (Angstroms) } \\
\hline & & ber & $X \quad Y$ & Z \\
\hline 1 & 6 & -4.288460 & -1.891229 & -0.293162 \\
\hline 2 & 1 & -3.909570 & -2.900899 & -0.457891 \\
\hline 3 & 1 & -4.541025 & -1.468846 & -1.270659 \\
\hline 4 & 1 & -5.208532 & -1.959864 & 0.287693 \\
\hline 5 & 6 & -3.257538 & -1.030932 & 0.391755 \\
\hline 6 & 6 & -1.909324 & -1.240126 & 0.090483 \\
\hline 7 & 1 & -1.667942 & -2.063662 & -0.577251 \\
\hline 8 & 6 & -0.873970 & -0.379624 & 0.428058 \\
\hline 9 & 1 & -1.027092 & 0.314987 & 1.246408 \\
\hline 10 & & 0.493745 & -0.609428 & 0.046889 \\
\hline 11 & 1 & 0.692023 & -1.356652 & -0.713282 \\
\hline 12 & 6 & & 0.104678 & 0.591143 \\
\hline 13 & 1 & 1.286029 & 0.853744 & 1.345912 \\
\hline 14 & 6 & 3.332126 & -0.920023 & -0.711002 \\
\hline 15 & 6 & 3.867783 & 0.785790 & 0.926231 \\
\hline 16 & 6 & 4.850662 & -0.764026 & -0.604525 \\
\hline 17 & 1 & 3.002880 & -1.943872 & -0.510899 \\
\hline 18 & 1 & 2.957404 & -0.638703 & -1.702404 \\
\hline 19 & 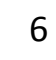 & 5.030181 & 0.652888 & -0.052855 \\
\hline 20 & 1 & 3.543601 & 1.817763 & 1.068036 \\
\hline 21 & 1 & 4.130958 & 0.369211 & 1.905373 \\
\hline 22 & 1 & 5.348054 & -0.917130 & -1.560810 \\
\hline 23 & 1 & 5.249412 & -1.492507 & 0.104505 \\
\hline 24 & 1 & 4.925344 & 1.387737 & -0.853895 \\
\hline 25 & 1 & 5.996337 & 0.804594 & 0.426060 \\
\hline 26 & 7 & 2.816275 & -0.002855 & 0.293002 \\
\hline 27 & 6 & -3.662712 & 0.095268 & 1.089495 \\
\hline 28 & 1 & -3.008071 & 0.568325 & 1.807086 \\
\hline 29 & 1 & -4.719841 & 0.255698 & 1.266312 \\
\hline 30 & 6 & -1.615465 & 1.427348 & -0.851083 \\
\hline
\end{tabular}


$\begin{array}{lllll}31 & 16 & -3.215997 & 1.755149 & -0.501532\end{array}$

$\begin{array}{llllll}32 & 1 & -0.824665 & 1.984249 & -0.363225\end{array}$

$\begin{array}{llllll}33 & 1 & -1.352488 & 0.939925 & -1.782135\end{array}$ 
TS-F C-attack $_{\text {}}$

$H F=-1359.0892375$ hartrees

Imaginary Frequencies: 1 (-508.9094 1/cm)

Zero-point correction $=0.317831$ (Hartree/Particle)

Temperature: 298.150 Kelvin. Pressure: 1.00000 Atm.

Sum of electronic and thermal Free Energies $=-1358.820413$ hartrees

Coordinates:

\begin{tabular}{|c|c|c|c|c|}
\hline \multirow{3}{*}{$\begin{array}{l}\text { Center } \\
\text { Number } \\
1\end{array}$} & \multirow{2}{*}{\multicolumn{2}{|c|}{$\begin{array}{l}\text { Atomic } \\
\text { Number }\end{array}$}} & \multicolumn{2}{|c|}{ Coordinates (Angstroms) } \\
\hline & & & $X \quad Y$ & Z \\
\hline & 6 & -3.014222 & 3.194538 & 0.043365 \\
\hline 2 & 1 & -3.150245 & 3.080945 & 1.124299 \\
\hline 3 & 1 & -4.000238 & 3.247619 & -0.417237 \\
\hline 4 & 1 & -2.499272 & 4.142849 & -0.118034 \\
\hline 5 & 6 & -2.210167 & 2.038309 & -0.483061 \\
\hline 6 & 6 & -0.821299 & 2.056967 & -0.235251 \\
\hline 7 & 1 & -0.420014 & 2.934328 & 0.264586 \\
\hline 8 & 6 & 0.045932 & 1.026662 & -0.502424 \\
\hline 9 & 1 & -0.326860 & 0.156008 & -1.031948 \\
\hline 10 & 6 & 1.425578 & 1.026959 & -0.192912 \\
\hline 11 & 1 & 1.844775 & 1.877211 & 0.332457 \\
\hline 12 & 6 & 2.213085 & -0.041841 & -0.523697 \\
\hline 13 & 1 & 1.761018 & -0.889815 & -1.030524 \\
\hline 14 & 6 & 4.312902 & 0.832519 & 0.412693 \\
\hline 15 & 6 & 4.300635 & -1.350410 & -0.651000 \\
\hline 16 & 6 & 5.740514 & 0.293473 & 0.317910 \\
\hline 17 & 1 & 4.197446 & 1.808212 & -0.065099 \\
\hline 18 & 1 & 3.975570 & 0.922940 & 1.450627 \\
\hline 19 & 6 & 5.547276 & $5-1.221949$ & 0.218093 \\
\hline 20 & 1 & 3.736798 & $3-2.264084$ & -0.462216 \\
\hline 21 & 1 & 4.556823 & $3-1.312332$ & -1.714891 \\
\hline 22 & 1 & 6.348267 & 0.591491 & 1.170448 \\
\hline 23 & 1 & 6.220334 & 0.669741 & -0.587706 \\
\hline 24 & 1 & 5.357325 & -1.645988 & 1.206080 \\
\hline 25 & 1 & 6.405991 & -1.732809 & -0.214159 \\
\hline 26 & 7 & 3.514111 & -0.174545 & -0.277697 \\
\hline 27 & 6 & -2.875478 & 0.945064 & -1.000510 \\
\hline 28 & 1 & -2.337383 & 0.189542 & -1.556474 \\
\hline 29 & 1 & -3.918848 & 1.052545 & -1.266659 \\
\hline 30 & 6 & -3.177973 & -0.368149 & 0.680860 \\
\hline
\end{tabular}




$\begin{array}{lcccc}31 & 16 & -1.715258 & -0.609777 & 1.483865 \\ 32 & 16 & -4.095072 & -1.666226 & -0.149367 \\ 33 & 6 & -2.793467 & -2.821001 & -0.643195 \\ 34 & 1 & -3.295871 & -3.652087 & -1.135884 \\ 35 & 1 & -2.102503 & -2.349596 & -1.341679 \\ 36 & 1 & -2.247741 & -3.175231 & 0.228469 \\ 37 & 1 & -3.898778 & 0.319515 & 1.112233\end{array}$


TS-F $F_{\text {-attack }}$

$\mathrm{HF}=-1359.0816983$ hartrees

Imaginary Frequencies: 1 (-366.4687 1/cm)

Zero-point correction $=0.316898$ (Hartree/Particle)

Temperature: 298.150 Kelvin. Pressure: 1.00000 Atm.

Sum of electronic and thermal Free Energies $=-1358.812975$ hartrees

Coordinates:

\begin{tabular}{ccccc} 
Center & Atomic & \multicolumn{3}{c}{ Coordinates (Angstroms) } \\
Number & Number & X & $Y$ & Z \\
\hline-------------------------- & \\
1 & 6 & -4.071460 & -2.601075 & -0.088985 \\
2 & 1 & -3.613700 & -3.544166 & -0.388482 \\
3 & 1 & -4.567749 & -2.174260 & -0.967056 \\
4 & 1 & -4.844421 & -2.813845 & 0.652099 \\
5 & 6 & -3.047351 & -1.635144 & 0.441675 \\
6 & 6 & -1.699158 & -1.871850 & 0.219864 \\
7 & 1 & -1.425572 & -2.786617 & -0.299992 \\
8 & 6 & -0.679048 & -0.964680 & 0.491130 \\
9 & 1 & -0.879261 & -0.160608 & 1.188557 \\
10 & 6 & 0.687553 & -1.181736 & 0.164604 \\
11 & 1 & 0.936269 & -1.991474 & -0.512484 \\
12 & 6 & 1.663924 & -0.356591 & 0.639476 \\
13 & 1 & 1.392289 & 0.439513 & 1.324184 \\
14 & 6 & 3.534543 & -1.353029 & -0.612994 \\
15 & 6 & 3.955729 & 0.537946 & 0.845883 \\
16 & 6 & 5.032908 & -1.048871 & -0.580742 \\
17 & 1 & 3.310158 & -2.386002 & -0.334087 \\
18 & 1 & 3.101050 & -1.173294 & -1.604165 \\
19 & 6 & 5.088140 & 0.424998 & -0.170569 \\
20 & 1 & 3.523224 & 1.538199 & 0.897554 \\
21 & 1 & 4.298372 & 0.257610 & 1.848164 \\
22 & 1 & 5.512602 & -1.244470 & -1.538297 \\
23 & 1 & 5.522592 & -1.666143 & 0.175364 \\
24 & 1 & 4.878615 & 1.064008 & -1.031008 \\
25 & 1 & 6.050844 & 0.716497 & 0.246622 \\
26 & 7 & 2.970340 & -0.418630 & 0.349897 \\
27 & 6 & -3.490772 & -0.382622 & 0.905978 \\
28 & 1 & -2.885568 & 0.169464 & 1.612606 \\
29 & 1 & -4.556030 & -0.257190 & 1.071765 \\
30 & 6 & -1.507571 & 0.909101 & -0.999504 \\
& & & &
\end{tabular}




$\begin{array}{lcccl}31 & 16 & -3.173615 & 0.913186 & -0.799158 \\ 32 & 16 & -0.388348 & 2.188296 & -0.475315 \\ 33 & 6 & -1.202757 & 2.852614 & 1.000509 \\ 34 & 1 & -0.655661 & 3.749794 & 1.284395 \\ 35 & 1 & -2.237082 & 3.108304 & 0.775863 \\ 36 & 1 & -1.172463 & 2.137822 & 1.822668 \\ 37 & 1 & -1.077556 & 0.349190 & -1.819935\end{array}$


TS-G $\mathbf{G}_{\text {-attack }}$

$\mathrm{HF}=-1149.4690305$ hartrees

Imaginary Frequencies: 1 (-283.2636 1/cm)

Zero-point correction $=0.332005$ (Hartree/Particle)

Temperature: 298.150 Kelvin. Pressure: 1.00000 Atm.

Sum of electronic and thermal Free Energies $=-1149.187018$ hartrees

Coordinates:

\begin{tabular}{|c|c|c|c|c|}
\hline \multirow{3}{*}{$\begin{array}{l}\text { Center } \\
\text { Number } \\
1\end{array}$} & \multirow{2}{*}{\multicolumn{2}{|c|}{$\begin{array}{l}\text { Atomic } \\
\text { Number }\end{array}$}} & \multicolumn{2}{|c|}{ Coordinates (Angstroms } \\
\hline & & & $x$ & Z \\
\hline & 6 & -3.008782 & 2.397982 & 0.019697 \\
\hline 2 & 1 & -2.980669 & 2.221434 & 1.096455 \\
\hline 3 & 1 & -4.014172 & 2.184527 & -0.344035 \\
\hline 4 & 1 & -2.810504 & 3.460358 & -0.143824 \\
\hline 5 & 6 & -1.976122 & 1.561322 & -0.687441 \\
\hline 6 & 6 & -0.677014 & 1.529373 & -0.125878 \\
\hline 7 & 1 & -0.530444 & 2.080365 & 0.799078 \\
\hline 8 & 6 & 0.397403 & 0.832368 & -0.622172 \\
\hline 9 & 1 & 0.297441 & 0.333334 & -1.580668 \\
\hline 10 & 6 & 1.684383 & 0.799224 & -0.030190 \\
\hline 11 & 1 & 1.834567 & 1.295501 & 0.921504 \\
\hline 12 & 6 & & 0.126440 & -0.632265 \\
\hline 13 & 1 & 2.522820 & -0.384117 & -1.572790 \\
\hline 14 & 6 & 4.429601 & 0.609185 & 1.062072 \\
\hline 15 & 6 & 5.006369 & -0.741439 & -0.871010 \\
\hline 16 & 6 & 5.933762 & 0.332945 & 1.051232 \\
\hline 17 & 1 & 4.196996 & 1.676512 & 1.088416 \\
\hline 18 & 1 & 3.932195 & 0.133981 & 1.914336 \\
\hline 19 & 6 & 6.056156 & -0.944179 & 0.216582 \\
\hline 20 & 1 & 4.608535 & -1.676131 & -1.266774 \\
\hline 21 & 1 & 5.411073 & -0.155722 & -1.702995 \\
\hline 22 & 1 & 6.339521 & 0.227374 & 2.055820 \\
\hline 23 & 1 & 6.458553 & 1.153956 & 0.558423 \\
\hline 24 & 1 & 5.801045 & -1.817068 & 0.821070 \\
\hline 25 & 1 & 7.053012 & -1.091899 & -0.195445 \\
\hline 26 & 7 & & 0.010881 & -0.181797 \\
\hline 27 & 6 & -2.350446 & 0.783836 & -1.754499 \\
\hline 28 & 1 & -1.615186 & 0.328534 & -2.402877 \\
\hline 29 & 1 & -3.348433 & 0.873839 & -2.164909 \\
\hline 30 & 6 & -4.778473 & -0.160584 & $4 \quad 1.96743$ \\
\hline
\end{tabular}




$\begin{array}{lcccc}31 & 1 & -4.435008 & 0.100367 & 2.964391 \\ 32 & 1 & -5.483586 & -0.990056 & 2.013739 \\ 33 & 1 & -5.262260 & 0.696479 & 1.497822 \\ 34 & 8 & -3.606721 & -0.532939 & 1.241992 \\ 35 & 6 & -3.793335 & -0.917715 & -0.017372 \\ 36 & 8 & -4.884384 & -0.985464 & -0.538783 \\ 37 & 6 & -2.533899 & -1.268141 & -0.740241 \\ 38 & 16 & -1.100196 & -1.662385 & 0.000961 \\ 39 & 1 & -2.747965 & -1.626046 & -1.740914\end{array}$


TS-G $\mathbf{G}_{\text {-attack }}$

$\mathrm{HF}=-1149.4717753$ hartrees

Imaginary Frequencies: 1 (-166.5992 1/cm)

Zero-point correction $=0.331601$ (Hartree/Particle)

Temperature: 298.150 Kelvin. Pressure: 1.00000 Atm.

Sum of electronic and thermal Free Energies $=-1149.189462$ hartrees

Coordinates:

\begin{tabular}{|c|c|c|c|c|}
\hline \multirow{2}{*}{$\begin{array}{l}\text { Center } \\
\text { Number }\end{array}$} & \multicolumn{2}{|c|}{ Atomic } & \multicolumn{2}{|c|}{ Coordinates (Angstroms) } \\
\hline & & & $\mathrm{Y}$ & Z \\
\hline 1 & 6 & -3.856250 & -0.843911 & -1.823486 \\
\hline 2 & 1 & -3.607849 & -0.965385 & -2.879737 \\
\hline 3 & 1 & -3.954521 & 0.226855 & -1.626337 \\
\hline 4 & 1 & -4.820300 & -1.315945 & -1.637379 \\
\hline 5 & 6 & -2.774598 & -1.417815 & -0.949041 \\
\hline 6 & 6 & -1.445677 & -1.028991 & -1.243594 \\
\hline 7 & 1 & -1.304344 & -0.406717 & -2.123400 \\
\hline 8 & 6 & -0.340356 & -1.285026 & -0.470735 \\
\hline 9 & 1 & -0.447199 & -1.928412 & 0.397663 \\
\hline 10 & 6 & 0.961048 & -0.807720 & -0.744739 \\
\hline 11 & 1 & 1.110502 & -0.167968 & -1.606335 \\
\hline 12 & 6 & & & 0.109530 \\
\hline 13 & 1 & 1.814220 & -1.691583 & 0.981817 \\
\hline 14 & 6 & 3.685518 & 0.276544 & -1.062432 \\
\hline 15 & 6 & 4.299881 & -0.893760 & 0.974075 \\
\hline 16 & 6 & 5.183511 & 0.436578 & -0.803787 \\
\hline 17 & 1 & 3.470146 & -0.151099 & -2.044301 \\
\hline 18 & 1 & 3.150710 & 1.228707 & -0.980961 \\
\hline 19 & 6 & 5.311215 & 0.216172 & 0.705991 \\
\hline 20 & 1 & 3.896664 & -0.874305 & 1.986639 \\
\hline 21 & 1 & 4.741221 & -1.879311 & 0.793388 \\
\hline 22 & 1 & 5.553671 & 1.408003 & -1.126738 \\
\hline 23 & 1 & 5.739254 & -0.333611 & -1.342664 \\
\hline 24 & 1 & 5.021694 & 1.120424 & 1.244965 \\
\hline 25 & 1 & 6.318785 & -0.059025 & 1.013358 \\
\hline 26 & 7 & 3.244053 & -0.622634 & -0.001561 \\
\hline 27 & 6 & -3.115572 & -2.133372 & 0.173659 \\
\hline 28 & 1 & -2.381617 & -2.699159 & 0.729442 \\
\hline 29 & 1 & -4.147218 & -2.422793 & 0.330426 \\
\hline 30 & 6 & -1.962156 & 3.028073 & -0.95924 \\
\hline
\end{tabular}




$\begin{array}{lcccc}31 & 1 & -2.898238 & 3.253860 & -1.463340 \\ 32 & 1 & -1.206665 & 2.729409 & -1.687805 \\ 33 & 1 & -1.606565 & 3.904803 & -0.417236 \\ 34 & 8 & -2.251806 & 1.956815 & -0.068881 \\ 35 & 6 & -1.226441 & 1.516499 & 0.677204 \\ 36 & 8 & -0.127525 & 2.037105 & 0.649051 \\ 37 & 6 & -1.541574 & 0.368303 & 1.531137 \\ 38 & 16 & -3.030586 & -0.295573 & 1.754990 \\ 39 & 1 & -0.675121 & -0.010434 & 2.061060\end{array}$




\subsection{Facial shielding provided by catalyst 3: Structures and energies}

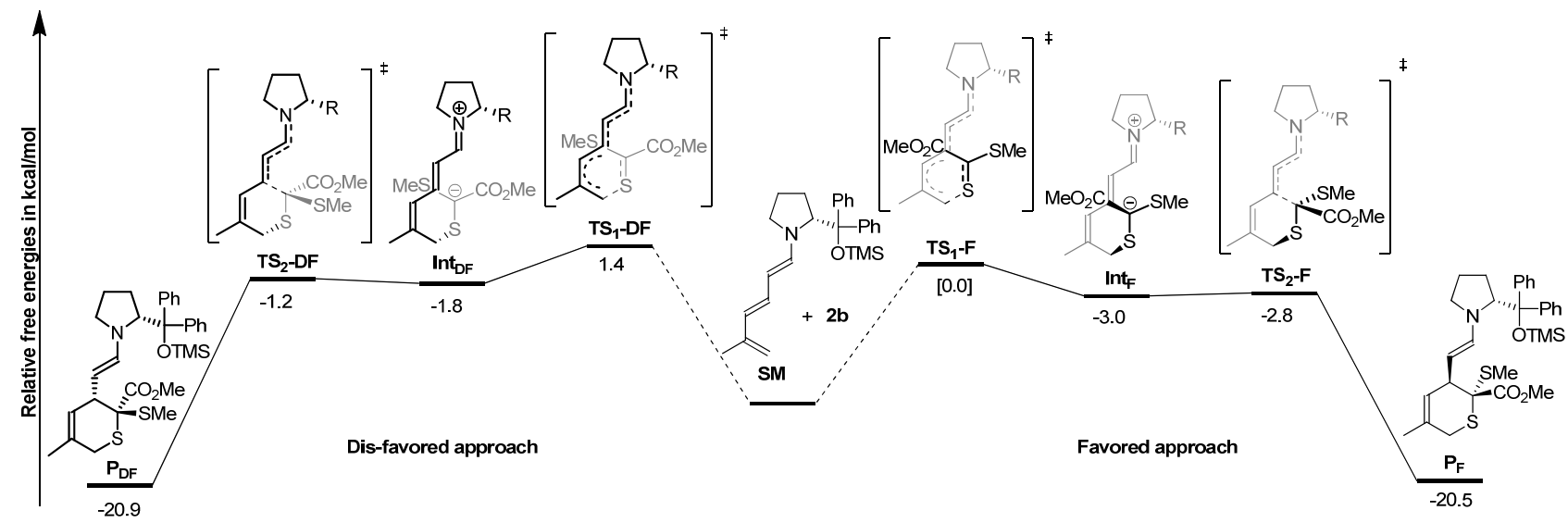

Figure S6: Energy profiles for the favored and the disfavored approach of dithioester $\mathbf{2} \mathbf{b}$ to the catalystbound trienamine SM. Reported values in plain text are wB97xd/cc-pVDZ/IEFPCM//wB97xd/6$31 \mathrm{G}(\mathrm{d}) / \mathrm{IEFPCM}$ free energies. $\mathrm{R}=-\mathrm{C}(\mathrm{Ph})_{2} \mathrm{OTMS}$.

Difference in activation energies with different methods
Method
$\Delta \Delta \mathrm{G}_{(\mathrm{TS1} 1 \mathrm{DF}-\mathrm{TS1} 1 \mathrm{~F})}^{\ddagger}$

\begin{tabular}{|l|c|}
\hline wB97xd/6-31G(d) in $\mathrm{CHCl}_{3}$ & 1.6 \\
\hline wB97xd/tzvp//wB97xd/6-31G(d) in $\mathrm{CHCl}_{3}$ & 1.6 \\
\hline M062X/tzvp//wB97xd/6-31G(d) in $\mathrm{CHCl}_{3}$ & 1.8 \\
\hline wB97xd/cc-pVDZ//wB97xd/6-31G(d) in $\mathrm{CHCl}_{3}$ & 1.4 \\
\hline wB97xd/cc-pVTZ//wB97xd/6-31G(d) in $\mathrm{CHCl}_{3}$ & 1.6 \\
\hline wB97xd/cc-pVQZ//wB97xd/6-31G(d) in $\mathrm{CHCl}_{3}$ & 1.7 \\
\hline
\end{tabular}

* All values are free energies at $298.15 \mathrm{~K}$.

Experimental result: When dithioester $\mathbf{2 b}$ is reacted with dienal $\mathbf{1 a}$ at $25^{\circ} \mathrm{C}$ in the presence of catalyst $\mathbf{3}$ (20 mol\%) and $\mathrm{PhCO}_{2} \mathrm{H}\left(20\right.$ mol\%) in $\mathrm{CHCl}_{3}$, the desired cycloadduct was formed in 84\% yield, 79:21 dr and 79\% ee. 
$\mathrm{TS}_{1}-\mathrm{F}$

$\mathrm{HF}=-2571.8061396$ hartrees

Imaginary Frequencies: 1 (-246.7239 1/cm)

Zero-point correction $=0.665721$ (Hartree/Particle)

Temperature: 298.150 Kelvin. Pressure: 1.00000 Atm.

Thermal correction to Gibbs Free Energy= 0.591201

Sum of electronic and thermal Free Energies $=-2571.214939$ hartrees

Single Point Energy (wB97xd/cc-pVDZ in chloroform) Single Point Energy (wB97xd/cc-pVTZ in chloroform) Single Point Energy (wB97xd/cc-pVQZ in chloroform) Single Point Energy (wB97xd/tzvp in chloroform) Single Point Energy (M062x/tzvp in chloroform)

Coordinates:

$\begin{array}{lccc}6 & -3.931955000 & 0.873385000 & -1.765165000 \\ 6 & -5.061222000 & 0.062160000 & -1.984925000 \\ 6 & -5.057787000 & -1.315105000 & -1.758247000 \\ 6 & -2.705644000 & 0.416808000 & -1.318334000 \\ 6 & -1.641236000 & 1.224577000 & -0.877041000 \\ 6 & -0.539087000 & 0.619670000 & -0.318703000 \\ 7 & 0.437108000 & 1.219162000 & 0.364428000 \\ 6 & 1.529654000 & 0.473905000 & 0.982295000 \\ 6 & 1.899619000 & 1.348101000 & 2.193088000 \\ 6 & 1.478884000 & 2.777211000 & 1.812538000 \\ 6 & 0.307132000 & 2.593272000 & 0.844027000 \\ 1 & -4.071421000 & 1.951218000 & -1.842585000 \\ 1 & -5.940641000 & -1.888688000 & -2.028904000 \\ 1 & -1.764021000 & 2.303211000 & -0.845278000 \\ 1 & -0.442226000 & -0.455281000 & -0.402342000 \\ 1 & 1.130976000 & -0.494915000 & 1.299197000 \\ 1 & 2.959940000 & 1.285254000 & 2.446919000 \\ 1 & 1.328377000 & 1.002819000 & 3.060116000 \\ 1 & 2.300019000 & 3.299680000 & 1.317418000 \\ 1 & 1.186401000 & 3.360390000 & 2.688513000 \\ 1 & 0.348687000 & 3.299939000 & 0.008304000 \\ 1 & -0.668970000 & 2.690109000 & 1.335680000 \\ 6 & 2.686116000 & 0.176774000 & -0.034239000 \\ 8 & 2.100135000 & -0.471164000 & -1.150804000 \\ 14 & 1.734168000 & -2.072373000 & -1.511727000 \\ 6 & 3.235727000 & -3.042000000 & -2.083243000 \\ 1 & 2.904345000 & -3.962552000 & -2.579384000 \\ 1 & 3.887844000 & -3.319626000 & -1.249778000 \\ 1 & 3.830521000 & -2.467817000 & -2.801797000 \\ 6 & 0.516714000 & -1.904773000 & -2.928797000 \\ 1 & -0.338043000 & -1.275784000 & -2.657118000 \\ 1 & 0.134543000 & -2.886366000 & -3.232116000 \\ 1 & 0.997996000 & -1.448042000 & -3.800601000 \\ 6 & 0.941251000 & -2.975585000 & -0.064482000\end{array}$

$\mathrm{HF}=-2571.937123$ hartrees $\mathrm{HF}=-2572.397864$ hartrees $\mathrm{HF}=-2572.525082$ hartrees $\mathrm{HF}=-2572.33557$ hartrees $\mathrm{HF}=-2572.132897$ hartrees 


$\begin{array}{llll}1 & 0.686682000 & -3.995352000 & -0.379319000 \\ 1 & 0.018393000 & -2.498445000 & 0.284388000 \\ 1 & 1.627483000 & -3.054815000 & 0.785489000 \\ 6 & 3.773146000 & -0.719947000 & 0.571594000 \\ 6 & 4.932017000 & -0.951689000 & -0.179134000 \\ 6 & 3.640388000 & -1.373260000 & 1.798067000 \\ 6 & 5.924895000 & -1.808291000 & 0.278207000 \\ 1 & 5.049103000 & -0.457164000 & -1.138825000 \\ 6 & 4.633035000 & -2.236943000 & 2.259487000 \\ 1 & 2.762653000 & -1.225043000 & 2.417578000 \\ 6 & 5.777487000 & -2.458051000 & 1.502746000 \\ 1 & 6.813609000 & -1.972647000 & -0.323572000 \\ 1 & 4.505444000 & -2.733940000 & 3.216303000 \\ 1 & 6.550184000 & -3.130770000 & 1.862208000 \\ 6 & 3.260464000 & 1.502251000 & -0.539977000 \\ 6 & 4.346166000 & 2.121636000 & 0.083934000 \\ 6 & 2.629416000 & 2.161066000 & -1.597815000 \\ 6 & 4.781936000 & 3.378185000 & -0.328141000 \\ 1 & 4.859230000 & 1.623318000 & 0.900823000 \\ 6 & 3.062279000 & 3.417959000 & -2.009276000 \\ 1 & 1.793433000 & 1.681541000 & -2.094249000 \\ 6 & 4.137636000 & 4.033918000 & -1.373552000 \\ 1 & 5.628487000 & 3.842458000 & 0.169024000 \\ 1 & 2.558547000 & 3.916077000 & -2.832716000 \\ 1 & 4.476476000 & 5.014048000 & -1.696007000 \\ 1 & -2.537448000 & -0.657353000 & -1.272844000 \\ 1 & -4.130616000 & -1.877090000 & -1.801984000 \\ 6 & -6.375138000 & 0.756365000 & -2.239489000 \\ 1 & -6.252301000 & 1.592266000 & -2.935701000 \\ 1 & -6.762892000 & 1.164311000 & -1.297512000 \\ 1 & -7.126104000 & 0.073474000 & -2.645407000 \\ 6 & -4.849921000 & 2.840723000 & 1.443574000 \\ 1 & -5.821208000 & 3.257643000 & 1.177774000 \\ 1 & -4.615244000 & 3.063504000 & 2.487513000 \\ 1 & -4.070516000 & 3.265266000 & 0.803246000 \\ 8 & -4.961124000 & 1.439491000 & 1.230066000 \\ 6 & -3.854745000 & 0.730909000 & 1.517461000 \\ 8 & -2.866330000 & 1.234803000 & 2.031810000 \\ 6 & -3.939349000 & -0.694717000 & 1.170186000 \\ 16 & -5.282652000 & -1.413436000 & 0.521324000 \\ 16 & -2.424752000 & -1.522509000 & 1.629198000 \\ 6 & -2.785451000 & -3.240217000 & 1.201196000 \\ 1 & -3.647797000 & -3.613819000 & 1.758228000 \\ 1 & -1.895265000 & -3.811579000 & 1.477436000 \\ 1 & -2.970317000 & -3.360057000 & 0.131122000\end{array}$




\section{$\mathrm{TS}_{1}-\mathrm{DF}$}

$\mathrm{HF}=-2571.8049342$ hartrees

Imaginary Frequencies: $1(-251.54661 / \mathrm{cm})$

Zero-point correction $=0.665684$ (Hartree/Particle)

Temperature: 298.150 Kelvin. Pressure: 1.00000 Atm.

Thermal correction to Gibbs Free Energy $=0.592512$

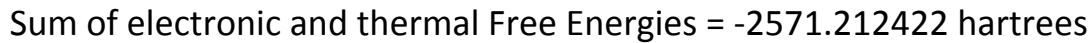

Single Point Energy (wB97xd/cc-pVDZ in chloroform) Single Point Energy (wB97xd/cc-pVTZ in chloroform) Single Point Energy (wB97xd/cc-pVQZ in chloroform) Single Point Energy (wB97xd/tzvp in chloroform) Single Point Energy (M062x/tzvp in chloroform)

Coordinates:

$\begin{array}{lccc}6 & -4.011155000 & 0.379841000 & 2.009220000 \\ 6 & -5.073966000 & -0.535115000 & 1.935974000 \\ 6 & -4.912478000 & -1.846423000 & 1.467231000 \\ 6 & -2.675239000 & 0.094823000 & 1.779287000 \\ 6 & -1.657105000 & 1.063530000 & 1.724884000 \\ 6 & -0.330492000 & 0.695443000 & 1.651996000 \\ 7 & 0.698580000 & 1.540506000 & 1.541316000 \\ 6 & 2.113953000 & 1.153435000 & 1.615348000 \\ 6 & 2.829975000 & 2.497908000 & 1.824070000 \\ 6 & 1.890995000 & 3.565818000 & 1.244096000 \\ 6 & 0.491275000 & 2.982595000 & 1.432306000 \\ 1 & -4.268138000 & 1.420883000 & 2.203655000 \\ 1 & -5.751831000 & -2.532041000 & 1.558733000 \\ 1 & -1.939276000 & 2.112022000 & 1.734413000 \\ 1 & -0.057391000 & -0.353575000 & 1.686339000 \\ 1 & 2.232815000 & 0.499520000 & 2.482913000 \\ 1 & 3.817577000 & 2.513372000 & 1.358190000 \\ 1 & 2.972420000 & 2.659937000 & 2.896977000 \\ 1 & 2.091884000 & 3.720751000 & 0.182661000 \\ 1 & 1.999953000 & 4.526557000 & 1.752091000 \\ 1 & -0.175348000 & 3.193919000 & 0.589182000 \\ 1 & 0.012916000 & 3.352534000 & 2.348850000 \\ 6 & 2.576123000 & 0.308905000 & 0.376969000 \\ 8 & 1.795272000 & -0.867795000 & 0.492744000 \\ 14 & 1.891947000 & -2.446872000 & -0.076737000 \\ 6 & 1.793890000 & -2.486963000 & -1.949953000 \\ 1 & 1.750874000 & -3.522634000 & -2.308340000 \\ 1 & 2.666087000 & -2.010863000 & -2.410617000\end{array}$

$\mathrm{HF}=-2571.936166$ hartrees $\mathrm{HF}=-2572.396602$ hartrees $\mathrm{HF}=-2572.523673$ hartrees $\mathrm{HF}=-2572.334345$ hartrees $\mathrm{HF}=-2572.131352$ hartrees 


\begin{tabular}{|c|c|c|c|}
\hline & 0.902273000 & -1.962099000 & -2.309993000 \\
\hline & 0.387357000 & -3.236634000 & 0.717007000 \\
\hline & -0.510204000 & -2.637138000 & 0.532436000 \\
\hline & 0.527609000 & -3.324850000 & 1.800470000 \\
\hline & 0.212711000 & -4.242818000 & 0.318452000 \\
\hline & 3.417178000 & -3.356540000 & 0.535926000 \\
\hline & 3.281321000 & -4.432095000 & 0.366347000 \\
\hline & 3.552356000 & -3.202696000 & 1.612154000 \\
\hline & 4.338038000 & -3.045761000 & 0.034994000 \\
\hline & 4.061835000 & -0.072785000 & 0.465122000 \\
\hline & 4.763403000 & -0.424187000 & -0.693506000 \\
\hline & 4.714420000 & -0.215068000 & 1.692916000 \\
\hline & 6.072061000 & -0.891495000 & -0.630905000 \\
\hline & 4.281323000 & -0.332 & -1.661087000 \\
\hline & 6.024137000 & -0.682199000 & 1.760997000 \\
\hline & 4.210382000 & 0.032006000 & 2.620894000 \\
\hline & 6.709359000 & -1.02 & 000 \\
\hline & 6.590983000 & -1.155340000 & -1.547545000 \\
\hline & 6.506757000 & -0.782202000 & 2.728489000 \\
\hline & 7.730057000 & 39826000 & 0.65277 \\
\hline & 2.230232000 & 1.075597000 & -0.902118000 \\
\hline & 3.109526000 & 2.006796000 & -1.465562000 \\
\hline & 0.957594000 & 0.943698000 & -1.456385000 \\
\hline & 2.726052000 & 2.775504000 & -2.5605 \\
\hline & 4.102833000 & 2.140649000 & -1.048145000 \\
\hline & 0.567136000 & 1.714794000 & -2.547318000 \\
\hline & 0.255738000 & 0.239328000 & -1.02 \\
\hline & 1.451618000 & 2.633017000 & -3.105057000 \\
\hline & 3.426082000 & 3.489726000 & -2.984507000 \\
\hline & -0.439341000 & 1.598267000 & -2.935952000 \\
\hline & 1.151243000 & 3.236405000 & -3.956836000 \\
\hline & -2.369381000 & -0.942982000 & 1.658856000 \\
\hline & -3.941696000 & -2.329099000 & 1.529955000 \\
\hline & -6.468627000 & 0.005207000 & 2.118069000 \\
\hline & -6.500631000 & 0.763617000 & 2.906272000 \\
\hline & -7.185317000 & -0.783277000 & 2.362951000 \\
\hline & -6.795477000 & 0.481992000 & 1.185018000 \\
\hline$)$ & -4.789195000 & 2.830723000 & -0.779138000 \\
\hline & -5.824027000 & 3.118911000 & -0.593493000 \\
\hline & -4.152982000 & 3.159322000 & 0.049069000 \\
\hline & -4.428009000 & 3.286549000 & -1.704442000 \\
\hline & -4.796216000 & 1.413547000 & -0.871089000 \\
\hline & -3.595493000 & 0.852171000 & -1.123719000 \\
\hline
\end{tabular}




$\begin{array}{llll}8 & -2.604981000 & 1.523008000 & -1.380723000 \\ 6 & -3.596339000 & -0.611632000 & -1.074251000 \\ 16 & -4.936765000 & -1.525716000 & -0.730522000 \\ 16 & -1.979892000 & -1.255828000 & -1.479589000 \\ 6 & -2.298067000 & -3.025841000 & -1.644048000 \\ 1 & -2.624181000 & -3.471544000 & -0.701476000 \\ 1 & -1.345202000 & -3.473560000 & -1.939401000 \\ 1 & -3.048995000 & -3.220824000 & -2.413765000\end{array}$


$\operatorname{lnt}_{\mathrm{F}}$

$\mathrm{HF}=-2571.8144595$ hartrees

Imaginary Frequencies: none found

Zero-point correction $=0.667544$ (Hartree/Particle)

Temperature: 298.150 Kelvin. Pressure: 1.00000 Atm.

Thermal correction to Gibbs Free Energy $=0.593623$

Sum of electronic and thermal Free Energies $=-2571.220836$ hartrees

Single Point Energy (wB97xd/cc-pVDZ in chloroform) $\quad H F=-2571.9442719$ hartrees

\begin{tabular}{lccc}
\multicolumn{4}{l}{ Coordinates: } \\
6 & -4.103634000 & 0.054342000 & 2.127231000 \\
6 & -5.157171000 & -0.768958000 & 1.867713000 \\
6 & -5.012733000 & -1.977613000 & 1.041275000 \\
6 & -2.748237000 & -0.204720000 & 1.773328000 \\
6 & -1.766495000 & 0.772340000 & 1.822947000 \\
6 & -0.418394000 & 0.454572000 & 1.670866000 \\
7 & 0.557390000 & 1.345115000 & 1.607025000 \\
6 & 1.998079000 & 1.029181000 & 1.670174000 \\
6 & 2.638000000 & 2.399477000 & 1.945875000 \\
6 & 1.648858000 & 3.438196000 & 1.399804000 \\
6 & 0.281936000 & 2.785799000 & 1.585955000 \\
1 & -4.307767000 & 1.025243000 & 2.576006000 \\
1 & -5.867556000 & -2.653162000 & 1.122293000 \\
1 & -2.068821000 & 1.805358000 & 1.960041000 \\
1 & -0.105417000 & -0.582338000 & 1.607589000 \\
1 & 2.137265000 & 0.345001000 & 2.509673000 \\
1 & 3.627536000 & 2.486822000 & 1.492990000 \\
1 & 2.760483000 & 2.523805000 & 3.026295000 \\
1 & 1.832730000 & 3.627808000 & 0.341014000 \\
1 & 1.716208000 & 4.389277000 & 1.932069000 \\
1 & -0.418717000 & 3.002445000 & 0.773859000 \\
1 & -0.181729000 & 3.076523000 & 2.536611000 \\
6 & 2.518093000 & 0.268376000 & 0.402450000 \\
8 & 1.797968000 & -0.950909000 & 0.446597000 \\
14 & 2.035721000 & -2.498551000 & -0.177608000 \\
6 & 1.993227000 & -2.448185000 & -2.051991000 \\
1 & 1.967444000 & -3.463170000 & -2.465573000 \\
1 & 2.868947000 & -1.938916000 & -2.467668000 \\
1 & 1.101895000 & -1.913759000 & -2.396998000 \\
6 & 0.590715000 & -3.455640000 & 0.531896000
\end{tabular}




\begin{tabular}{|c|c|c|c|}
\hline & -0.369503000 & -3.009253000 & 0.258170000 \\
\hline & 0.659573000 & -3.493172000 & 1.625562000 \\
\hline & 0.600071000 & -4.488278000 & 0.163191000 \\
\hline & 3.605976000 & -3.317902000 & 0.451479000 \\
\hline & 3.542836000 & -4.394928000 & 0.251337000 \\
\hline & 3.702960000 & -3.186095000 & 1.534801000 \\
\hline & 4.518029000 & -2.937112000 & -0.014878000 \\
\hline & 4.022595000 & -0.033333000 & 0.513342000 \\
\hline & 4.770497000 & -0.290936000 & -0.641279000 \\
\hline & 4.656446000 & -0.189359000 & 1.749277000 \\
\hline & 6.104411000 & -0.677561000 & -0.566585000 \\
\hline & 4.306304000 & -0.188505000 & -1.616289000 \\
\hline & 5.991348000 & -0.576649000 & 1.829571000 \\
\hline & 4.120397000 & -0.014623000 & 2.675695000 \\
\hline & 6.722352000 & -0.822428000 & 0.671967000 \\
\hline & 6.657644000 & -0.868877000 & -1.481075000 \\
\hline & 6.458064000 & -0.688172000 & 2.803553000 \\
\hline & 7.763603000 & -1.123401000 & 0.734925000 \\
\hline & 2.164531000 & 1.081928000 & -0.847228000 \\
\hline & 3.022785000 & 2.072573000 & -1.340993000 \\
\hline & 0.907131000 & 0.938312000 & -1.434576000 \\
\hline & 2.638571000 & 2.886852000 & -2.401302000 \\
\hline & 4.001056000 & 2.219347000 & -0.894284000 \\
\hline & 0.515821000 & 1.762328000 & -2.487154000 \\
\hline & 0.205592000 & 0.196604000 & -1.068219000 \\
\hline & 1.379749000 & 2.735304000 & -2.978148000 \\
\hline & 3.324198000 & 3.644239000 & -2.770207000 \\
\hline & -0.485798000 & 1.651917000 & -2.887956000 \\
\hline & 1.073821000 & 3.377950000 & -3.798672000 \\
\hline & -2.441390000 & -1.228380000 & 1.573346000 \\
\hline & -4.091721000 & -2.540804000 & 1.199812000 \\
\hline & -6.555822000 & -0.365089000 & 2.234414000 \\
\hline & -6.582303000 & 0.598388000 & 2.749820000 \\
\hline & -7.020633000 & -1.119701000 & 2.880244000 \\
\hline & -7.171308000 & -0.290170000 & 1.329455000 \\
\hline & -4.564143000 & 2.904641000 & -0.658529000 \\
\hline & -5.588975000 & 3.237129000 & -0.484662000 \\
\hline & -3.919422000 & 3.250007000 & 0.156855000 \\
\hline & -4.188373000 & 3.323462000 & -1.596573000 \\
\hline & -4.620063000 & 1.490708000 & -0.704487000 \\
\hline & -3.427598000 & 0.873235000 & -0.962081000 \\
\hline & -2.424241000 & 1.543430000 & -1.198811000 \\
\hline & -3.494837000 & -0.562790000 & -0.89683200 \\
\hline
\end{tabular}


$\begin{array}{llll}16 & -4.998894000 & -1.410715000 & -0.767238000\end{array}$

$\begin{array}{llll}16 & -2.028521000 & -1.469013000 & -1.254719000\end{array}$

$6 \begin{array}{llll}6 & -1.970040000 & -1.473385000 & -3.074526000\end{array}$

$\begin{array}{llll}1 & -2.869592000 & -1.940085000 & -3.484838000\end{array}$

$\begin{array}{llll}1 & -1.093592000 & -2.050672000 & -3.384434000\end{array}$

$\begin{array}{llll}1 & -1.878624000 & -0.451473000 & -3.450653000\end{array}$ 


\begin{tabular}{|c|c|c|c|c|}
\hline \multicolumn{5}{|c|}{$\operatorname{lnt}_{\mathrm{DF}}$} \\
\hline \multicolumn{5}{|c|}{$H F=-2571.8112075$ hartrees } \\
\hline \multicolumn{5}{|c|}{ Imaginary Frequencies: none found } \\
\hline \multicolumn{5}{|c|}{ Zero-point correction $=0.666999$ (Hartree/Particle) } \\
\hline \multicolumn{5}{|c|}{ Temperature: 298.150 Kelvin. Pressure: 1.00000 Atm. } \\
\hline \multicolumn{5}{|c|}{ Thermal correction to Gibbs Free Energy= 0.592584} \\
\hline \multicolumn{5}{|c|}{ Sum of electronic and thermal Free Energies $=-2571.218624$ hartrees } \\
\hline \multicolumn{4}{|c|}{ Single Point Energy (wB97xd/cc-pVDZ in chloroform) } & $\mathrm{HF}=-2571.941388$ hartrees \\
\hline \multicolumn{5}{|c|}{ Coordinates: } \\
\hline 6 & -4.103634000 & 0.054342000 & 2.127231000 & \\
\hline 6 & -5.157171000 & -0.768958000 & 1.867713000 & \\
\hline 6 & -5.012733000 & -1.977613000 & 1.041275000 & \\
\hline 6 & -2.748237000 & -0.204720000 & 1.773328000 & \\
\hline 6 & -1.766495000 & 0.772340000 & 1.822947000 & \\
\hline 6 & -0.418394000 & 0.454572000 & 1.670866000 & \\
\hline 7 & 0.557390000 & 1.345115000 & 1.607025000 & \\
\hline 6 & 1.998079000 & 1.029181000 & 1.670174000 & \\
\hline 6 & 2.638000000 & 2.399477000 & 1.945875000 & \\
\hline 6 & 1.648858000 & 3.438196000 & 1.399804000 & \\
\hline 6 & 0.281936000 & 2.785799000 & 1.585955000 & \\
\hline 1 & -4.307767000 & 1.025243000 & 2.576006000 & \\
\hline 1 & -5.867556000 & -2.653162000 & 1.122293000 & \\
\hline 1 & -2.068821000 & 1.805358000 & 1.960041000 & \\
\hline 1 & -0.105417000 & -0.582338000 & 1.607589000 & \\
\hline 1 & 2.137265000 & 0.345001000 & 2.509673000 & \\
\hline 1 & 3.627536000 & 2.486822000 & 1.492990000 & \\
\hline 1 & 2.760483000 & 2.523805000 & 3.026295000 & \\
\hline 1 & 1.832730000 & 3.627808000 & 0.341014000 & \\
\hline 1 & 1.716208000 & 4.389277000 & 1.932069000 & \\
\hline 1 & -0.418717000 & 3.002445000 & 0.773859000 & \\
\hline 1 & -0.181729000 & 3.076523000 & 2.536611000 & \\
\hline 6 & 2.518093000 & 0.268376000 & 0.402450000 & \\
\hline 8 & 1.797968000 & -0.950909000 & 0.446597000 & \\
\hline 14 & 2.035721000 & -2.498551000 & -0.177608000 & \\
\hline 6 & 1.993227000 & -2.448185000 & -2.051991000 & \\
\hline 1 & 1.967444000 & -3.463170000 & -2.465573000 & \\
\hline 1 & 2.868947000 & -1.938916000 & -2.467668000 & \\
\hline 1 & 1.101895000 & -1.913759000 & -2.396998000 & \\
\hline 6 & 0.590715000 & -3.455640000 & 0.531896000 & \\
\hline 1 & -0.369503000 & -3.009253000 & 0.258170000 & \\
\hline
\end{tabular}




\begin{tabular}{llll}
1 & 0.659573000 & -3.493172000 & 1.625562000 \\
1 & 0.600071000 & -4.488278000 & 0.163191000 \\
6 & 3.605976000 & -3.317902000 & 0.451479000 \\
1 & 3.542836000 & -4.394928000 & 0.251337000 \\
1 & 3.702960000 & -3.186095000 & 1.534801000 \\
1 & 4.518029000 & -2.937112000 & -0.014878000 \\
6 & 4.022595000 & -0.033333000 & 0.513342000 \\
6 & 4.770497000 & -0.290936000 & -0.641279000 \\
6 & 4.656446000 & -0.189359000 & 1.749277000 \\
6 & 6.104411000 & -0.677561000 & -0.566585000 \\
1 & 4.306304000 & -0.188505000 & -1.616289000 \\
6 & 5.991348000 & -0.576649000 & 1.829571000 \\
1 & 4.120397000 & -0.014623000 & 2.675695000 \\
6 & 6.722352000 & -0.822428000 & 0.671967000 \\
1 & 6.657644000 & -0.868877000 & -1.481075000 \\
1 & 6.458064000 & -0.688172000 & 2.803553000 \\
1 & 7.763603000 & -1.123401000 & 0.734925000 \\
6 & 2.164531000 & 1.081928000 & -0.847228000 \\
6 & 3.022785000 & 2.072573000 & -1.340993000 \\
6 & 0.907131000 & 0.938312000 & -1.434576000 \\
6 & 2.638571000 & 2.886852000 & -2.401302000 \\
1 & 4.001056000 & 2.219347000 & -0.894284000 \\
6 & 0.515821000 & 1.762328000 & -2.487154000 \\
1 & 0.205592000 & 0.196604000 & -1.068219000 \\
6 & 1.379749000 & 2.735304000 & -2.978148000 \\
1 & 3.324198000 & 3.644239000 & -2.770207000 \\
1 & -0.485798000 & 1.651917000 & -2.887956000 \\
1 & 1.073821000 & 3.377950000 & -3.798672000 \\
1 & -2.441390000 & -1.228380000 & 1.573346000 \\
1 & -4.091721000 & -2.540804000 & 1.199812000 \\
6 & -6.555822000 & -0.365089000 & 2.234414000 \\
1 & -6.582303000 & 0.598388000 & 2.749820000 \\
1 & -7.020633000 & -1.119701000 & 2.880244000 \\
1 & -7.171308000 & -0.290170000 & 1.329455000 \\
6 & -4.564143000 & 2.904641000 & -0.658529000 \\
1 & -5.588975000 & 3.237129000 & -0.484662000 \\
1 & -3.919422000 & 3.250007000 & 0.156855000 \\
1 & -4.188373000 & 3.323462000 & -1.596573000 \\
8 & -4.620063000 & 1.490708000 & -0.704487000 \\
6 & -3.427598000 & 0.873235000 & -0.962081000 \\
\hline 6 & -2.424241000 & 1.543430000 & -1.198811000 \\
\hline & -4.998894000 & -1.410715000 & -0.767238000
\end{tabular}


$\begin{array}{llll}16 & -2.028521000 & -1.469013000 & -1.254719000\end{array}$

$6 \begin{array}{llll}6 & -1.970040000 & -1.473385000 & -3.074526000\end{array}$

$\begin{array}{llll}1 & -2.869592000 & -1.940085000 & -3.484838000\end{array}$

$\begin{array}{llll}1 & -1.093592000 & -2.050672000 & -3.384434000\end{array}$

$\begin{array}{llll}1 & -1.878624000 & -0.451473000 & -3.450653000\end{array}$ 


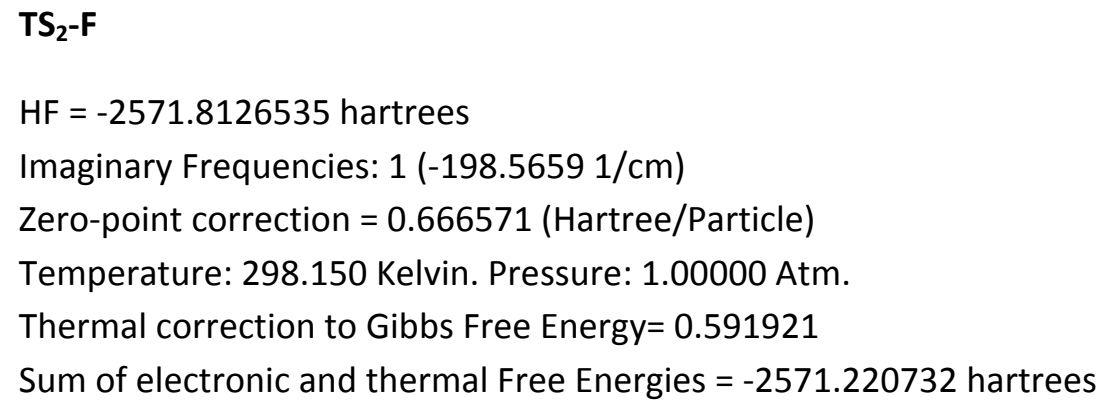

$\begin{array}{lccc}6 & -3.847451000 & 0.818342000 & -1.854438000 \\ 6 & -4.994754000 & 0.164836000 & -2.152483000 \\ 6 & -5.217606000 & -1.216741000 & -1.671218000 \\ 6 & -2.731979000 & 0.226445000 & -1.164354000 \\ 6 & -1.677545000 & 1.018981000 & -0.674576000 \\ 6 & -0.549563000 & 0.427854000 & -0.149102000 \\ 7 & 0.404772000 & 1.043788000 & 0.542406000 \\ 6 & 1.587002000 & 0.359626000 & 1.059585000 \\ 6 & 1.977894000 & 1.218516000 & 2.274405000 \\ 6 & 1.420929000 & 2.623988000 & 1.991308000 \\ 6 & 0.222258000 & 2.396373000 & 1.065365000 \\ 1 & -3.781418000 & 1.885251000 & -2.061581000 \\ 1 & -6.127502000 & -1.663880000 & -2.078198000 \\ 1 & -1.832500000 & 2.089367000 & -0.578152000 \\ 1 & -0.411997000 & -0.638546000 & -0.276756000 \\ 1 & 1.283228000 & -0.644854000 & 1.367879000 \\ 1 & 3.056255000 & 1.230236000 & 2.446516000 \\ 1 & 1.505027000 & 0.795825000 & 3.165821000 \\ 1 & 2.169909000 & 3.242789000 & 1.493736000 \\ 1 & 1.122996000 & 3.131857000 & 2.911113000 \\ 1 & 0.191359000 & 3.124486000 & 0.247941000 \\ 1 & -0.738467000 & 2.419877000 & 1.594359000 \\ 6 & 2.682421000 & 0.181794000 & -0.048052000 \\ 8 & 2.076641000 & -0.522582000 & -1.119669000 \\ 14 & 1.824832000 & -2.164149000 & -1.407581000 \\ 6 & 3.383419000 & -3.046729000 & -1.966220000 \\ 1 & 3.111478000 & -4.016567000 & -2.400893000 \\ 1 & 4.072760000 & -3.227244000 & -1.135691000 \\ 1 & 3.918070000 & -2.473292000 & -2.730959000 \\ 6 & 0.571167000 & -2.138184000 & -2.801168000 \\ 1 & -0.328943000 & -1.578000000 & -2.525225000\end{array}$




$\begin{array}{llll}1 & 0.266869000 & -3.157111000 & -3.066512000 \\ 1 & 0.993610000 & -1.668181000 & -3.695944000 \\ 6 & 1.128563000 & -3.069179000 & 0.087361000 \\ 1 & 0.939584000 & -4.112292000 & -0.195714000 \\ 1 & 0.180862000 & -2.654494000 & 0.449304000 \\ 1 & 1.840932000 & -3.082163000 & 0.919290000 \\ 6 & 3.901460000 & -0.603276000 & 0.451086000 \\ 6 & 5.024327000 & -0.671108000 & -0.382601000 \\ 6 & 3.922755000 & -1.322013000 & 1.647163000 \\ 6 & 6.133111000 & -1.429962000 & -0.033564000 \\ 1 & 5.020297000 & -0.126269000 & -1.321800000 \\ 6 & 5.032678000 & -2.089820000 & 1.999536000 \\ 1 & 3.078116000 & -1.298689000 & 2.327753000 \\ 6 & 6.140704000 & -2.146621000 & 1.162141000 \\ 1 & 6.990378000 & -1.467403000 & -0.698945000 \\ 1 & 5.025760000 & -2.639980000 & 2.935575000 \\ 1 & 7.005372000 & -2.742483000 & 1.438306000 \\ 6 & 3.081198000 & 1.560405000 & -0.580748000 \\ 6 & 4.126119000 & 2.291364000 & -0.009508000 \\ 6 & 2.332394000 & 2.147609000 & -1.602773000 \\ 6 & 4.406781000 & 3.585547000 & -0.437851000 \\ 1 & 4.729610000 & 1.850498000 & 0.777929000 \\ 6 & 2.610534000 & 3.442527000 & -2.031167000 \\ 1 & 1.526686000 & 1.582245000 & -2.057396000 \\ 6 & 3.645989000 & 4.168317000 & -1.447632000 \\ 1 & 5.224012000 & 4.136134000 & 0.018524000 \\ 1 & 2.016412000 & 3.883996000 & -2.826066000 \\ 1 & 3.862865000 & 5.178280000 & -1.782632000 \\ 1 & -2.526632000 & -0.826728000 & -1.337973000 \\ 1 & -4.383494000 & -1.900837000 & -1.843830000 \\ 6 & -6.158314000 & 0.861978000 & -2.795430000 \\ 1 & -5.932587000 & 1.907474000 & -3.021911000 \\ 1 & -7.029000000 & 0.834046000 & -2.128036000 \\ 1 & -6.449006000 & 0.358443000 & -3.725210000 \\ 6 & -4.444569000 & 2.723906000 & 1.923109000 \\ 1 & -5.326170000 & 3.324815000 & 1.697332000 \\ 1 & -4.335076000 & 2.606182000 & 3.004569000 \\ 1 & -3.548193000 & 3.212687000 & 1.529617000 \\ 8 & -4.661222000 & 1.472125000 & 1.288049000 \\ 6 & -3.688244000 & 0.548521000 & 1.486189000 \\ 6 & -2.716510000 & 0.795158000 & 2.191461000 \\ 16 & -3.881539000 & -0.677732000 & 0.730033000 \\ & -5.484540000 & -1.127744000 & 0.181992000\end{array}$


$\begin{array}{llll}16 & -2.723144000 & -1.987419000 & 1.050504000\end{array}$

$\begin{array}{llll}6 & -3.077549000 & -2.427569000 & 2.777629000\end{array}$

$\begin{array}{llll}1 & -2.922083000 & -1.554637000 & 3.414502000\end{array}$

$\begin{array}{llll}1 & -2.381572000 & -3.219312000 & 3.067911000\end{array}$

$\begin{array}{llll}1 & -4.102735000 & -2.792937000 & 2.879916000\end{array}$ 


\section{$\mathrm{TS}_{\mathbf{2}}-\mathrm{DF}$}

$\mathrm{HF}=-2571.8105764$ hartrees

Imaginary Frequencies: $1(-116.24461 / \mathrm{cm})$

Zero-point correction $=0.666066$ (Hartree/Particle)

Temperature: 298.150 Kelvin. Pressure: 1.00000 Atm.

Thermal correction to Gibbs Free Energy $=0.593016$

Sum of electronic and thermal Free Energies $=-2571.217561$ hartrees

Single Point Energy (wB97xd/cc-pVDZ in chloroform) $\quad H F=-2571.940814$ hartrees

Coordinates:

$\begin{array}{lccc}6 & -4.130573000 & -0.117185000 & 2.113255000 \\ 6 & -5.188130000 & -0.930150000 & 1.865717000 \\ 6 & -5.069225000 & -2.047824000 & 0.909753000 \\ 6 & -2.804730000 & -0.326490000 & 1.609426000 \\ 6 & -1.812204000 & 0.657146000 & 1.732459000 \\ 6 & -0.471058000 & 0.355818000 & 1.593537000 \\ 7 & 0.513867000 & 1.253194000 & 1.606818000 \\ 6 & 1.947954000 & 0.934476000 & 1.705457000 \\ 6 & 2.579025000 & 2.292290000 & 2.058277000 \\ 6 & 1.600880000 & 3.350903000 & 1.531616000 \\ 6 & 0.233539000 & 2.687762000 & 1.666798000 \\ 1 & -4.300360000 & 0.805487000 & 2.665798000 \\ 1 & -5.941140000 & -2.706005000 & 0.911269000 \\ 1 & -2.125769000 & 1.680474000 & 1.913361000 \\ 1 & -0.152469000 & -0.674043000 & 1.471952000 \\ 1 & 2.066516000 & 0.214477000 & 2.518672000 \\ 1 & 3.579871000 & 2.401133000 & 1.635326000 \\ 1 & 2.672963000 & 2.370181000 & 3.146032000 \\ 1 & 1.805266000 & 3.575466000 & 0.482947000 \\ 1 & 1.657085000 & 4.283509000 & 2.097308000 \\ 1 & -0.458666000 & 2.953766000 & 0.861418000 \\ 1 & -0.242568000 & 2.927150000 & 2.626996000 \\ 6 & 2.518696000 & 0.231933000 & 0.425022000 \\ 8 & 1.813430000 & -0.998396000 & 0.394920000 \\ 14 & 2.061889000 & -2.490985000 & -0.342445000 \\ 6 & 2.013376000 & -2.311401000 & -2.209626000 \\ 1 & 2.086457000 & -3.293231000 & -2.691996000 \\ 1 & 2.836962000 & -1.692453000 & -2.580942000 \\ 1 & 1.076699000 & -1.840474000 & -2.526312000 \\ 6 & 0.629377000 & -3.516082000 & 0.295072000 \\ 1 & -0.336587000 & -3.054342000 & 0.071701000 \\ & & \end{array}$




\begin{tabular}{llll}
1 & 0.708818000 & -3.647645000 & 1.380531000 \\
1 & 0.641252000 & -4.512353000 & -0.162607000 \\
6 & 3.643513000 & -3.340467000 & 0.214825000 \\
1 & 3.585032000 & -4.403025000 & -0.052674000 \\
1 & 3.753913000 & -3.276519000 & 1.302999000 \\
1 & 4.546465000 & -2.922437000 & -0.237051000 \\
6 & 4.022319000 & -0.059910000 & 0.569820000 \\
6 & 4.810980000 & -0.259069000 & -0.569088000 \\
6 & 4.619467000 & -0.259398000 & 1.817937000 \\
6 & 6.148517000 & -0.626865000 & -0.467873000 \\
1 & 4.375681000 & -0.126222000 & -1.553871000 \\
6 & 5.957526000 & -0.628712000 & 1.925082000 \\
1 & 4.051477000 & -0.130691000 & 2.732683000 \\
6 & 6.729651000 & -0.812994000 & 0.782737000 \\
1 & 6.733112000 & -0.771812000 & -1.371351000 \\
1 & 6.395472000 & -0.772874000 & 2.908176000 \\
1 & 7.773728000 & -1.098599000 & 0.867085000 \\
6 & 2.191995000 & 1.092947000 & -0.799186000 \\
6 & 3.057065000 & 2.102066000 & -1.239844000 \\
6 & 0.944286000 & 0.972777000 & -1.412204000 \\
6 & 2.690451000 & 2.955238000 & -2.275992000 \\
1 & 4.027016000 & 2.232338000 & -0.770391000 \\
6 & 0.570507000 & 1.834568000 & -2.439705000 \\
1 & 0.237153000 & 0.220859000 & -1.079692000 \\
6 & 1.442372000 & 2.824865000 & -2.880115000 \\
1 & 3.381683000 & 3.725919000 & -2.604699000 \\
1 & -0.423150000 & 1.739574000 & -2.863262000 \\
1 & 1.150165000 & 3.497402000 & -3.681475000 \\
1 & -2.479144000 & -1.346251000 & 1.419410000 \\
1 & -4.166525000 & -2.652479000 & 1.017327000 \\
6 & -6.562445000 & -0.606256000 & 2.375320000 \\
1 & -6.565507000 & 0.296320000 & 2.992367000 \\
1 & -6.963502000 & -1.435982000 & 2.969590000 \\
1 & -7.249564000 & -0.450888000 & 1.534060000 \\
6 & -4.484096000 & 3.021889000 & -0.596662000 \\
1 & -5.489850000 & 3.368649000 & -0.355711000 \\
1 & -3.773100000 & 3.389476000 & 0.149707000 \\
1 & -4.182482000 & 3.396809000 & -1.578713000 \\
8 & -4.551956000 & 1.604643000 & -0.583174000 \\
6 & -3.392366000 & 0.970159000 & -0.901509000 \\
\hline 6 & -2.389974000 & 1.608861000 & -1.199522000 \\
& -5.468440000 & -0.474273000 & -0.783941000 \\
\hline
\end{tabular}


$\begin{array}{llll}16 & -2.041744000 & -1.389819000 & -1.291120000\end{array}$

$6 \quad-2.072070000 \quad-1.222782000 \quad-3.101618000$

$\begin{array}{llll}1 & -2.983772000 & -1.662593000 & -3.514701000\end{array}$

$\begin{array}{llll}1 & -1.203583000 & -1.753038000 & -3.502897000\end{array}$

$\begin{array}{llll}1 & -2.008063000 & -0.167432000 & -3.376074000\end{array}$ 
$\mathrm{HF}=-2571.8457698$ hartrees

Imaginary Frequencies: none found

Zero-point correction $=0.669584$ (Hartree/Particle)

Temperature: 298.150 Kelvin. Pressure: 1.00000 Atm.

Thermal correction to Gibbs Free Energy $=0.597868$

Sum of electronic and thermal Free Energies $=-2571.247902$ hartrees

Single Point Energy (wB97xd/cc-pVDZ in chloroform) $\quad H F=-2571.976438$ hartrees

Coordinates:

$\begin{array}{lccc}6 & -3.680010000 & 0.754464000 & -1.618256000 \\ 6 & -4.751596000 & 0.211939000 & -2.195223000 \\ 6 & -5.189402000 & -1.149524000 & -1.735335000 \\ 6 & -2.868883000 & 0.045506000 & -0.558559000 \\ 6 & -1.762627000 & 0.925834000 & -0.042495000 \\ 6 & -0.545968000 & 0.437911000 & 0.263334000 \\ 7 & 0.507620000 & 1.133563000 & 0.775331000 \\ 6 & 1.752694000 & 0.504372000 & 1.187750000 \\ 6 & 2.299238000 & 1.477324000 & 2.249993000 \\ 6 & 1.665663000 & 2.838503000 & 1.925609000 \\ 6 & 0.329553000 & 2.486944000 & 1.268772000 \\ 1 & -3.344275000 & 1.747669000 & -1.906366000 \\ 1 & -6.177875000 & -1.414231000 & -2.121159000 \\ 1 & -1.998219000 & 1.977375000 & 0.098770000 \\ 1 & -0.347937000 & -0.614527000 & 0.104430000 \\ 1 & 1.523078000 & -0.468131000 & 1.635695000 \\ 1 & 3.390759000 & 1.521689000 & 2.260008000 \\ 1 & 1.974830000 & 1.134908000 & 3.237958000 \\ 1 & 2.293450000 & 3.398395000 & 1.228927000 \\ 1 & 1.529485000 & 3.449624000 & 2.821064000 \\ 1 & 0.080518000 & 3.171827000 & 0.448208000 \\ 1 & -0.506777000 & 2.508084000 & 1.985062000 \\ 6 & 2.712254000 & 0.221054000 & -0.021636000 \\ 8 & 2.003158000 & -0.597746000 & -0.942831000 \\ 14 & 1.715697000 & -2.250921000 & -1.014176000 \\ 6 & 3.208604000 & -3.233571000 & -1.586169000 \\ 1 & 2.889090000 & -4.246312000 & -1.862290000 \\ 1 & 3.967172000 & -3.319071000 & -0.801822000 \\ 1 & 3.678893000 & -2.776755000 & -2.463293000 \\ 6 & 0.353782000 & -2.360528000 & -2.301269000 \\ 1 & -0.458817000 & -1.659731000 & -2.079272000\end{array}$




\begin{tabular}{llll}
1 & -0.067140000 & -3.371455000 & -2.350356000 \\
1 & 0.740220000 & -2.108521000 & -3.294929000 \\
6 & 1.143767000 & -2.973979000 & 0.627956000 \\
1 & 0.895288000 & -4.031910000 & 0.475145000 \\
1 & 0.258225000 & -2.485506000 & 1.048850000 \\
1 & 1.941875000 & -2.932464000 & 1.377338000 \\
6 & 3.996214000 & -0.506704000 & 0.394735000 \\
6 & 5.009918000 & -0.657749000 & -0.559459000 \\
6 & 4.169911000 & -1.109527000 & 1.641624000 \\
6 & 6.160067000 & -1.382556000 & -0.278036000 \\
1 & 4.885961000 & -0.203990000 & -1.538135000 \\
6 & 5.320791000 & -1.844000000 & 1.926886000 \\
1 & 3.413020000 & -1.019004000 & 2.413368000 \\
6 & 6.319288000 & -1.982962000 & 0.970004000 \\
1 & 6.930980000 & -1.484826000 & -1.035932000 \\
1 & 5.432887000 & -2.304539000 & 2.903804000 \\
1 & 7.216865000 & -2.551647000 & 1.193708000 \\
6 & 3.006838000 & 1.541247000 & -0.734211000 \\
6 & 4.114351000 & 2.326330000 & -0.404483000 \\
6 & 2.093694000 & 2.031622000 & -1.671126000 \\
6 & 4.301509000 & 3.576283000 & -0.989198000 \\
1 & 4.841256000 & 1.964839000 & 0.316394000 \\
6 & 2.278140000 & 3.281039000 & -2.255124000 \\
1 & 1.233080000 & 1.427671000 & -1.934600000 \\
6 & 3.381342000 & 4.060434000 & -1.914403000 \\
1 & 5.170374000 & 4.169882000 & -0.719996000 \\
1 & 1.556264000 & 3.646001000 & -2.980197000 \\
1 & 3.525891000 & 5.034770000 & -2.371898000 \\
1 & -2.427591000 & -0.844759000 & -1.025704000 \\
1 & -4.488433000 & -1.925862000 & -2.062239000 \\
6 & -5.572483000 & 0.893675000 & -3.251072000 \\
1 & -5.153926000 & 1.869924000 & -3.511720000 \\
1 & -6.602976000 & 1.041227000 & -2.903980000 \\
1 & -5.625146000 & 0.287262000 & -4.163862000 \\
6 & -4.831256000 & 2.794577000 & 1.857126000 \\
1 & -5.240685000 & 3.557316000 & 1.196729000 \\
1 & -5.552812000 & 2.532575000 & 2.633646000 \\
1 & -3.906705000 & 3.145665000 & 2.321548000 \\
6 & -4.568254000 & 1.667338000 & 1.018399000 \\
\hline 6 & -4.043252000 & 0.605255000 & 1.625345000 \\
& -3.819176000 & 0.551944000 & 2.812824000 \\
\hline & -5.355083000 & -1.244025000 & 0.087749000 \\
\hline
\end{tabular}




$\begin{array}{llll}16 & -2.815407000 & -1.776333000 & 1.627636000 \\ 6 & -2.574237000 & -3.082729000 & 0.399082000 \\ 1 & -3.525398000 & -3.365811000 & -0.060637000 \\ 1 & -2.178898000 & -3.941887000 & 0.946528000 \\ 1 & -1.850541000 & -2.808149000 & -0.373745000\end{array}$


$\mathbf{P}_{\mathrm{DF}}$

$\mathrm{HF}=-2571.8437234$ hartrees

Imaginary Frequencies: none found

Zero-point correction $=0.668329$ (Hartree/Particle)

Temperature: 298.150 Kelvin. Pressure: 1.00000 Atm.

Thermal correction to Gibbs Free Energy $=0.595615$

Sum of electronic and thermal Free Energies $=-2571.248108$ hartrees

Single Point Energy (wB97xd/cc-pVDZ in chloroform) $\quad H F=-2571.974803$ hartrees

Coordinates:

$\begin{array}{lccc}6 & -4.094276000 & -0.203597000 & -1.913861000 \\ 6 & -5.295910000 & 0.367063000 & -1.990399000 \\ 6 & -5.597572000 & 1.517029000 & -1.072468000 \\ 6 & -2.993637000 & 0.272951000 & -0.988859000 \\ 6 & -1.826617000 & -0.674741000 & -1.046371000 \\ 6 & -0.559972000 & -0.328280000 & -1.348848000 \\ 7 & 0.467100000 & -1.217311000 & -1.502011000 \\ 6 & 1.859810000 & -0.859506000 & -1.699873000 \\ 6 & 2.476170000 & -2.169012000 & -2.223496000 \\ 6 & 1.579405000 & -3.306835000 & -1.703633000 \\ 6 & 0.238447000 & -2.642591000 & -1.361042000 \\ 1 & -3.851719000 & -1.053036000 & -2.547972000 \\ 1 & -6.661191000 & 1.771977000 & -1.070866000 \\ 1 & -2.073019000 & -1.719647000 & -0.881979000 \\ 1 & -0.280929000 & 0.711566000 & -1.497695000 \\ 1 & 1.907781000 & -0.070091000 & -2.456930000 \\ 1 & 3.517606000 & -2.291014000 & -1.917760000 \\ 1 & 2.462401000 & -2.144212000 & -3.317324000 \\ 1 & 2.010911000 & -3.766633000 & -0.813220000 \\ 1 & 1.458126000 & -4.092204000 & -2.453926000 \\ 1 & -0.089000000 & -2.885719000 & -0.339367000 \\ 1 & -0.565360000 & -2.948854000 & -2.045308000 \\ 6 & 2.538171000 & -0.240188000 & -0.420371000 \\ 8 & 1.939621000 & 1.044664000 & -0.323104000 \\ 14 & 2.406770000 & 2.446059000 & 0.482032000 \\ 6 & 2.720243000 & 2.151246000 & 2.310405000 \\ 1 & 2.884010000 & 3.114245000 & 2.809743000 \\ 1 & 3.603698000 & 1.530924000 & 2.489205000 \\ 1 & 1.862544000 & 1.664341000 & 2.785669000 \\ 6 & 0.900645000 & 3.534817000 & 0.262242000 \\ 1 & 0.026217000 & 3.027548000 & 0.680145000\end{array}$




\begin{tabular}{lccc}
1 & 0.703148000 & 3.731800000 & -0.797417000 \\
1 & 1.020821000 & 4.497344000 & 0.772163000 \\
6 & 3.896696000 & 3.275958000 & -0.307112000 \\
1 & 4.009523000 & 4.282181000 & 0.116218000 \\
1 & 3.759924000 & 3.381951000 & -1.388826000 \\
1 & 4.829615000 & 2.730543000 & -0.136139000 \\
6 & 4.051683000 & -0.056508000 & -0.607975000 \\
6 & 4.929327000 & -0.081348000 & 0.480111000 \\
6 & 4.576327000 & 0.279675000 & -1.861537000 \\
6 & 6.281838000 & 0.210720000 & 0.323657000 \\
1 & 4.555558000 & -0.331120000 & 1.467095000 \\
6 & 5.927241000 & 0.565003000 & -2.024822000 \\
1 & 3.929304000 & 0.330548000 & -2.730612000 \\
6 & 6.788391000 & 0.533434000 & -0.930965000 \\
1 & 6.938672000 & 0.185068000 & 1.188145000 \\
1 & 6.306597000 & 0.818531000 & -3.010278000 \\
1 & 7.843226000 & 0.757750000 & -1.057066000 \\
6 & 2.159003000 & -1.098370000 & 0.788654000 \\
6 & 2.872092000 & -2.253977000 & 1.120739000 \\
6 & 0.992016000 & -0.811613000 & 1.500519000 \\
6 & 2.436533000 & -3.094405000 & 2.141743000 \\
1 & 3.776126000 & -2.509356000 & 0.575154000 \\
6 & 0.547788000 & -1.653129000 & 2.515847000 \\
1 & 0.407577000 & 0.063564000 & 1.238966000 \\
6 & 1.270118000 & -2.798326000 & 2.841865000 \\
1 & 3.009074000 & -3.985235000 & 2.384121000 \\
1 & -0.377491000 & -1.416783000 & 3.031633000 \\
1 & 0.925628000 & -3.457701000 & 3.633305000 \\
1 & -2.667386000 & 1.261409000 & -1.337857000 \\
1 & -5.041988000 & 2.417485000 & -1.357752000 \\
6 & -6.387830000 & -0.097214000 & -2.910190000 \\
1 & -6.057497000 & -0.933779000 & -3.532584000 \\
1 & -6.718449000 & 0.713429000 & -3.571451000 \\
1 & -7.265516000 & -0.419907000 & -2.335816000 \\
6 & -3.969762000 & -3.084324000 & 1.279580000 \\
1 & -4.552622000 & -3.748799000 & 0.644020000 \\
1 & -2.926450000 & -3.407004000 & 1.321671000 \\
1 & -4.384506000 & -3.058793000 & 2.289397000 \\
\hline & -4.063559000 & -1.794281000 & 0.669092000 \\
\hline & -3.387241000 & -0.822421000 & 1.277260000 \\
\hline & -5.207385000 & 1.110817000 & 0.668502000 \\
\hline
\end{tabular}




$\begin{array}{llll}16 & -2.327677000 & 1.633389000 & 1.413950000 \\ 6 & -2.670955000 & 3.201339000 & 0.577238000 \\ 1 & -2.306763000 & 3.216754000 & -0.453743000 \\ 1 & -2.136234000 & 3.969392000 & 1.140940000 \\ 1 & -3.740394000 & 3.426630000 & 0.605003000\end{array}$




\section{6. ${ }^{1} \mathrm{H}$ AND ${ }^{13} \mathrm{C}$ NMR SPECTRA}

Compound $2 a-1$ H NMR

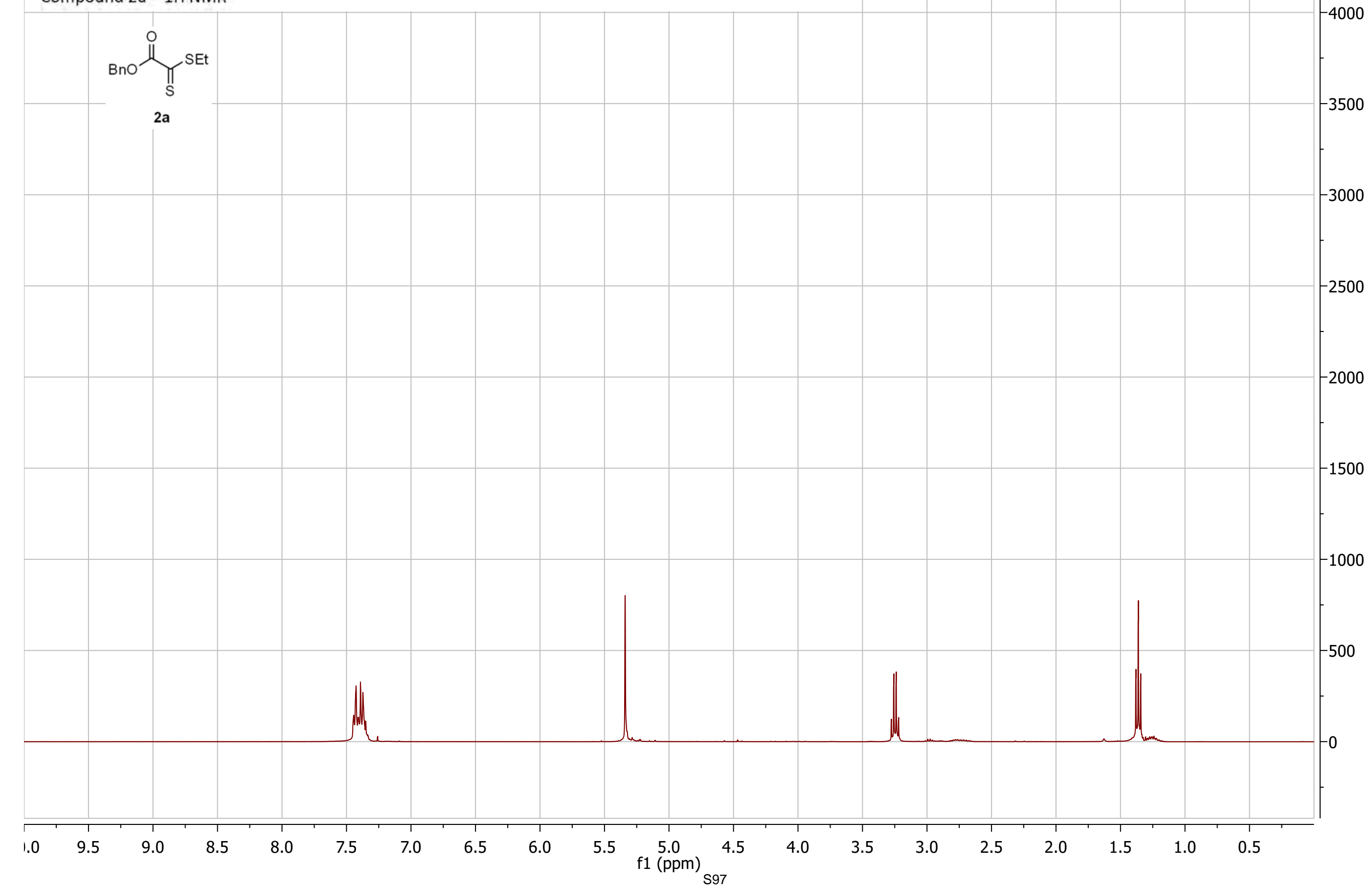




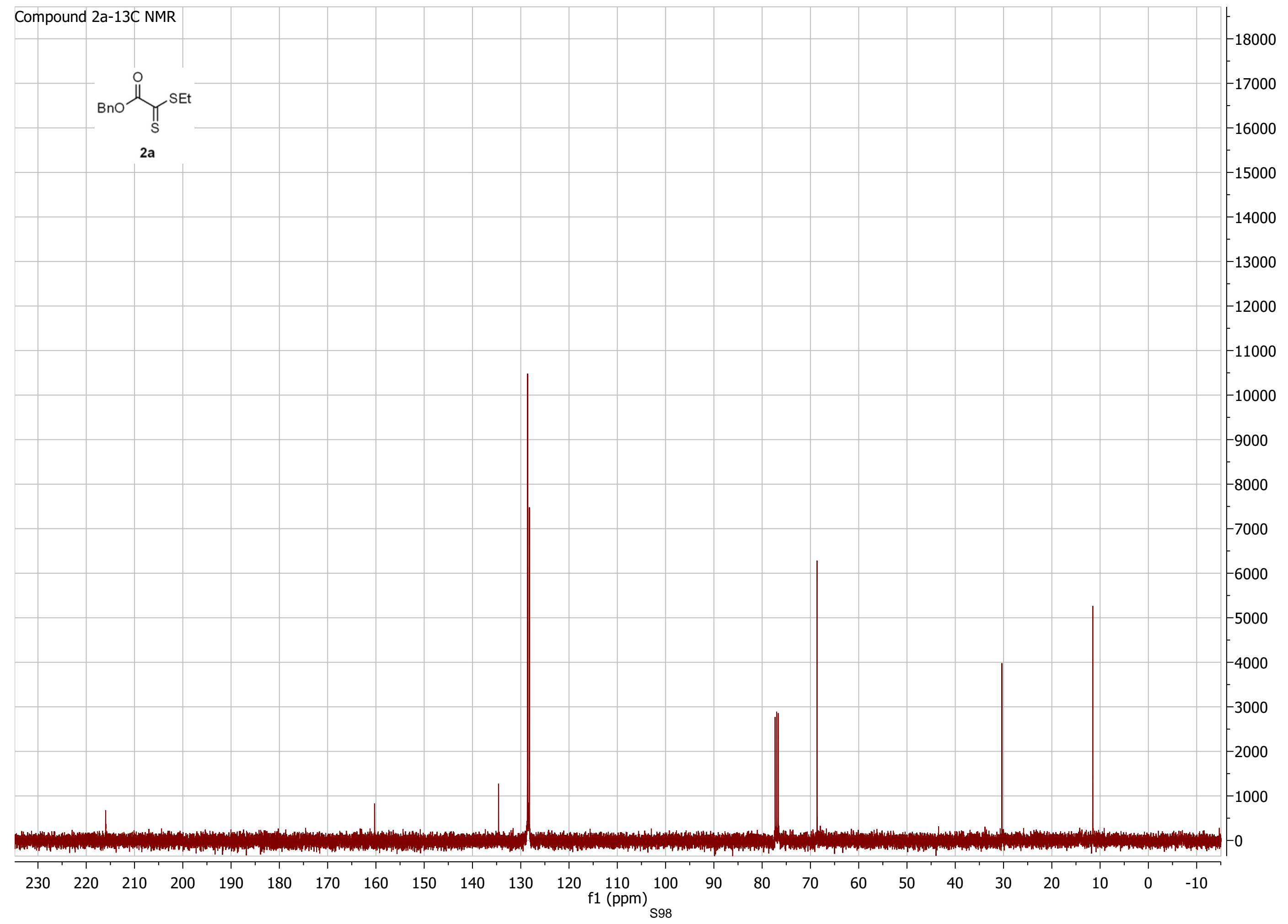




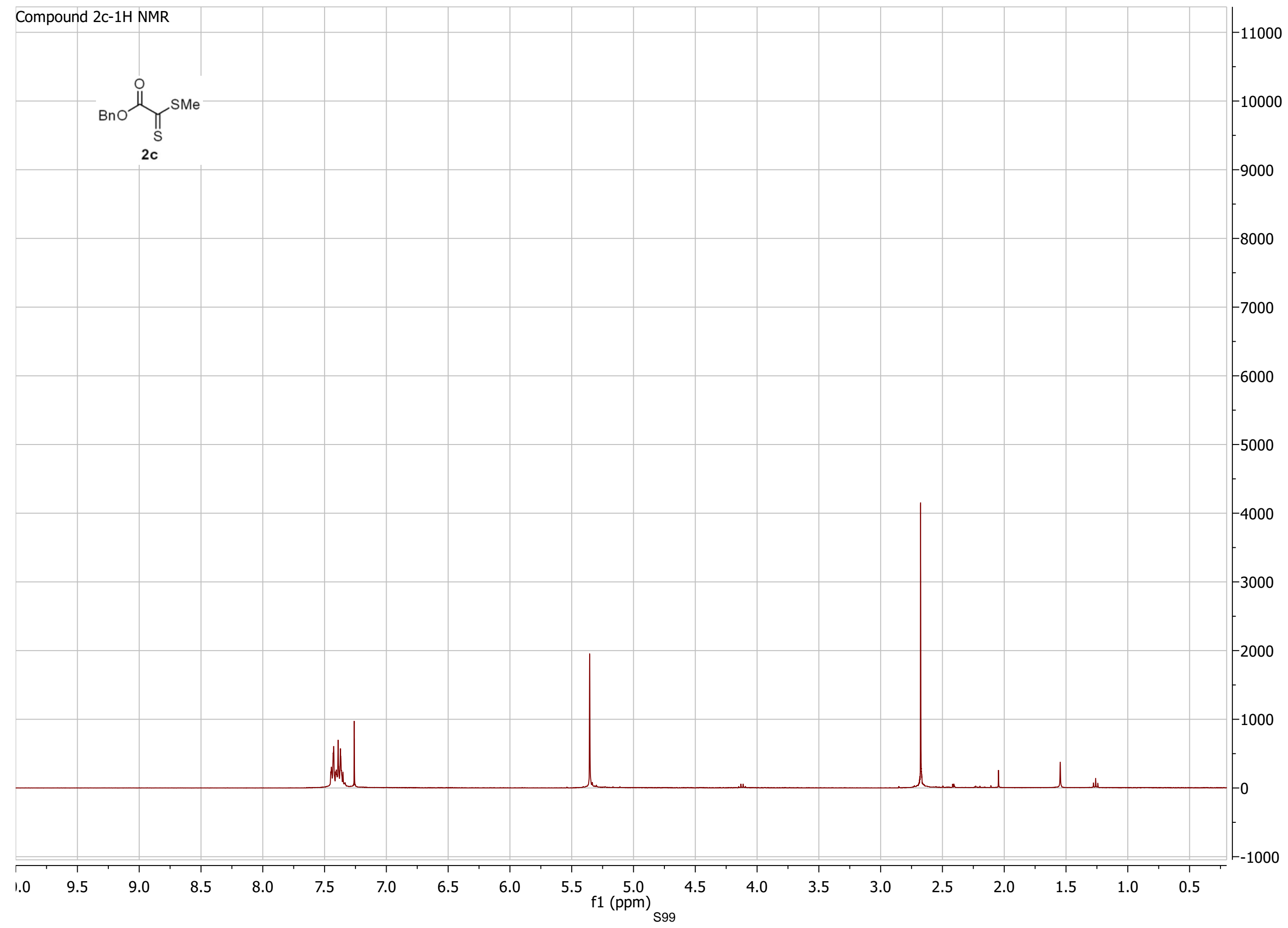




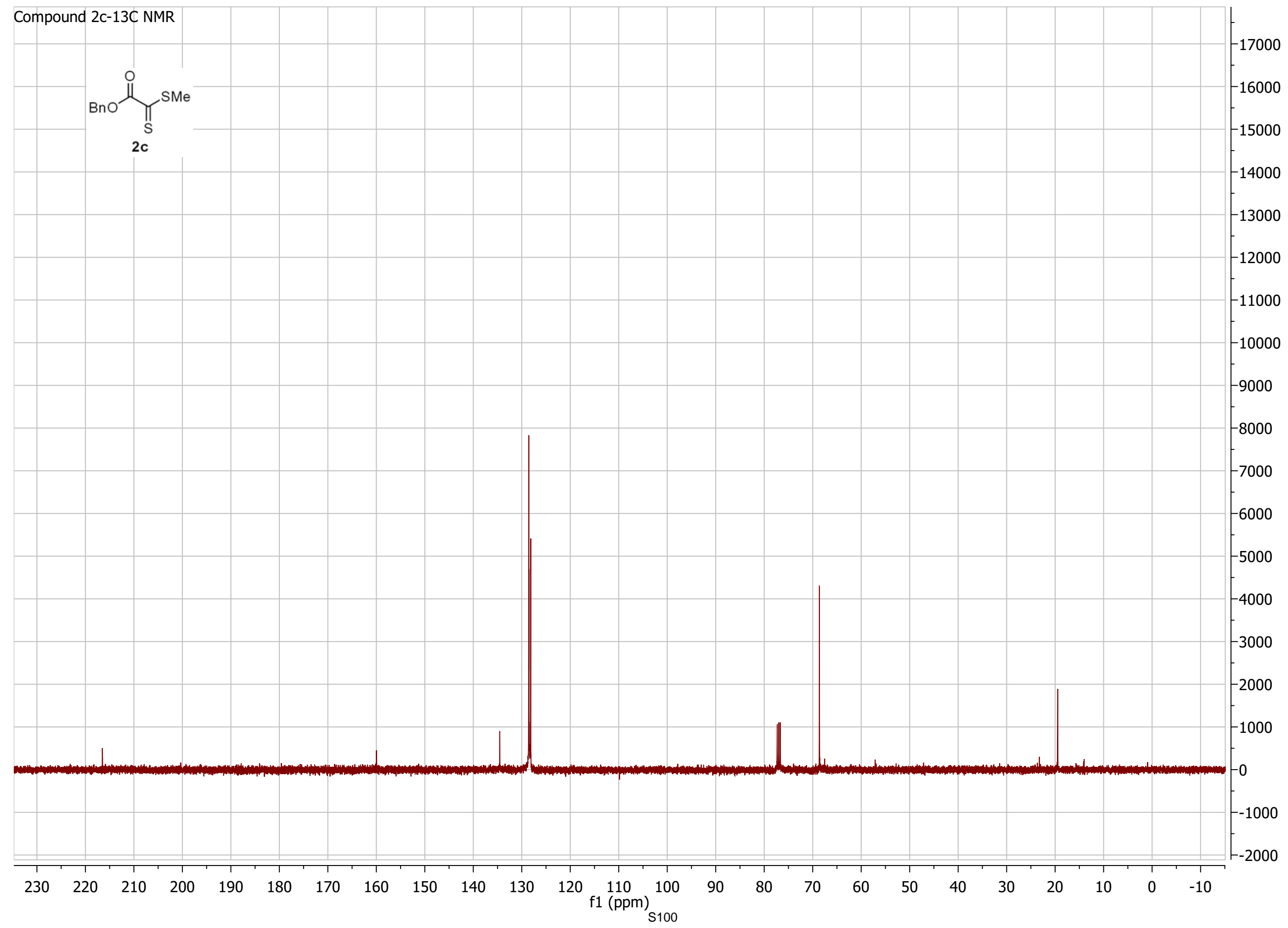




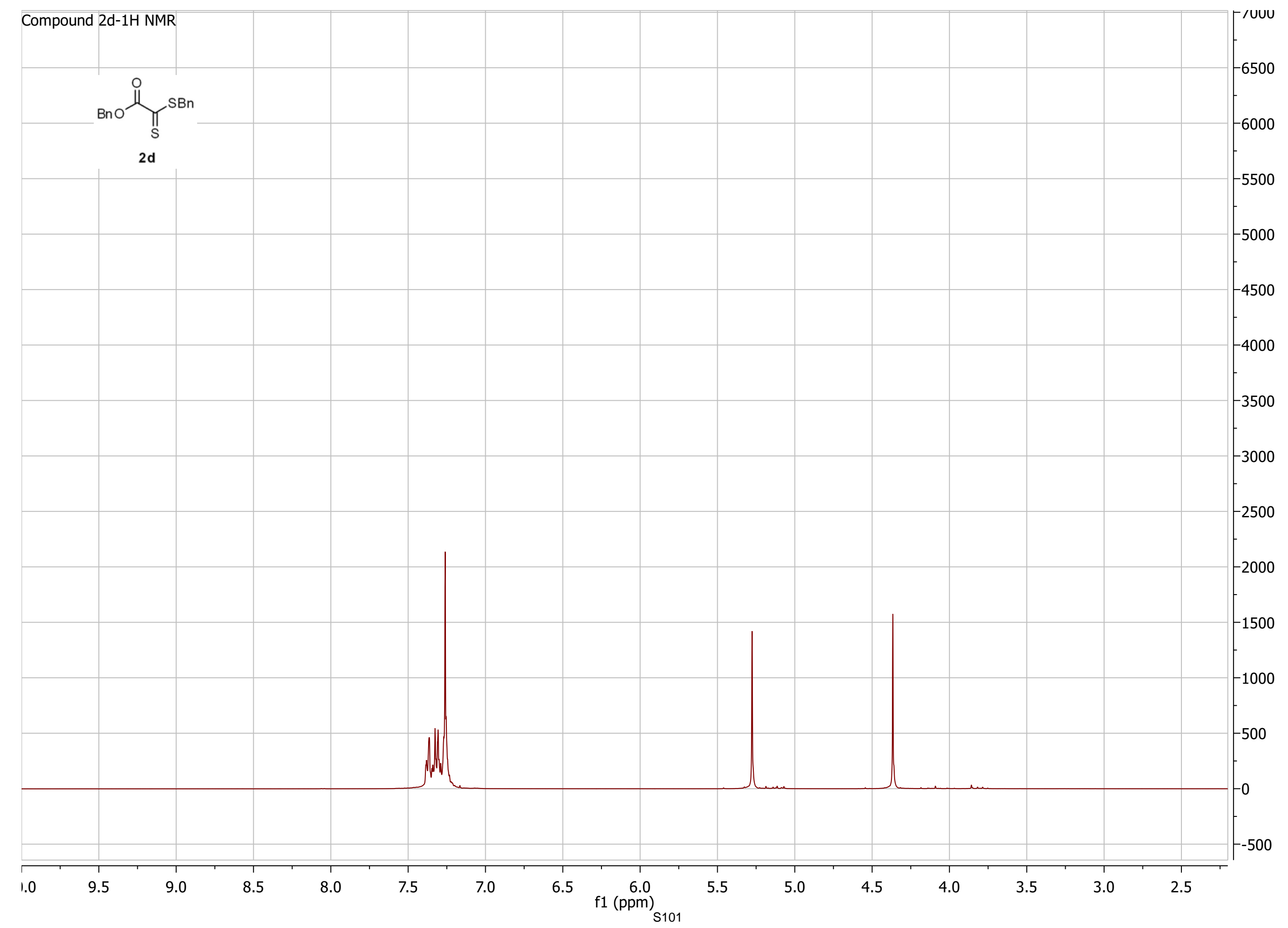




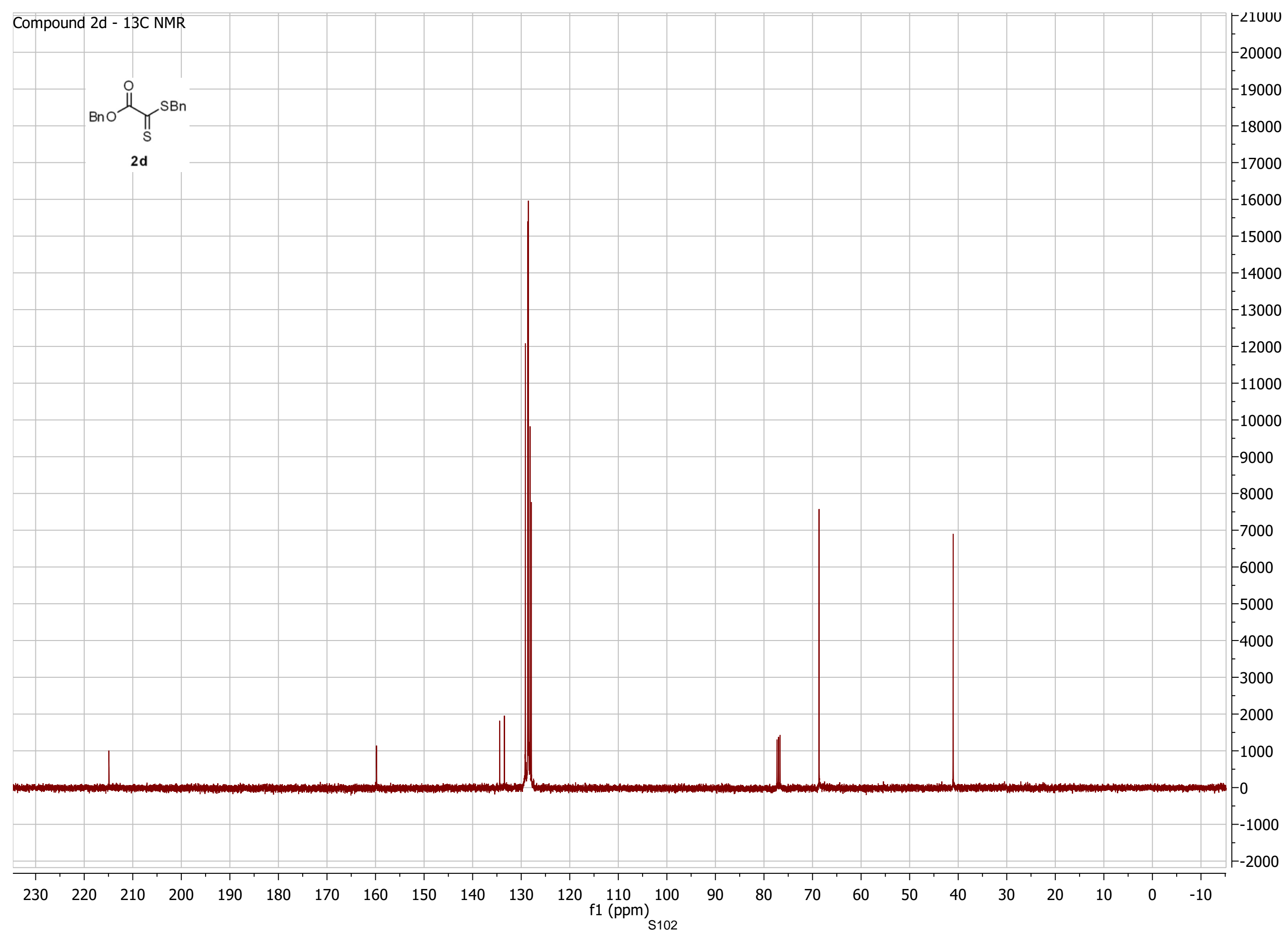




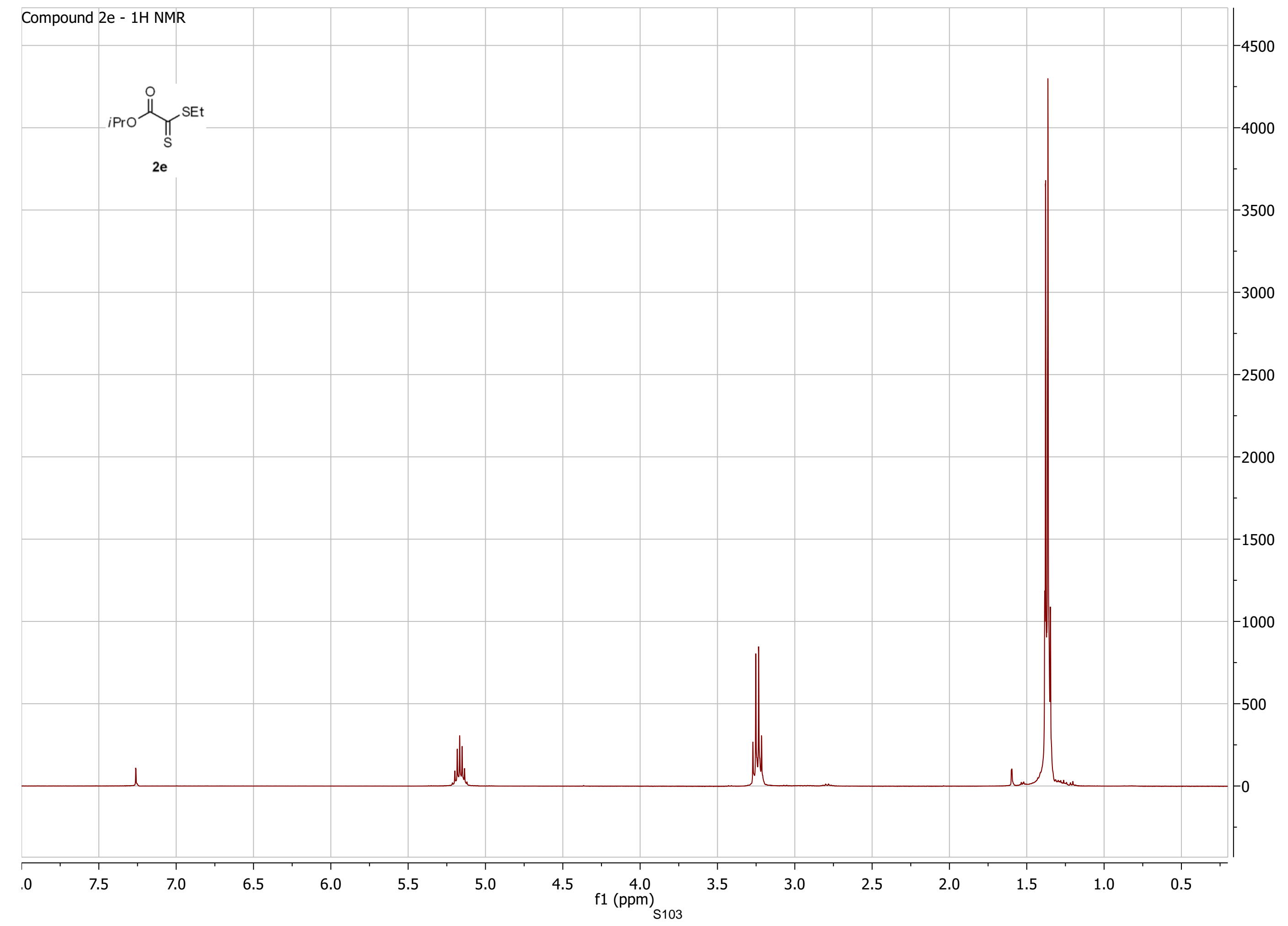




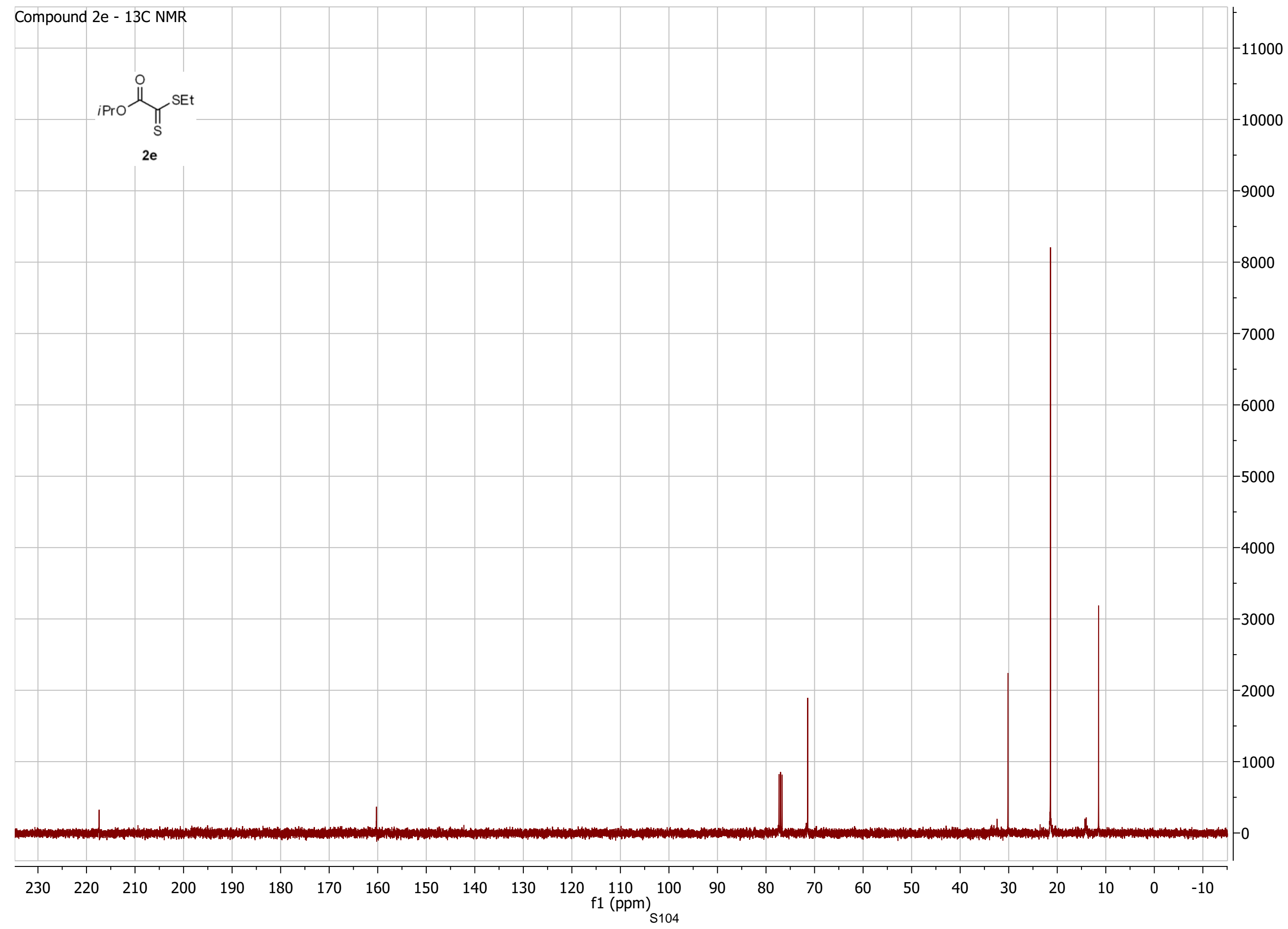




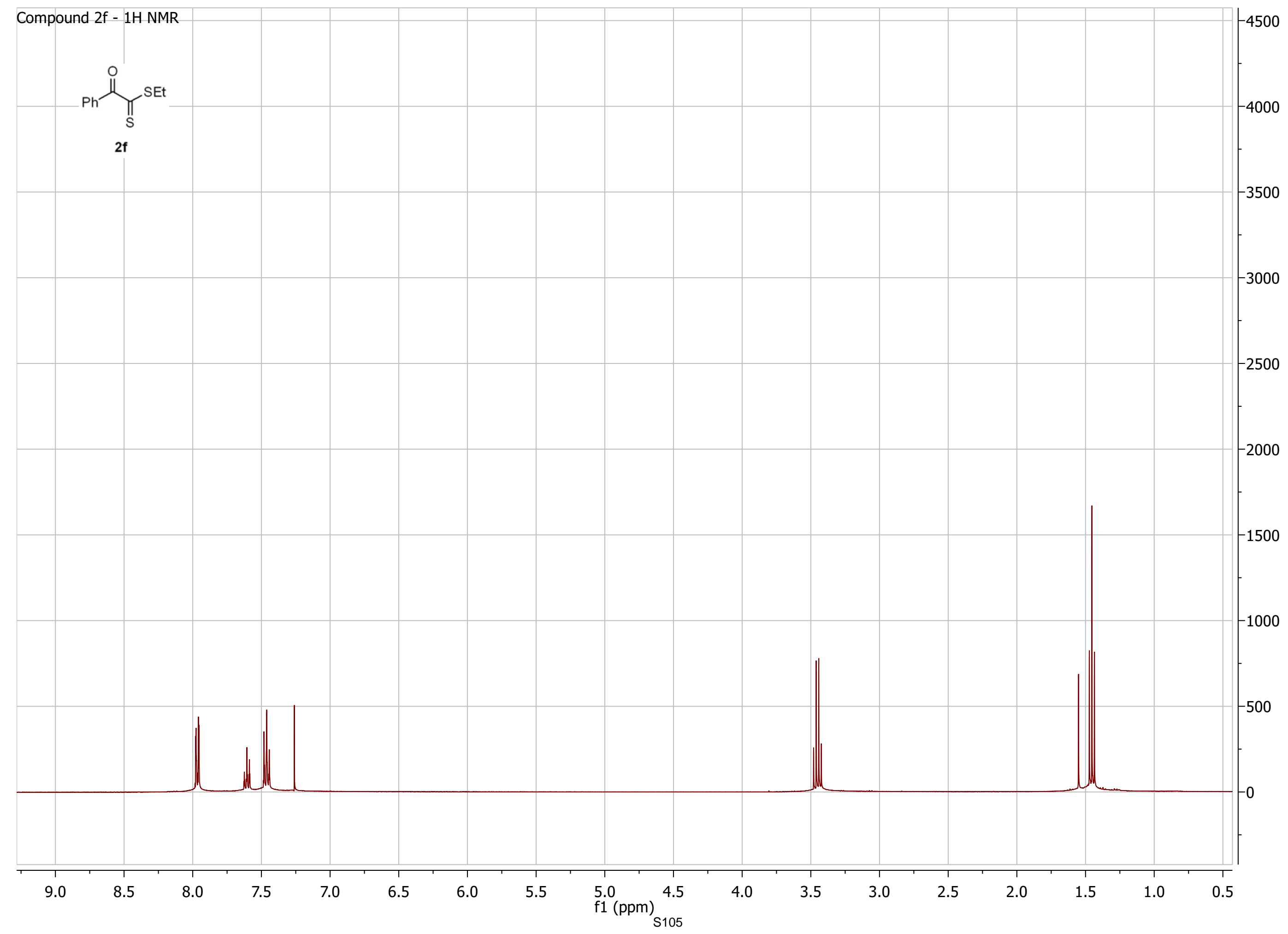



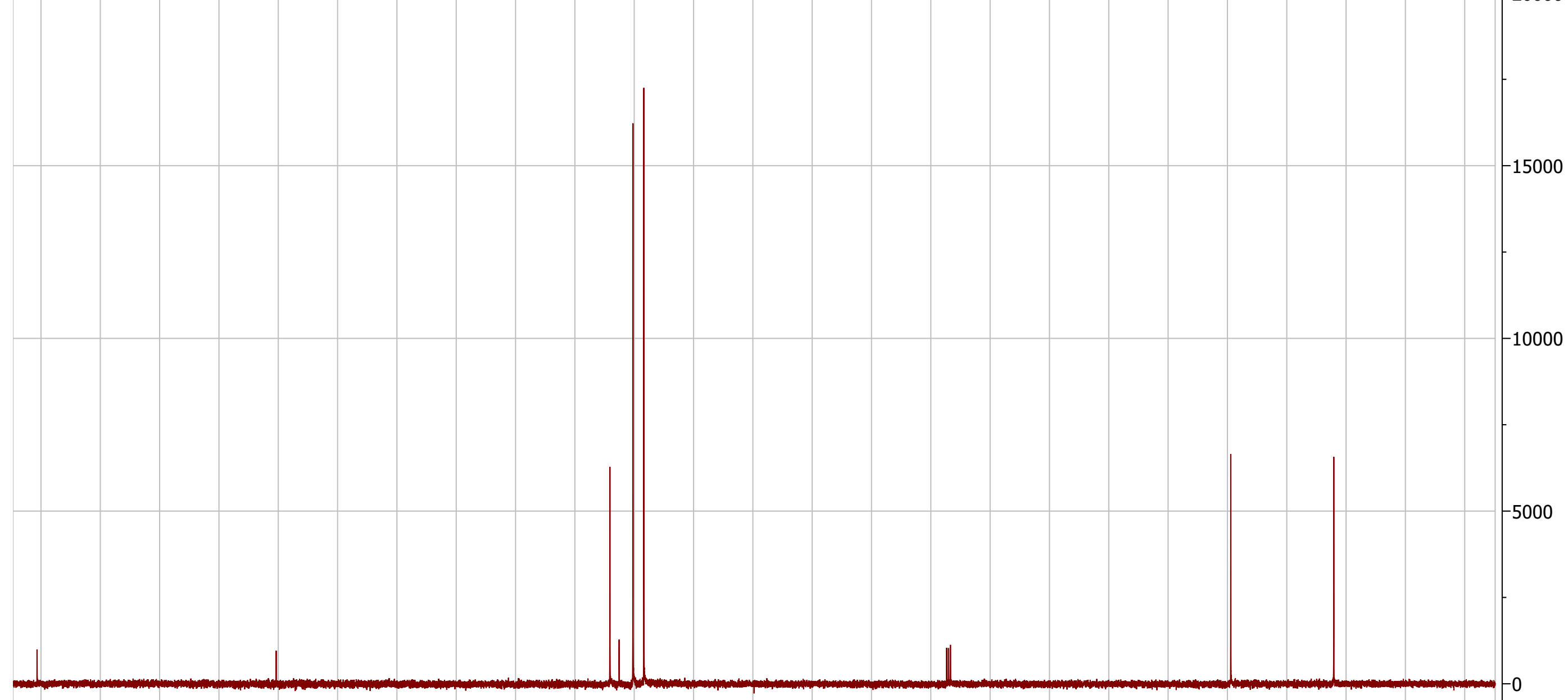

\begin{tabular}{|c|c|c|c|c|c|c|c|c|c|c|c|c|c|c|c|c|c|c|c|c|c|c|c|c|c|c|}
\hline 230 & 220 & 210 & 20 & 19 & 18 & 1 & & 160 & 150 & 140 & 130 & 120 & $\begin{array}{c}110 \\
\text { f1 }(\mathrm{ppm})\end{array}$ & $\left\{\begin{array}{l}100 \\
S 106\end{array}\right.$ & 90 & $\varepsilon$ & 70 & 60 & 5 & & 40 & 30 & 20 & 10 & 0 & -10 \\
\hline
\end{tabular}




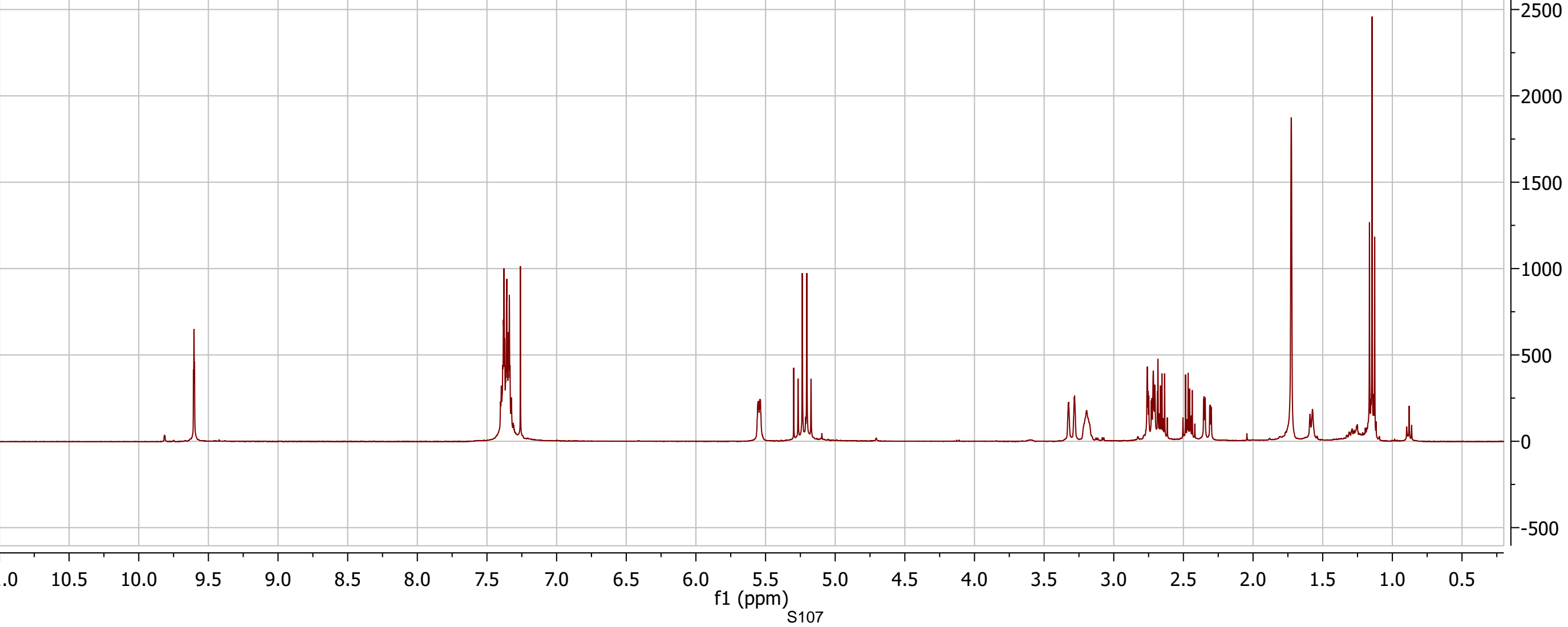




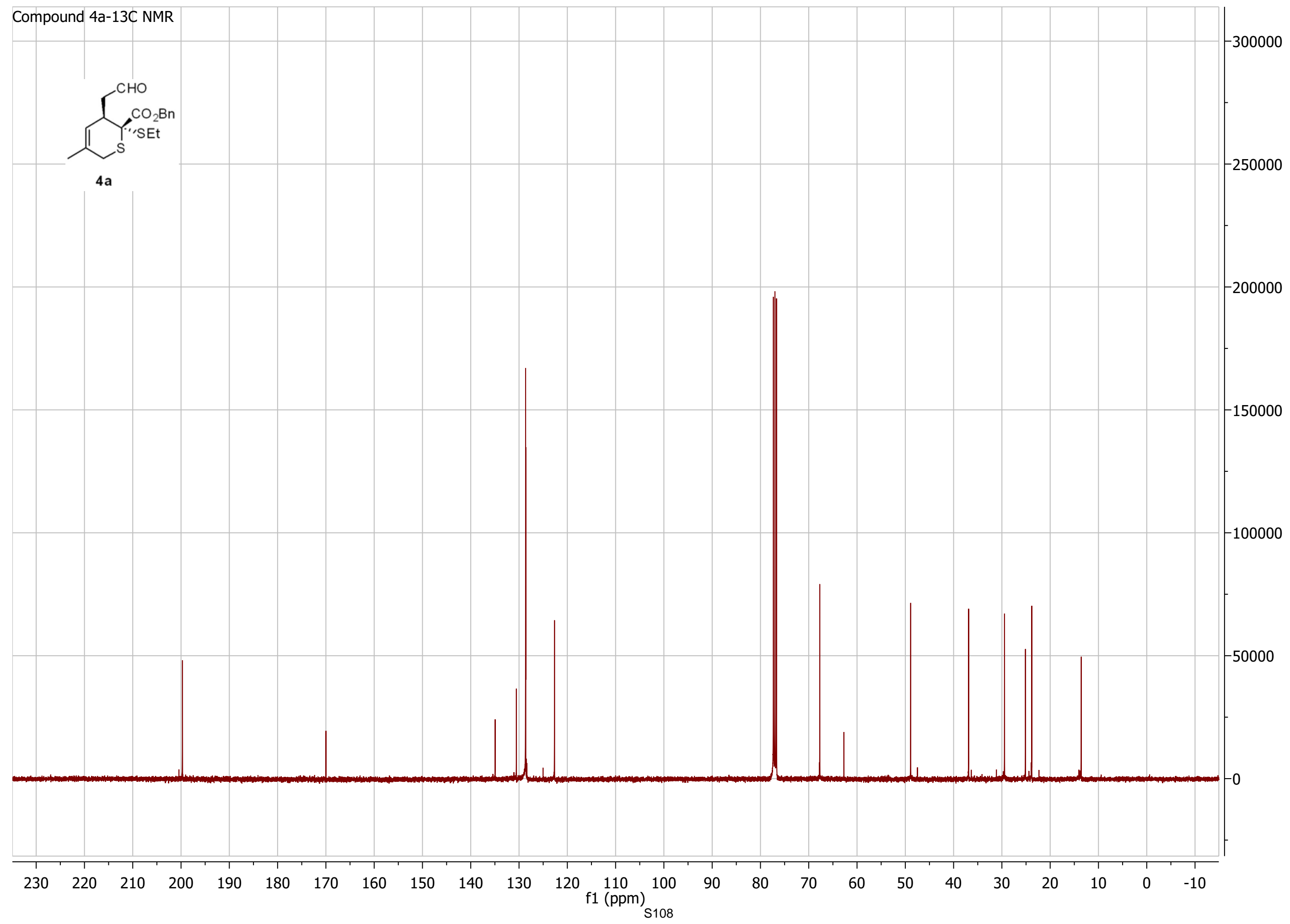




\section{Compound 4b-1H NMR}

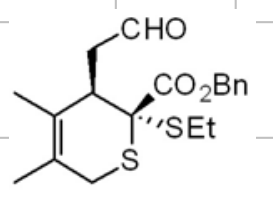

$4 b$ 


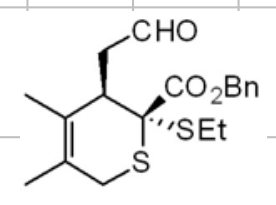

$-24000$

$4 \mathrm{~b}$ 


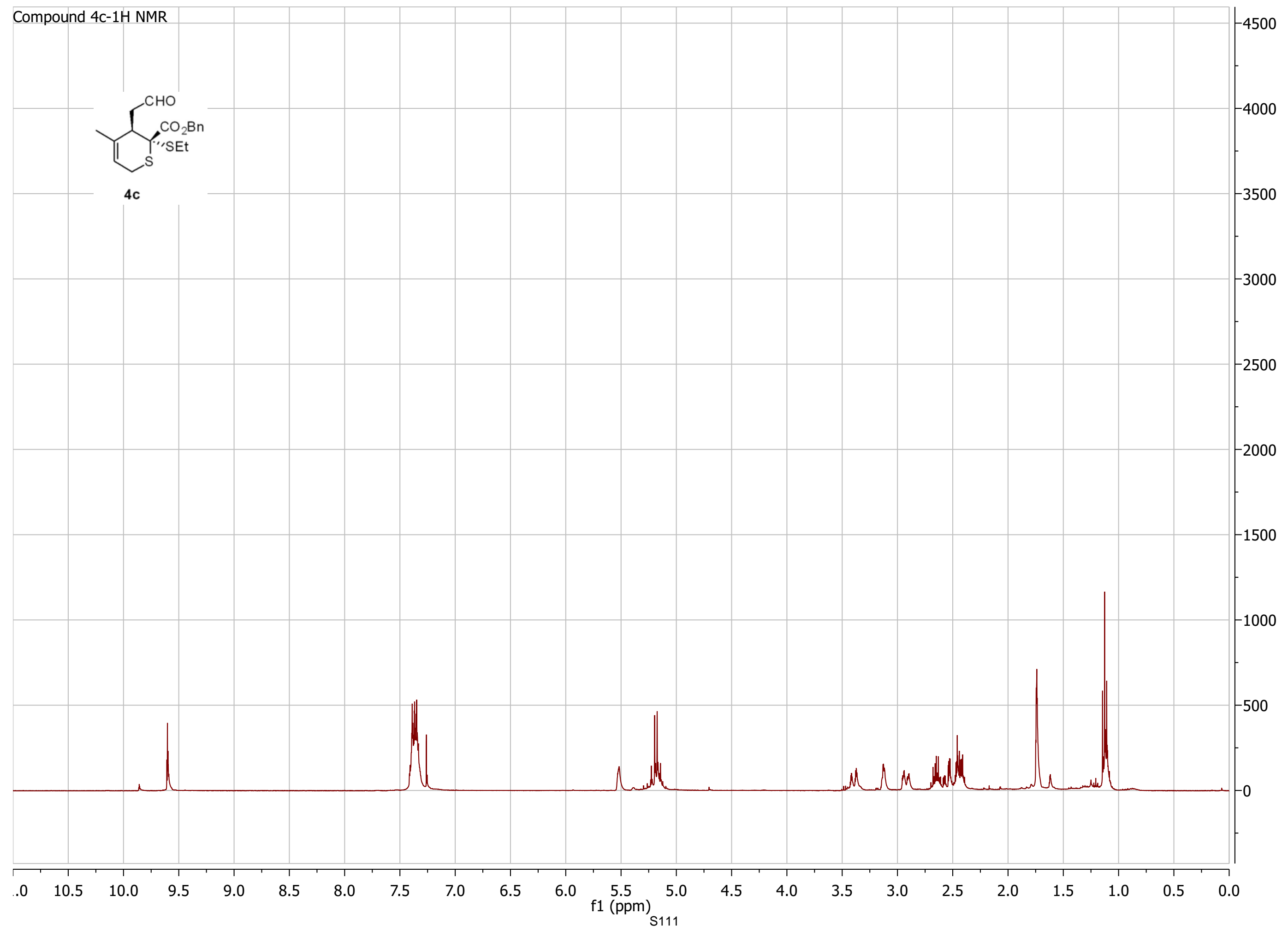




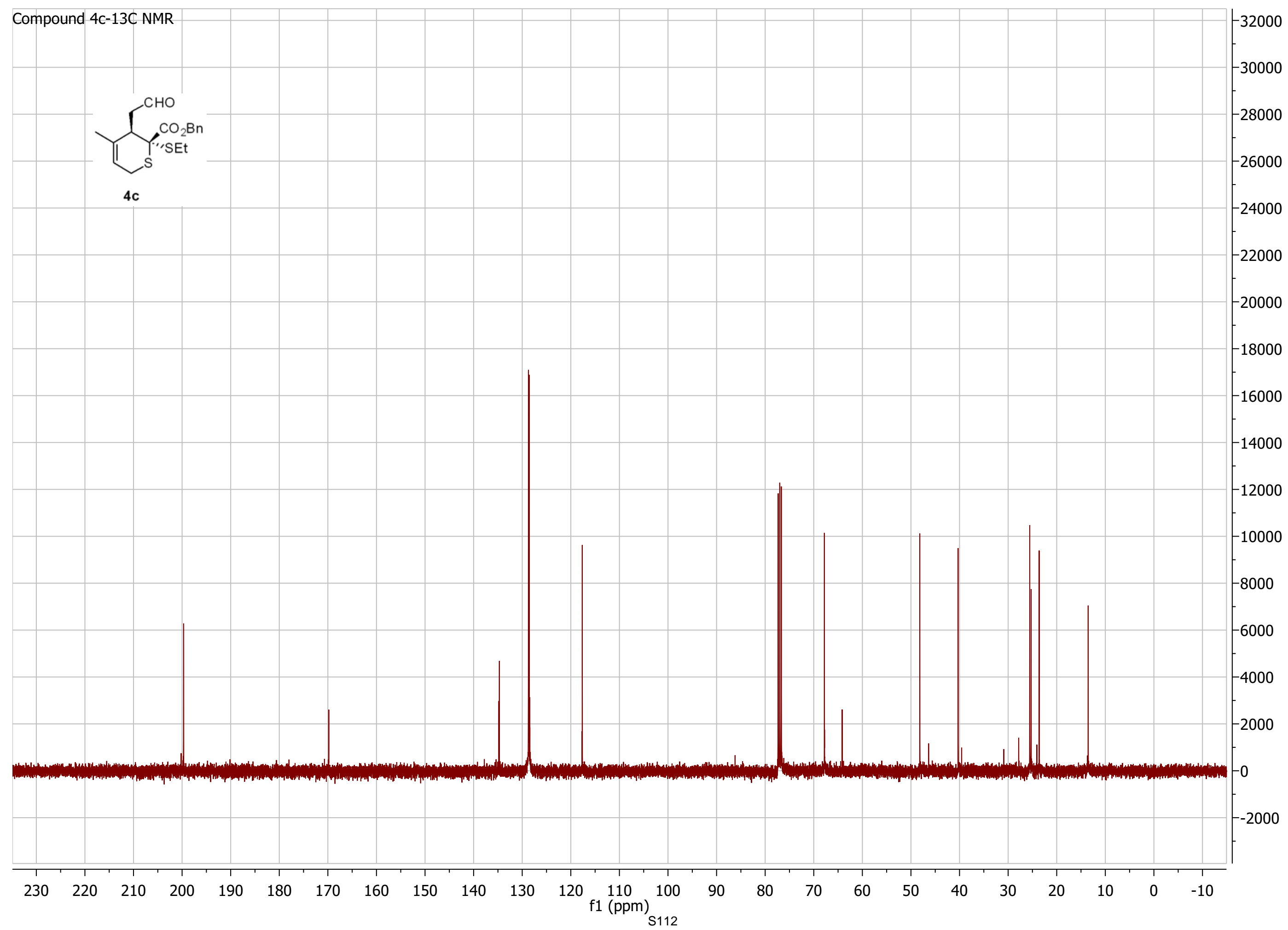




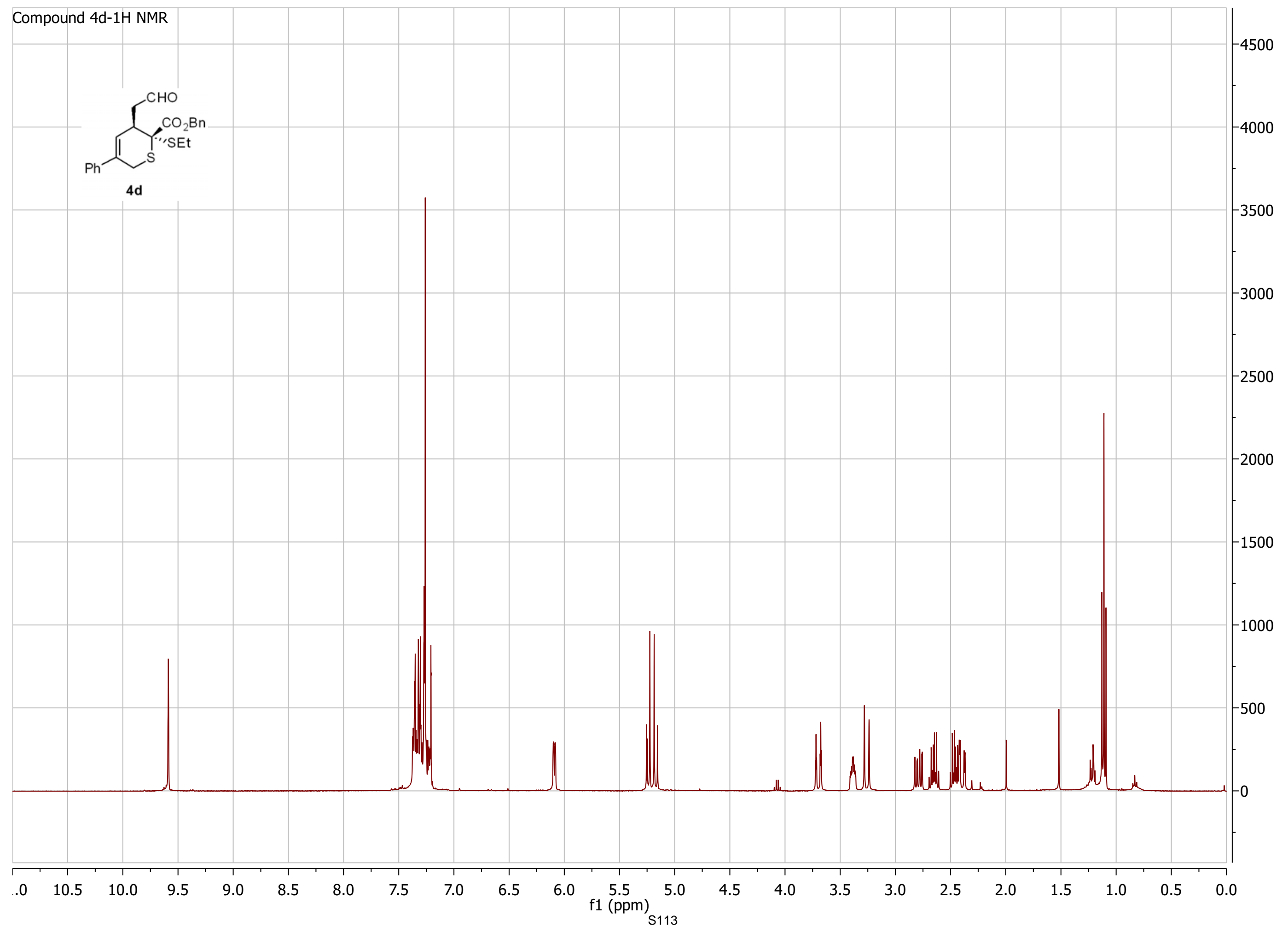




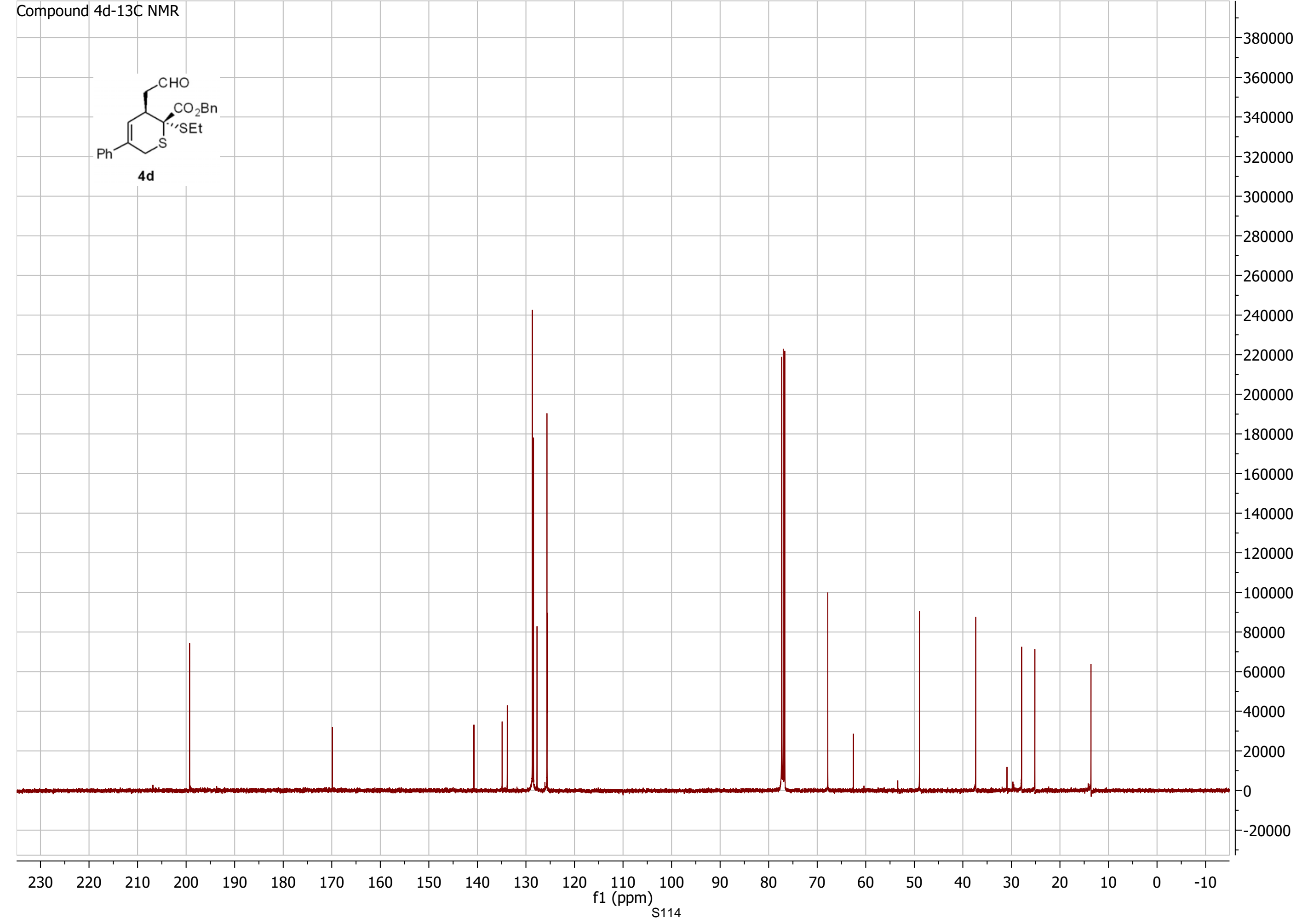




\section{Compound 4e-1H NMR}

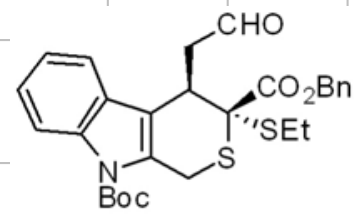

$4 e$ 


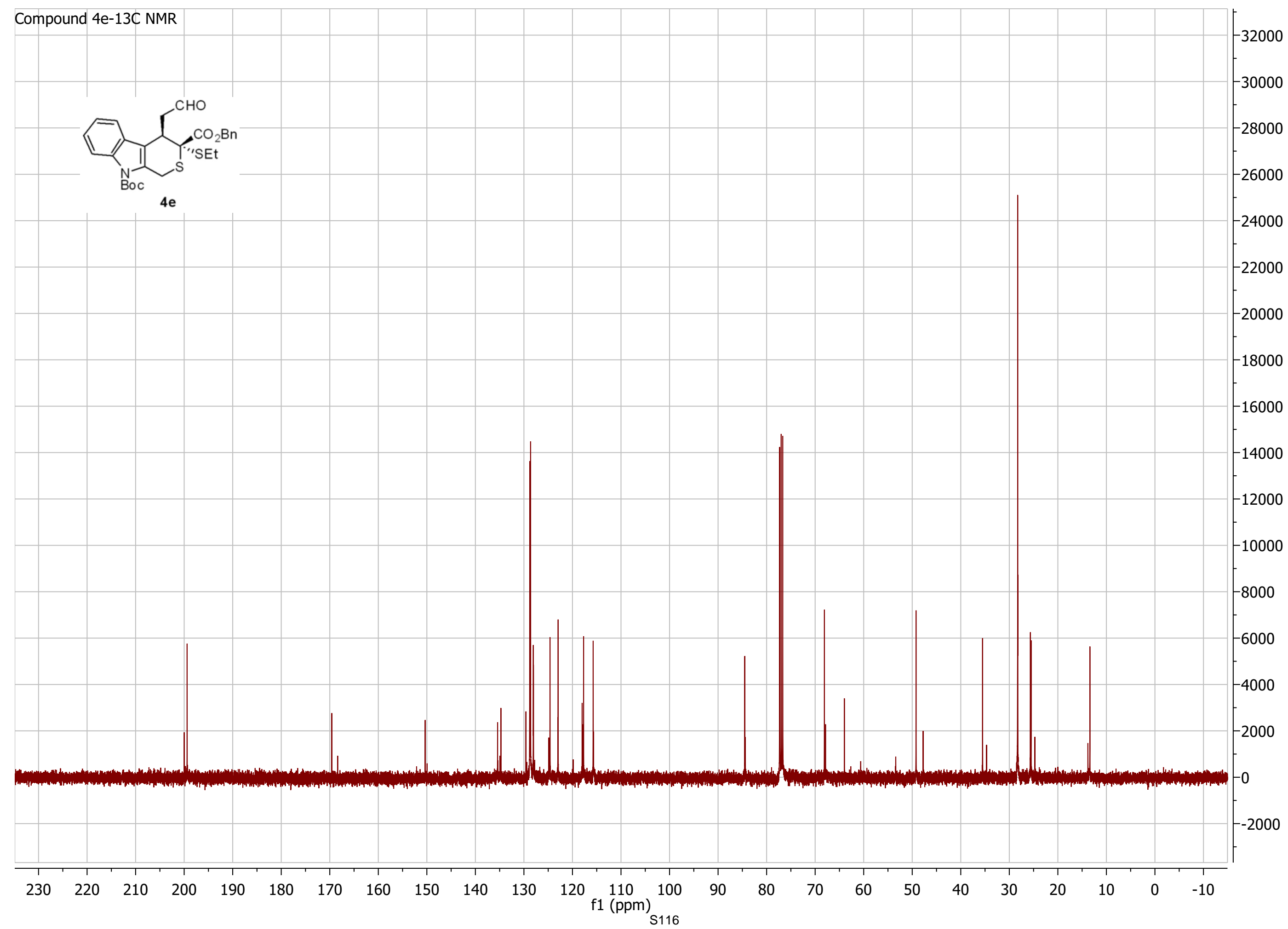




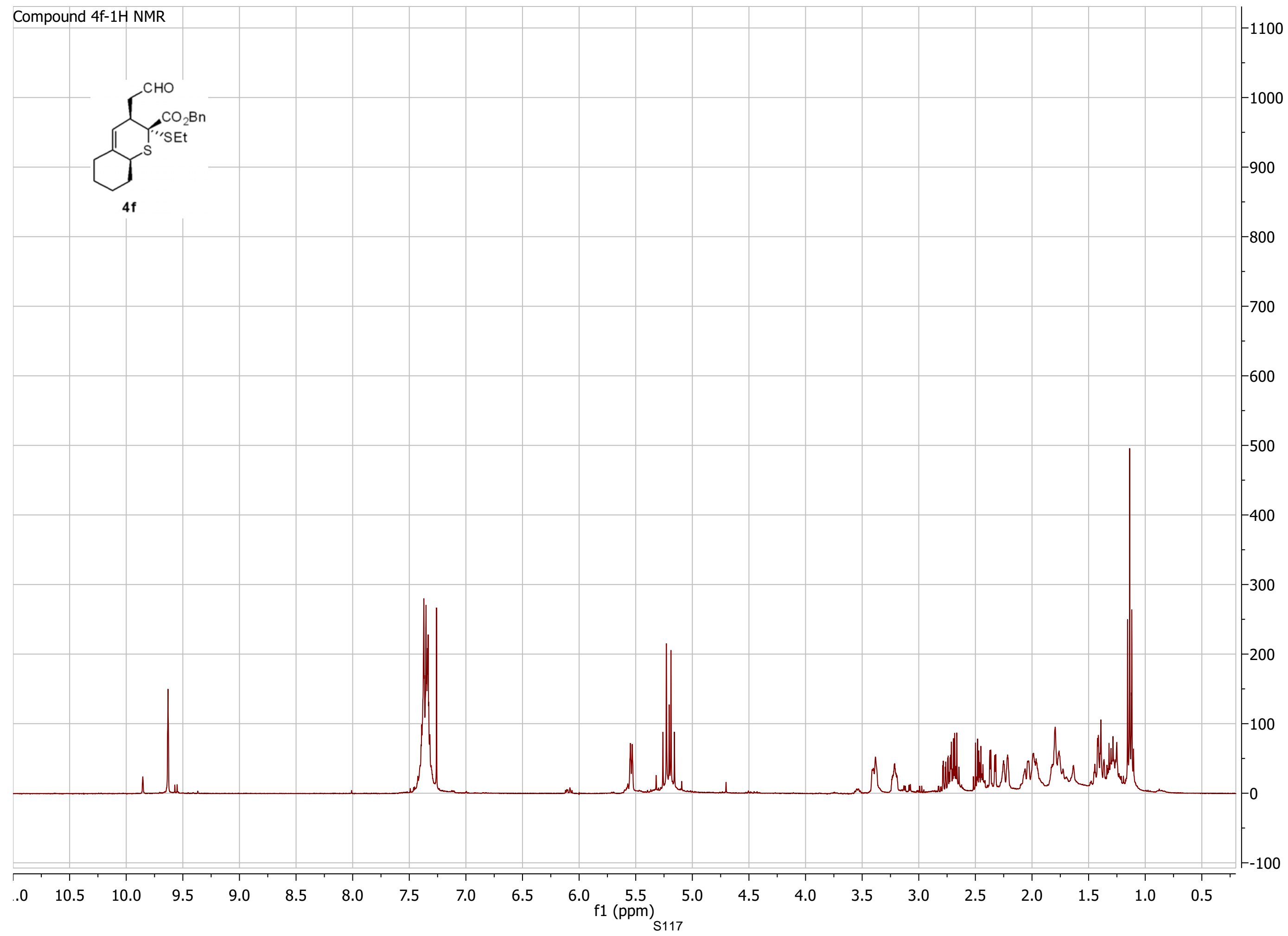




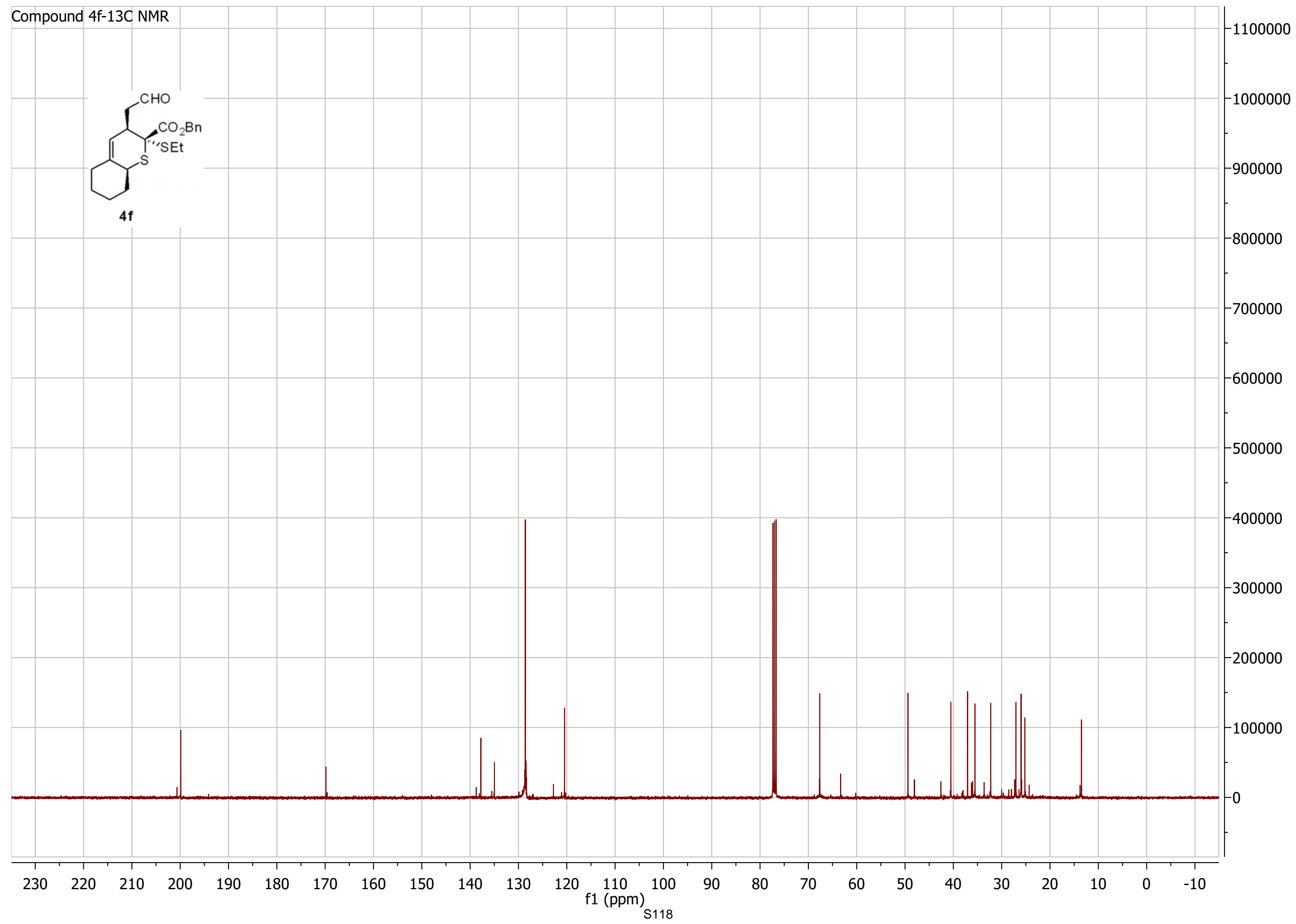




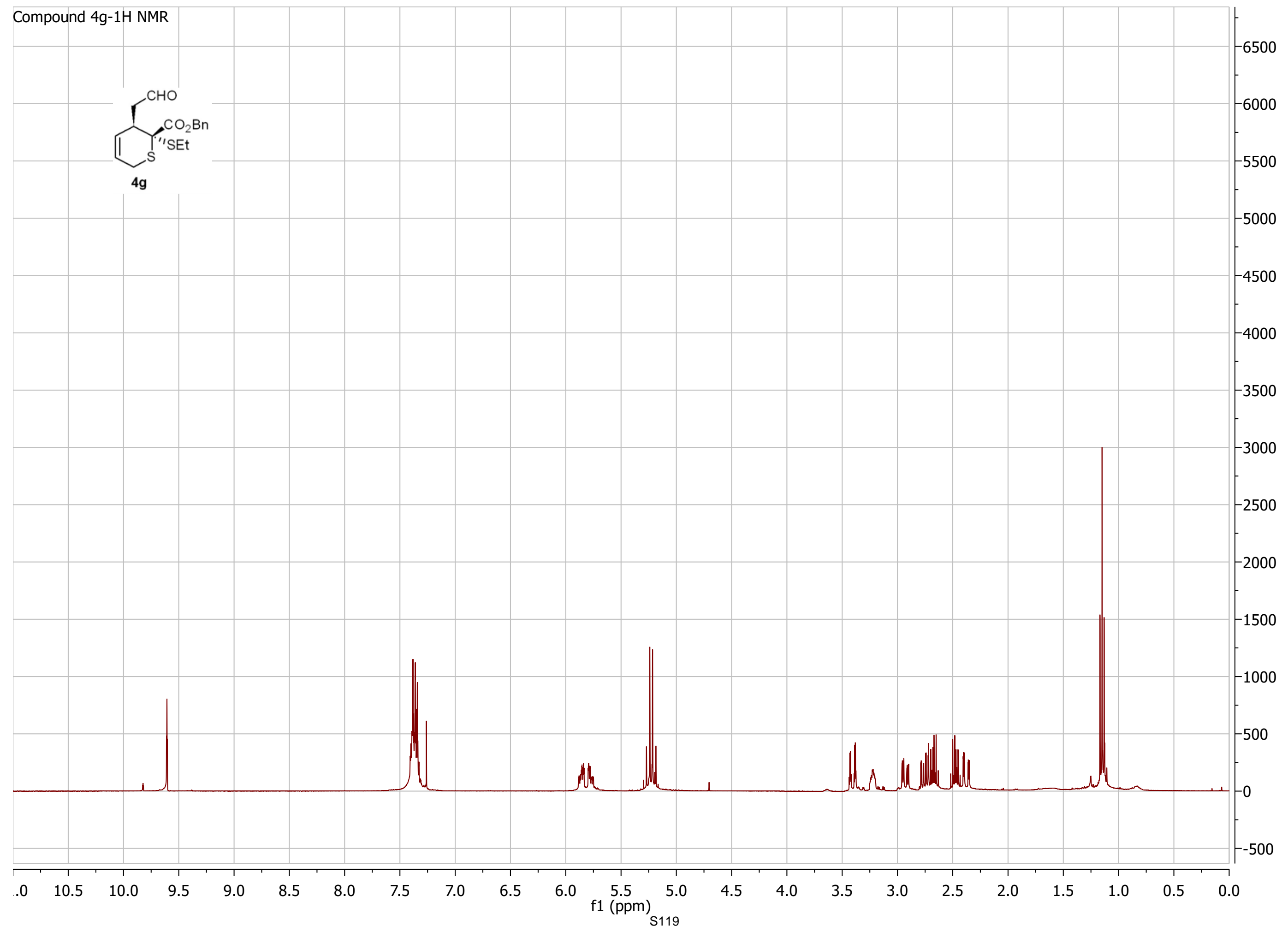




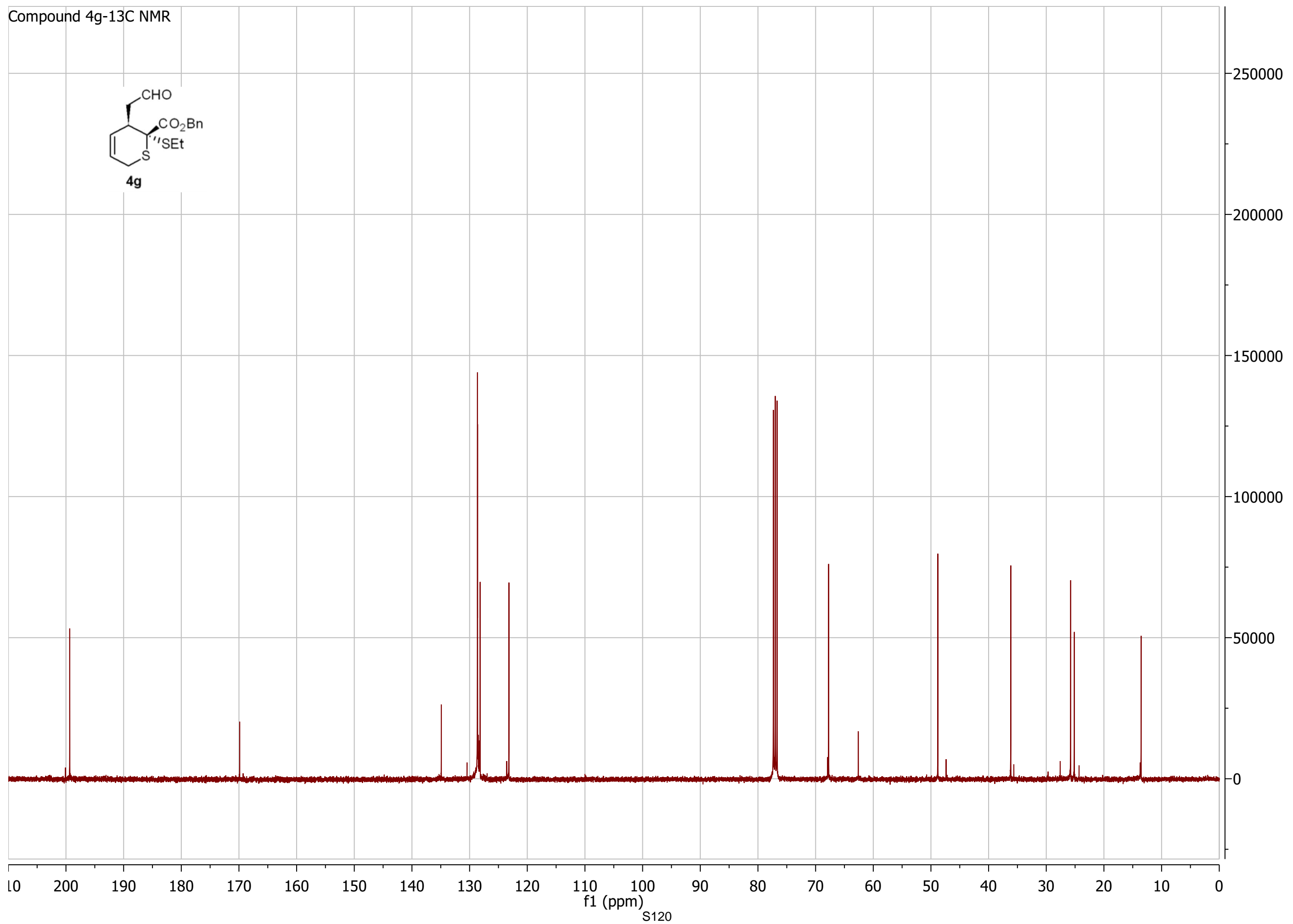




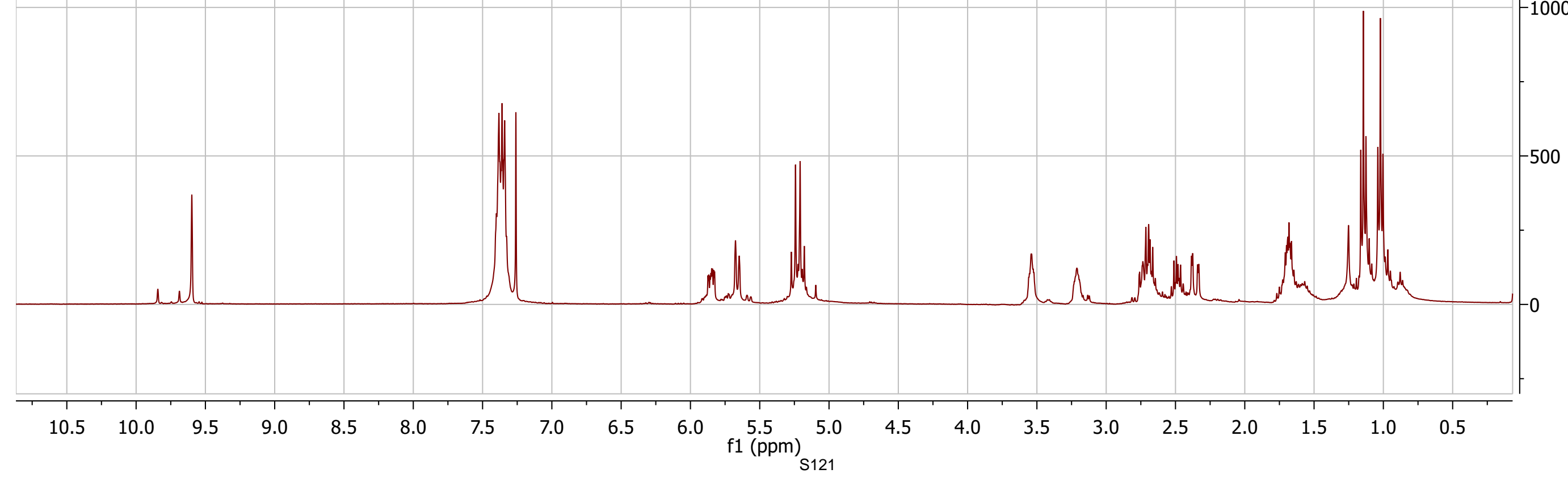




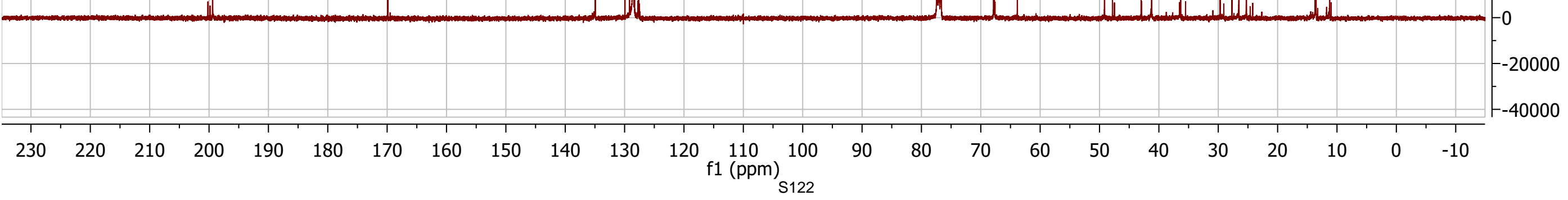




\section{Compound 4i-1H NMR}
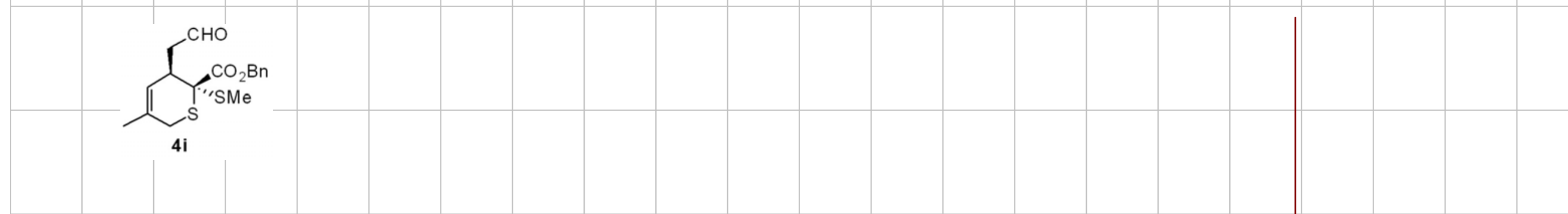

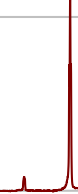

$\begin{array}{llllllllll}4.5 & 4.0 & 3.5 & 3.0 & 2.5 & 2.0 & 1.5 & 1.0 & 0.5 & 0.0\end{array}$




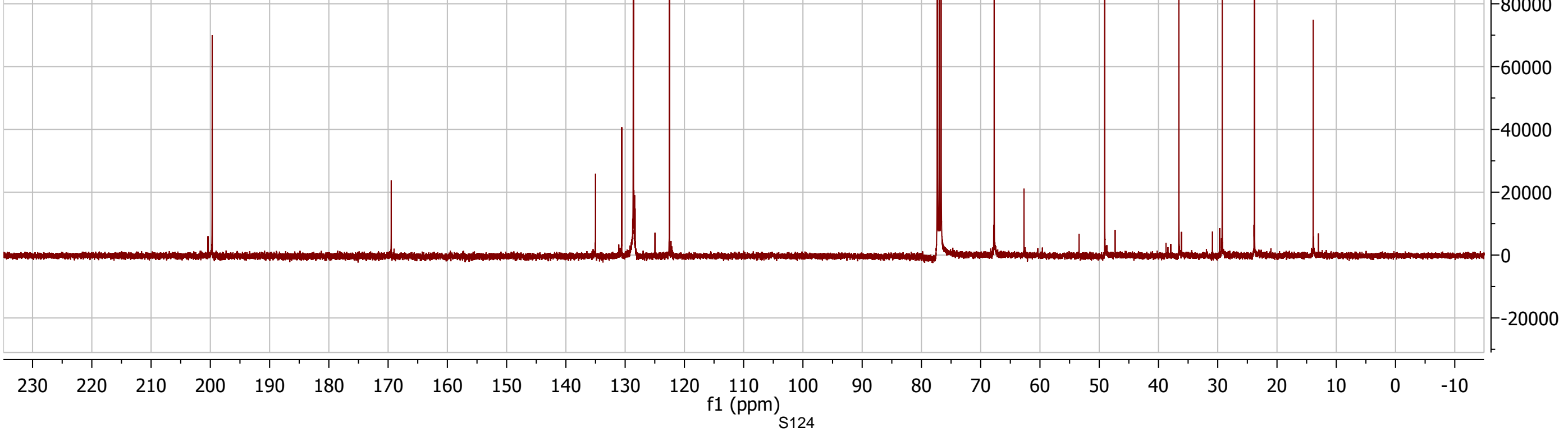




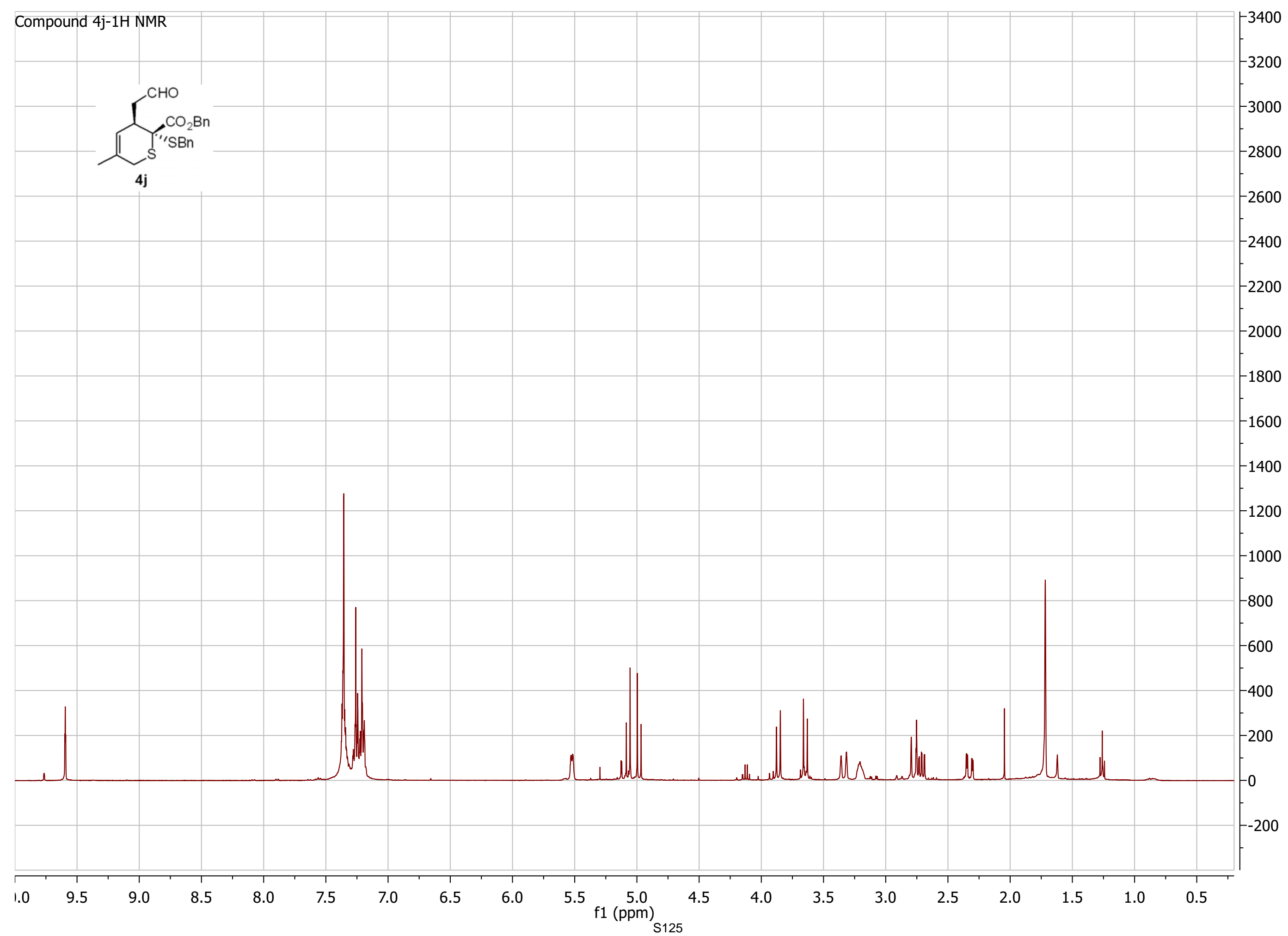




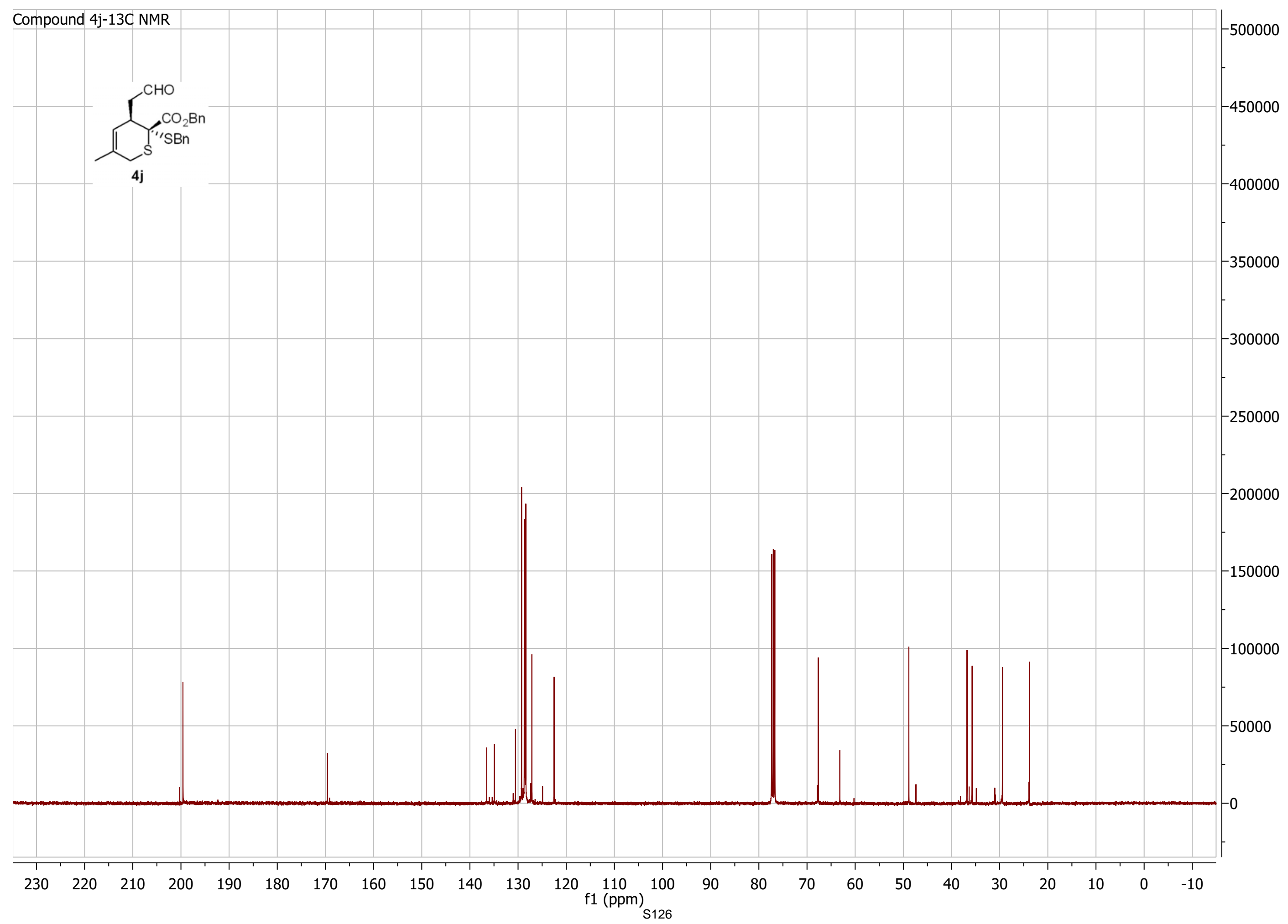



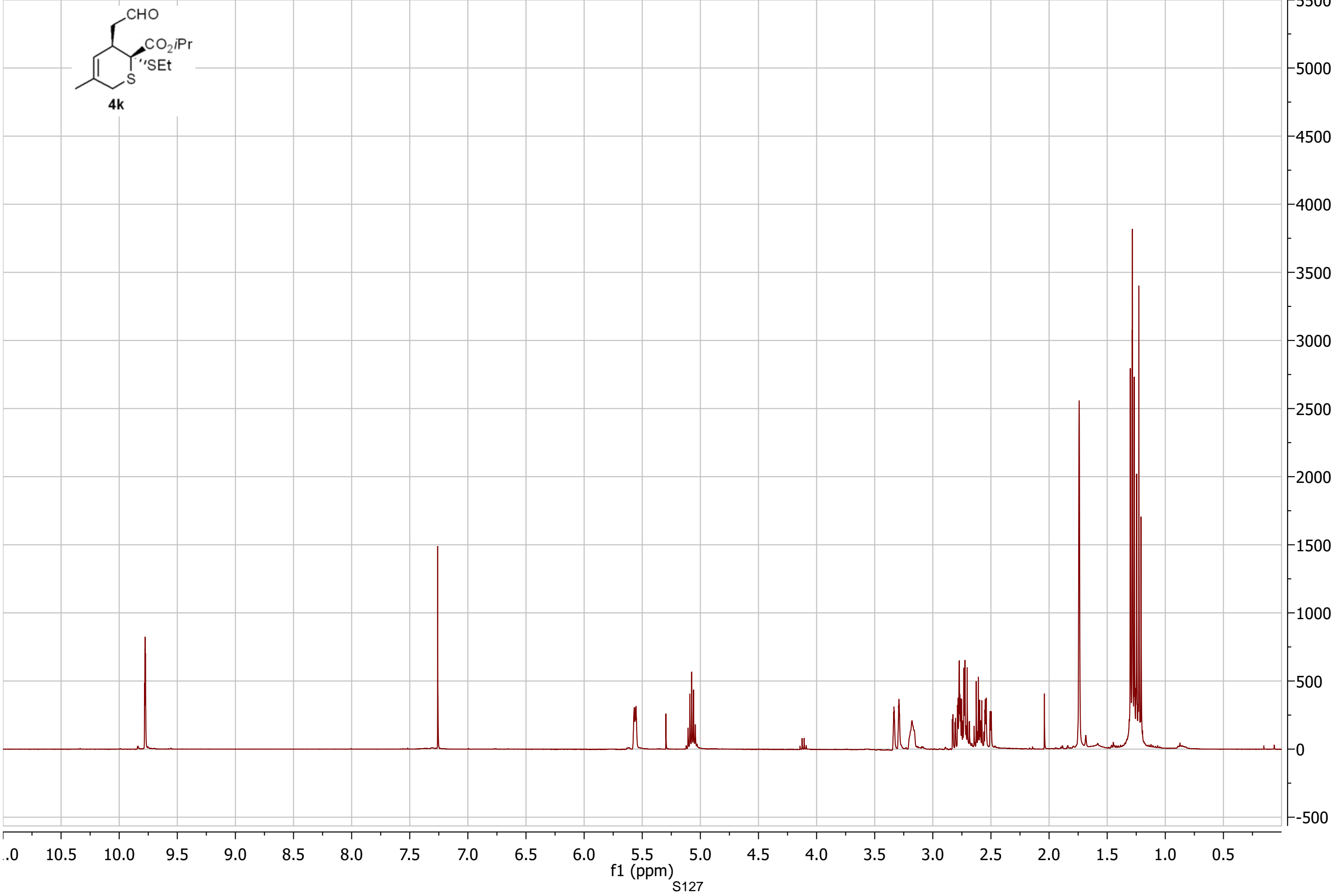


\section{Compound 4k-13C $M R$}

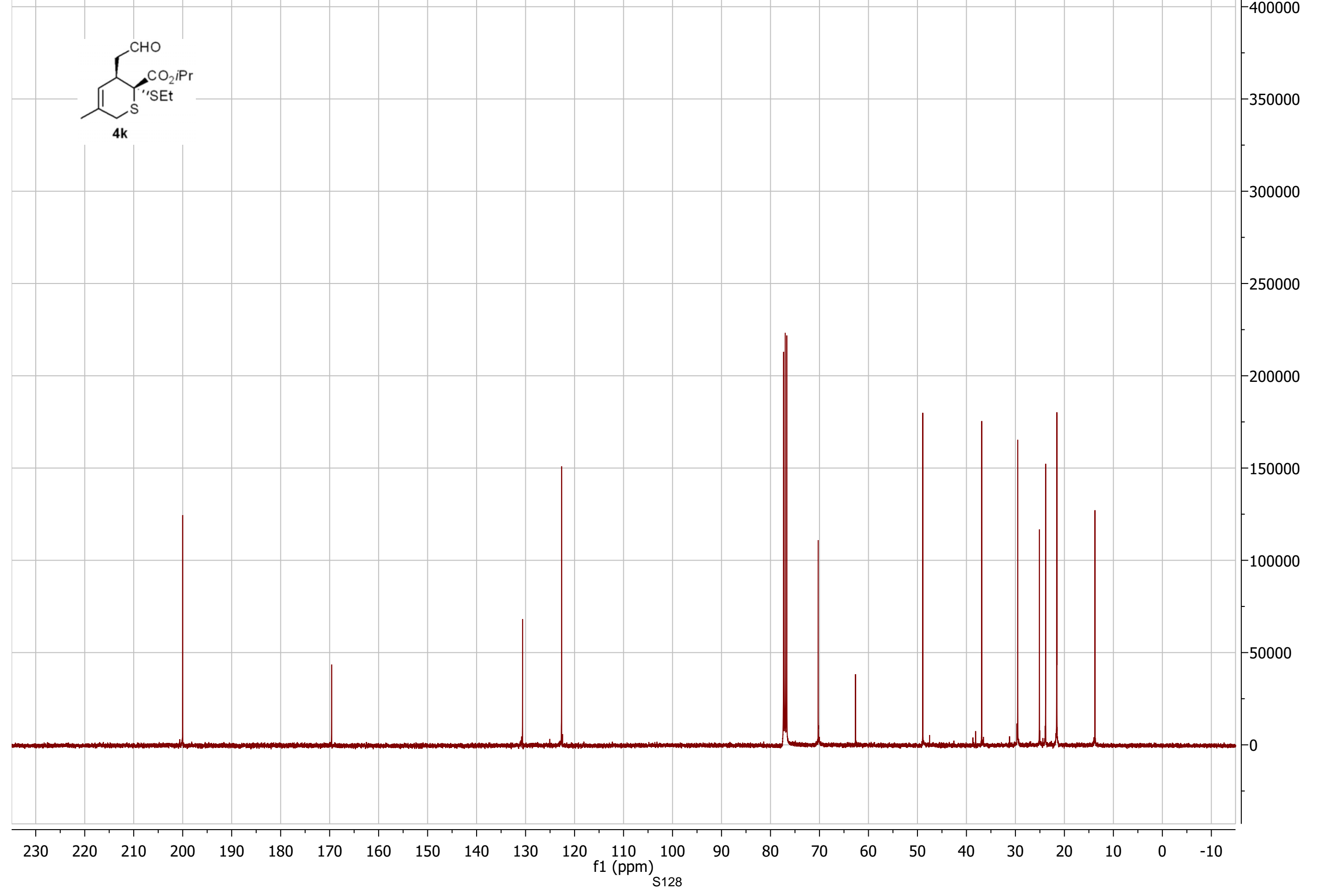




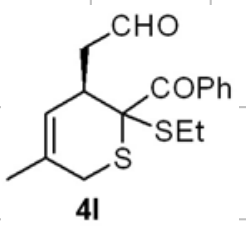




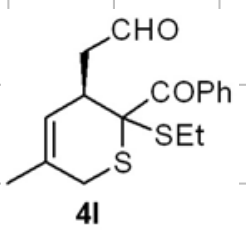




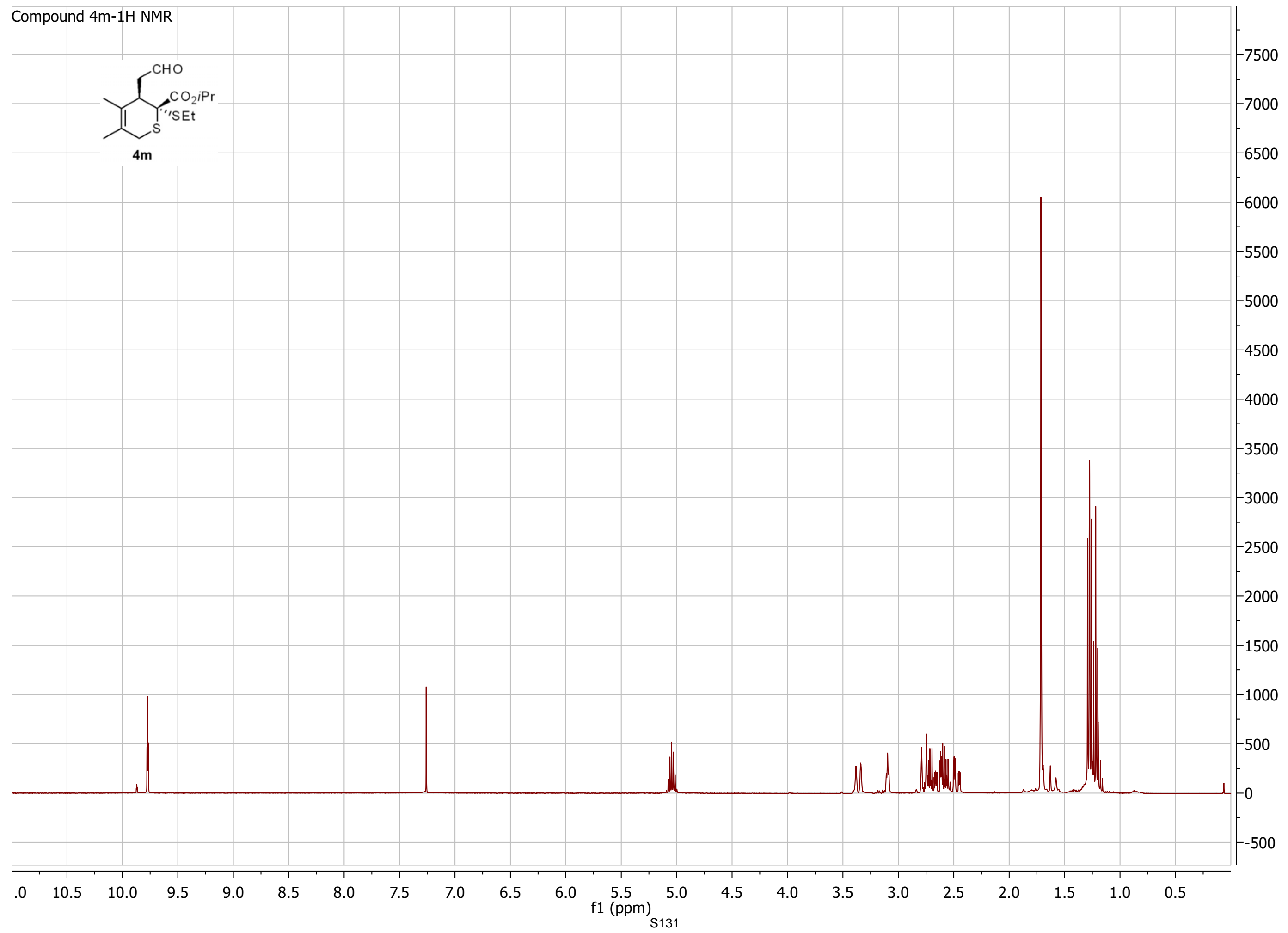




\section{Compound 4m-13C NMR}

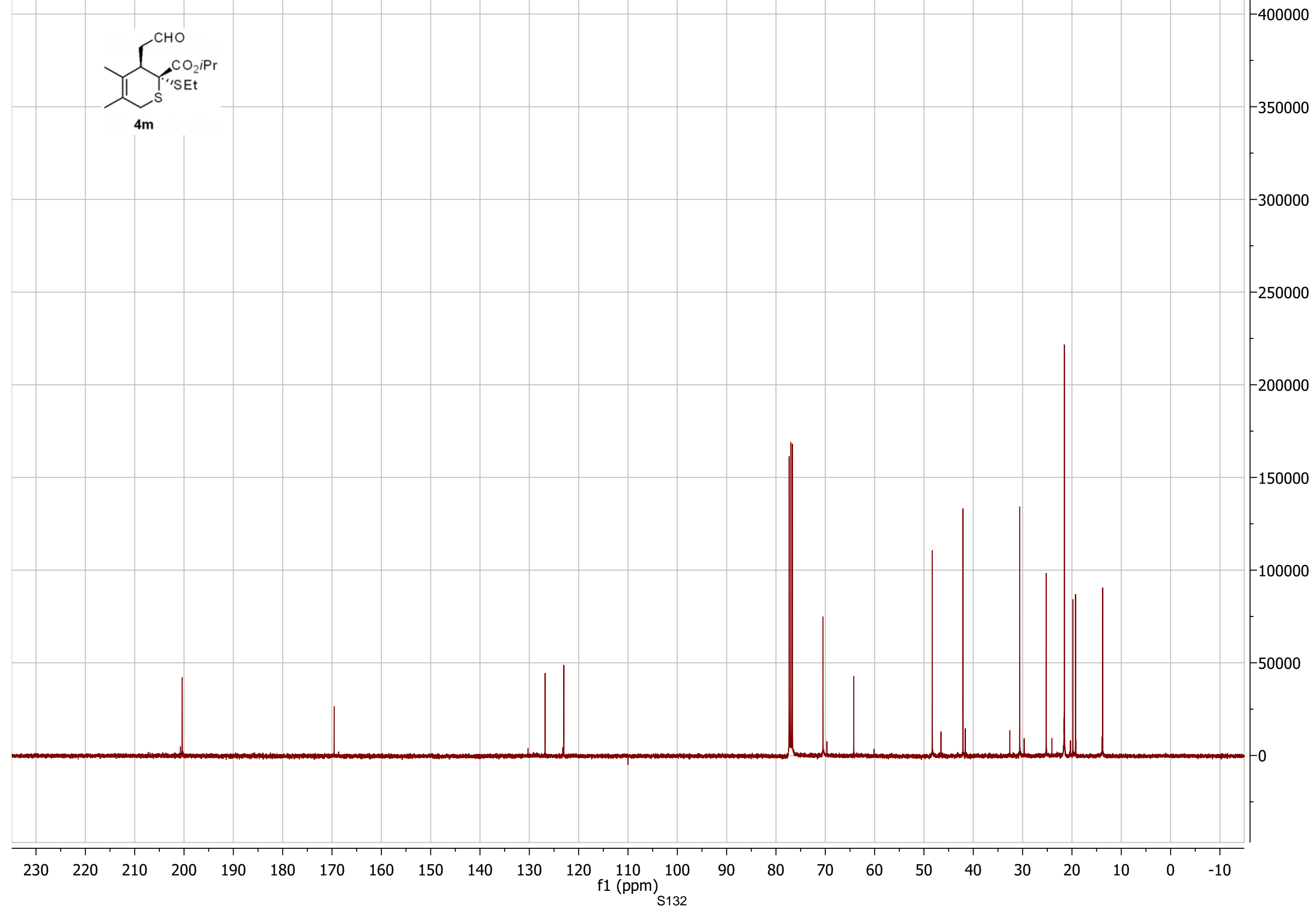




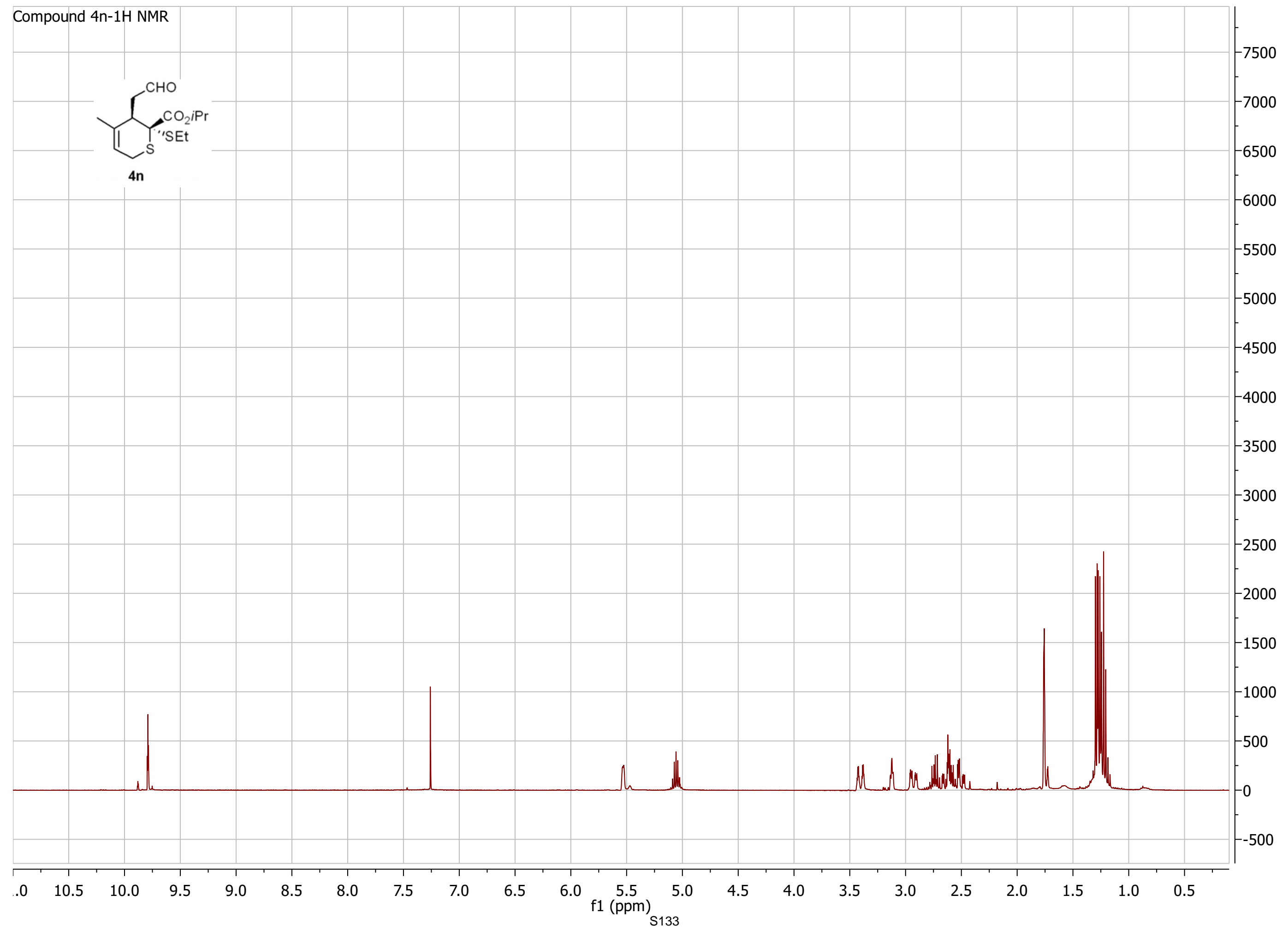




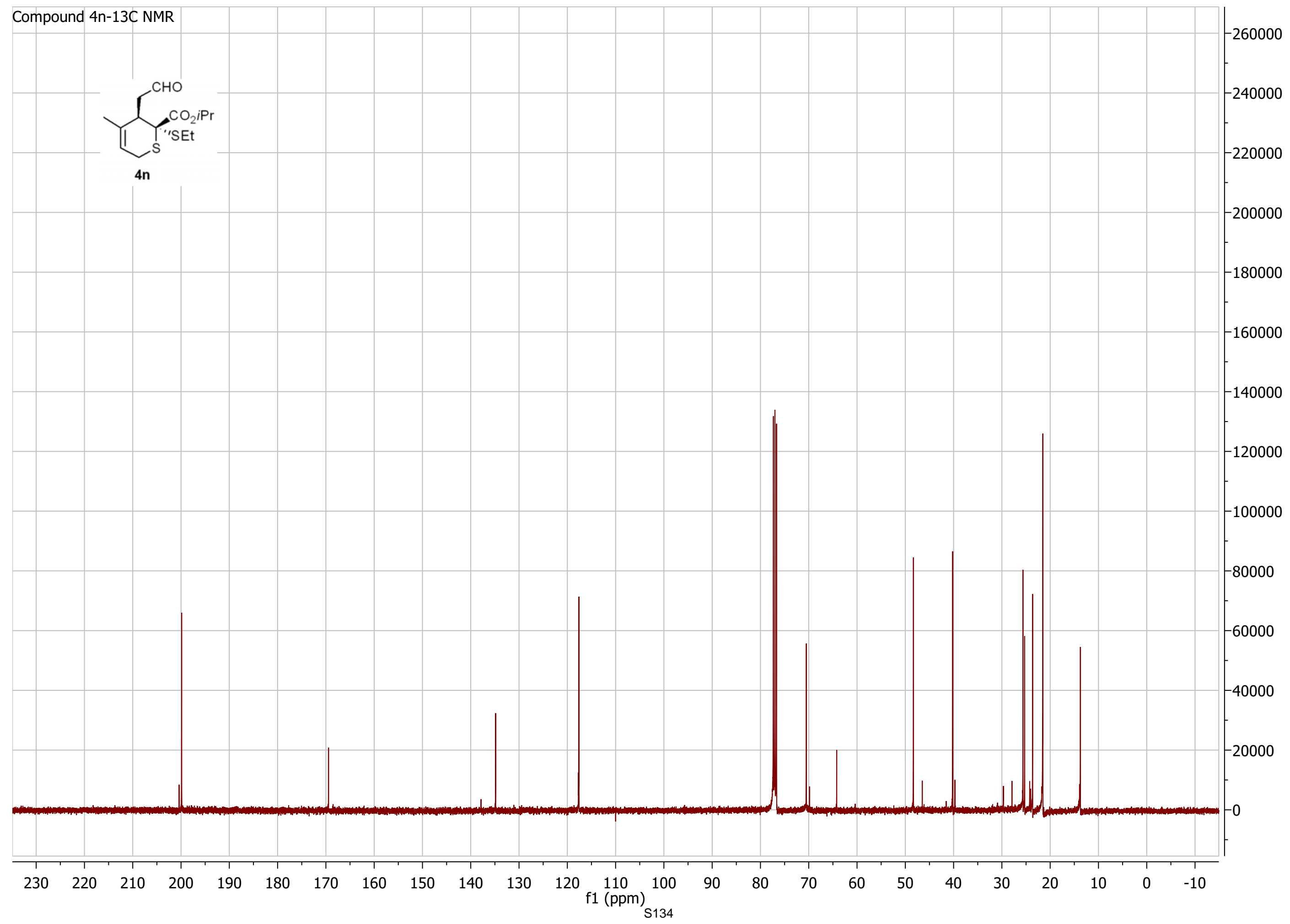




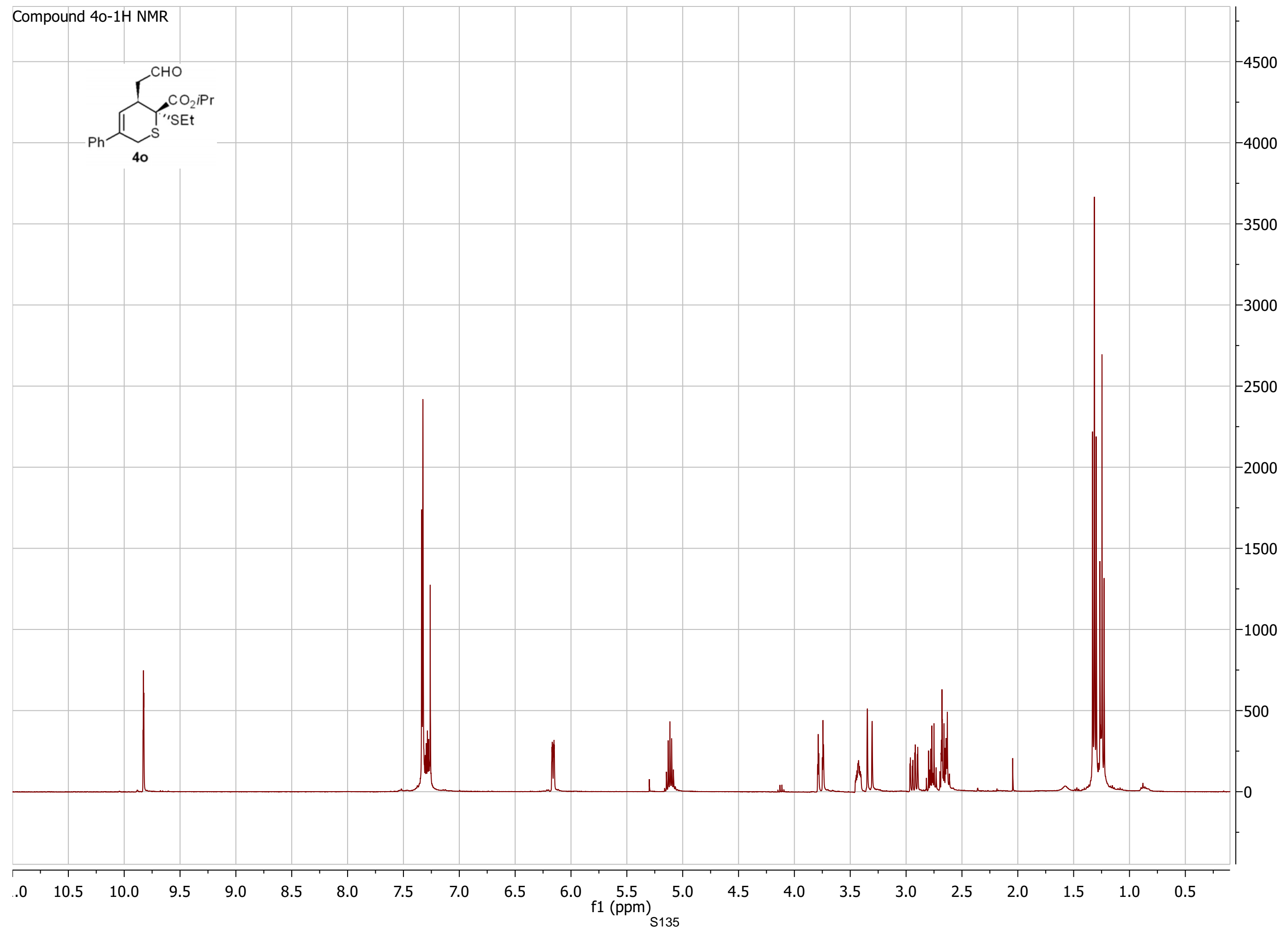




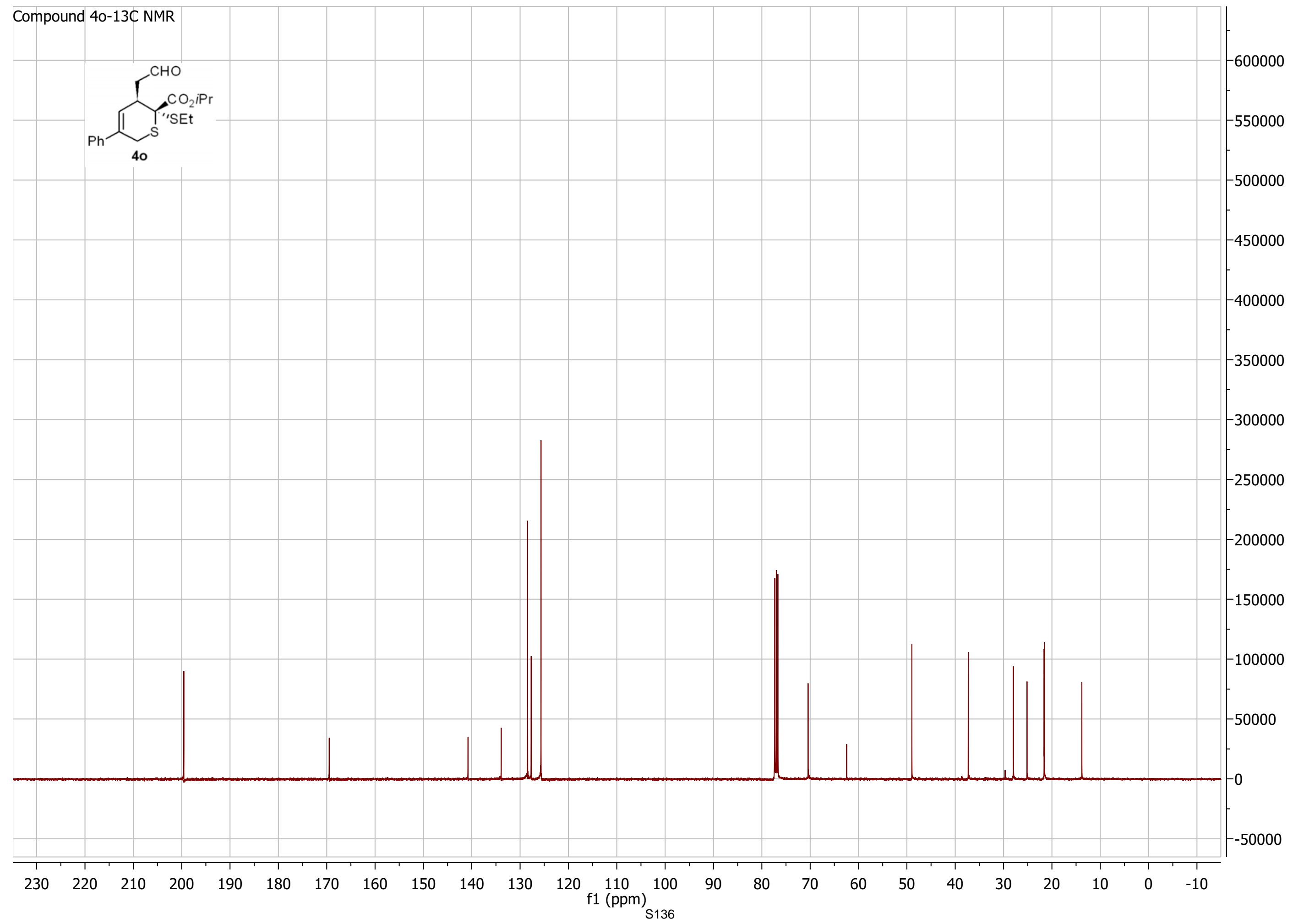




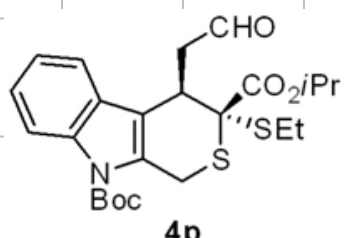

$4 p$

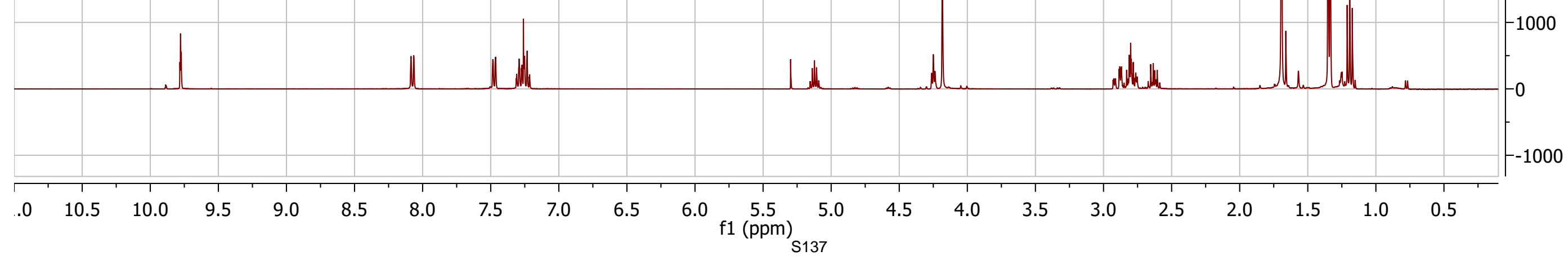




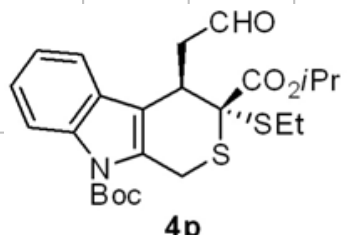

$-550000$

$-500000$

$-450000$

$-400000$

$-350000$

$-300000$

250000

$-200000$

$-150000$

$-100000$

$-50000$

$\begin{array}{llllllllllll}230 & 220 & 210 & 200 & 190 & 180 & 170 & 160 & 150 & 140 & 130 & 120 \underset{\mathrm{f} 1(\mathrm{ppm})}{110})_{\mathrm{S} 138}\end{array}$

S138 


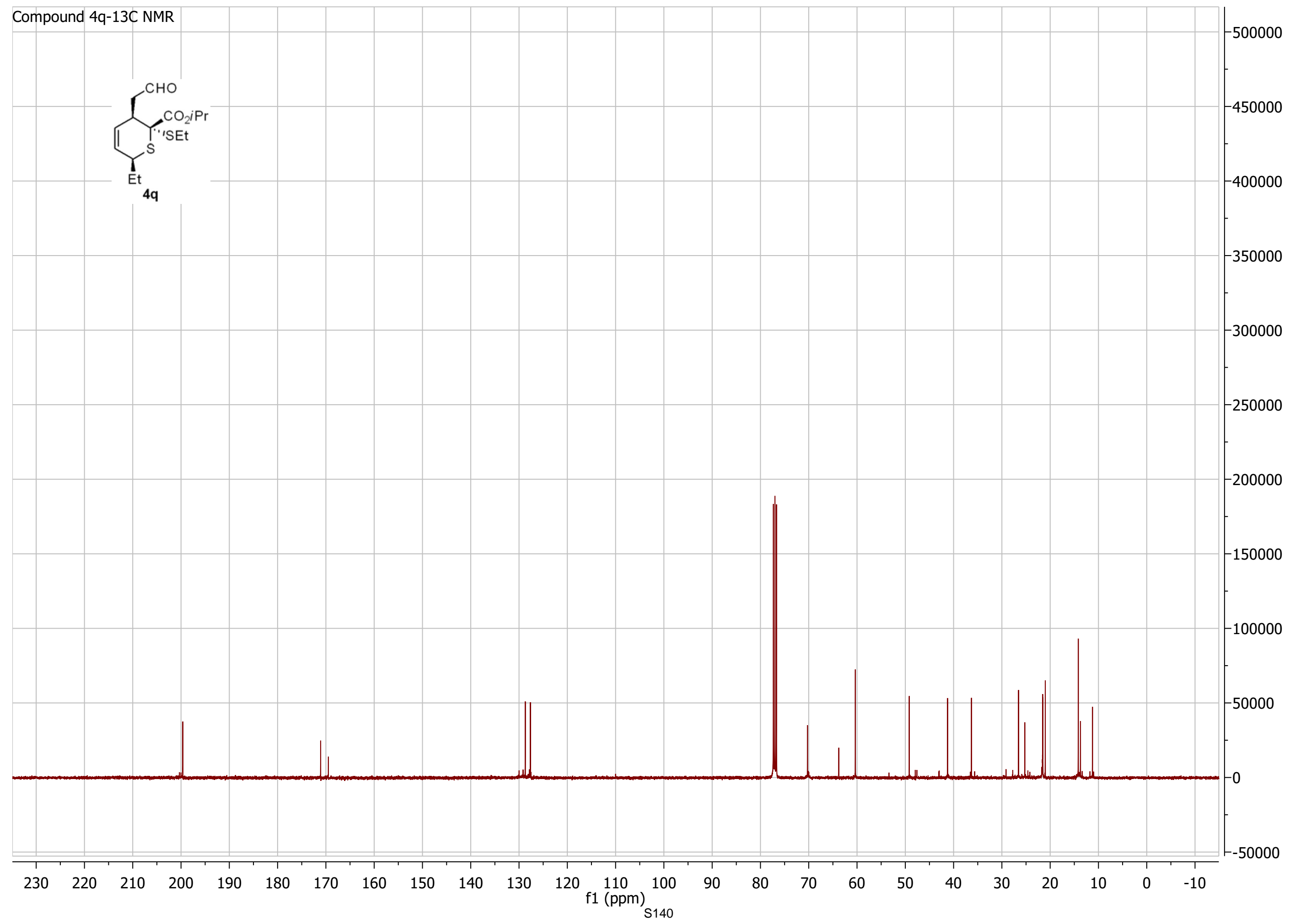




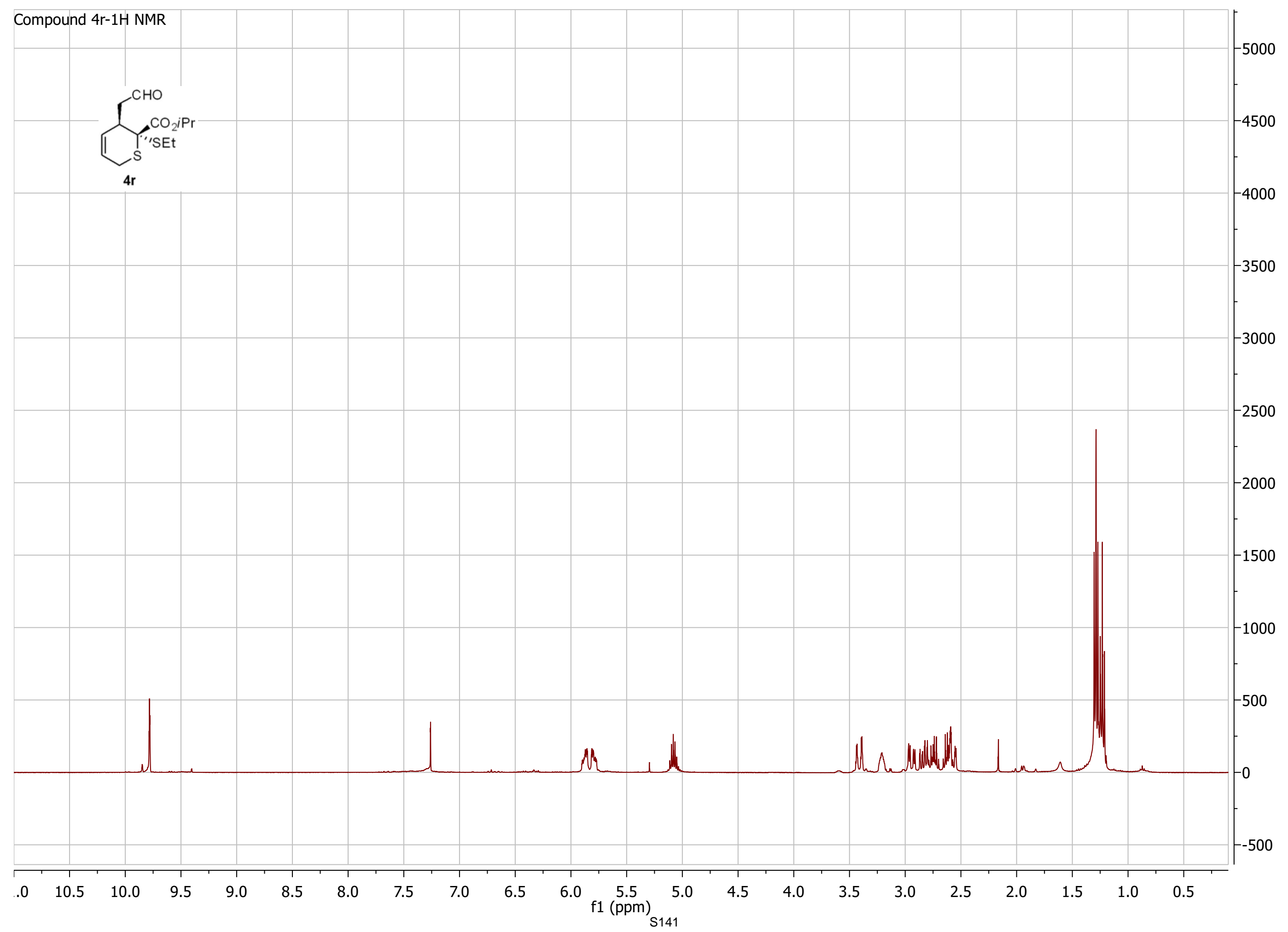




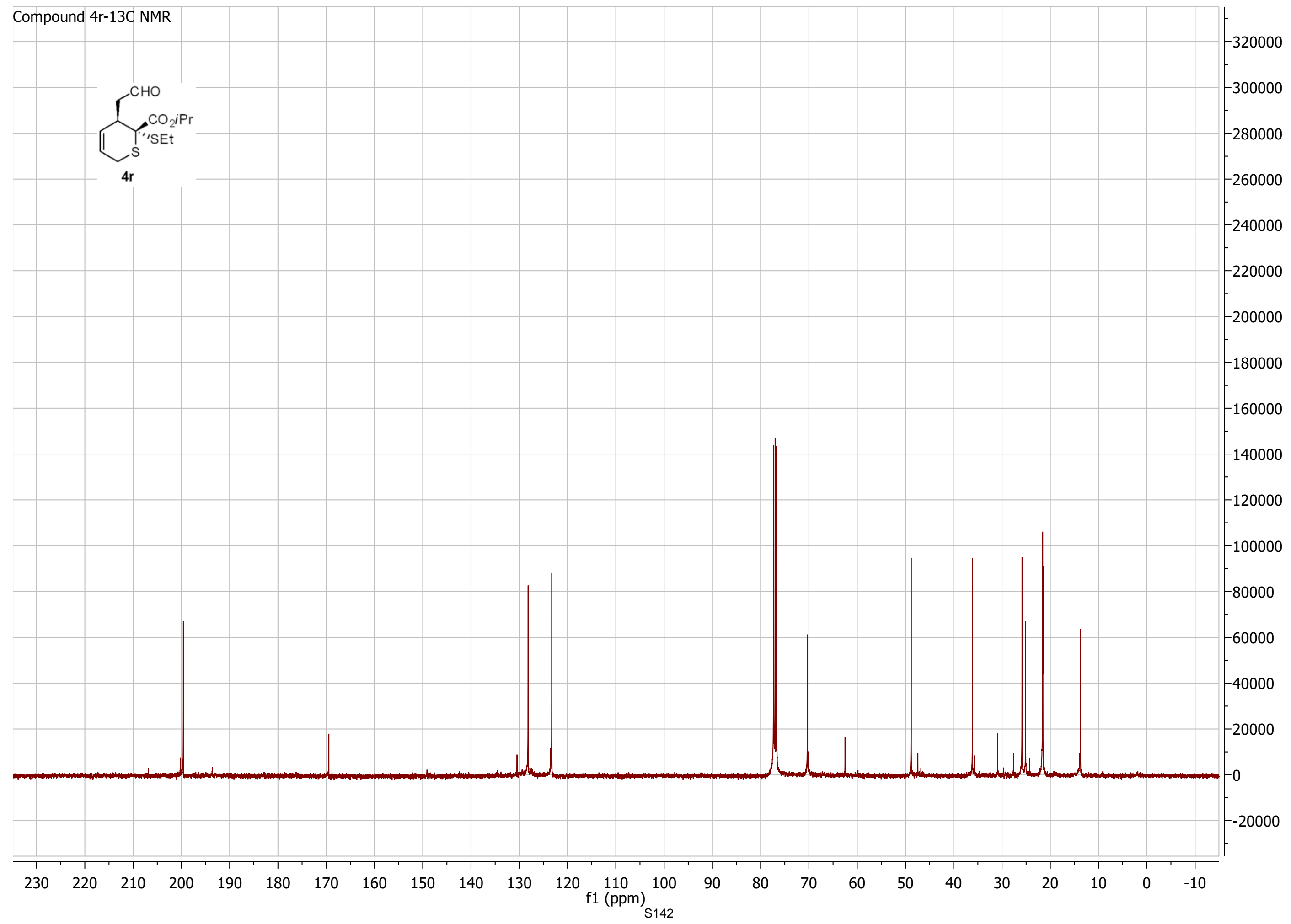




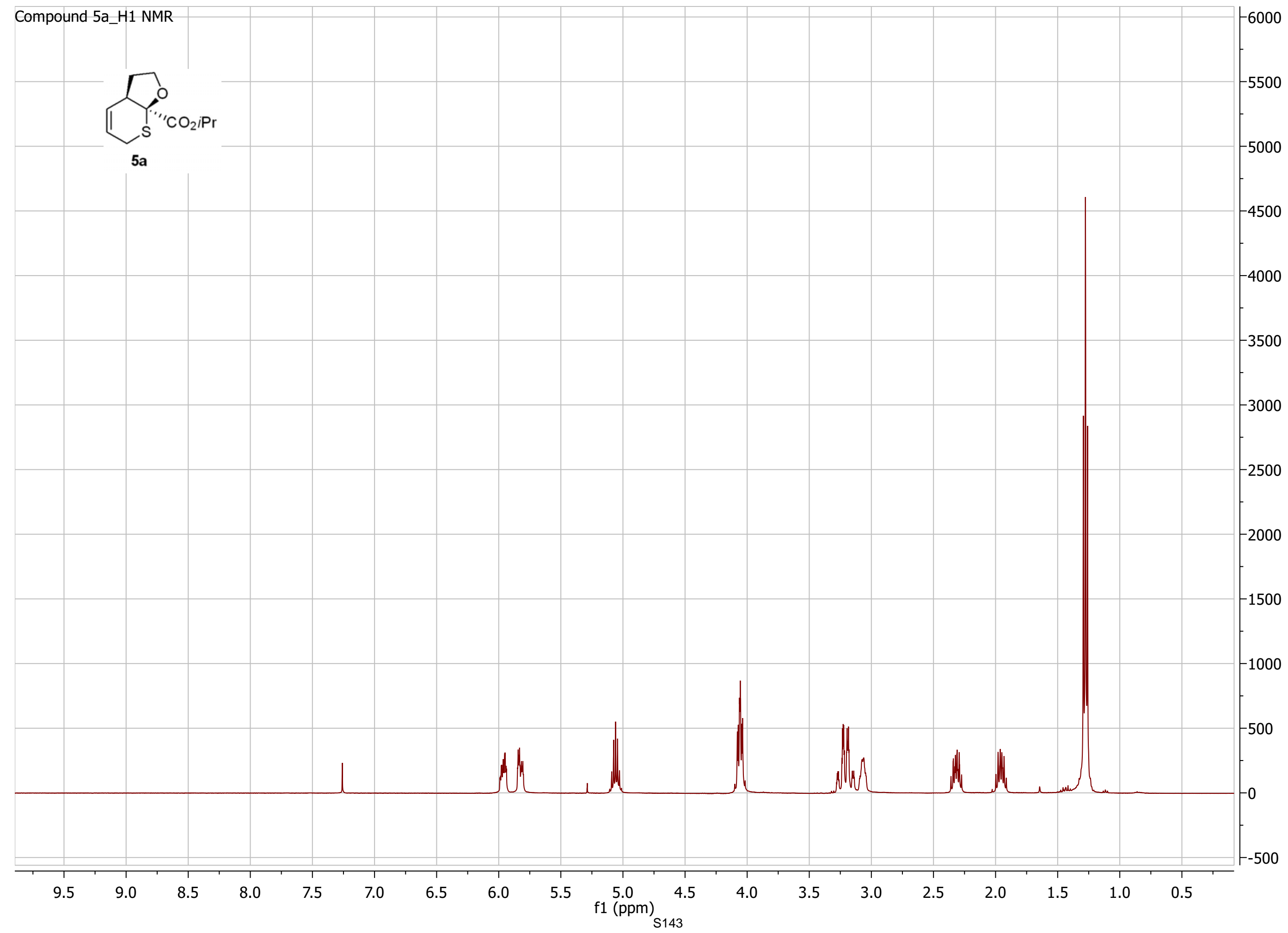




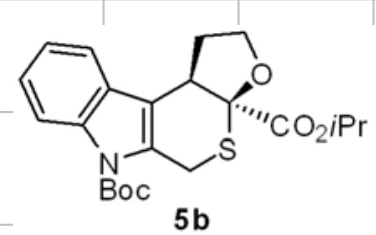




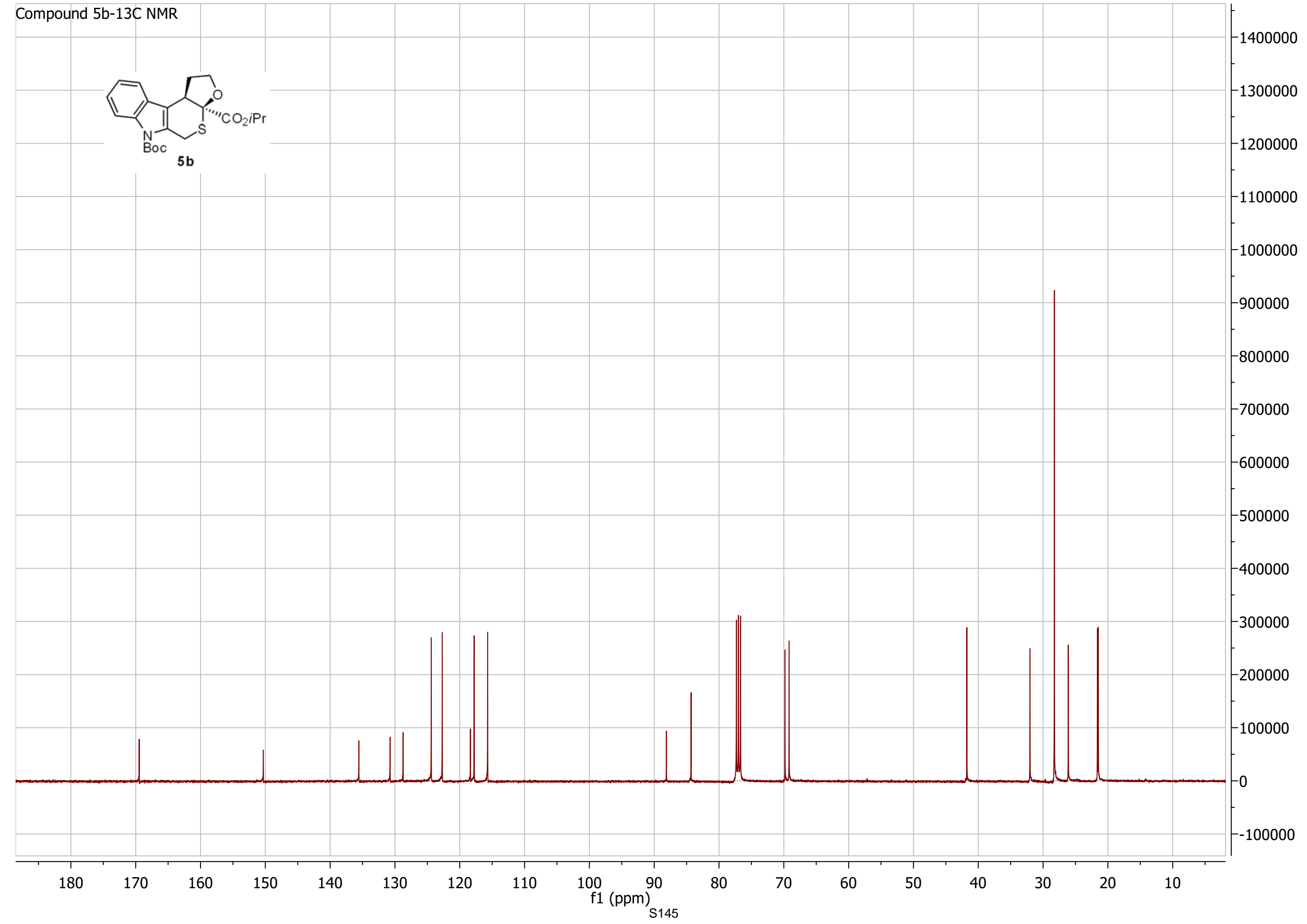




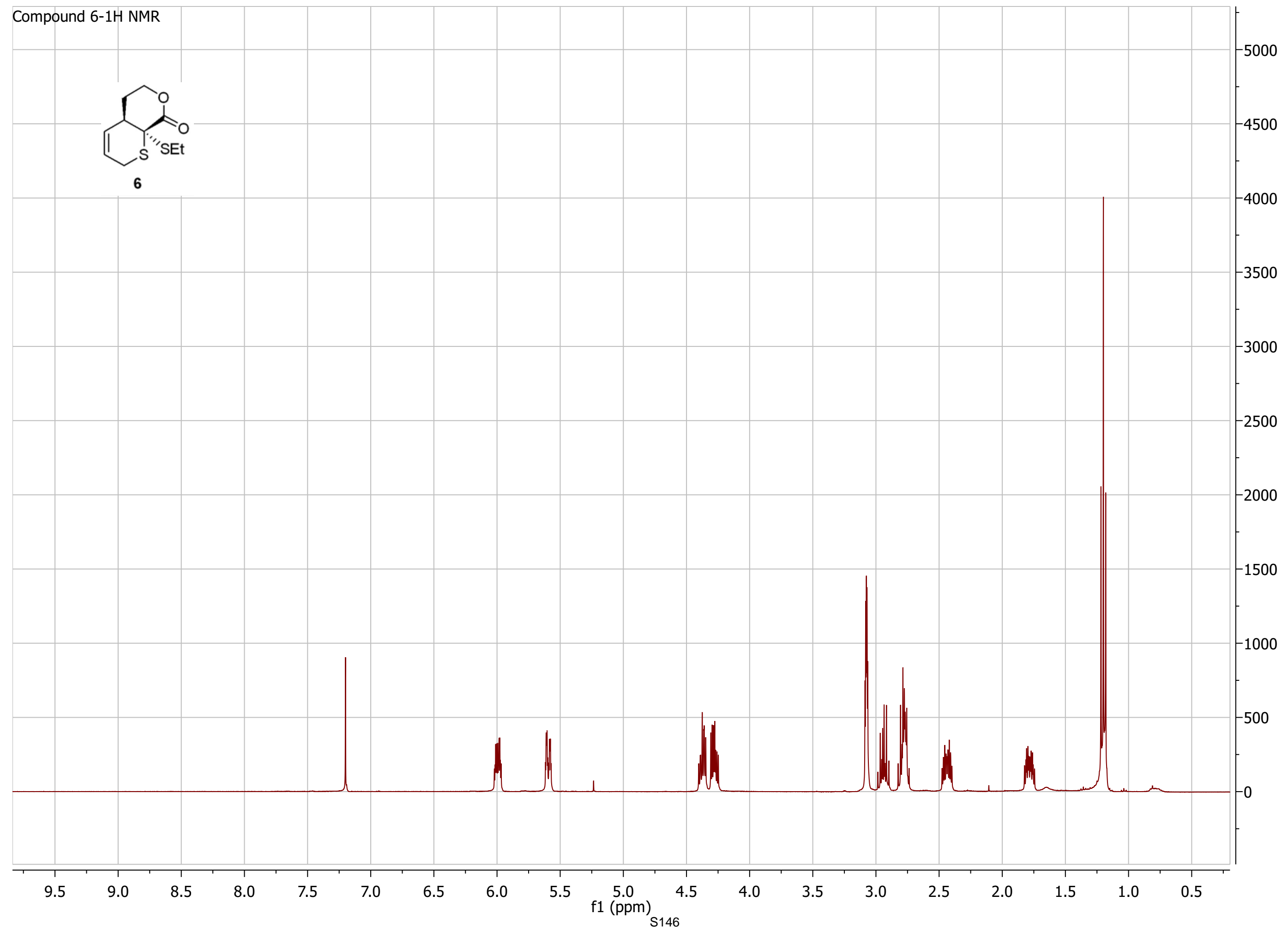




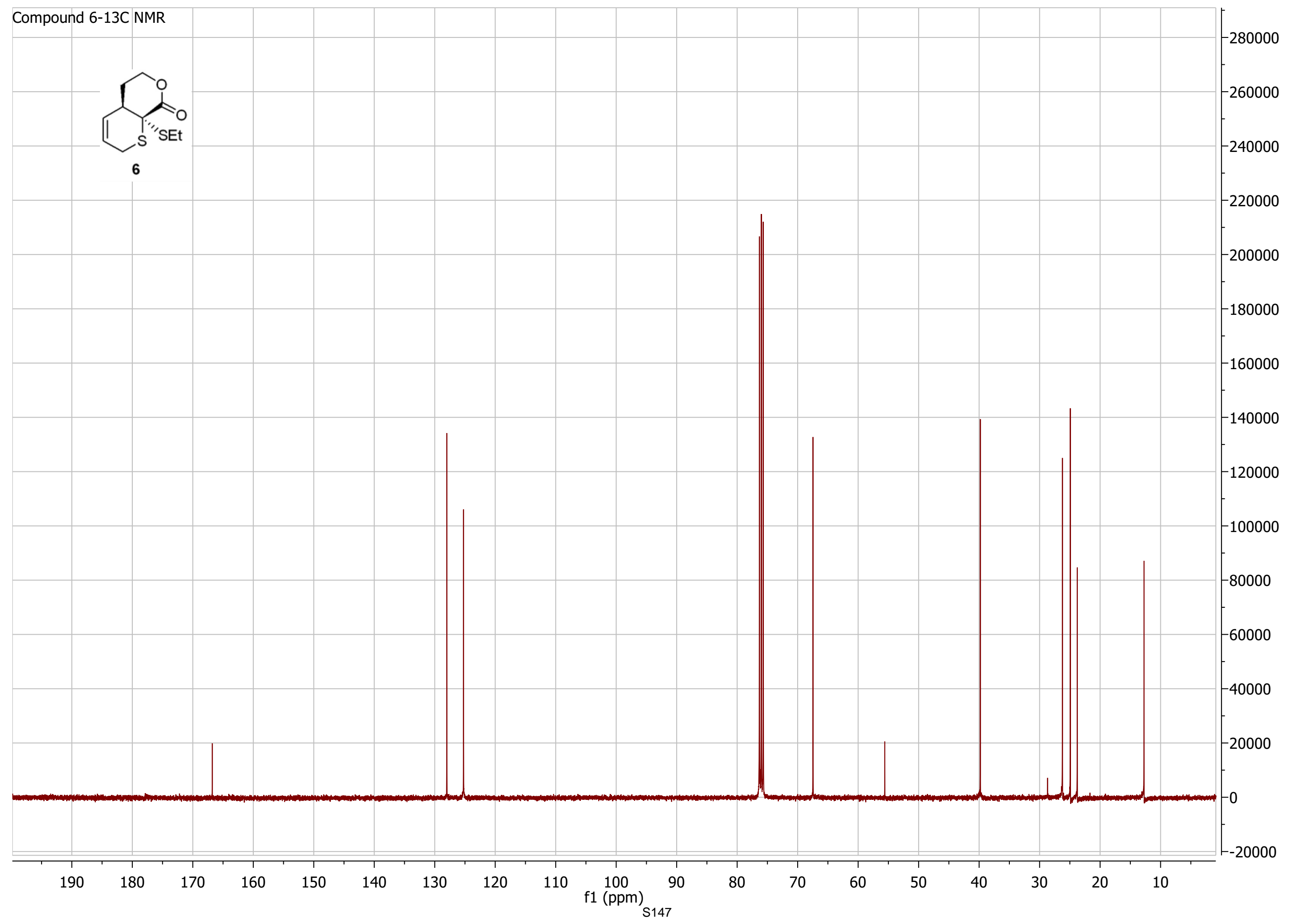


<smiles>[3H]C1CS[C@@]2(COC(C)C)OCCC2C(O)[C@@H]1O</smiles> 


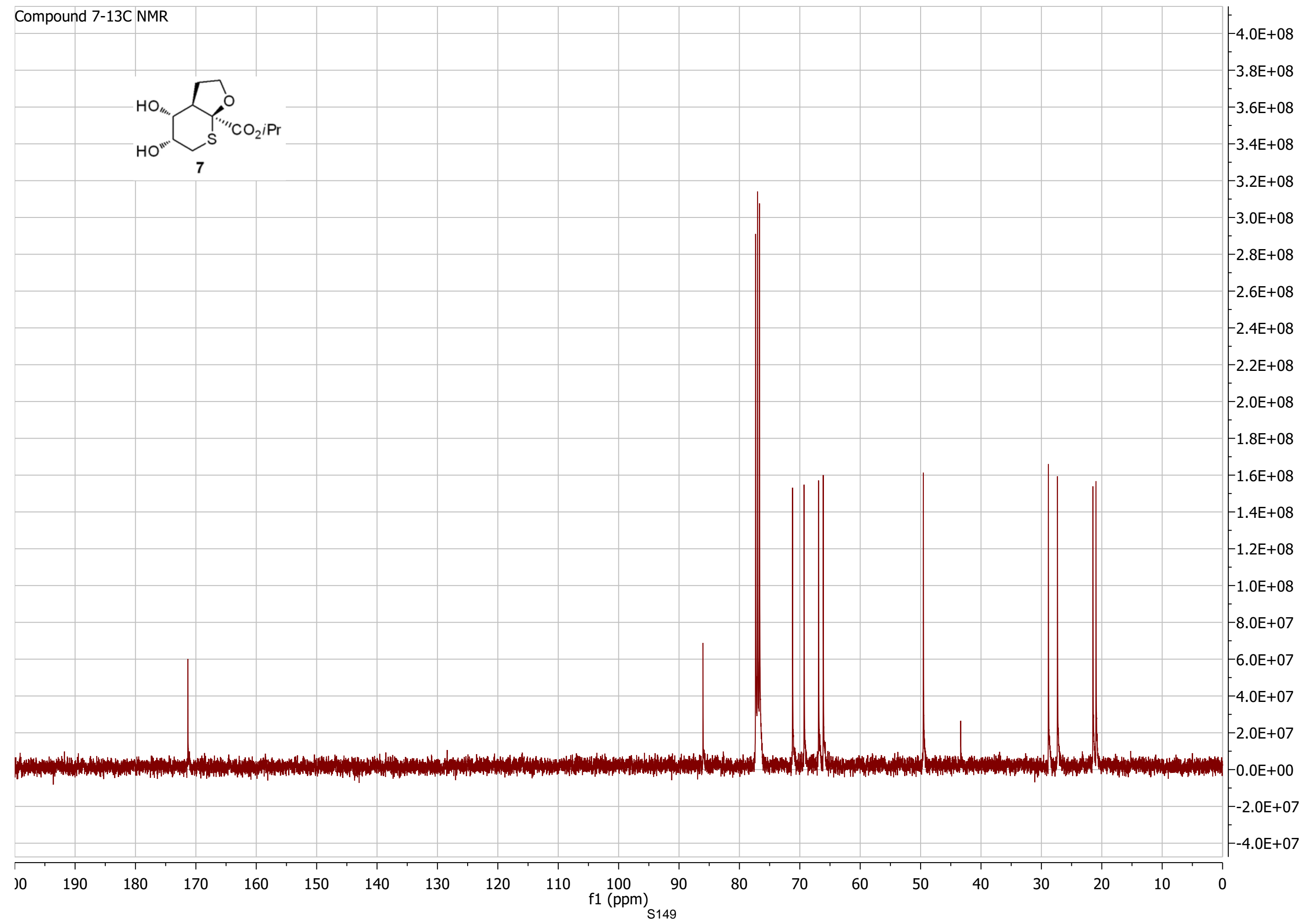




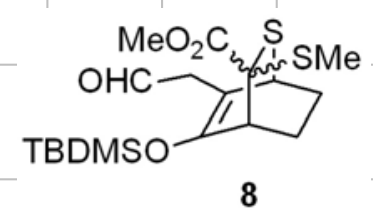

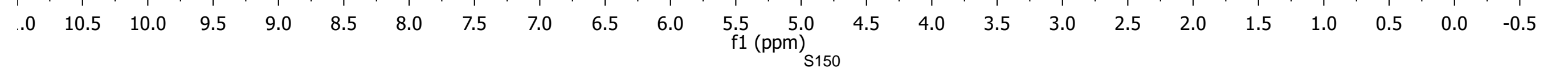




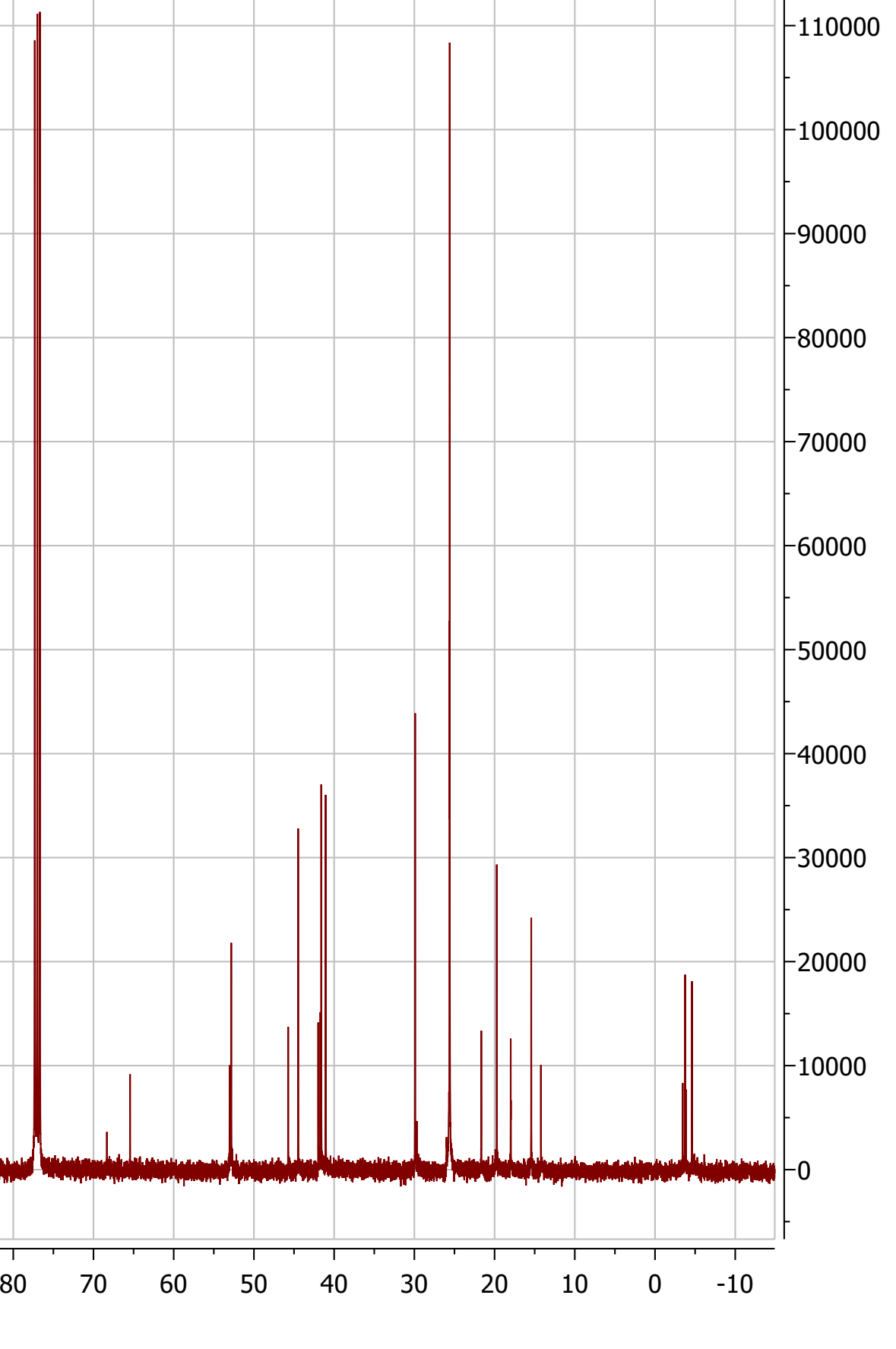




\section{UPC ${ }^{2}$ and HPLC traces}
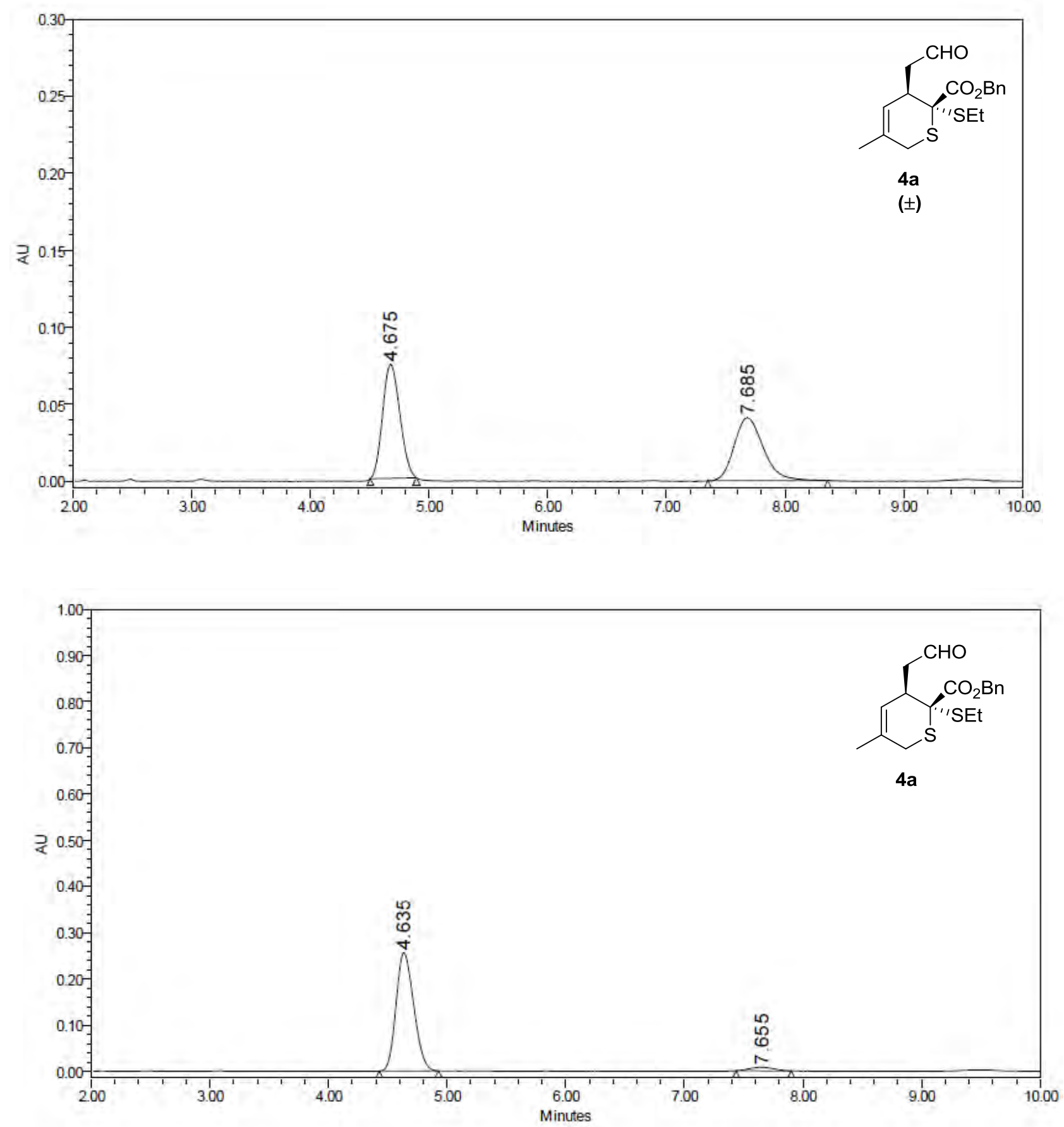

\begin{tabular}{|r|r|r|}
\hline & $\begin{array}{c}\text { Retention Time } \\
\text { (min) }\end{array}$ & \% Area \\
\hline 1 & 4.635 & 96.14 \\
\hline 2 & 7.655 & 3.86 \\
\hline
\end{tabular}



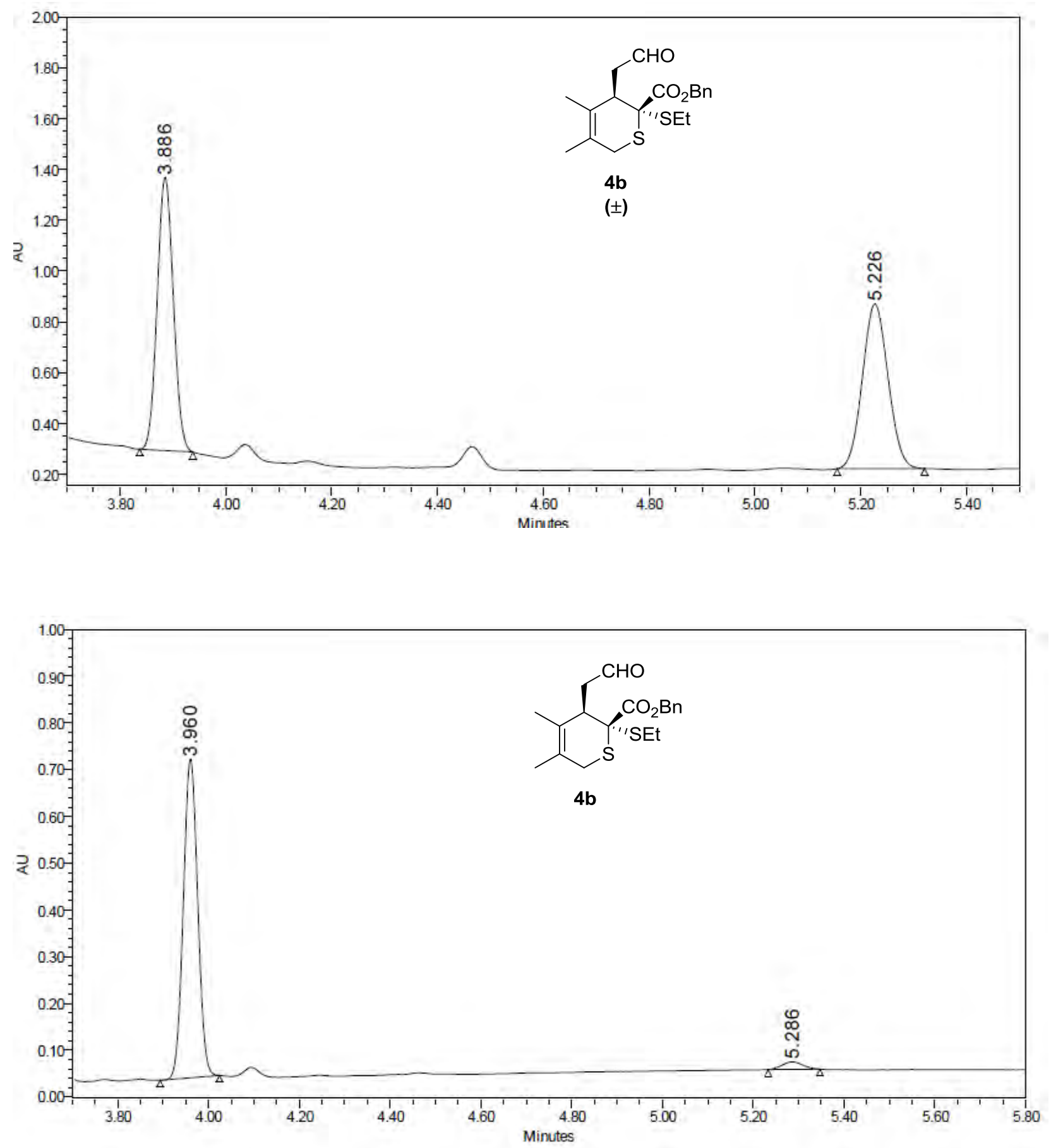

\begin{tabular}{|l|r|r|}
\hline & $\begin{array}{c}\text { Retention Time } \\
\text { (min) }\end{array}$ & \% Area \\
\hline 1 & 3.960 & 96.74 \\
\hline 2 & 5.286 & 3.26 \\
\hline
\end{tabular}



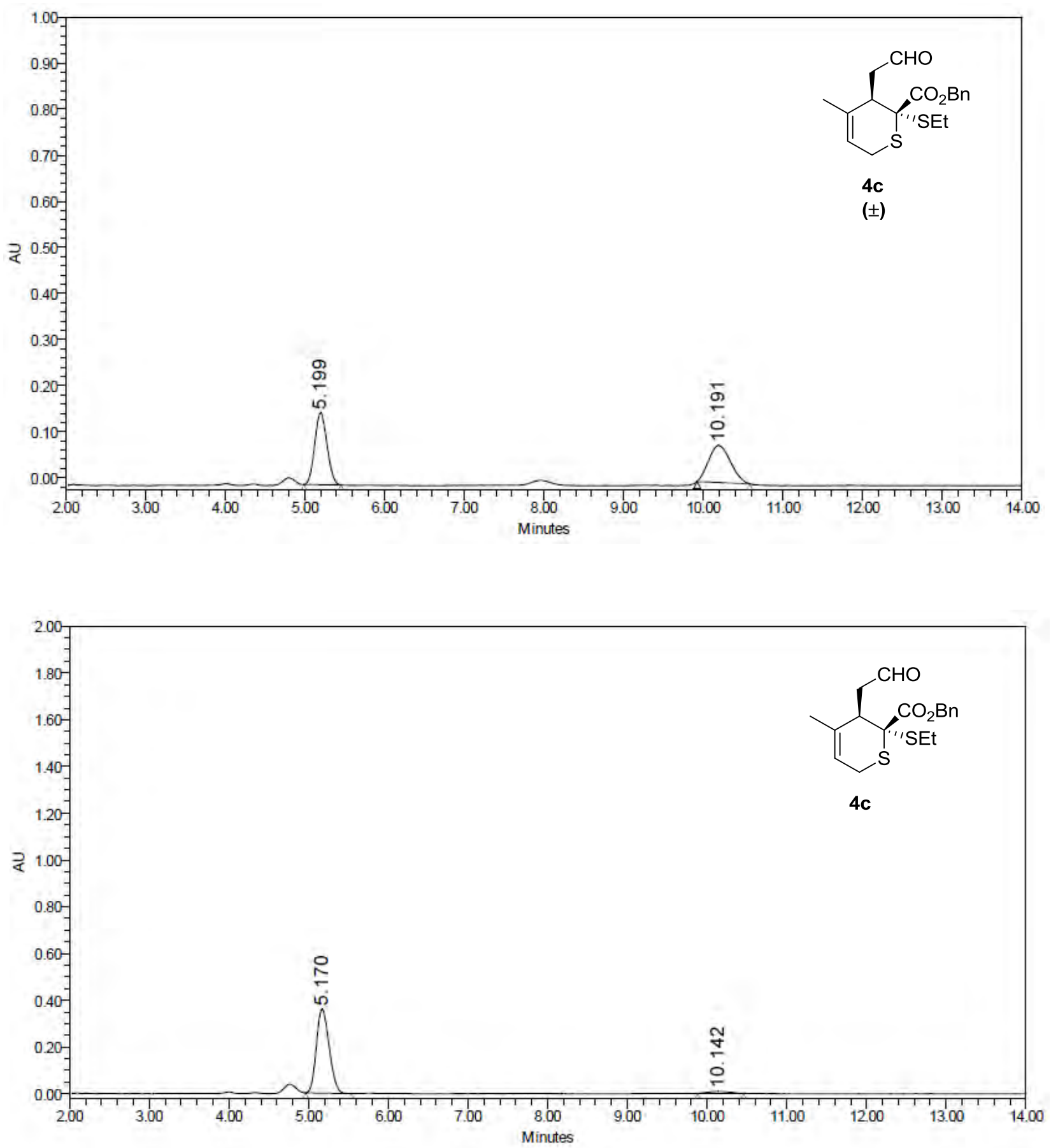

\begin{tabular}{|l|r|r|}
\hline & $\begin{array}{c}\text { Retention Time } \\
\text { (min) }\end{array}$ & $\%$ Area \\
\hline 1 & 5.170 & 96.53 \\
\hline 2 & 10.142 & 3.47 \\
\hline
\end{tabular}



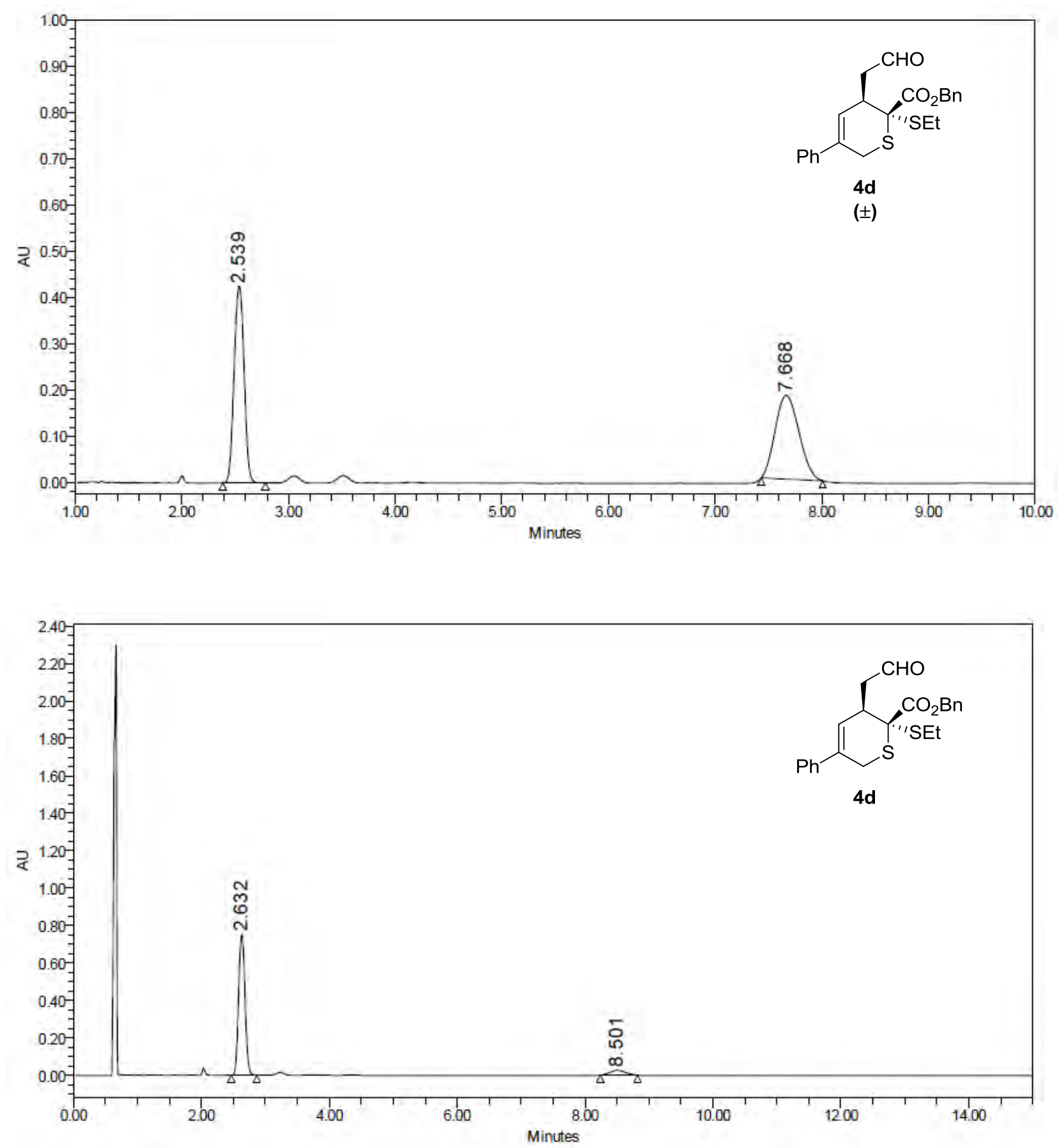

\begin{tabular}{|r|r|r|}
\hline & $\begin{array}{r}\text { Retention Time } \\
\text { (min) }\end{array}$ & $\%$ Area \\
\hline 1 & 2.632 & 92.36 \\
\hline 2 & 8.501 & 7.64 \\
\hline
\end{tabular}



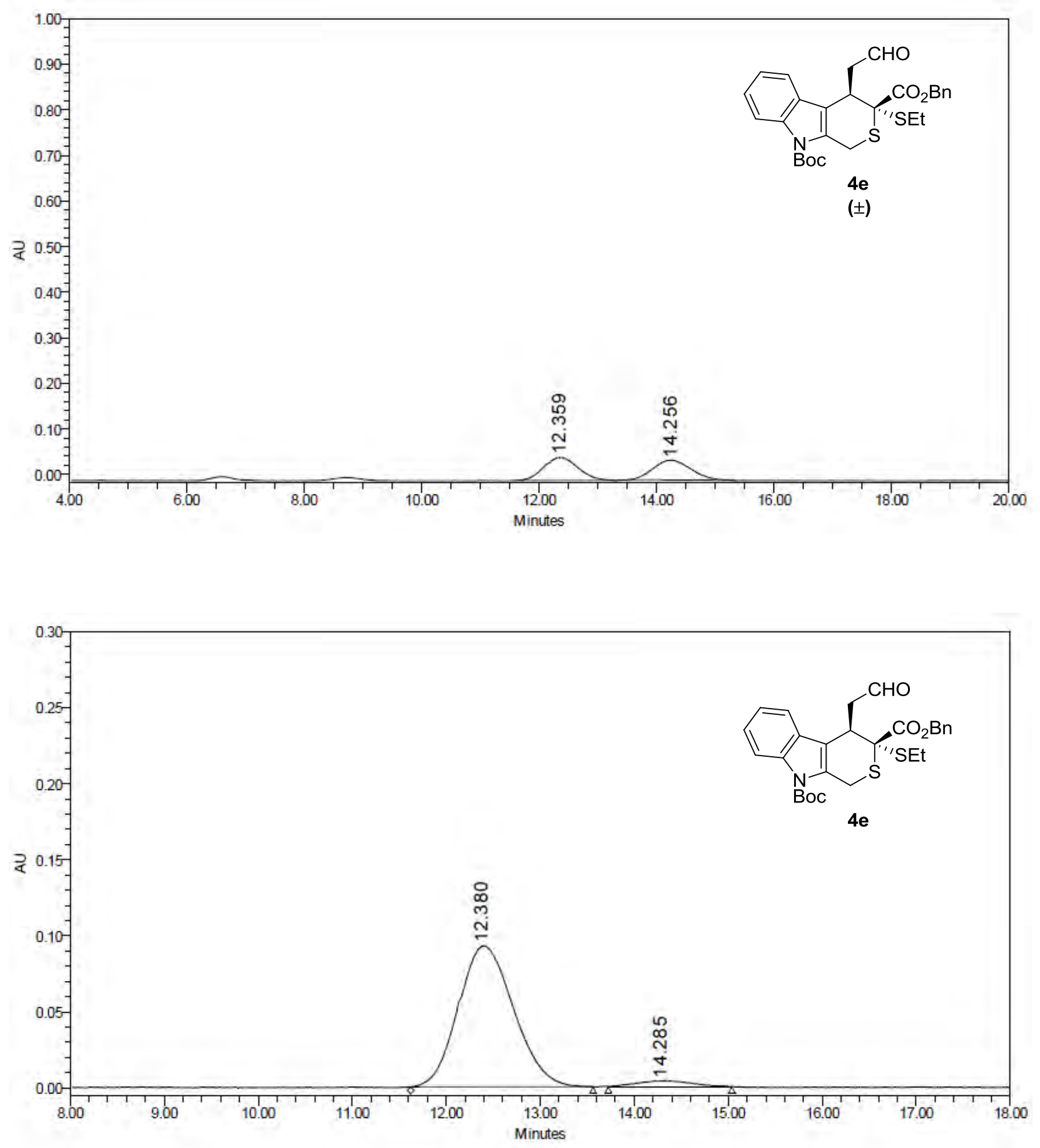

\begin{tabular}{|r|r|r|}
\hline & $\begin{array}{c}\text { Retention Time } \\
\text { (min) }\end{array}$ & $\%$ Area \\
\hline 1 & 12.380 & 96.11 \\
\hline 2 & 14.285 & 3.89 \\
\hline
\end{tabular}



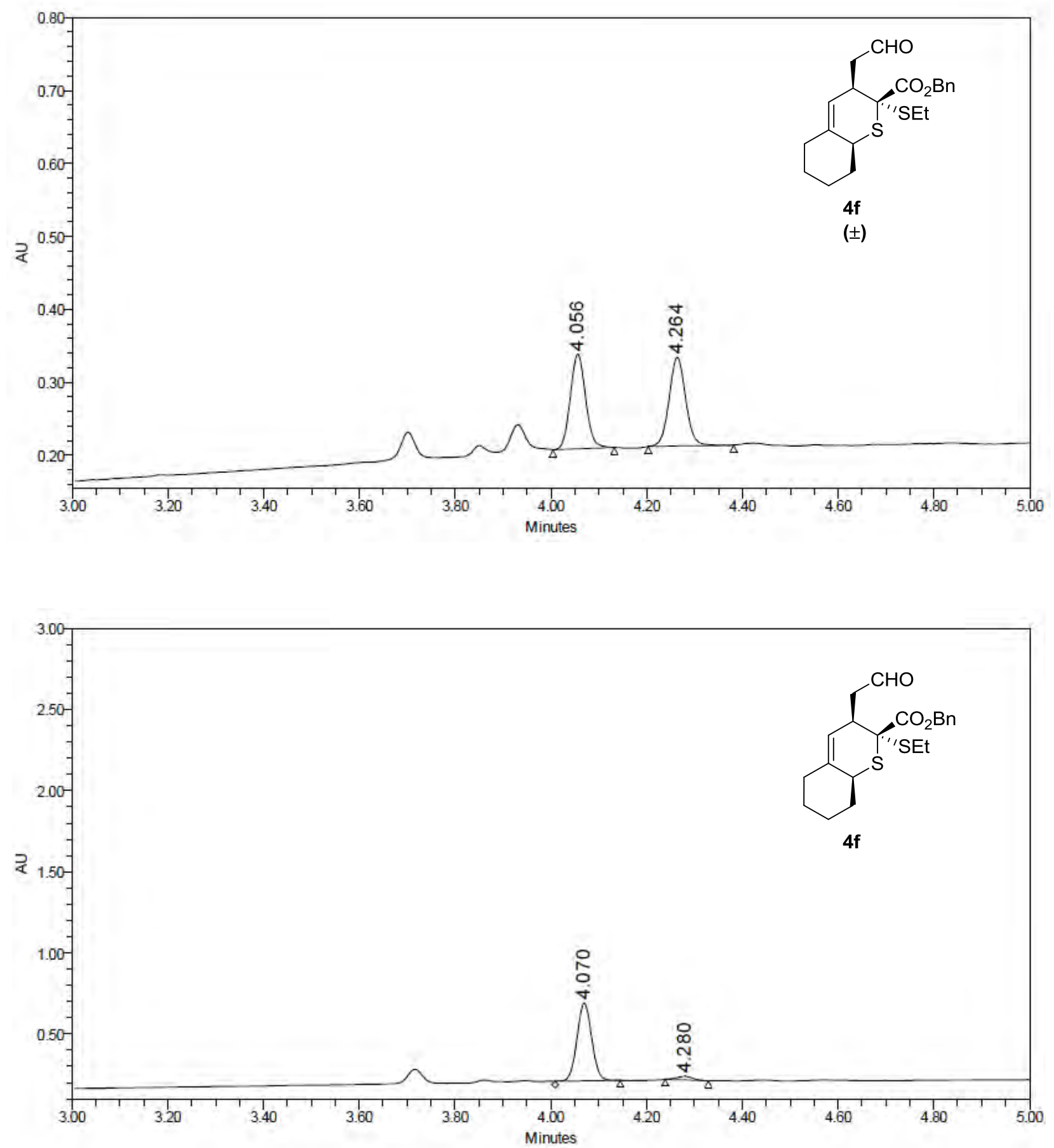

\begin{tabular}{|r|r|r|}
\hline & $\begin{array}{r}\text { Retention Time } \\
\text { (min) }\end{array}$ & $\%$ Area \\
\hline 1 & 4.070 & 95.25 \\
\hline 2 & 4.280 & 4.75 \\
\hline
\end{tabular}



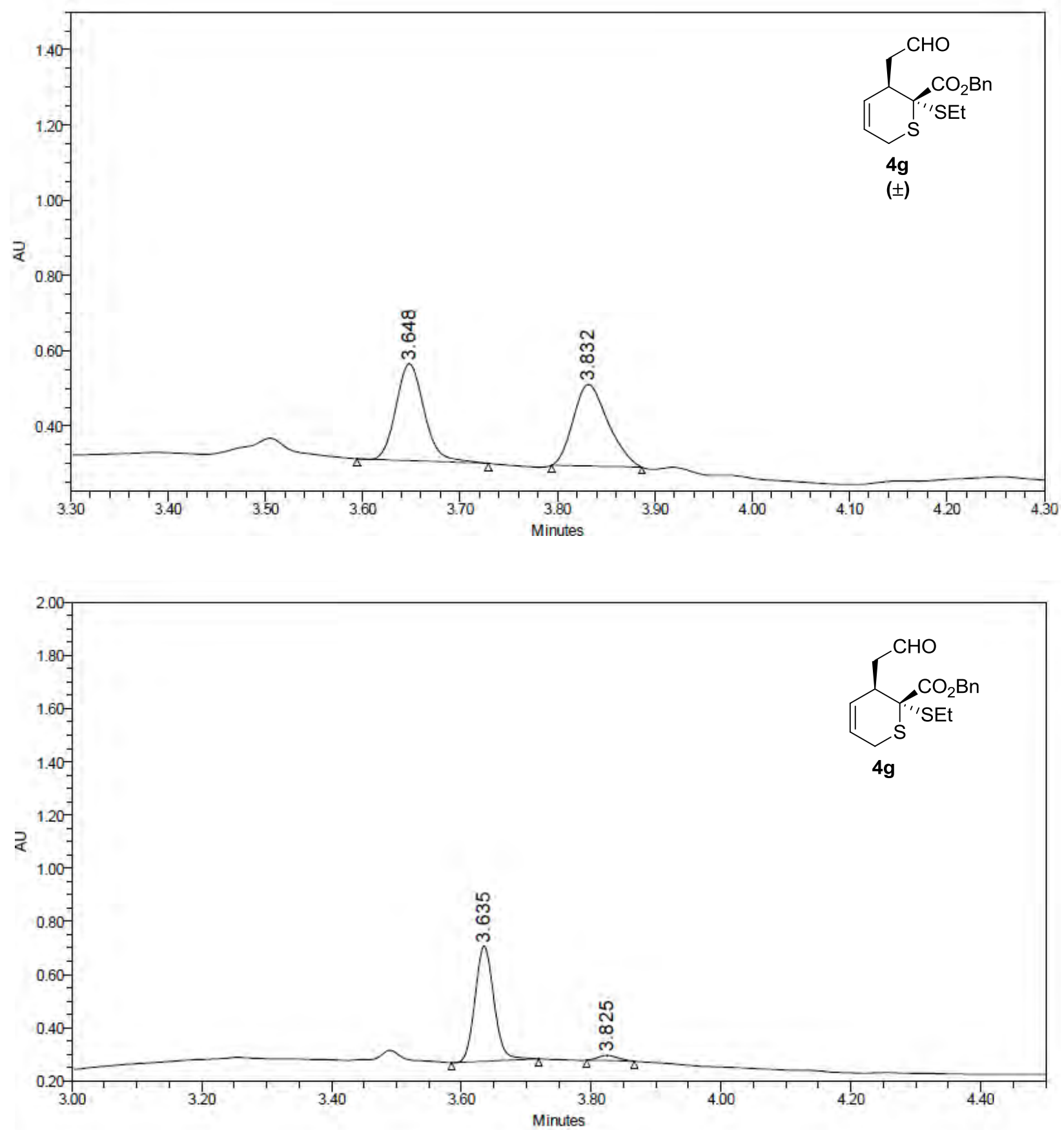

\begin{tabular}{|l|r|r|}
\hline & $\begin{array}{c}\text { Retention Time } \\
\text { (min) }\end{array}$ & $\%$ Area \\
\hline 1 & 3.635 & 95.50 \\
\hline 2 & 3.825 & 4.50 \\
\hline
\end{tabular}



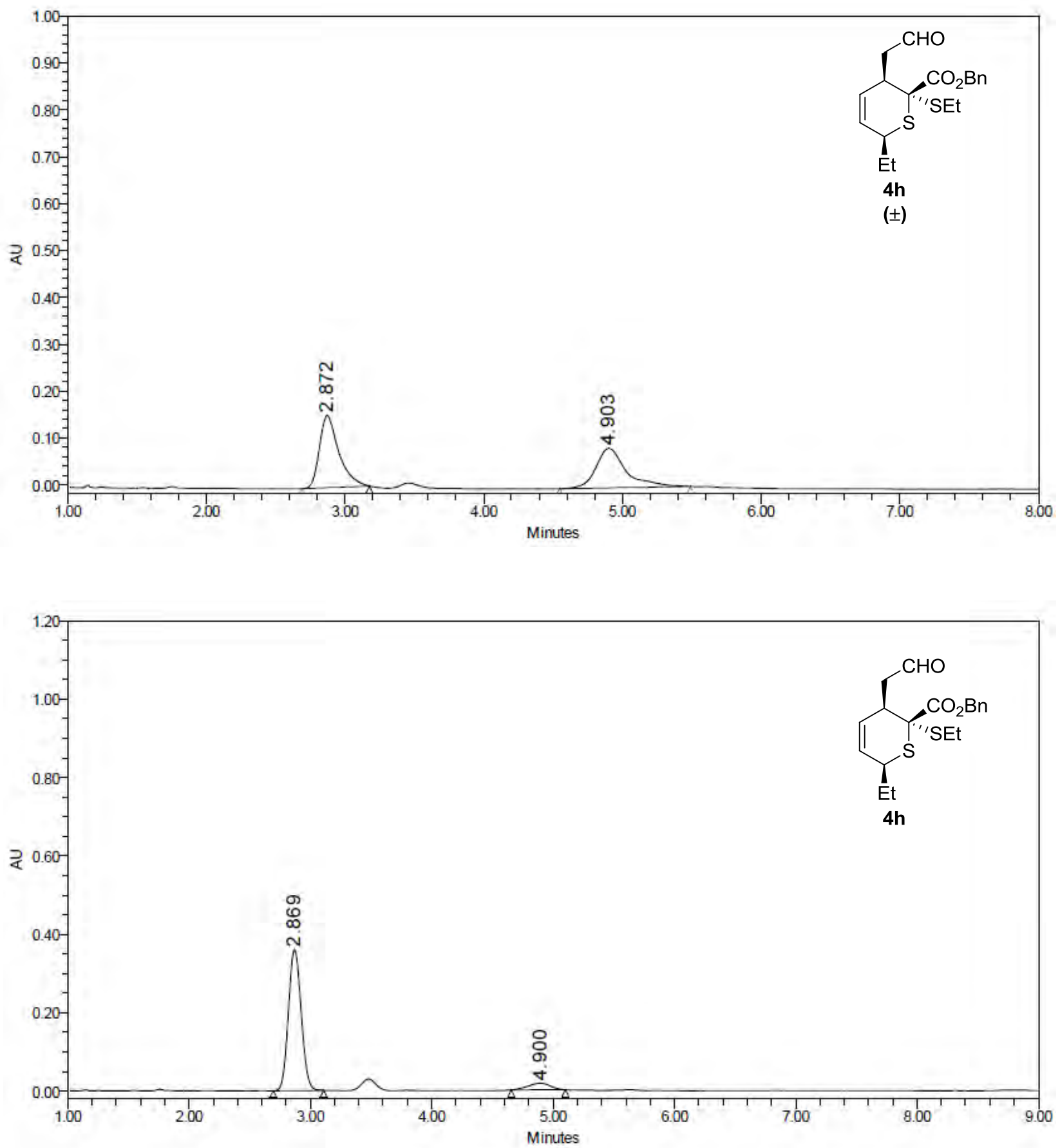

\begin{tabular}{|r|r|r|}
\hline & $\begin{array}{c}\text { Retention Time } \\
\text { (min) }\end{array}$ & $\%$ Area \\
\hline 1 & 2.869 & 92.41 \\
\hline 2 & 4.900 & 7.59 \\
\hline
\end{tabular}



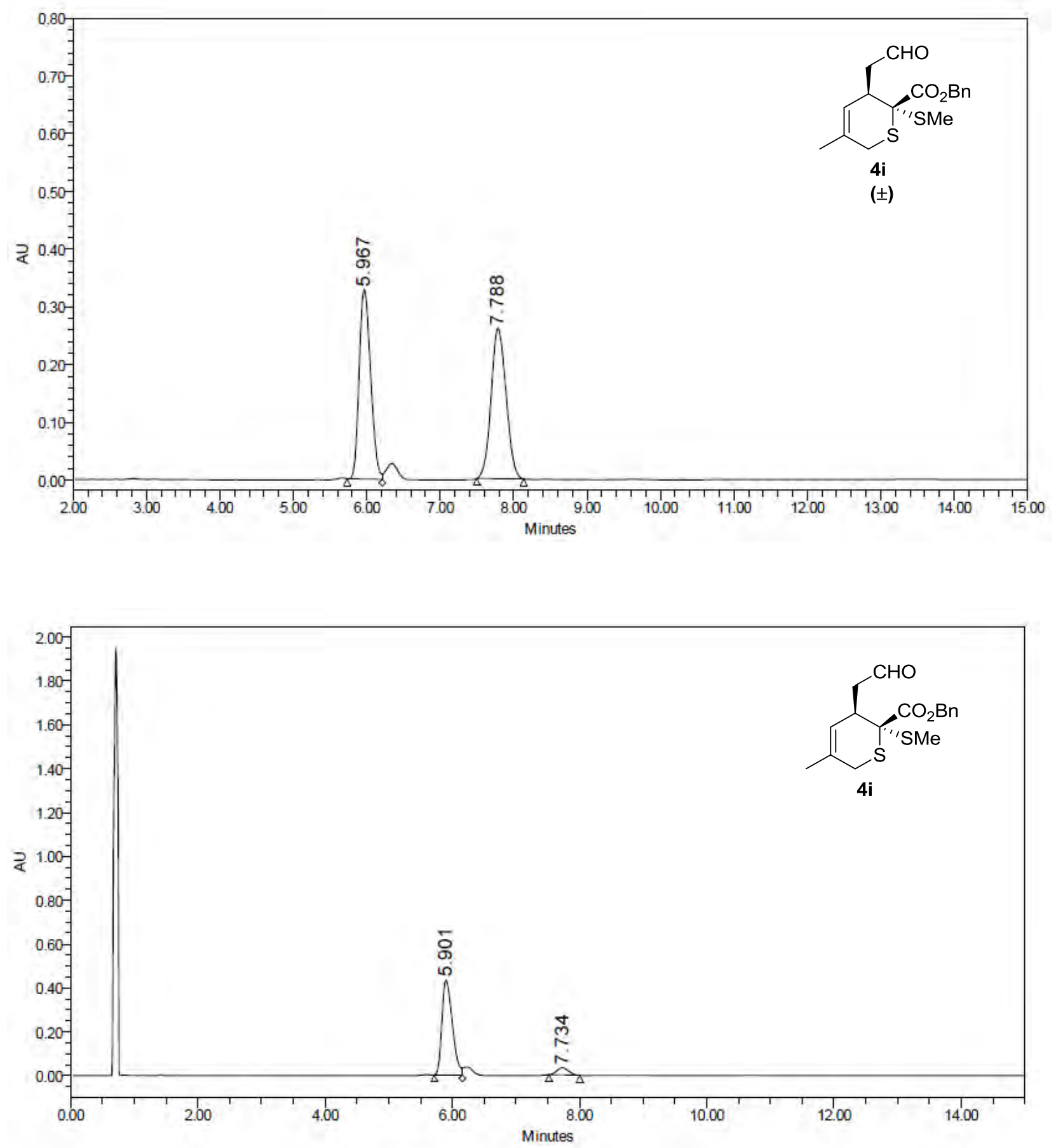

\begin{tabular}{|l|r|r|}
\hline & $\begin{array}{c}\text { Retention Time } \\
(\text { min) }\end{array}$ & \% Area \\
\hline 1 & 5.901 & 92.13 \\
\hline 2 & 7.734 & 7.87 \\
\hline
\end{tabular}



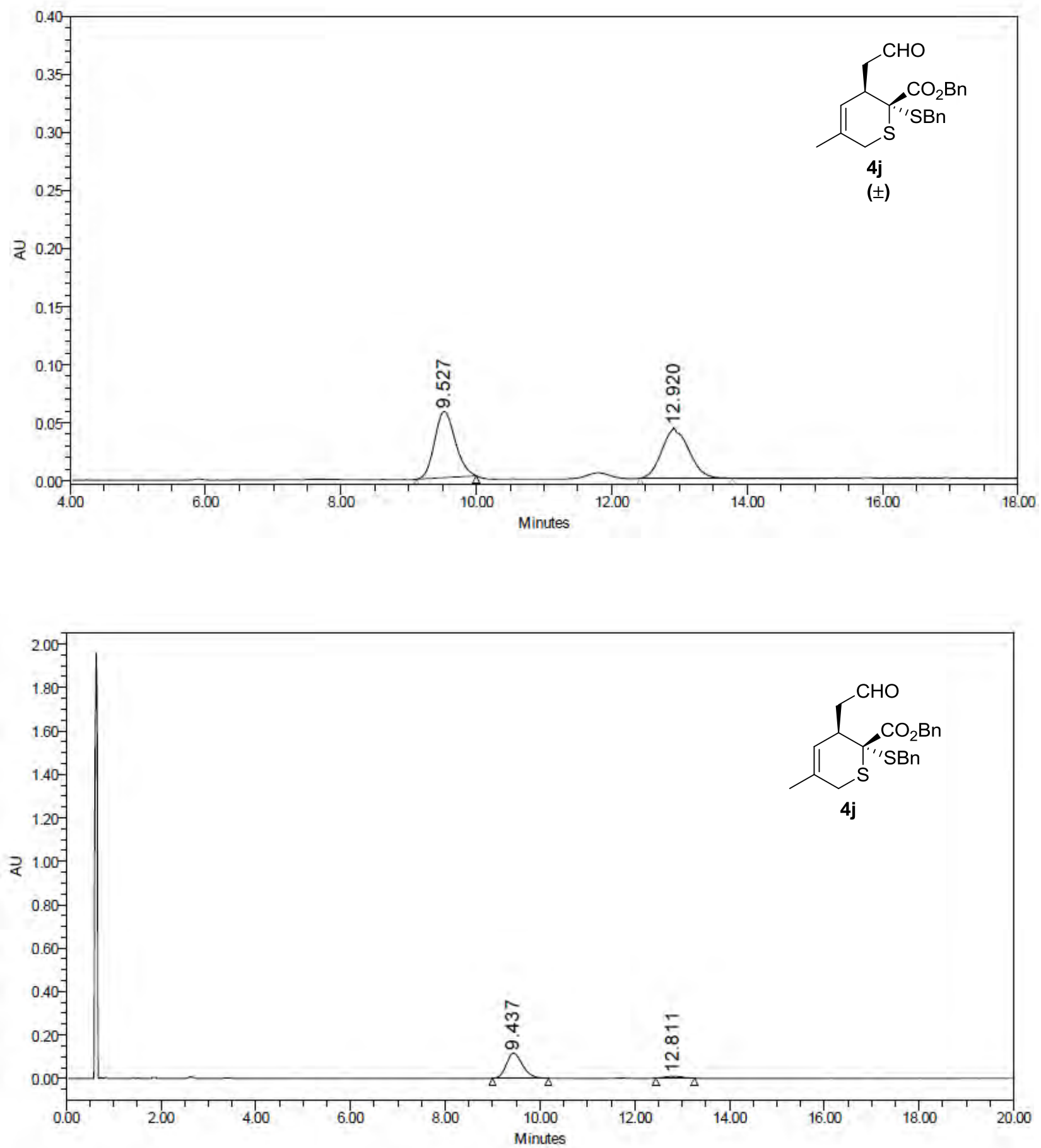

\begin{tabular}{|c|r|r|}
\hline & $\begin{array}{c}\text { Retention Time } \\
\text { (min) }\end{array}$ & $\%$ Area \\
\hline 1 & 9.437 & 92.89 \\
\hline 2 & 12.811 & 7.11 \\
\hline
\end{tabular}



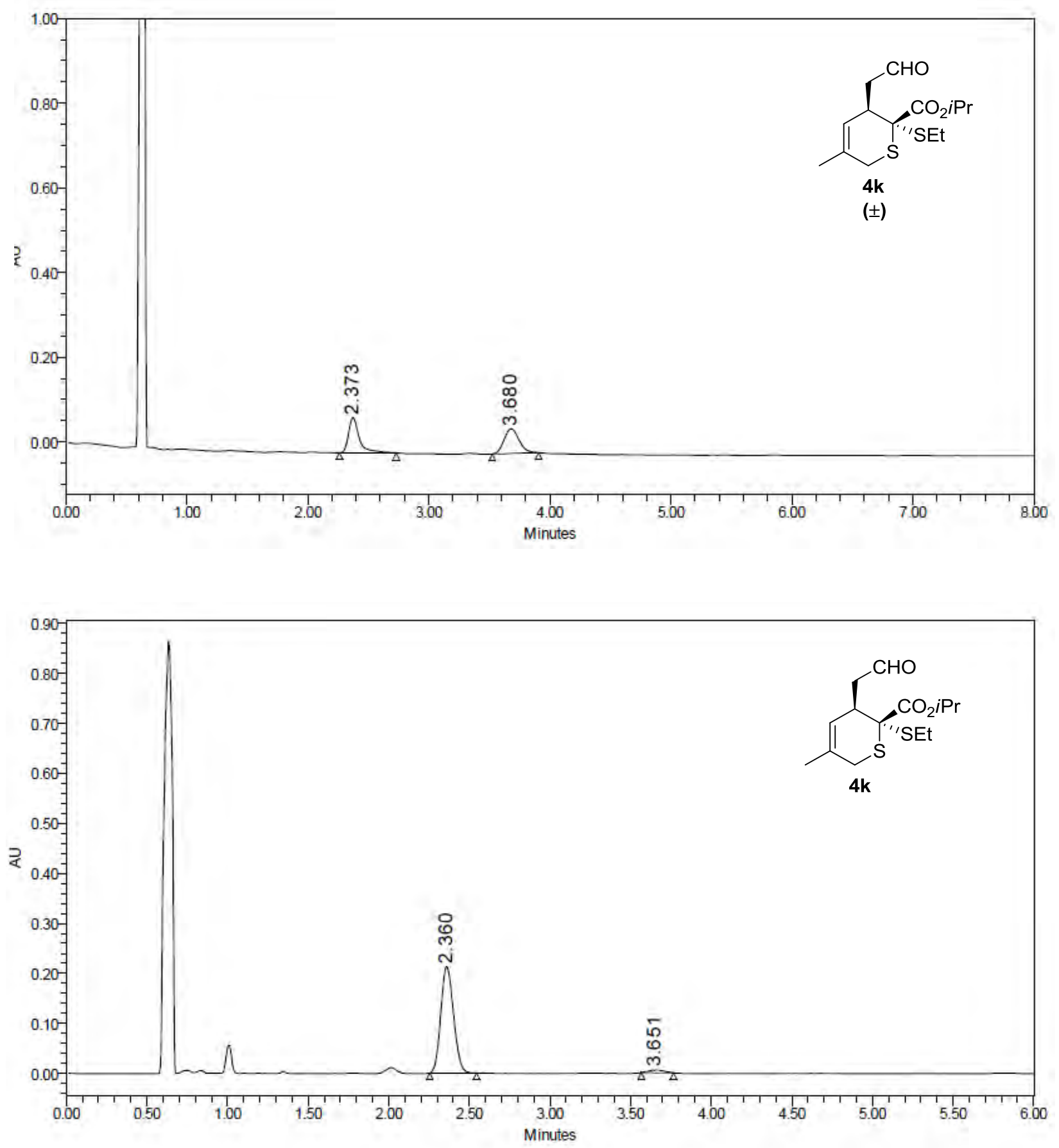

\begin{tabular}{|r|r|r|}
\hline & $\begin{array}{c}\text { Retention Time } \\
\text { (min) }\end{array}$ & $\%$ Area \\
\hline 1 & 2.360 & 97.05 \\
\hline 2 & 3.651 & 2.95 \\
\hline
\end{tabular}



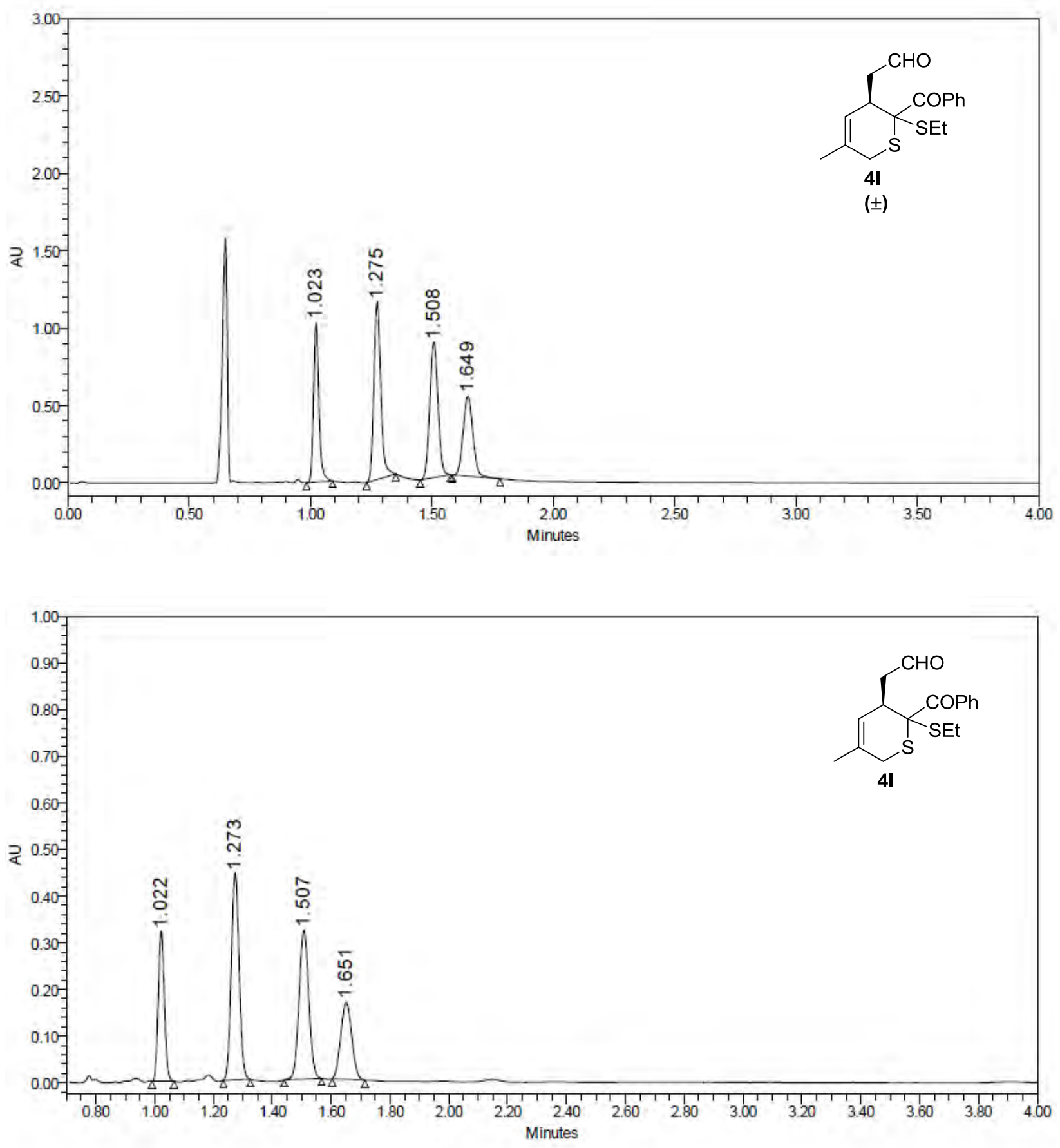

\begin{tabular}{|r|r|r|}
\hline & $\begin{array}{c}\text { Retention Time } \\
\text { (min) }\end{array}$ & $\%$ Area \\
\hline 1 & 1.022 & 18.84 \\
\hline 2 & 1.273 & 33.50 \\
\hline 3 & 1.507 & 30.02 \\
\hline 4 & 1.651 & 17.64 \\
\hline
\end{tabular}



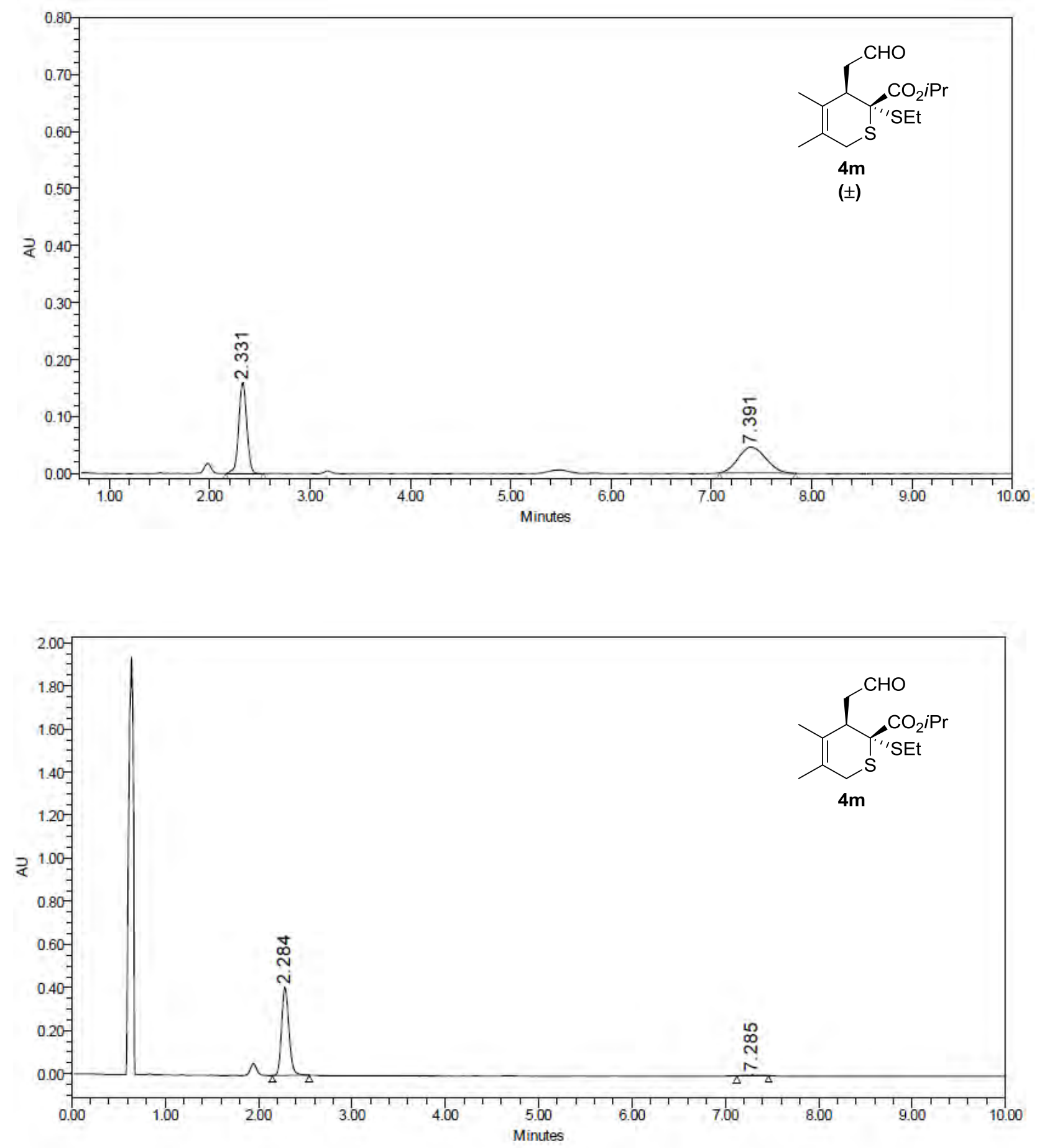

\begin{tabular}{|l|r|r|}
\hline & $\begin{array}{c}\text { Retention Time } \\
\text { (min) }\end{array}$ & $\%$ Area \\
\hline 1 & 2.284 & 98.41 \\
\hline 2 & 7.285 & 1.59 \\
\hline
\end{tabular}



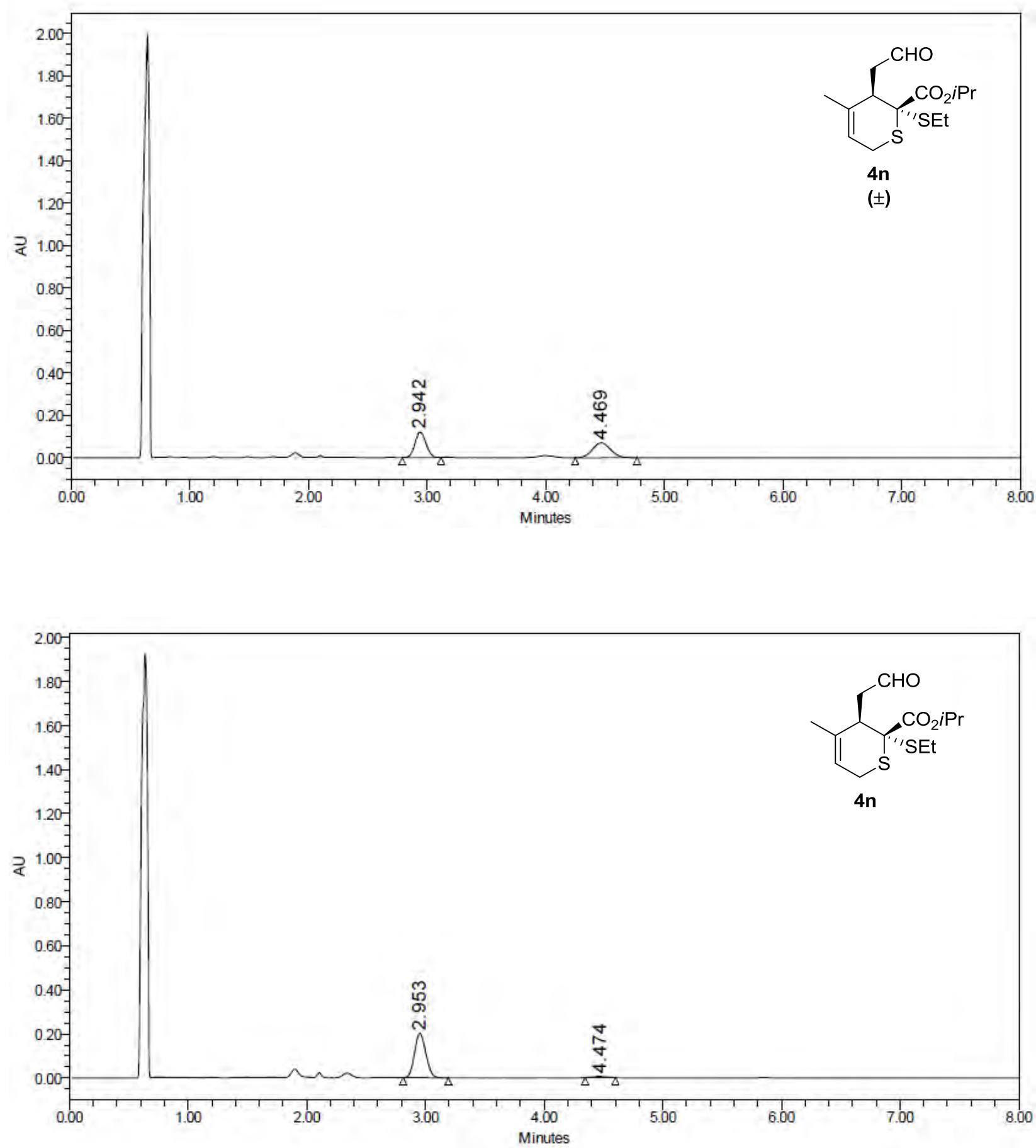

\begin{tabular}{|l|r|r|}
\hline & $\begin{array}{c}\text { Retention Time } \\
\text { (min) }\end{array}$ & $\%$ Area \\
\hline 1 & 2.953 & 96.95 \\
\hline 2 & 4.474 & 3.05 \\
\hline
\end{tabular}



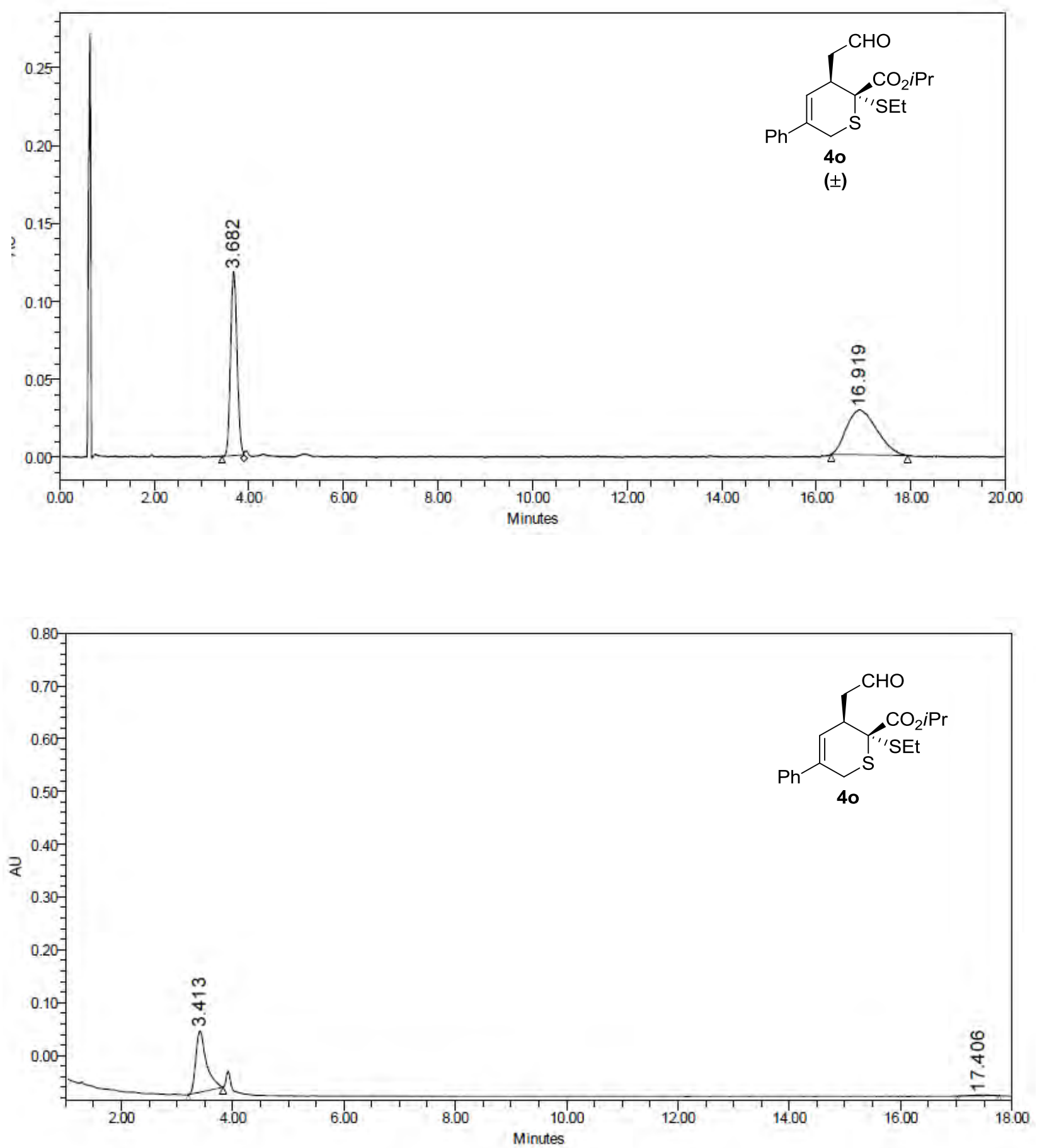

\begin{tabular}{|r|r|r|}
\hline & $\begin{array}{c}\text { Retention Time } \\
\text { (min) }\end{array}$ & $\%$ Area \\
\hline 1 & 17.406 & 2.33 \\
\hline 2 & 3.413 & 97.67 \\
\hline
\end{tabular}



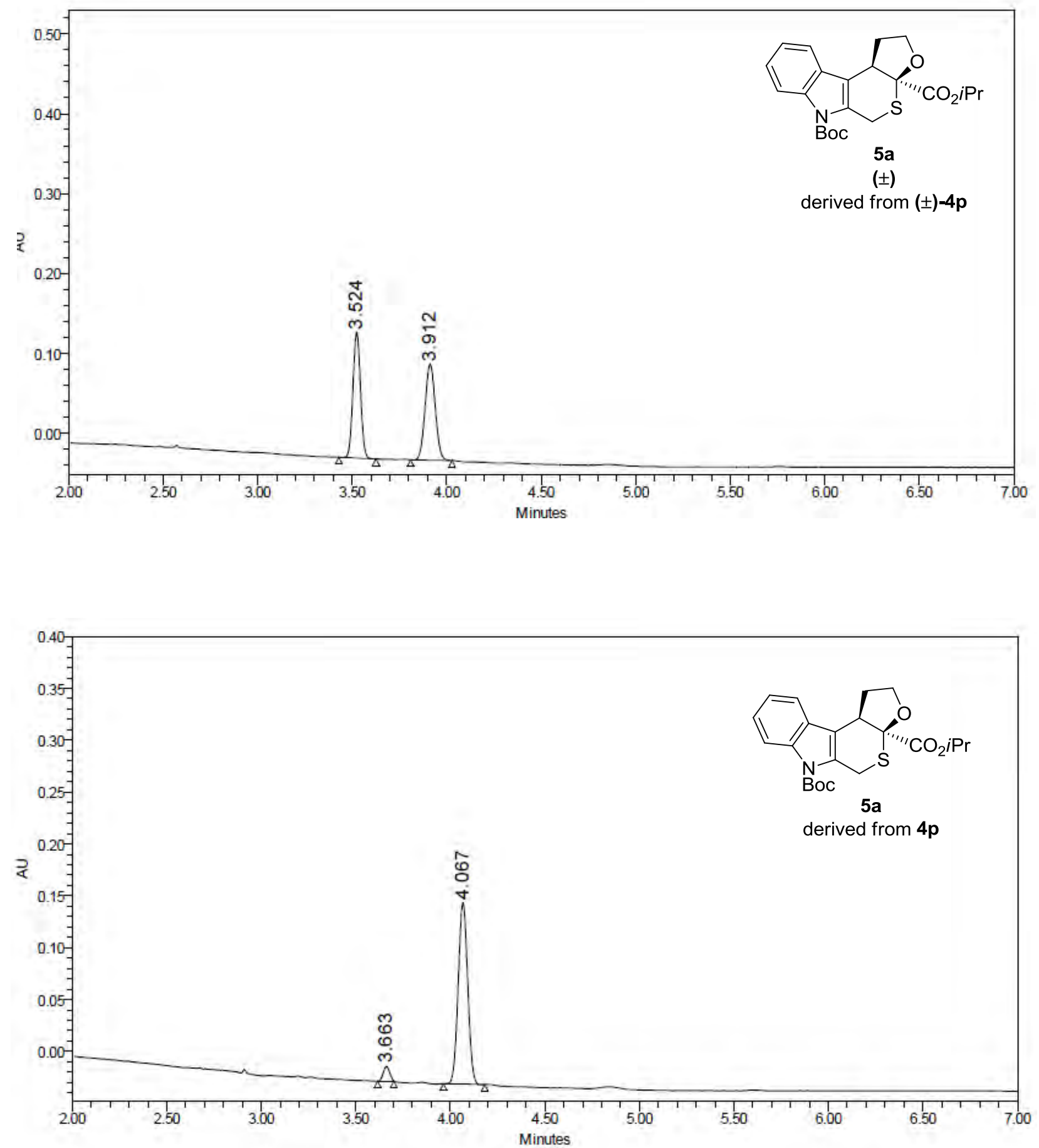

\begin{tabular}{|r|r|r|}
\hline & $\begin{array}{c}\text { Retention Time } \\
\text { (min) }\end{array}$ & \% Area \\
\hline 1 & 3.663 & 5.14 \\
\hline 2 & 4.067 & 94.86 \\
\hline
\end{tabular}



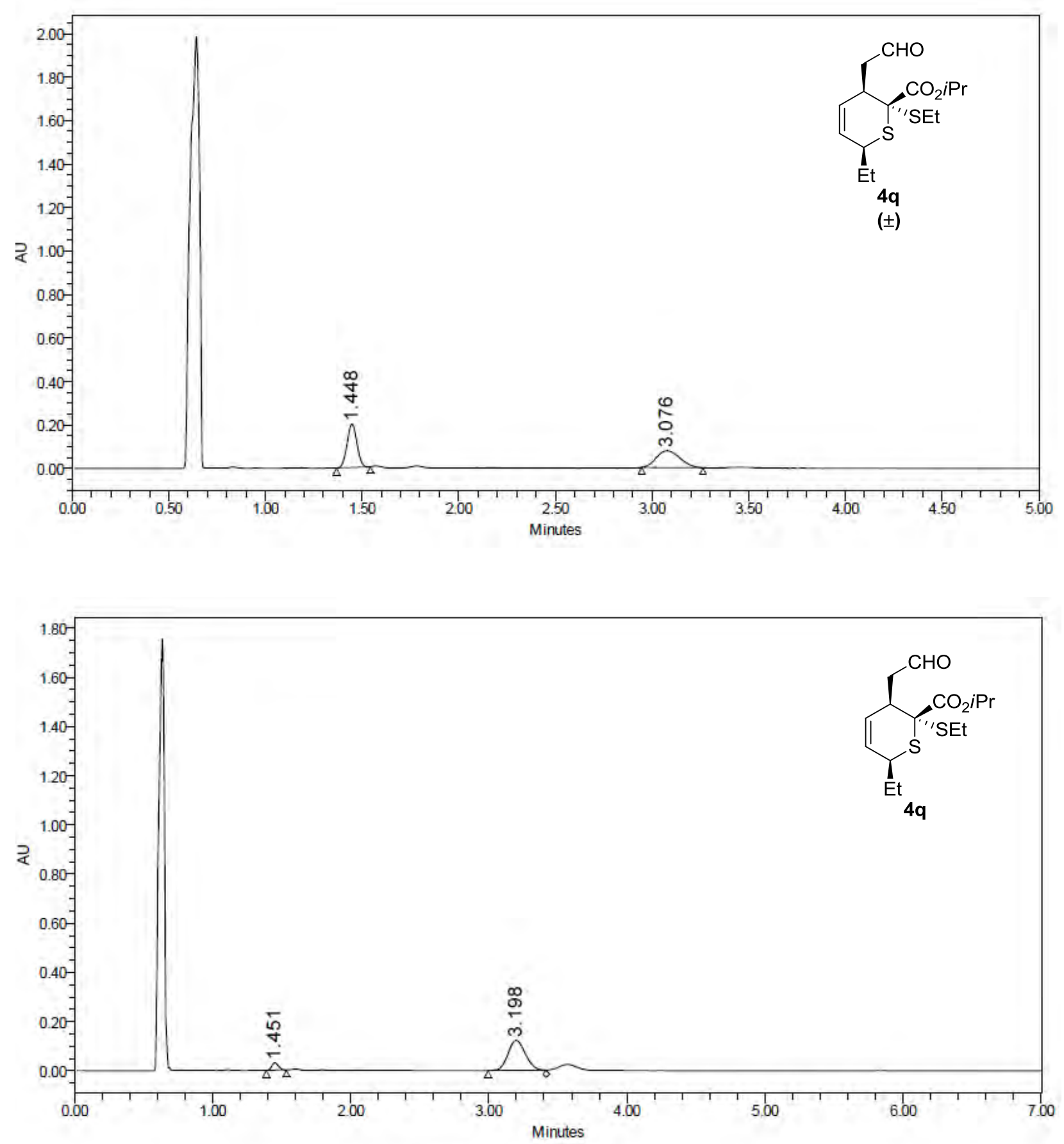

\begin{tabular}{|l|r|r|}
\hline & $\begin{array}{c}\text { Retention Time } \\
\text { (min) }\end{array}$ & $\%$ Area \\
\hline 1 & 1.451 & 8.53 \\
\hline 2 & 3.198 & 91.47 \\
\hline
\end{tabular}



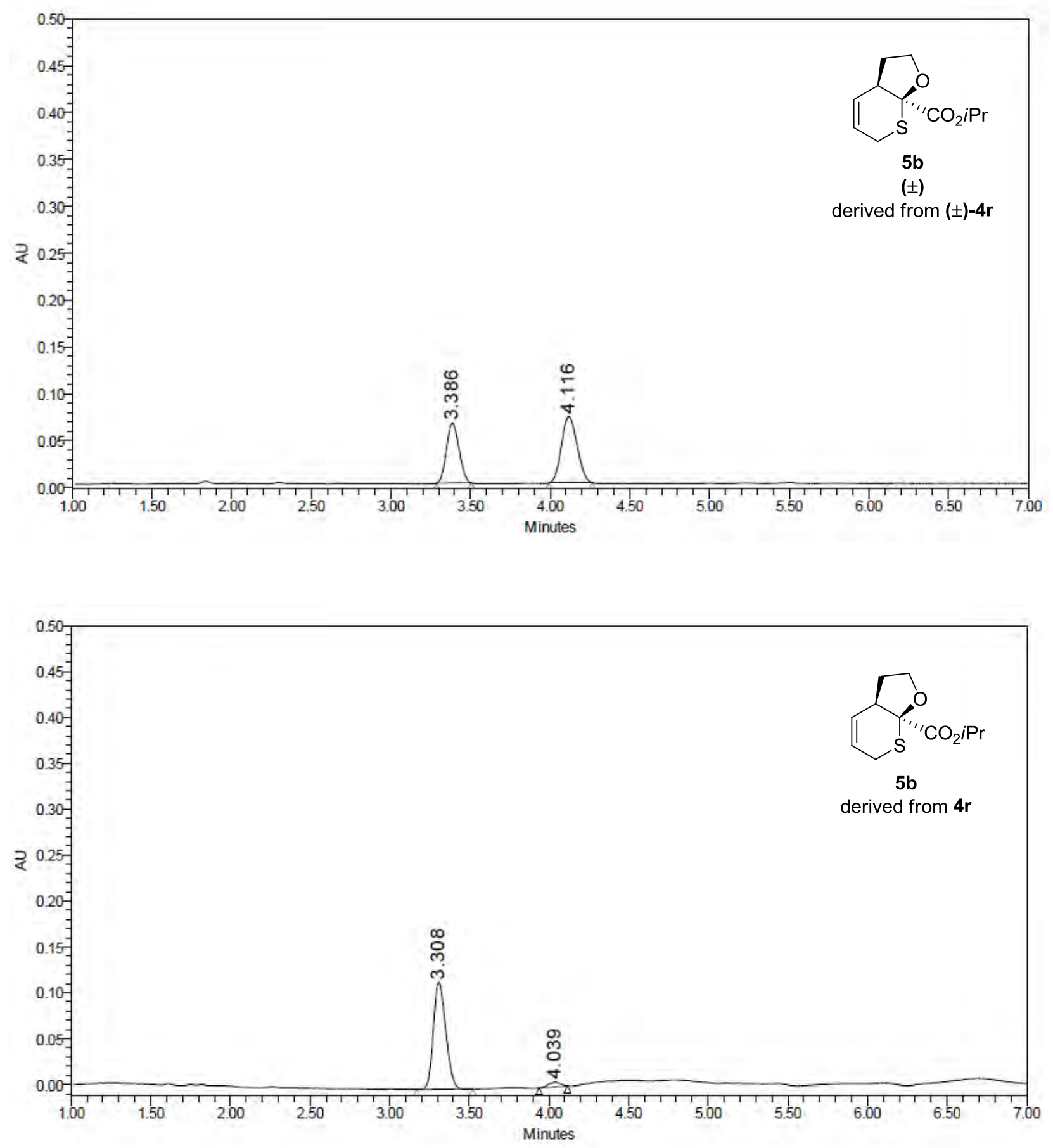

\begin{tabular}{|r|r|r|}
\hline & $\begin{array}{c}\text { Retention Time } \\
\text { (min) }\end{array}$ & $\%$ Area \\
\hline 1 & 3.308 & 95.93 \\
\hline 2 & 4.039 & 4.07 \\
\hline
\end{tabular}

